\title{
Manganese Catalyzed Direct Amidation of Esters with Amines
}

Zhengqiang $\mathrm{Fu}^{\dagger}$, Xinghua Wang ${ }^{\ddagger}$, Sheng Tao ${ }^{\dagger}$, Qingqing $\mathrm{Bu}^{\dagger}$, Donghui Wei ${ }^{\ddagger}, *$ and Ning Liu ${ }^{\dagger} * *$

${ }^{\dagger}$ School of Chemistry and Chemical Engineering, Key Laboratory for Green Processing of Chemical

Engineering of Xinjiang Bingtuan, Shihezi University, North Fourth Road, Shihezi, Xinjiang 832003,

People’s Republic of China, E-mail: ningliu@shzu.edu.cn; ninglau@163.com

${ }^{\ddagger}$ College of Chemistry, Center of Computational Chemistry, Zhengzhou University, Zhengzhou 450001,

People's Republic of China, E-mail: donghuiwei@zzu.edu.cn 


\section{Table of Contents}

1. The Optimization of $t$-BuONa Catalyzed Direct Amidation...............................S2

2. The Effect of Reaction Headspace on Reaction............................. 3

3. Comparision of Reaciton Reactivity for the Amidation....................S4

4. Comparision of Reaciton Reactivity for the Ester, Acid and Amide.............S5

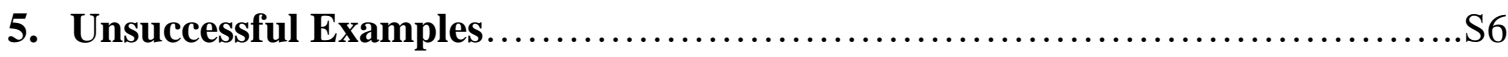

6. Single Crystal Data of Manganese Complexes.................................. $7-9$

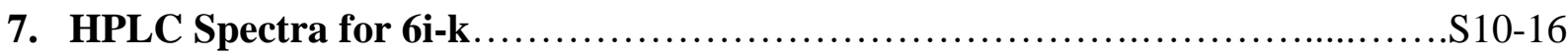

8. UV-vis and IR Spectra for Mechanism................................... $17-18$

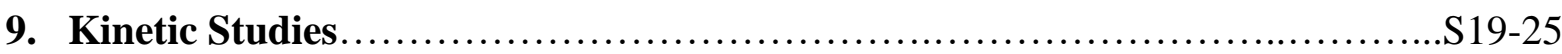

10. NMR Spectra of Manganese Complexes.................................. $26-30$

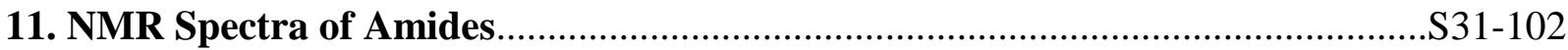

12. The Energies, Geometrical Coordinates, and Frequencies of the Listed Compounds S103-128 


\section{The Optimization of $t$-BuONa Catalyzed Direct Amidation}

Table S1. Direct Amidation of methyl benzoate with benzylamine.<smiles>COC(=O)c1ccccc1</smiles>

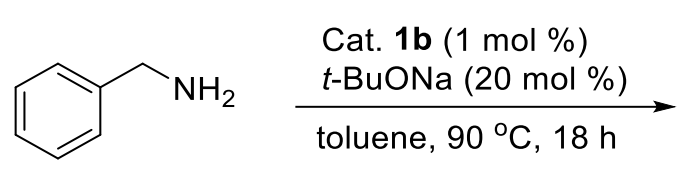<smiles>O=C(NCc1ccccc1)c1ccccc1</smiles>

\begin{tabular}{ccccc}
\hline Entry & Reaction Conditions & Temp $\left({ }^{\circ} \mathrm{C}\right)$ & Solvent & Yield (\%) \\
1 & Cat. 1b $(1 \mathrm{~mol} \%)$ and & 90 & toluene & 88 \\
2 & $t$-BuONa $(20 \mathrm{~mol} \%)$ & 90 & toluene & 10 \\
3 & only $t$-BuONa $(20 \mathrm{~mol} \%)$ & 50 & toluene & 0 \\
4 & only NaOMe $(5 \mathrm{~mol} \%)$ & 50 & 1,4 -dioxane & 0 \\
\hline
\end{tabular}

[a] Reaction conditions: methyl benzoate $(1 \mathrm{mmol})$, benzylamine $(1.2 \mathrm{mmol})$, solvent $(1.5 \mathrm{ml}), 18 \mathrm{~h}$, isolated yields.

Table S2. Direct Amidation of methyl benzoate with morpholine.<smiles>COC(=O)c1ccccc1</smiles><smiles>C1COCCN1</smiles>

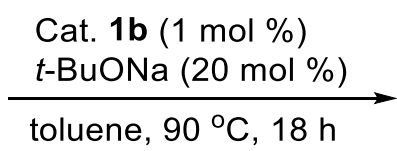<smiles>O=C(c1ccccc1)N1CCOCC1</smiles>

\begin{tabular}{ccclc}
\hline Entry & Reaction Conditions & Temp $\left({ }^{\circ} \mathrm{C}\right)$ & Solvent & Yield $(\%)$ \\
1 & Cat. 1b $(1 \mathrm{~mol} \%)$ and & 90 & toluene & 88 \\
2 & $t$-BuONa $(20$ mol $\%)$ & 90 & toluene & trace \\
3 & only $t$-BuONa $(20$ mol $\%)$ & 90 & DMSO & trace \\
4 & only $t$-BuONa $(20$ mol $\%)$ & 120 & toluene & 0 \\
5 & only $t$-BuOK $(20$ mol\%) & 120 & toluene & 0 \\
6 & only $t$-BuOK $(1$ equiv) & 120 & DMSO & 0 \\
7 & only t-BuOK $(1$ equiv) & 120 & toluene & trace \\
\hline
\end{tabular}

[a] Reaction conditions: methyl benzoate $(1 \mathrm{mmol})$, morpholine $(1.2 \mathrm{mmol})$, solvent $(1.5 \mathrm{ml}), 18 \mathrm{~h}$, isolated yields. 


\section{The effect of the reaction headspace on reaction}<smiles>COC(=O)c1ccccc1</smiles>

$1.2 \mathrm{eq}$

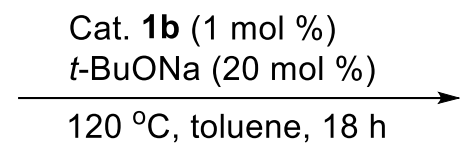

$120^{\circ} \mathrm{C}$, toluene, $18 \mathrm{~h}$
${ }_{1}$

Tall vial : $85 \%$ yield Short vial: $66 \%$ yield

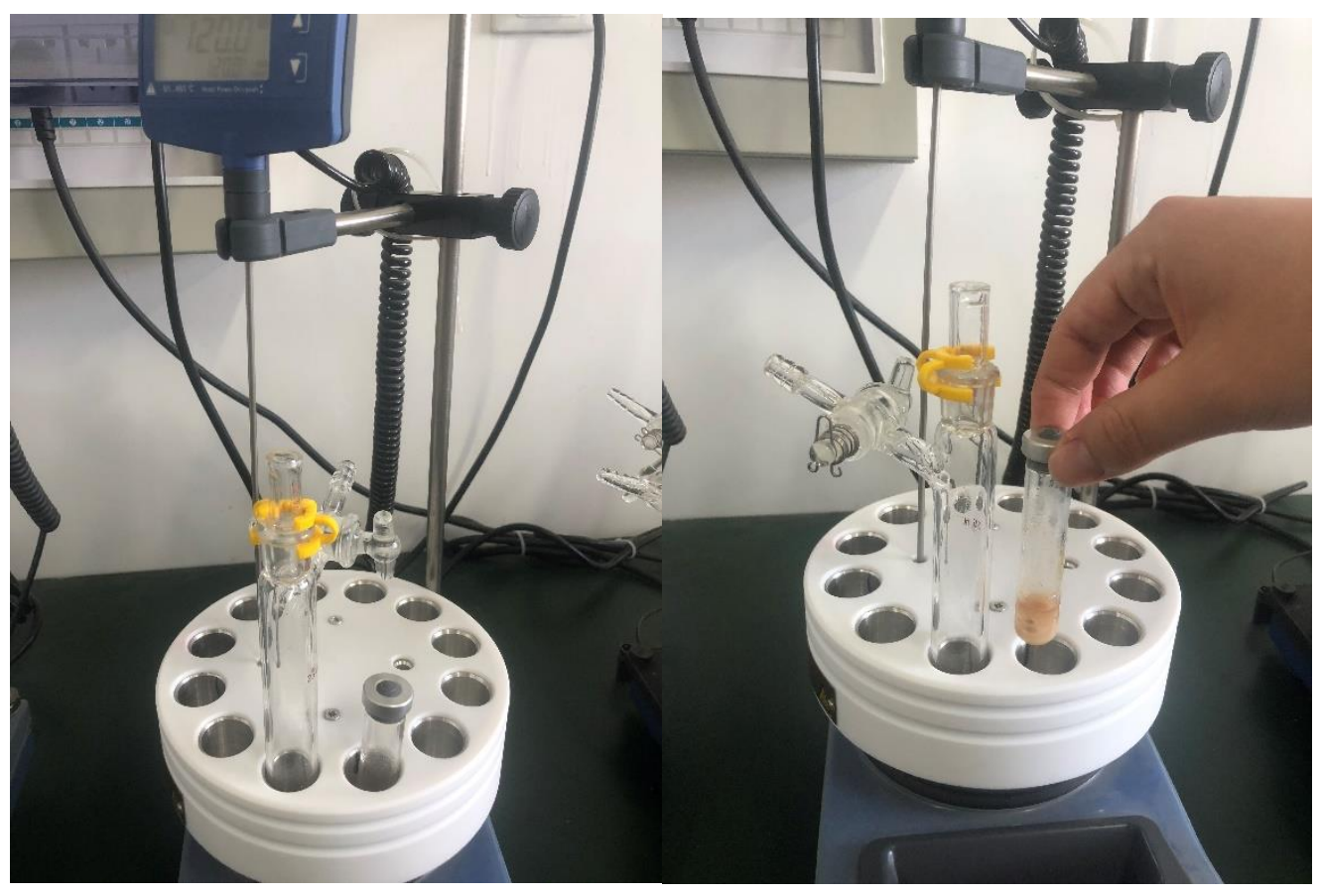

Figure S1. The effect of the reaction headspace on reaction. (The images were photographed by Zhengqiang $\mathrm{Fu}$ ) 


\section{Comparision of reaciton reactivity for the amidation}

Table S3. Comparision of reaciton conditions for different catalytic systems.

\begin{tabular}{|c|c|c|c|c|c|c|c|c|c|}
\hline Entry & Ref. & Esters & Amines & Catalytic system & Base/addtive & $\begin{array}{l}\text { Temp. } \\
\left({ }^{\circ} \mathrm{C}\right)\end{array}$ & $\begin{array}{l}\text { Time } \\
\text { (h) }\end{array}$ & solvent & $\begin{array}{l}\text { Yield } \\
(\%)\end{array}$ \\
\hline 1 & $\operatorname{Garg}^{1}$ & $\begin{array}{l}\text { Methyl } \\
\text { benzoate }\end{array}$ & $\begin{array}{l}N- \\
\text { Methylaniline } \\
\text { ( } 2.0 \text { equiv) }\end{array}$ & $\begin{array}{l}\mathrm{Ni}(\operatorname{cod})_{2}(15 \\
\mathrm{mol} \%) \\
\mathrm{SIPr}(30 \mathrm{~mol} \%)\end{array}$ & $\begin{array}{l}\mathrm{Al}(\mathrm{O} t \mathrm{Bu})_{3} \\
(1.25 \text { equiv })\end{array}$ & 60 & 12 & toluene & 15 \\
\hline 2 & This work & $\begin{array}{l}\text { Methyl } \\
\text { benzoate }\end{array}$ & $\begin{array}{l}N- \\
\text { Methylaniline } \\
\text { ( } 2.0 \text { equiv) }\end{array}$ & $\begin{array}{l}\text { Mn-complex } \\
(1 \mathrm{~mol} \%)\end{array}$ & $\begin{array}{l}t \text {-BuONa } \\
(20 \mathrm{~mol} \%)\end{array}$ & 100 & 18 & toluene & 35 \\
\hline 3 & Newman $^{2}$ & $\begin{array}{l}\text { Phenyl } \\
\text { benzoate }\end{array}$ & $\begin{array}{l}\text { Aniline } \\
\text { (1.2 equiv) }\end{array}$ & $\begin{array}{l}\mathrm{Pd}(\mathrm{IPr})(\text { allyl }) \mathrm{Cl} \\
(3 \mathrm{~mol} \%)\end{array}$ & $\begin{array}{l}\mathrm{K}_{2} \mathrm{CO}_{3}(1.5 \\
\text { equiv), } \mathrm{H}_{2} \mathrm{O} \\
\text { (10 equiv) }\end{array}$ & 110 & 16 & toluene & 91 \\
\hline 4 & This work & $\begin{array}{l}\text { Phenyl } \\
\text { benzoate }\end{array}$ & $\begin{array}{l}\text { Aniline } \\
\text { ( } 1.2 \text { equiv) }\end{array}$ & $\begin{array}{l}\text { Mn-complex } \\
(1 \mathrm{~mol} \%)\end{array}$ & $\begin{array}{l}t \text {-BuONa } \\
(20 \mathrm{~mol} \%)\end{array}$ & 100 & 18 & toluene & 71 \\
\hline 5 & Newman ${ }^{3}$ & $\begin{array}{l}\text { Methyl } \\
\text { benzoate }\end{array}$ & $\begin{array}{l}\text { Aniline } \\
\text { ( } 1.2 \text { equiv) }\end{array}$ & $\begin{array}{l}\mathrm{Ni}(\operatorname{cod})_{2} \\
(10 \mathrm{~mol} \%) \\
\operatorname{IPr}(20 \mathrm{~mol} \%)\end{array}$ & - & 140 & 16 & toluene & 83 \\
\hline 6 & This work & $\begin{array}{l}\text { Methyl } \\
\text { benzoate }\end{array}$ & $\begin{array}{l}\text { Aniline } \\
\text { (1.2 equiv) }\end{array}$ & $\begin{array}{l}\text { Mn-complex } \\
(1 \mathrm{~mol} \%)\end{array}$ & $\begin{array}{l}t \text {-BuONa } \\
(20 \mathrm{~mol} \%)\end{array}$ & 120 & 18 & toluene & 78 \\
\hline 7 & Porco $^{4}$ & $\begin{array}{l}\text { Methyl } \\
\text { cinnamate }\end{array}$ & $\begin{array}{l}\text { Hexylamine } \\
\text { ( } 1.1 \text { equiv) }\end{array}$ & $\begin{array}{l}\mathrm{Zr}(\mathrm{OtBu})_{4} \\
(10 \mathrm{~mol} \%) \\
\text { HOAt }(10 \mathrm{~mol} \%)\end{array}$ & $\begin{array}{l}\text { HOAt } \\
(10 \mathrm{~mol} \%)\end{array}$ & 100 & 12 & toluene & 95 \\
\hline 8 & This work & $\begin{array}{l}\text { Methyl } \\
\text { cinnamate }\end{array}$ & $\begin{array}{l}\text { Hexylamine } \\
\text { ( } 1.2 \text { equiv) }\end{array}$ & $\begin{array}{l}\text { Mn-complex } \\
(1 \mathrm{~mol} \%)\end{array}$ & $\begin{array}{l}t \text {-BuONa } \\
(20 \mathrm{~mol} \%)\end{array}$ & 80 & 18 & toluene & 87 \\
\hline 9 & Mioskowski $^{5}$ & $\begin{array}{l}\text { Methyl } \\
\text { benzoate }\end{array}$ & $\begin{array}{l}\text { Benzylamine } \\
\text { (1.2 equiv) }\end{array}$ & TBD (30 mol\%) & - & 75 & 12 & $\begin{array}{l}\text { solvent- } \\
\text { free }\end{array}$ & 83 \\
\hline 10 & This work & $\begin{array}{l}\text { Methyl } \\
\text { benzoate }\end{array}$ & $\begin{array}{l}\text { Benzylamine } \\
\text { ( } 1.2 \text { equiv) }\end{array}$ & $\begin{array}{l}\text { Mn-complex } \\
(1 \mathrm{~mol} \%)\end{array}$ & $\begin{array}{l}t \text {-BuONa } \\
(20 \mathrm{~mol} \%)\end{array}$ & 70 & 18 & toluene & 84 \\
\hline 11 & Ohshima $^{6}$ & $\begin{array}{l}\text { Methyl 4- } \\
\text { chlorobenzoate }\end{array}$ & $\begin{array}{l}\text { Aniline } \\
\text { ( } 1.3 \text { equiv) }\end{array}$ & $\begin{array}{l}\mathrm{NaOMe}(10 \\
\mathrm{mol} \%)\end{array}$ & $\begin{array}{l}\text { Anhydrous } \\
\text { condition }\end{array}$ & 50 & 120 & toluene & 7 \\
\hline 12 & This work & $\begin{array}{l}\text { Methyl 4- } \\
\text { chlorobenzoate }\end{array}$ & $\begin{array}{l}\text { Aniline } \\
\text { ( } 1.2 \text { equiv) }\end{array}$ & $\begin{array}{l}\text { Mn-complex } \\
(1 \mathrm{~mol} \%)\end{array}$ & $\begin{array}{l}t \text {-BuONa } \\
(20 \mathrm{~mol} \%)\end{array}$ & 80 & 18 & toluene & 43 \\
\hline 13 & Ohshima $^{7}$ & $\begin{array}{l}\text { Methyl } \\
\text { benzoate }\end{array}$ & $\begin{array}{l}\text { Benzylamine } \\
\text { (1.2 equiv) }\end{array}$ & $\begin{array}{l}\mathrm{La}(\mathrm{OTf})_{3} \\
(5 \mathrm{~mol} \%)\end{array}$ & - & 50 & 24 & $\begin{array}{l}\text { solvent- } \\
\text { free }\end{array}$ & 95 \\
\hline 14 & This work & $\begin{array}{l}\text { Methyl } \\
\text { benzoate }\end{array}$ & $\begin{array}{l}\text { Benzylamine } \\
\text { (1.2 equiv) }\end{array}$ & $\begin{array}{l}\text { Mn-complex } \\
(1 \mathrm{~mol} \%)\end{array}$ & $\begin{array}{l}t \text {-BuONa } \\
(20 \mathrm{~mol} \%)\end{array}$ & 50 & 18 & toluene & 64 \\
\hline
\end{tabular}

\section{References}

[1] L. Hie, N. F. Fine Nathel, X. Hong, Y.-F. Yang, K. N. Houk, N. K. Garg, Nickel-Catalyzed Activation of Acyl C-O Bonds of Methyl Esters. Angew. Chem. Int. Ed. 2016, 55, 2810-2814.

[2] T. Ben Halima, J. K. Vandavasi, M. Shkoor, S. G. Newman, A Cross-Coupling Approach to Amide Bond Formation from Esters. ACS Catal. 2017, 7, 2176-2180.

[3] T. Ben Halima, J. Masson-Makdissi, S. G. Newman, Nickel-Catalyzed Amide Bond Formation from Methyl Esters. Angew. Chem. Int. Ed. 2018, 57, 12925-12929.

[4] C. Han, J. P. Lee, E. Lobkovsky, J. A. Porco, Catalytic Ester-Amide Exchange Using Group (IV) Metal Alkoxide-Activator Complexes. J. Am. Chem. Soc. 2005, 127, 10039-10044.

[5] C. Sabot, K. A. Kumar, S. Meunier, C. Mioskowski, A convenient aminolysis of esters catalyzed by 1,5,7-triazabicyclo[4.4.0]dec-5-ene (TBD) under solvent-free conditions. Tetrahedron Lett. 2007, 48, 3863-3866.

[6] T. Ohshima, Y. Hayashi, K. Agura, Y. Fujii, A. Yoshiyama, K. Mashima, Sodium methoxide: a simple but highly efficient catalyst for the direct amidation of esters. Chem. Commun. 2012, 48, 5434-5436.

[7] H. Morimoto, R. Fujiwara, Y. Shimizu, K. Morisaki, T. Ohshima, Lanthanum(III) triflate catalyzed direct amidation of esters. Org. Lett. 2014, 16, 2018-2021. 
4. Comparision of reaciton reactivity for the ester, acid and amide
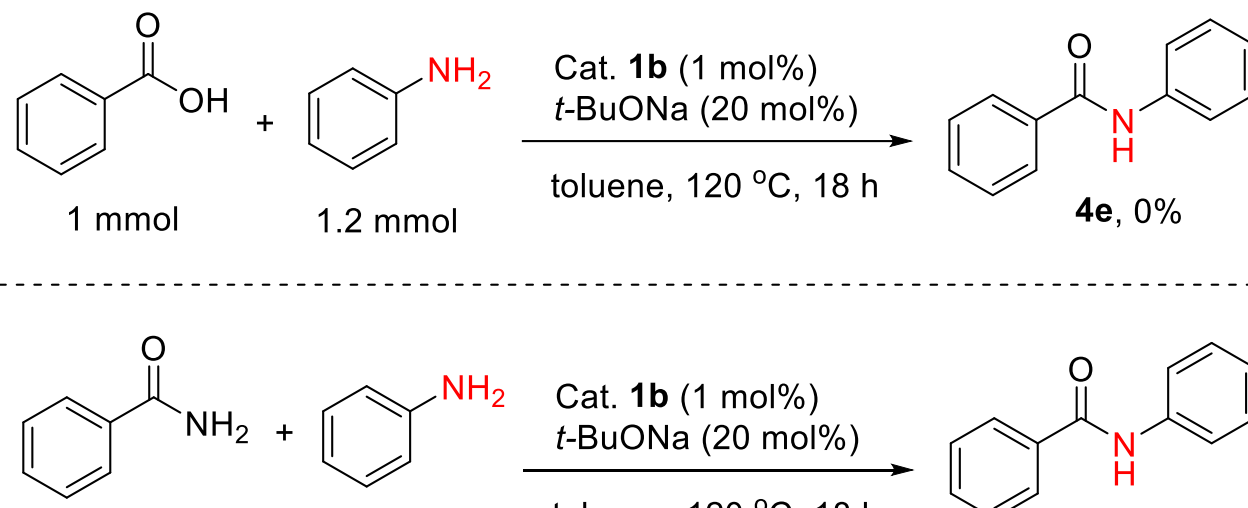

$1 \mathrm{mmol}$

$1.2 \mathrm{mmol}$

Cat. $1 \mathrm{~b}(1 \mathrm{~mol} \%)$

$t$-BuONa (20 mol\%)

toluene, $120^{\circ} \mathrm{C}, 18 \mathrm{~h}$<smiles>O=C(Nc1ccccc1)c1ccccc1</smiles>

4e, $10 \%$<smiles>O=C(O)c1ccccc1</smiles><smiles>COC(=O)c1ccccc1</smiles><smiles>Nc1ccccc1</smiles>

$1.2 \mathrm{mmol}$ $t$-BuONa (20 mol\%)<smiles>NC(=O)c1ccccc1</smiles>

Cat. $1 \mathrm{~b}(1 \mathrm{~mol} \%)$ toluene, $120^{\circ} \mathrm{C}, 18 \mathrm{~h}$<smiles>O=C(Nc1ccccc1)c1ccccc1</smiles>

$4 \mathbf{e}, 36 \%$

Scheme S1. The amidation of esters, acids and amides. 


\section{Unsuccessful Examples}

Our developed Mn-catalytic system was not suitable for 4-vinylaniline, 4-ethynylaniline, and D-valine (Scheme S2).
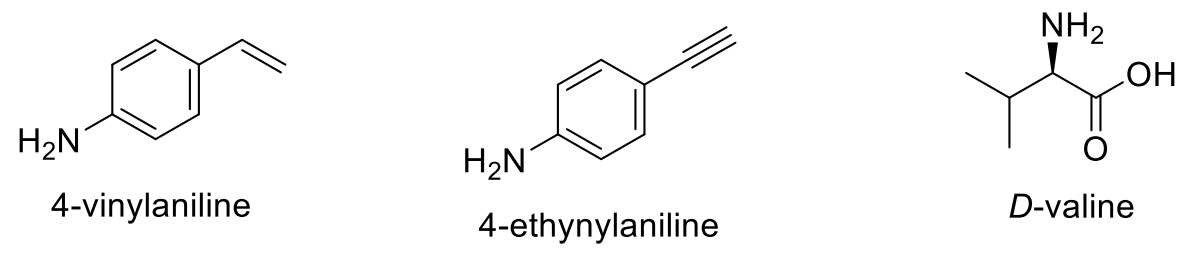

Scheme S2. Unsuccessful Examples. 


\section{Single Crystal data of Manganese Complexes}

Table S4. X-Ray Data of Manganese Complex 1b

\begin{tabular}{|c|c|}
\hline Identification code & $2 \_a$ \\
\hline Empirical formula & C18 H15 I Mn N3 O3 \\
\hline Formula weight & 503.17 \\
\hline Temperature & $173.0 \mathrm{~K}$ \\
\hline Wavelength & $1.54178 \AA$ \\
\hline Crystal system & Monoclinic \\
\hline Space group & $\mathrm{P} 121 / \mathrm{n} 1$ \\
\hline \multirow[t]{3}{*}{ Unit cell dimensions } & $\mathrm{a}=9.0892(2) \AA \quad \alpha=90^{\circ}$. \\
\hline & $\mathrm{b}=13.5502(4) \AA \quad \beta=99.7210(10)^{\circ}$. \\
\hline & $c=15.3227(4) \AA \quad \gamma=90^{\circ}$. \\
\hline Volume & $1860.05(8) \AA^{3}$ \\
\hline $\mathrm{Z}$ & 4 \\
\hline Density (calculated) & $1.797 \mathrm{Mg} / \mathrm{m}^{3}$ \\
\hline Absorption coefficient & $19.001 \mathrm{~mm}^{-1}$ \\
\hline $\mathrm{F}(000)$ & 984 \\
\hline Crystal size & $0.27 \times 0.25 \times 0.22 \mathrm{~mm}^{3}$ \\
\hline Theta range for data collection & 4.384 to $72.423^{\circ}$ \\
\hline Index ranges & $\begin{array}{l}-10<=\mathrm{h}<=11,-13<=\mathrm{k}<=16,- \\
18<=\mathrm{l}<=14\end{array}$ \\
\hline Reflections collected & 11938 \\
\hline Independent reflections & $3640[\mathrm{R}(\mathrm{int})=0.0608]$ \\
\hline Completeness to theta $=67.679^{\circ}$ & $99.3 \%$ \\
\hline Absorption correction & None \\
\hline Refinement method & Full-matrix least-squares on $\mathrm{F}^{2}$ \\
\hline Data / restraints / parameters & $3640 / 0 / 236$ \\
\hline Goodness-of-fit on $\mathrm{F}^{2}$ & 1.044 \\
\hline Final $\mathrm{R}$ indices $[\mathrm{I}>2 \operatorname{sigma}(\mathrm{I})]$ & $\mathrm{R} 1=0.0326, \mathrm{wR} 2=0.0724$ \\
\hline $\mathrm{R}$ indices (all data) & $\mathrm{R} 1=0.0408, \mathrm{wR} 2=0.0765$ \\
\hline Extinction coefficient & $\mathrm{n} / \mathrm{a}$ \\
\hline Largest diff. peak and hole & 0.514 and -0.514 e. $\AA^{-3}$ \\
\hline
\end{tabular}

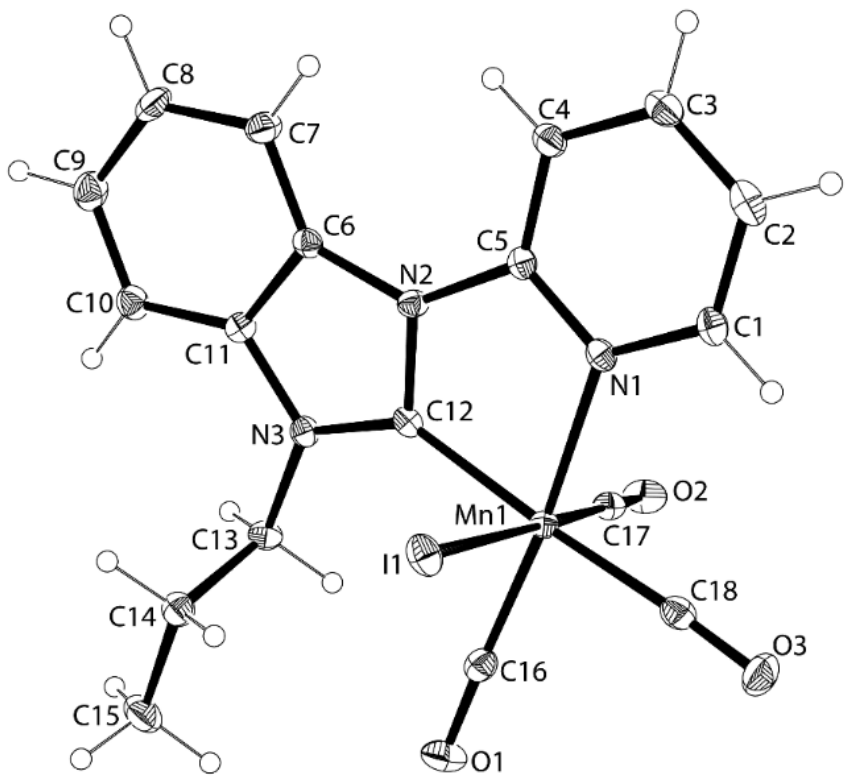

Figure S2. ORTEP of $\mathbf{1 b}$ (at $30 \%$ level) 
Table S5. X-Ray Data of Manganese Complex 1d

\begin{tabular}{|c|c|}
\hline Identification code & 12_a \\
\hline Empirical formula & C18 H15 Br Mn N3 O3 \\
\hline Formula weight & 456.18 \\
\hline Temperature & $173(2) \mathrm{K}$ \\
\hline Wavelength & $0.71073 \AA$ \\
\hline Crystal system & Triclinic \\
\hline Space group & P-1 \\
\hline Unit cell dimensions & $a=9.3052(15) \AA \quad \alpha=77.979(5)^{\circ}$ \\
\hline & $\mathrm{b}=10.212(2) \AA \quad \beta=73.952(5)^{\circ}$ \\
\hline & $\mathrm{c}=10.577(3) \AA \quad \gamma=67.017(7)^{\circ}$ \\
\hline Volume & $883.6(4) \AA^{3}$ \\
\hline $\mathrm{Z}$ & 2 \\
\hline Density (calculated) & $1.715 \mathrm{Mg} / \mathrm{m}^{3}$ \\
\hline Absorption coefficient & $3.031 \mathrm{~mm}^{-1}$ \\
\hline $\mathrm{F}(000)$ & 456 \\
\hline Crystal size & $0.250 \times 0.230 \times 0.220 \mathrm{~mm}^{3}$ \\
\hline Theta range for data collection & 2.435 to $25.363^{\circ}$ \\
\hline Index ranges & $\begin{array}{l}-11<=\mathrm{h}<=10,-12<=\mathrm{k}<=12,- \\
12<=\mathrm{l}<=12\end{array}$ \\
\hline Reflections collected & 14524 \\
\hline Independent reflections & $3238[\mathrm{R}$ (int) $=0.0670]$ \\
\hline Completeness to theta $=25.242^{\circ}$ & $99.9 \%$ \\
\hline Absorption correction & Semi-empirical from equivalents \\
\hline Max. and min. transmission & 0.7452 and 0.5880 \\
\hline Refinement method & Full-matrix least-squares on $\mathrm{F}^{2}$ \\
\hline Data / restraints / parameters & $3238 / 1 / 230$ \\
\hline Goodness-of-fit on $\mathrm{F}^{2}$ & 1.112 \\
\hline Final R indices [I>2sigma(I)] & $\mathrm{R} 1=0.0380, \mathrm{wR} 2=0.1025$ \\
\hline $\mathrm{R}$ indices (all data) & $\mathrm{R} 1=0.0463, \mathrm{wR} 2=0.1062$ \\
\hline Extinction coefficient & $\mathrm{n} / \mathrm{a}$ \\
\hline Largest diff. peak and hole & 1.7260 .917 and -0.417 e. $\AA^{-3}$ \\
\hline
\end{tabular}

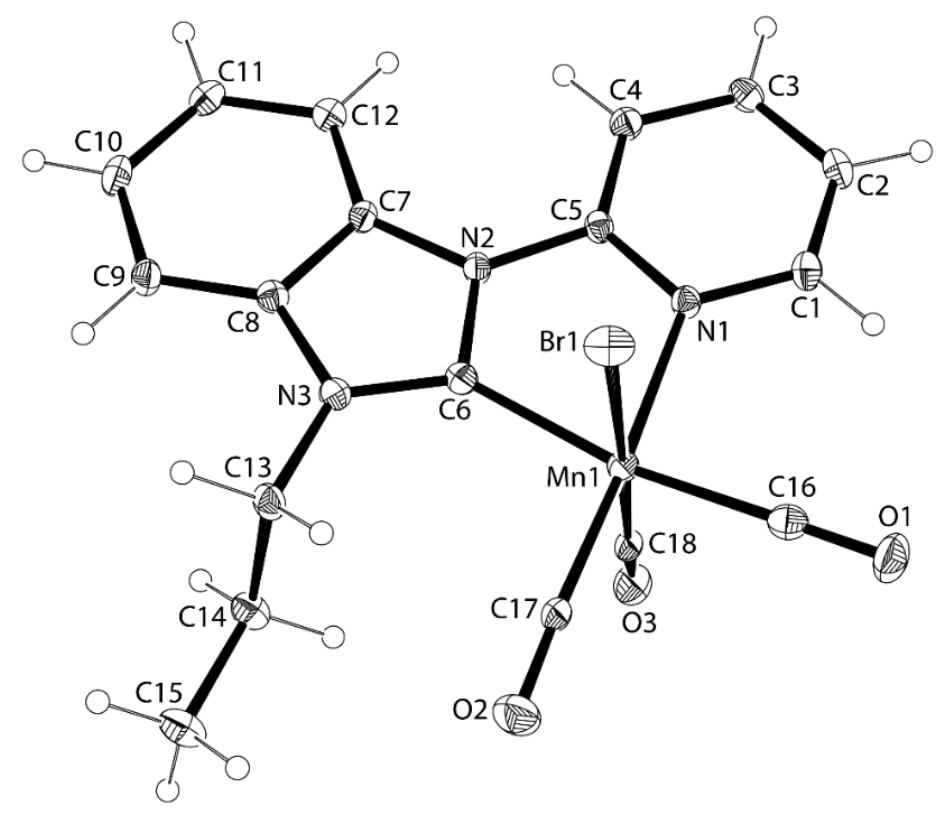

Figure S3. ORTEP of $\mathbf{1 d}$ (at $30 \%$ level) 
Table S6. X-Ray Data of Manganese Complex 1e

\begin{tabular}{|l|l|}
\hline Identification code & TS_Mn1_0m_a \\
\hline Empirical formula & C22 H16 Mn N5 O4 \\
\hline Formula weight & 469.34 \\
\hline Temperature & $150(2) \mathrm{K}$ \\
\hline Wavelength & $1.54178 \AA$ \\
\hline Crystal system & Monoclinic \\
\hline Space group & $\mathrm{P} 21 / \mathrm{n}$ \\
\hline Unit cell dimensions & $\mathrm{a}=7.3270(2) \AA \quad \alpha=90^{\circ}$. \\
\hline & $\mathrm{b}=23.9177(5) \AA \quad \beta=100.0560(10)^{\circ}$. \\
\hline & $\mathrm{c}=11.8844(3) \AA \quad \gamma=90^{\circ}$. \\
\hline Volume & $2050.69(9) \AA^{3}$ \\
\hline Z & 4 \\
\hline Density (calculated) & $1.520 \mathrm{Mg} / \mathrm{m}^{3}$ \\
\hline Absorption coefficient & $5.592 \mathrm{~mm}{ }^{-1}$ \\
\hline F(000) & 960 \\
\hline Crystal size & $0.220 \mathrm{x} 0.200 \mathrm{x} 0.180 \mathrm{~mm}^{3}$ \\
\hline Theta range for data collection & 3.696 to $68.492^{\circ}$. \\
\hline Index ranges & $-8<=\mathrm{h}<=8,-17<=\mathrm{k}<=28,-14<=1<=13$ \\
\hline Reflections collected & 12778 \\
\hline Independent reflections & $3754[\mathrm{R}($ int $)=0.0424]$ \\
\hline Completeness to theta $=67.679^{\circ}$ & $99.6 \%$ \\
\hline Absorption correction & Semi-empirical from equivalents \\
\hline Refinement method & Full-matrix least-squares on $\mathrm{F}^{2}$ \\
\hline Data / restraints / parameters & $3754 / 0 / 290$ \\
\hline Goodness-of-fit on F ${ }^{2}$ & 1.094 \\
\hline Final R indices [I $>2 s i g m a(\mathrm{I})]$ & $\mathrm{R} 1=0.0329, \mathrm{wR} 2=0.0849$ \\
\hline R indices (all data) & $\mathrm{R} 1=0.0378, \mathrm{wR} 2=0.0932$ \\
\hline Extinction coefficient & $\mathrm{n} / \mathrm{a}$ \\
\hline Largest diff. peak and hole & 0.283 and $-0.446 \mathrm{e} . \AA^{-3}$ \\
\hline & \\
\hline
\end{tabular}

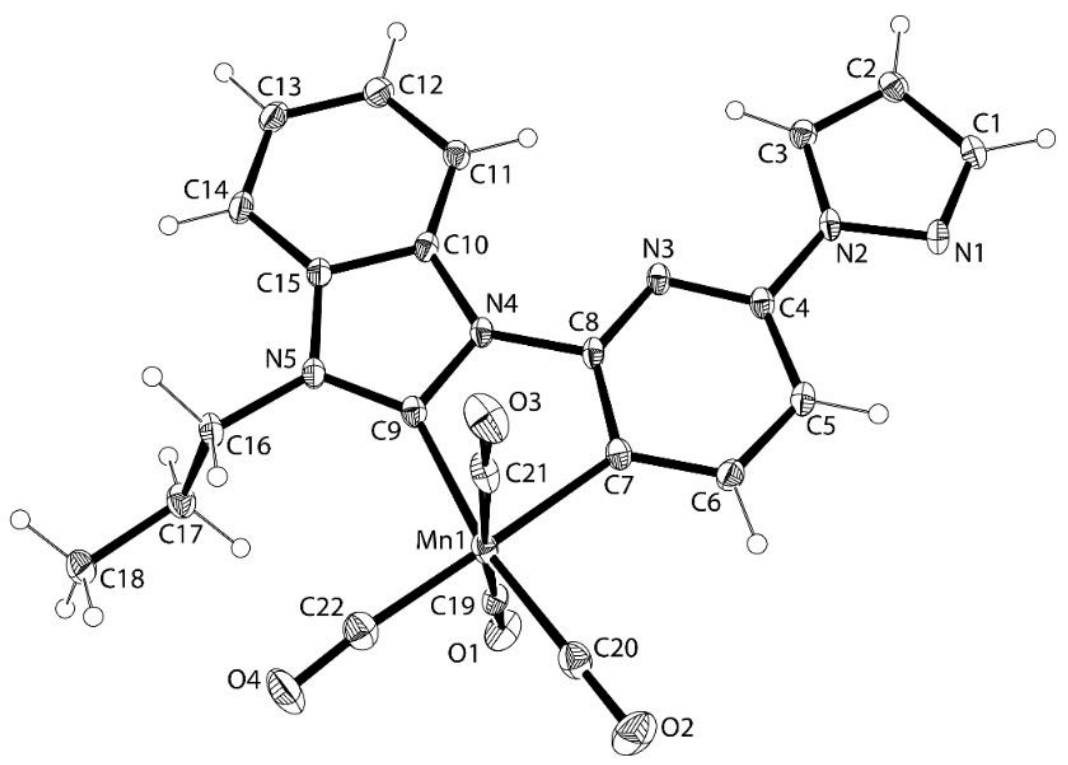

Figure S4. ORTEP of 1e (at 30\% level) 
7. HPLC for $6 \mathbf{i}-\mathrm{k}$

\begin{tabular}{|c|c|c|c|}
\hline Sample Name: & DL-1 & Acquired By: & System \\
\hline Sample Type: & Standard & & \\
\hline Vial: & $1: B, 3$ & Acq. Method Set: & $\begin{array}{l}\mathrm{A}: \mathrm{CO}_{2} \mathrm{~B}: \mathrm{MeOH} \text { (adding } 0.2 \% \\
\text { TFA) } \\
\text { 0-20min: } 28 \%-58 \% \text { MeOH (adding } \\
0.2 \% \text { TFA); } 20.01-25 \mathrm{~min}: 28 \% \\
\mathrm{MeOH} \text { (adding } 0.2 \% \text { TFA) } \\
\text { Column: Chiralcel OD-H 4.6*250nm } \\
\text { 5um }\end{array}$ \\
\hline Injection \#: & 1 & & \\
\hline $\begin{array}{l}\text { Injection } \\
\text { Volume }\end{array}$ & $10.0 \mathrm{ul}$ & Channel Name: & $254.0 \mathrm{~nm}$ \\
\hline $\begin{array}{c}\text { Run Time: } \\
\text { Date acquired: }\end{array}$ & $\begin{array}{c}\text { 25.0 Minutes } \\
\text { 2020/6/22 18:31:25 }\end{array}$ & & \\
\hline $\begin{array}{c}\text { Date } \\
\text { Processed: }\end{array}$ & 2020/6/23 09:16:24 & & \\
\hline
\end{tabular}

Auto-Scaled Chromatogram

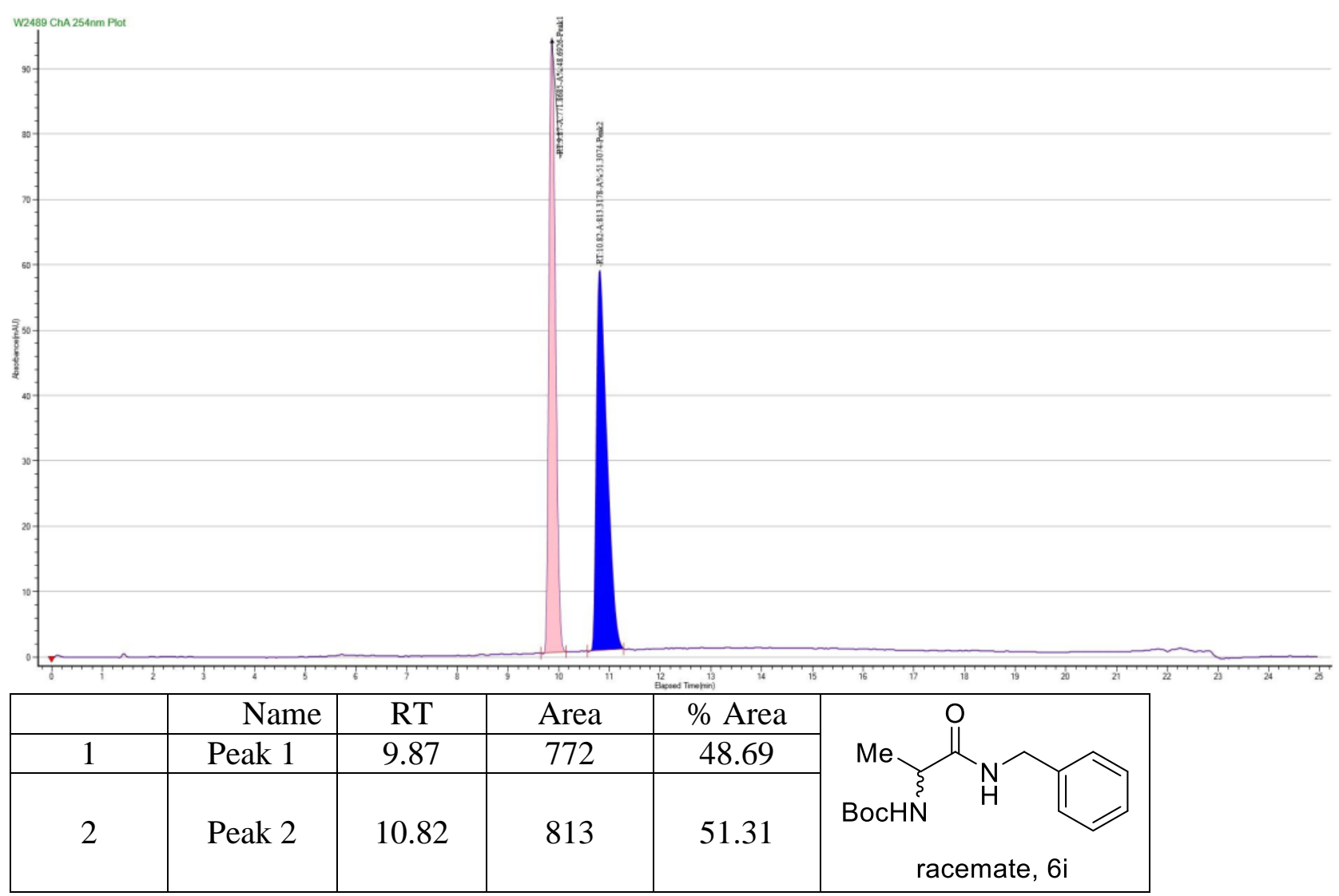

Figure S5. Chromatogram of product $6 \mathbf{i}$ (racemate). 


\begin{tabular}{|c|c|c|c|}
\hline Sample Name: & D-1 & Acquired By: & System \\
\hline Sample Type: & Standard & & \\
\hline Vial: & $1: B, 4$ & Acq. Method Set: & $\begin{array}{l}\mathrm{A}: \mathrm{CO}_{2} \mathrm{~B}: \mathrm{MeOH} \text { (adding } 0.2 \% \\
\text { TFA) } \\
0-20 \mathrm{~min}: 28 \%-58 \% \mathrm{MeOH} \text { (adding } \\
0.2 \% \text { TFA); 20.01-25min: } 28 \% \\
\mathrm{MeOH} \text { (adding 0.2\% TFA) } \\
\text { Column: Chiralcel OD-H 4.6*250nm } \\
\text { 5um }\end{array}$ \\
\hline Injection \#: & 1 & & \\
\hline $\begin{array}{l}\text { Injection } \\
\text { Volume }\end{array}$ & $10.0 \mathrm{ul}$ & Channel Name: & $254.0 \mathrm{~nm}$ \\
\hline $\begin{array}{c}\text { Run Time: } \\
\text { Date acquired: }\end{array}$ & $\begin{array}{c}\text { 25.0 Minutes } \\
\text { 2020/6/22 18:01:19 }\end{array}$ & & \\
\hline $\begin{array}{c}\text { Date } \\
\text { Processed: }\end{array}$ & 2020/6/23 09:10:44 & & \\
\hline
\end{tabular}

Auto-Scaled Chromatogram

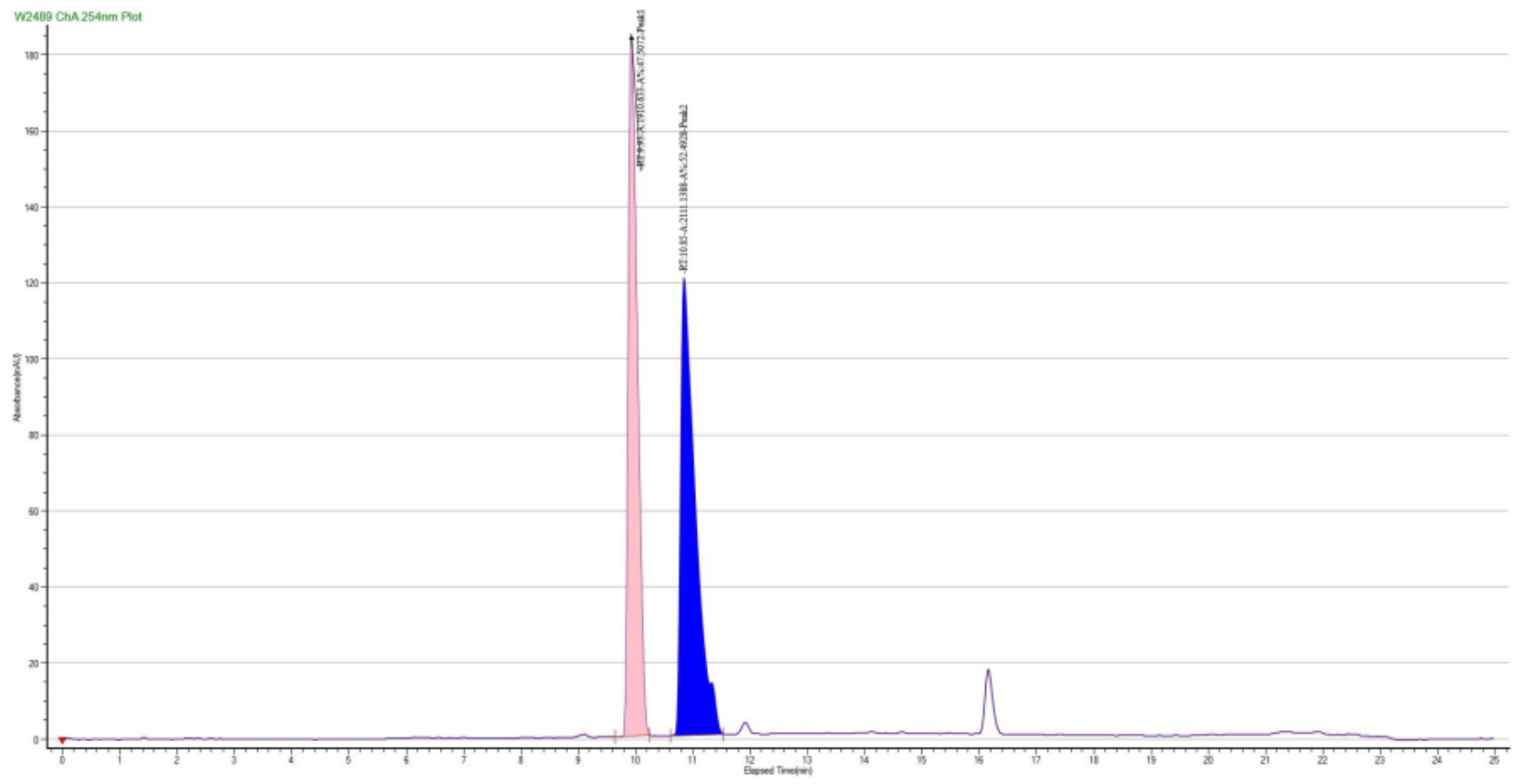

\begin{tabular}{|c|c|c|c|c|c|}
\hline & Name & RT & Area & \% Area & \multirow{2}{*}{ Me } \\
\cline { 1 - 4 } 2 & Peak 1 & 9.93 & 1911 & 47.51 \\
& Peak 2 & 10.85 & 2111 & 52.49 \\
\hline
\end{tabular}

Figure S6 Chromatogram of product $6 \mathbf{i}$. 


\begin{tabular}{|c|c|c|c|}
\hline Sample Name: & DL-2 & Acquired By: & System \\
\hline Sample Type: & Standard & & \\
\hline Vial: & $1: \mathrm{B}, 1$ & Acq. Method Set: & $\begin{array}{l}\mathrm{A}: \mathrm{CO}_{2} \mathrm{~B}: \mathrm{MeOH} \text { (adding } 0.2 \% \\
\text { TFA) } \\
0-20 \mathrm{~min}: 28 \%-58 \% \mathrm{MeOH} \text { (adding } \\
0.2 \% \text { TFA); 20.01-25min: } 28 \% \\
\mathrm{MeOH} \text { (adding 0.2\% TFA) } \\
\text { Column: Chiralcel OD-H 4.6*250nm } \\
\text { 5um }\end{array}$ \\
\hline Injection \#: & 1 & & \\
\hline $\begin{array}{l}\text { Injection } \\
\text { Volume }\end{array}$ & $20.0 \mathrm{ul}$ & Channel Name: & $254.0 \mathrm{~nm}$ \\
\hline $\begin{array}{c}\text { Run Time: } \\
\text { Date acquired: }\end{array}$ & $\begin{array}{c}\text { 25.0 Minutes } \\
\text { 2020/6/22 17:01:12 }\end{array}$ & & \\
\hline $\begin{array}{c}\text { Date } \\
\text { Processed: }\end{array}$ & 2020/6/23 08:55:22 & & \\
\hline
\end{tabular}

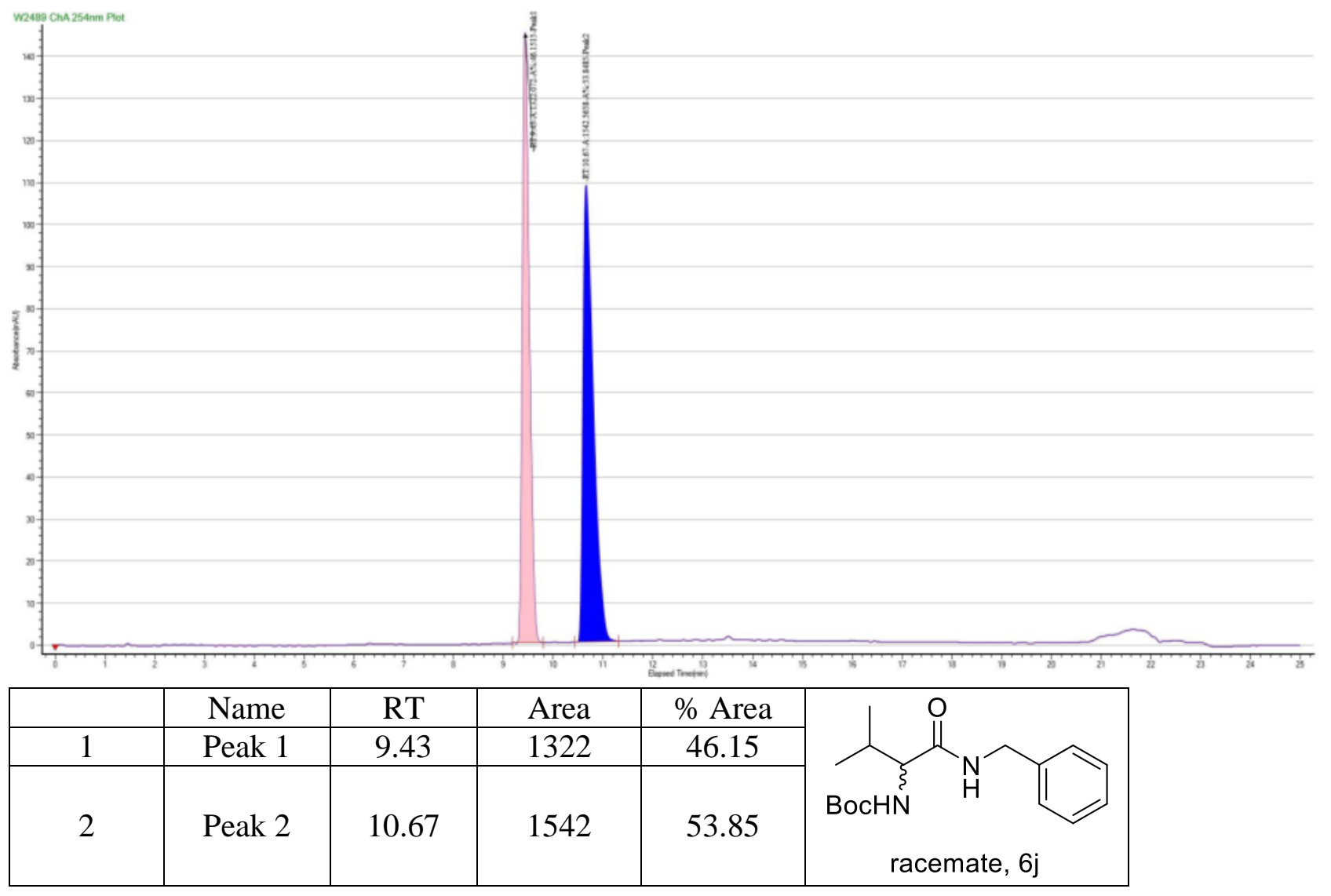

Figure S7 Chromatogram of product $\mathbf{6 j}$ (racemate). 


\begin{tabular}{|c|c|c|c|}
\hline Sample Name: & D-2 & Acquired By: & System \\
\hline Sample Type: & Standard & & \\
\hline Vial: & $1: B, 2$ & Acq. Method Set: & $\begin{array}{l}\mathrm{A}: \mathrm{CO}_{2} \mathrm{~B}: \mathrm{MeOH} \text { (adding } 0.2 \% \\
\text { TFA) } \\
0-20 \mathrm{~min}: 28 \%-58 \% \mathrm{MeOH} \text { (adding } \\
0.2 \% \text { TFA); 20.01-25min: } 28 \% \\
\text { MeOH (adding 0.2\% TFA) } \\
\text { Column: Chiralcel OD-H 4.6*250nm } \\
\text { 5um }\end{array}$ \\
\hline Injection \#: & 1 & & \\
\hline $\begin{array}{l}\text { Injection } \\
\text { Volume }\end{array}$ & $10.0 \mathrm{ul}$ & Channel Name: & $254.0 \mathrm{~nm}$ \\
\hline $\begin{array}{c}\text { Run Time: } \\
\text { Date acquired: }\end{array}$ & $\begin{array}{c}\text { 25.0 Minutes } \\
\text { 2020/6/22 17:31:15 }\end{array}$ & & \\
\hline $\begin{array}{c}\text { Date } \\
\text { Processed: }\end{array}$ & 2020/6/23 08:35:31 & & \\
\hline
\end{tabular}

Auto-Scaled Chromatogram

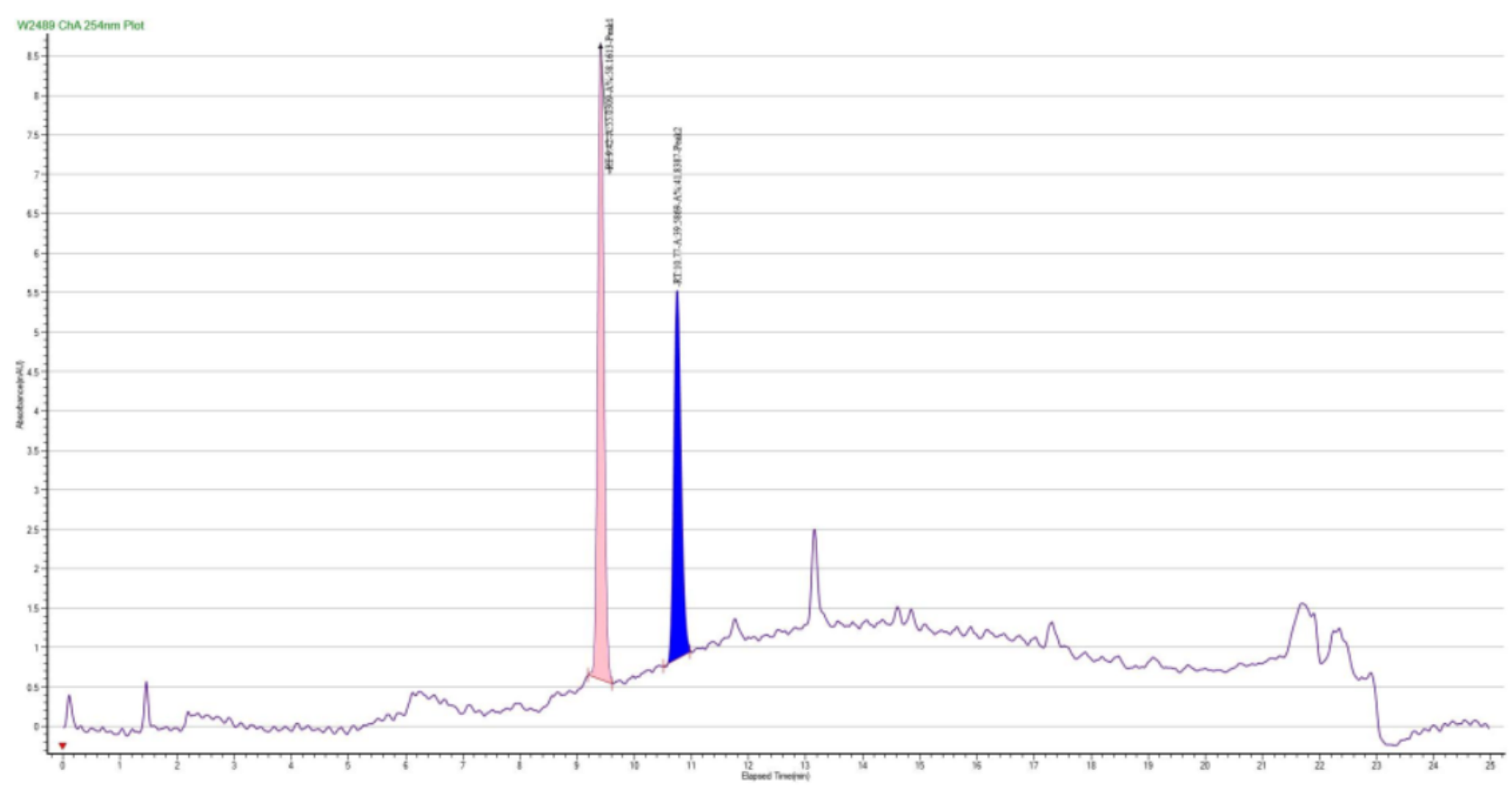

\begin{tabular}{|c|c|c|c|c|c|}
\hline & Name & RT & Area & \% Area & \\
\cline { 1 - 5 } 2 & Peak 1 & 9.42 & 55 & 58.16 \\
\cline { 1 - 2 } & Peak 2 & 10.77 & 40 & 41.84 \\
\hline
\end{tabular}

Figure S8. Chromatogram of product $\mathbf{6 j}$. 


\begin{tabular}{|c|c|c|c|}
\hline Sample Name: & & Acquired By: & System \\
\hline Sample Type: & Standard & $\begin{array}{l}\text { Sample Set } \\
\text { Name }\end{array}$ & DL-3 \\
\hline Vial: & $1: \mathrm{A}, 2$ & Acq. Method Set: & $\begin{array}{l}\mathrm{A}: \mathrm{CO}_{2} \mathrm{~B}: \mathrm{MeOH} \text { (adding } 0.2 \% \\
\text { TFA) } \\
0-20 \mathrm{~min}: 30 \%-60 \% \mathrm{MeOH} \text { (adding } \\
0.2 \% \text { TFA); 20.01-25min: } 30 \% \\
\text { MeOH (adding 0.2\% TFA) } \\
\text { Column: Chiralcel OD-H 4.6*250nm } \\
\text { 5um }\end{array}$ \\
\hline Injection \#: & 1 & & \\
\hline $\begin{array}{l}\text { Injection } \\
\text { Volume }\end{array}$ & $20.0 \mathrm{ul}$ & Channel Name: & $254.0 \mathrm{~nm}$ \\
\hline $\begin{array}{c}\text { Run Time: } \\
\text { Date acquired: }\end{array}$ & $\begin{array}{c}\text { 25.0 Minutes } \\
\text { 2020/6/22 15:11:17 }\end{array}$ & & \\
\hline $\begin{array}{c}\text { Date } \\
\text { Processed: }\end{array}$ & 2020/6/22 15:55:31 & & \\
\hline
\end{tabular}

Auto-Scaled Chromatogram

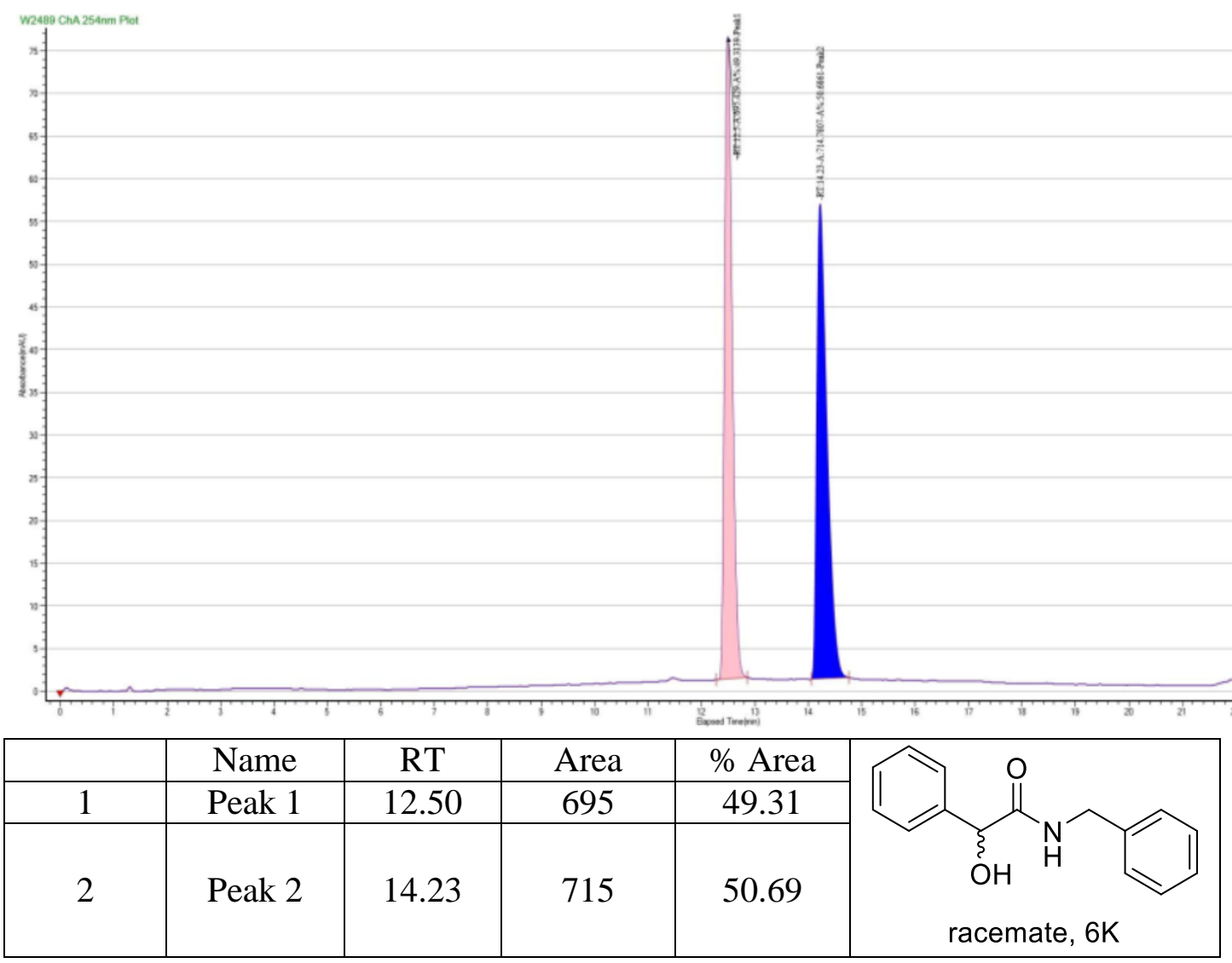

Figure S9. Chromatogram of product $6 \mathbf{k}$ (racemate). 


\begin{tabular}{|c|c|c|c|}
\hline Sample Name: & & Acquired By: & System \\
\hline Sample Type: & Standard & $\begin{array}{l}\text { Sample Set } \\
\text { Name }\end{array}$ & D-3 \\
\hline Vial: & $1: \mathrm{A}, 2$ & Acq. Method Set: & $\begin{array}{l}\mathrm{A}: \mathrm{CO}_{2} \mathrm{~B}: \mathrm{MeOH} \text { (adding } 0.2 \% \\
\text { TFA) } \\
0-20 \mathrm{~min}: 30 \%-60 \% \text { MeOH (adding } \\
0.2 \% \text { TFA); } 20.01-25 \mathrm{~min}: 30 \% \\
\mathrm{MeOH} \text { (adding 0.2\% TFA) } \\
\text { Column: Chiralcel OD-H 4.6*250nm } \\
\text { 5um }\end{array}$ \\
\hline Injection \#: & 1 & & \\
\hline $\begin{array}{l}\text { Injection } \\
\text { Volume }\end{array}$ & $20.0 \mathrm{ul}$ & Channel Name: & $254.0 \mathrm{~nm}$ \\
\hline $\begin{array}{c}\text { Run Time: } \\
\text { Date acquired: }\end{array}$ & $\begin{array}{c}\text { 25.0 Minutes } \\
\text { 2020/6/22 15:45:17 }\end{array}$ & & \\
\hline $\begin{array}{c}\text { Date } \\
\text { Processed: }\end{array}$ & 2020/6/22 16:12:31 & & \\
\hline
\end{tabular}

Auto-Scaled Chromatogram

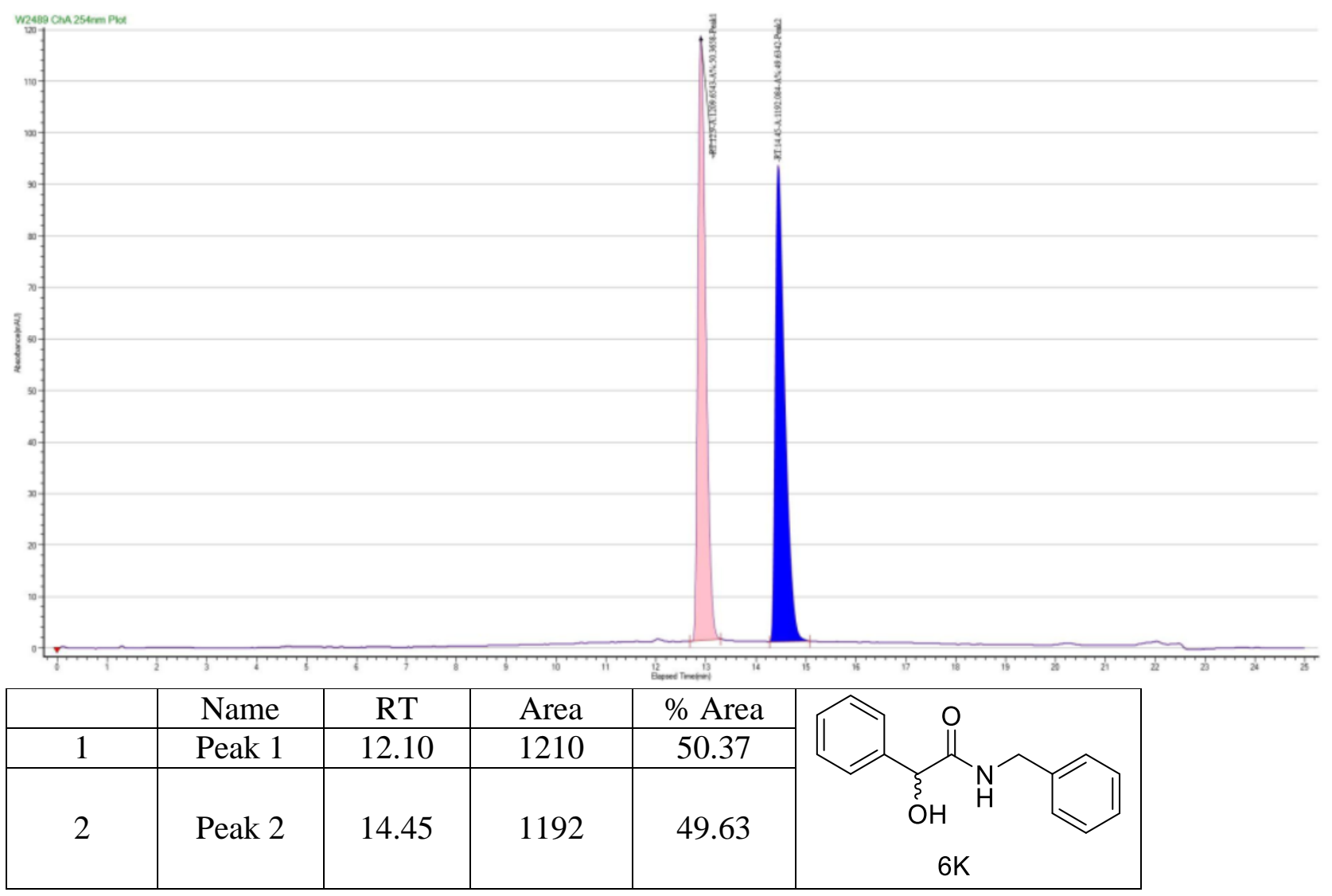

Figure S10. Chromatogram of product $6 \mathbf{k}$. 


\begin{tabular}{|c|c|c|c|}
\hline Sample Name: & DL-3-0702 & Acquired By: & System \\
\hline Sample Type: & Standard & & \\
\hline Vial: & $1: \mathrm{C}, 1$ & Acq. Method Set: & $\begin{array}{l}\mathrm{A}: \mathrm{CO}_{2} \mathrm{~B}: \mathrm{MeOH} \text { (adding } 0.2 \% \\
\text { TFA) } \\
0-20 \mathrm{~min}: 30 \%-60 \% \mathrm{MeOH} \text { (adding } \\
0.2 \% \text { TFA); } 20.01-25 \mathrm{~min}: 30 \% \\
\text { MeOH (adding 0.2\% TFA) } \\
\text { Column: Chiralcel OD-H 4.6*250nm } \\
\text { 5um }\end{array}$ \\
\hline Injection \#: & 1 & & \\
\hline $\begin{array}{l}\text { Injection } \\
\text { Volume }\end{array}$ & $10.0 \mathrm{ul}$ & Channel Name: & $254.0 \mathrm{~nm}$ \\
\hline $\begin{array}{c}\text { Run Time: } \\
\text { Date acquired: }\end{array}$ & $\begin{array}{c}\text { 25.0 Minutes } \\
\text { 2020/7/2 10:13:18 }\end{array}$ & & \\
\hline $\begin{array}{c}\text { Date } \\
\text { Processed: }\end{array}$ & 2020/7/2 10:45:22 & & \\
\hline
\end{tabular}

Auto-Scaled Chromatogram

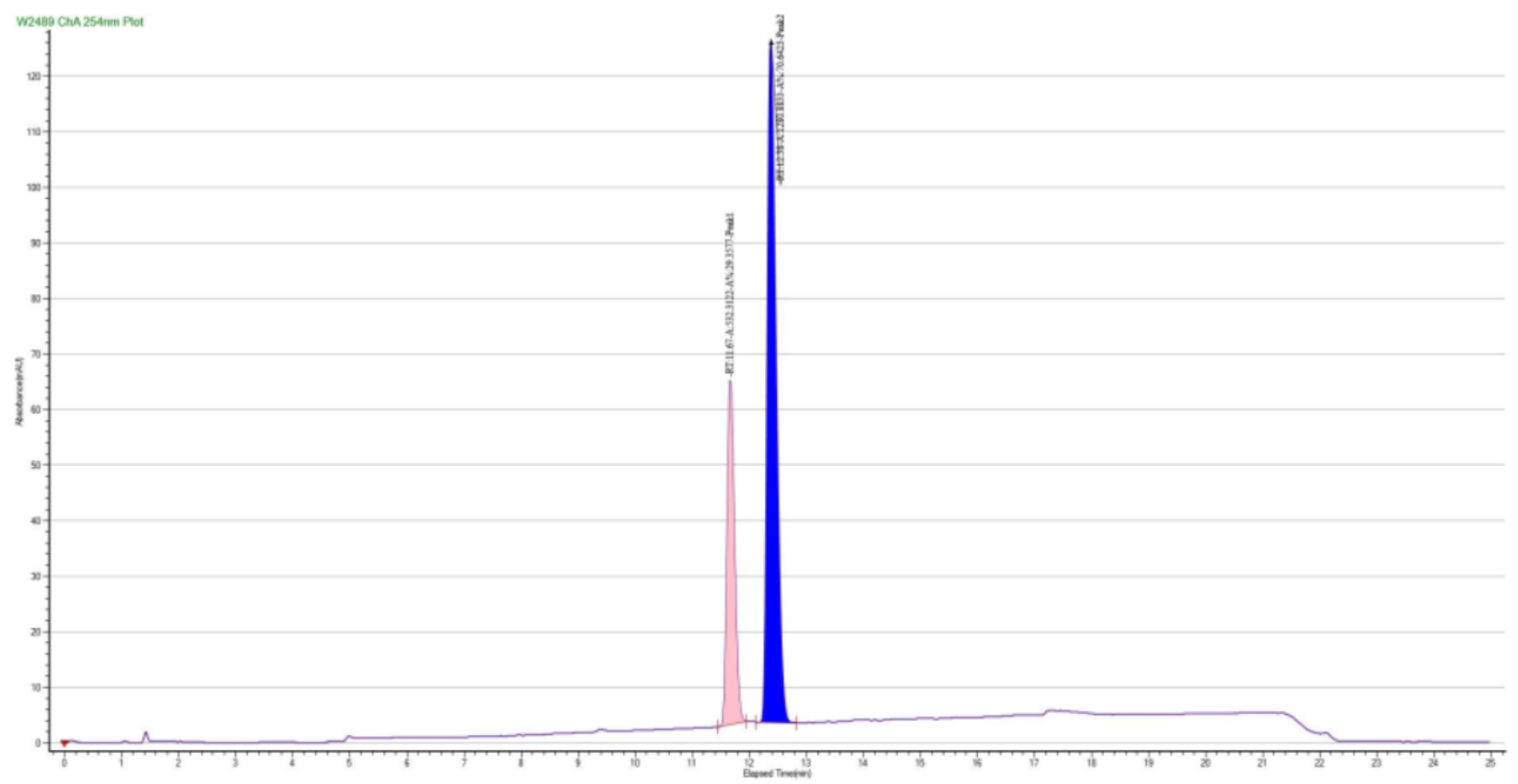

\begin{tabular}{|c|c|c|c|c|c|}
\hline & Name & RT & Area & $\%$ Area \\
\hline 1 & Peak 1 & 11.67 & 532 & 29.36 \\
\hline 2 & Peak 2 & 12.38 & 1281 & 70.64 \\
\hline
\end{tabular}

Figure S11. Chromatogram of product $6 \mathbf{k}$ with $30 \mathrm{~mol} \% 4-\mathrm{CF}_{3}-\mathrm{C}_{6} \mathrm{H}_{4}-\mathrm{OH}$ as additive. 


\section{UV-vis and IR Spectra for Mechanism}

\subsection{UV-vis spectra}

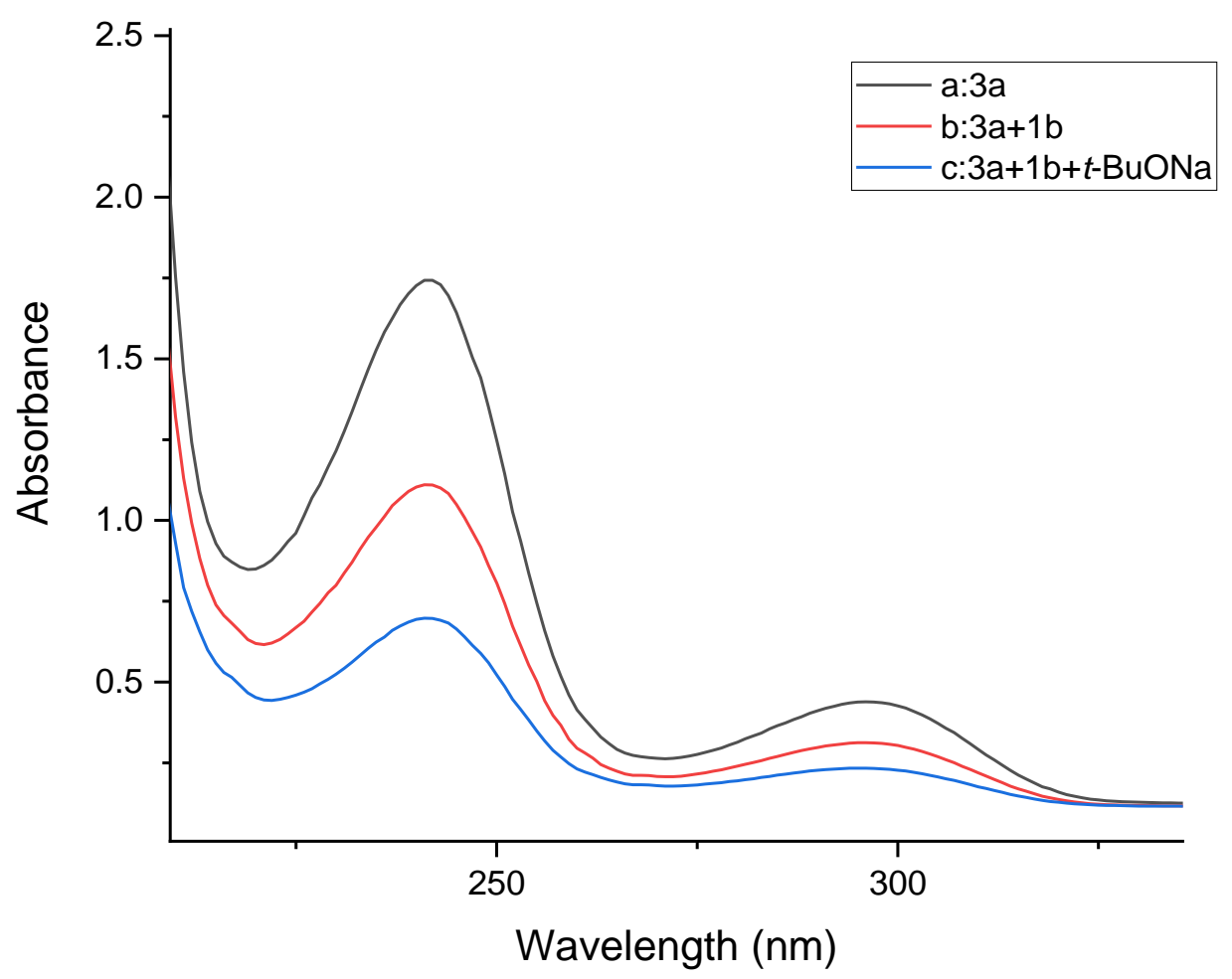

Figure S12. UV-vis spectra of 3a $\left(1.0 \times 10^{-4} \mathrm{~mol} / \mathrm{L}\right)$ in $\mathrm{CH}_{3} \mathrm{CN}$ at $120{ }^{\circ} \mathrm{C}$ for $4 \mathrm{~h}$ under nitrogen atmosphere. (a) $1.2 \mathrm{mmol} \mathrm{3a}$; (b) $1.2 \mathrm{mmol} 3 \mathbf{a}$ and $0.2 \mathrm{mmol} t$-BuONa; (c) $1.2 \mathrm{mmol} \mathrm{3a}, 0.2 \mathrm{mmol} t$-BuONa and $0.01 \mathrm{mmol} \mathbf{1 b}$. 


\subsection{IR-spectrum}
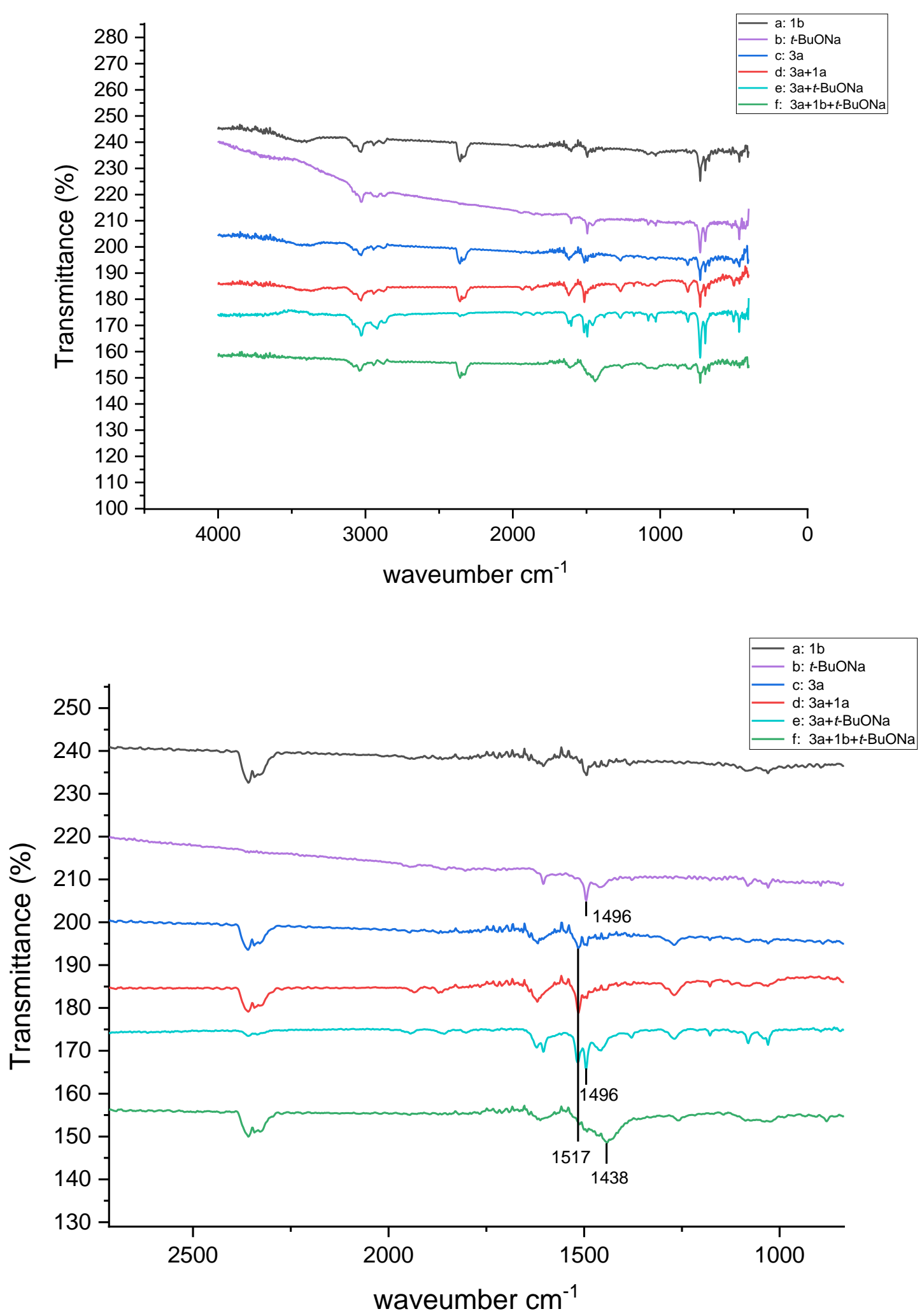

Figure S13. IR-spectrum (KBr) of (a) $0.01 \mathrm{mmol} \mathrm{1b}$; (b) $0.2 \mathrm{mmol} t$-BuONa; (c) $1.2 \mathrm{mmol} \mathrm{3a}$; (d) $1.2 \mathrm{mmol} \mathrm{3a}$ and $0.01 \mathrm{mmol} \mathbf{1 b}$; (e) $1.2 \mathrm{mmol} \mathrm{3a}$ and $0.2 \mathrm{mmol} t$-BuONa; (f) $1.2 \mathrm{mmol} \mathrm{3a}, 0.01 \mathrm{mmol} \mathbf{1 b}$ and $0.2 \mathrm{mmol} t$ $\mathrm{BuONa}$ in toluene at $120^{\circ} \mathrm{C}$ for $4 \mathrm{~h}$ under nitrogen atmosphere. 


\section{Kinetic Studies}

\section{a. General procedure for typical reaction kinetics}

For the reaction of methyl benzoate $(1 \mathrm{mmol})$, amine $(1.2 \mathrm{mmol})$ with $1 \mathrm{~b}(0.01 \mathrm{mmol})$ in $1.5 \mathrm{~mL}$ toluene:

Methyl benzoate $(1 \mathrm{mmol})$, amine $(1.2 \mathrm{mmol}), 1 \mathrm{~b}$ catalyst $(0.01 \mathrm{mmol}), t$-BuONa $(0.2 \mathrm{mmol})$ and were added to a $25 \mathrm{ml}$ of Schlenk tube that was equipped with a magnetic stirrer. The reaction tube was vacuumed and then filled with nitrogen three times. Subsequently, toluene $(1.5 \mathrm{~mL})$ was added to the reaction tube. The reactor heated at $120^{\circ} \mathrm{C}$ taken out at $5,10,15,20,25,30,60,120,240,360$ minutes. Calculate the yield by GC to get the molar concentrations of reactants. A duplicate reaction was also run under otherwise identical conditions and an average value was taken for each time point. The yields in molar concentrations are presented in Table S7. The molar concentrations of the product 4a were plotted against the reaction time to obtain a typical reaction kinetic profile.

Table S7. The molar concentration of product $\mathbf{4 a}$ at different time interval

\begin{tabular}{|c|c|}
\hline Time $(\mathrm{s})$ & Yield of 4a $(\mathrm{M})$ \\
\hline $300 \mathrm{~s}$ & 0.053533 \\
\hline $600 \mathrm{~s}$ & 0.113652 \\
\hline $900 \mathrm{~s}$ & 0.156593 \\
\hline $1200 \mathrm{~s}$ & 0.183463 \\
\hline $1500 \mathrm{~s}$ & 0.244623 \\
\hline $1800 \mathrm{~s}$ & 0.262357 \\
\hline $3600 \mathrm{~s}$ & 0.303652 \\
\hline $7200 \mathrm{~s}$ & 0.326528 \\
\hline $14400 \mathrm{~s}$ & 0.339354 \\
\hline 21600 & 0.351596 \\
\hline
\end{tabular}

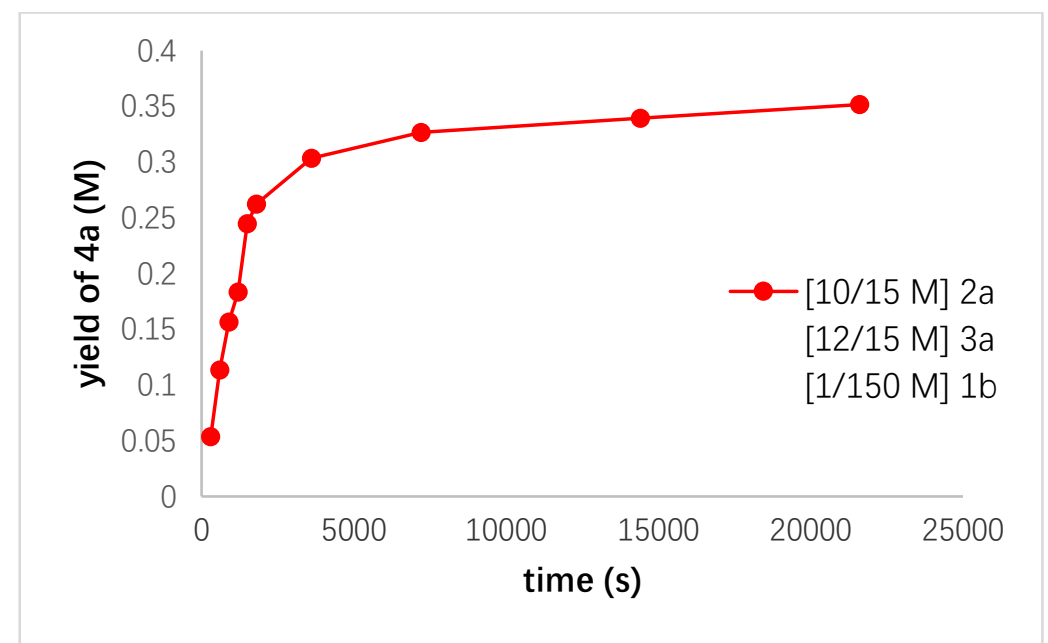

Figure S14. Reaction profile of the amount of amide $4 \mathbf{a}$ formed.

b. General procedure to determine the dependence of reaction rate on the concentration of methyl 


\section{benzoate}

For the reaction of methyl benzoate $(1 \mathrm{mmol})$, amine $(1.2 \mathrm{mmol})$ with $1 \mathrm{~b}(0.01 \mathrm{mmol})$ in $1.5 \mathrm{~mL}$ toluene:

Methyl benzoate $(1 \mathrm{mmol})$, amine $(1.2 \mathrm{mmol}), 1 \mathrm{~b}$ catalyst $(0.01 \mathrm{mmol}), t$ - $\mathrm{BuONa}(0.2 \mathrm{mmol})$ and were added to a $25 \mathrm{ml}$ of Schlenk tube that was equipped with a magnetic stirrer. The reaction tube was vacuumed and then filled with nitrogen three times. Subsequently, toluene $(1.5 \mathrm{~mL})$ was added to the reaction tube. The reactor heated at $120^{\circ} \mathrm{C}$ taken out at $5,10,15,20,25$ minutes. Calculate the yield by GC to get the molar concentrations of reactants. A duplicate reaction was also run under otherwise identical conditions and an average value was taken for each time point. The yields in molar concentrations are presented in Table S8. The molar concentrations of the product $\mathbf{4 a}$ were plotted against the reaction time to obtain a typical reaction kinetic profile.

For the reaction of methyl benzoate $(0.8 \mathrm{mmol})$, amine $(1.2 \mathrm{mmol})$ with $1 \mathrm{~b}(0.01 \mathrm{mmol})$ in $1.5 \mathrm{~mL}$ toluene: The procedure for this reaction was the same as above but instead of methyl benzoate ( $1 \mathrm{mmol})$, methyl benzoate $(0.8 \mathrm{mmol})$ were added in the reaction.

For the reaction of methyl benzoate $(0.6 \mathrm{mmol})$, amine $(1.2 \mathrm{mmol})$ with $1 \mathrm{~b}(0.01 \mathrm{mmol})$ in $1.5 \mathrm{~mL}$ toluene: The procedure for this reaction was the same as above but instead of methyl benzoate ( $1 \mathrm{mmol})$, methyl benzoate $(0.6 \mathrm{mmol})$ were added in the reaction.

For the reaction of methyl benzoate $(0.4 \mathrm{mmol})$, amine $(1.2 \mathrm{mmol})$ with $1 \mathrm{~b}(0.01 \mathrm{mmol})$ in $1.5 \mathrm{~mL}$ toluene: The procedure for this reaction was the same as above but instead of methyl benzoate ( $1 \mathrm{mmol})$, methyl benzoate $(0.4 \mathrm{mmol})$ were added in the reaction.

For the reaction of methyl benzoate $(0.2 \mathrm{mmol})$, amine $(1.2 \mathrm{mmol})$ with $1 \mathrm{~b}(0.01 \mathrm{mmol})$ in $1.5 \mathrm{~mL}$ toluene: The procedure for this reaction was the same as above but instead of methyl benzoate ( $1 \mathrm{mmol})$, methyl benzoate $(0.2 \mathrm{mmol})$ were added in the reaction.

The percentage yields of the product $\mathbf{4 a}$ were calculated by GC to get the molar concentrations of reactants. A duplicate reaction was also run under otherwise identical conditions and an average value was taken for each time point. The molar concentration of product $\mathbf{4 a}$ was plotted against the reaction time and the slope of linear portion of the curve was used to determine the initial rates of the reaction. The table showing molar concentration of product 4a in different concentration of methyl benzoate, graph showing the rate at different concentration of methyl benzoate, table with $k_{i n}$ in value and the graph showing $k_{\text {in }}$ in versus [2a] are shown below.

Table S8. The molar concentration of product 4a in different concentration of methyl benzoate at different time interval

\begin{tabular}{|c|c|c|c|c|c|}
\hline Time $(\mathrm{s})$ & $2 \mathrm{a}$ & $2 \mathrm{a}$ & $2 \mathrm{a}$ & $2 \mathrm{a}$ & $2 \mathrm{a}$ \\
\hline & $10 / 15 \mathrm{M}$ & $8 / 15 \mathrm{M}$ & $6 / 15 \mathrm{M}$ & $4 / 15 \mathrm{M}$ & $2 / 15 \mathrm{M}$ \\
\hline $300 \mathrm{~s}$ & 0.053533 & 0.047212 & 0.032038 & 0.028316 & 0.024633 \\
\hline $600 \mathrm{~s}$ & 0.113652 & 0.077104 & 0.063252 & 0.054327 & 0.040315 \\
\hline $900 \mathrm{~s}$ & 0.156593 & 0.110768 & 0.089234 & 0.075389 & 0.056963 \\
\hline $1200 \mathrm{~s}$ & 0.183463 & 0.152332 & 0.113032 & 0.100592 & 0.066853 \\
\hline $1500 \mathrm{~s}$ & 0.244623 & 0.189456 & 0.152326 & 0.125316 & 0.083423 \\
\hline
\end{tabular}

Table S9. The $k_{\text {in }}$ value of product $4 \mathbf{a}$ in different concentration of methyl benzoate.

\begin{tabular}{|c|c|}
\hline $2 \mathrm{a}(\mathrm{M})$ & $k_{\text {in }} \mathrm{M} \mathrm{s}^{-1}$ \\
\hline $2 / 15$ & $4.8039 \times 10^{-5}$ \\
\hline $4 / 15$ & $8.0088 \times 10^{-5}$ \\
\hline $6 / 15$ & $9.6785 \times 10^{-5}$ \\
\hline $8 / 15$ & $1.1991 \times 10^{-4}$ \\
\hline $10 / 15$ & $1.5066 \times 10^{-4}$ \\
\hline
\end{tabular}




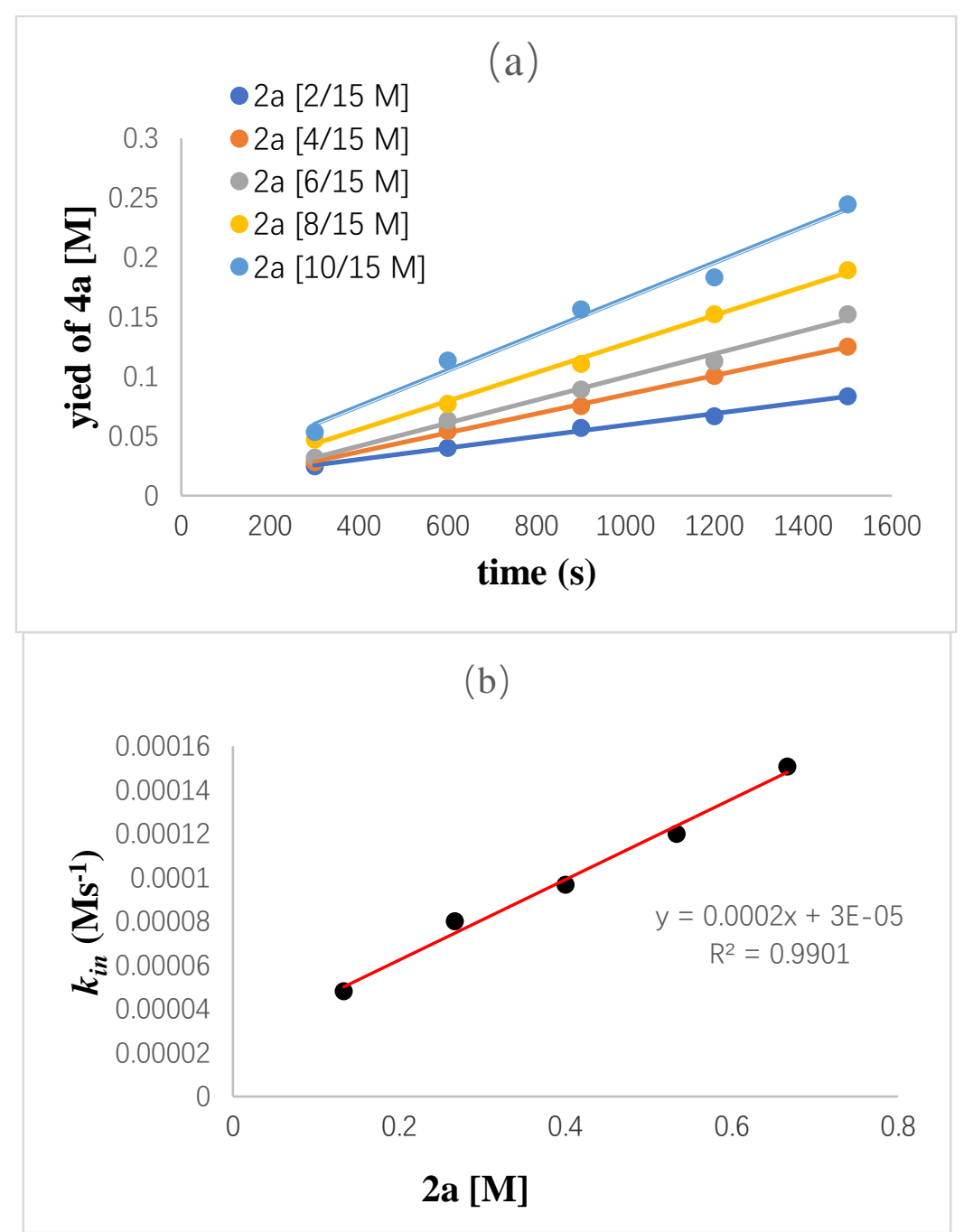

Figure S15. (a) Plot of the rise of product $4 \mathbf{a}$ from the reaction of $\mathbf{3 a}(1.2 \mathrm{mmol}), \mathbf{1 b}(0.01 \mathrm{mmol})$ with $0.20 \mathrm{mmol}$, $0.40 \mathrm{mmol}, 0.60 \mathrm{mmol}, 0.80 \mathrm{mmol}$ and $1.00 \mathrm{mmol}$ of methyl benzoate in $1.5 \mathrm{~mL}$ toluene at different time. (b) Plot of $k_{\text {in }}$ versus [2a] from the reaction of $\mathbf{3 a}(1.2 \mathrm{mmol}), \mathbf{1 b}(0.01 \mathrm{mmol})$ with $0.20 \mathrm{mmol}, 0.40 \mathrm{mmol}, 0.60 \mathrm{mmol}, 0.80$ $\mathrm{mmol}$ and $1.00 \mathrm{mmol}$ of methyl benzoate in $1.5 \mathrm{~mL}$ toluene. 


\section{c. General procedure to determine the dependence of reaction rate on the concentration of amine}

For the reaction of methyl benzoate $(1 \mathrm{mmol})$, amine $(1.2 \mathrm{mmol})$ with $1 \mathrm{~b}(0.01 \mathrm{mmol})$ in $1.5 \mathrm{~mL}$ toluene:

Methyl benzoate $(1 \mathrm{mmol})$, amine $(1.2 \mathrm{mmol}), 1 \mathrm{~b}$ catalyst $(0.01 \mathrm{mmol}), t$-BuONa $(0.2 \mathrm{mmol})$ and were added to a $25 \mathrm{ml}$ of Schlenk tube that was equipped with a magnetic stirrer. The reaction tube was vacuumed and then filled with nitrogen three times. Subsequently, toluene $(1.5 \mathrm{~mL})$ was added to the reaction tube. The reactor heated at $120^{\circ} \mathrm{C}$ taken out at $5,10,15,20,25$ minutes. Calculate the yield by GC to get the molar concentrations of reactants. A duplicate reaction was also run under otherwise identical conditions and an average value was taken for each time point. The yields in molar concentrations are presented in Table S10. The molar concentrations of the product 4a were plotted against the reaction time to obtain a typical reaction kinetic profile.

For the reaction of methyl benzoate $(1 \mathrm{mmol})$, amine $(1.4 \mathrm{mmol})$ with $1 \mathrm{~b}(0.01 \mathrm{mmol})$ in $1.5 \mathrm{~mL}$ toluene: The procedure for this reaction was the same as above but instead of amine $(1.2 \mathrm{mmol})$, amine $(1.4 \mathrm{mmol})$ were added in the reaction.

For the reaction of methyl benzoate $(1 \mathrm{mmol})$, amine $(1.0 \mathrm{mmol})$ with $1 \mathrm{~b}(0.01 \mathrm{mmol})$ in $1.5 \mathrm{~mL}$ toluene: The procedure for this reaction was the same as above but instead of amine (1.2 mmol), amine $(1.0 \mathrm{mmol})$ were added in the reaction.

For the reaction of methyl benzoate $(1 \mathrm{mmol})$, amine $(0.8 \mathrm{mmol})$ with $1 \mathrm{~b}(0.01 \mathrm{mmol})$ in $1.5 \mathrm{~mL}$ toluene: The procedure for this reaction was the same as above but instead of amine (1.2 mmol), amine $(0.8 \mathrm{mmol})$ were added in the reaction.

The percentage yields of the product $\mathbf{4 a}$ were calculated by GC to get the molar concentrations of reactants. A duplicate reaction was also run under otherwise identical conditions and an average value was taken for each time point. The molar concentration of product $\mathbf{4 a}$ was plotted against the reaction time and the slope of linear portion of the curve was used to determine the initial rates of the reaction. The table showing molar concentration of product 4a in different concentration of amine, graph showing the rate at different concentration of amine, table with $k_{\text {in }}$ in value and the graph showing $k_{\text {in }}$ in versus [3a] are shown below.

Table S10. The molar concentration of product $4 \mathbf{a}$ in different concentration of amine at different time interval

\begin{tabular}{|c|c|c|c|c|}
\hline Time $(\mathrm{s})$ & $3 \mathrm{a}$ & $3 \mathrm{a}$ & $3 \mathrm{a}$ & $3 \mathrm{a}$ \\
\hline & $14 / 15 \mathrm{M}$ & $12 / 15 \mathrm{M}$ & $10 / 15 \mathrm{M}$ & $8 / 15 \mathrm{M}$ \\
\hline $300 \mathrm{~s}$ & 0.054628 & 0.053533 & 0.048268 & 0.054635 \\
\hline $600 \mathrm{~s}$ & 0.116842 & 0.113652 & 0.094586 & 0.121533 \\
\hline $900 \mathrm{~s}$ & 0.163254 & 0.156593 & 0.136479 & 0.157684 \\
\hline $1200 \mathrm{~s}$ & 0.188362 & 0.183463 & 0.168645 & 0.181566 \\
\hline $1500 \mathrm{~s}$ & 0.23856 & 0.244623 & 0.236421 & 0.245865 \\
\hline
\end{tabular}

Table S11. The $k_{\text {in }}$ value of product $\mathbf{4 a}$ in different concentration of amine.

\begin{tabular}{|c|c|}
\hline $3 \mathrm{a}(\mathrm{M})$ & $k_{\text {in }} \mathrm{M} \mathrm{s}^{-1}$ \\
\hline $8 / 15$ & $1.475 \times 10^{-4}$ \\
\hline $10 / 15$ & $1.5012 \times 10^{-4}$ \\
\hline $12 / 15$ & $1.5066 \times 10^{-4}$ \\
\hline $14 / 15$ & $1.4646 \times 10^{-4}$ \\
\hline
\end{tabular}




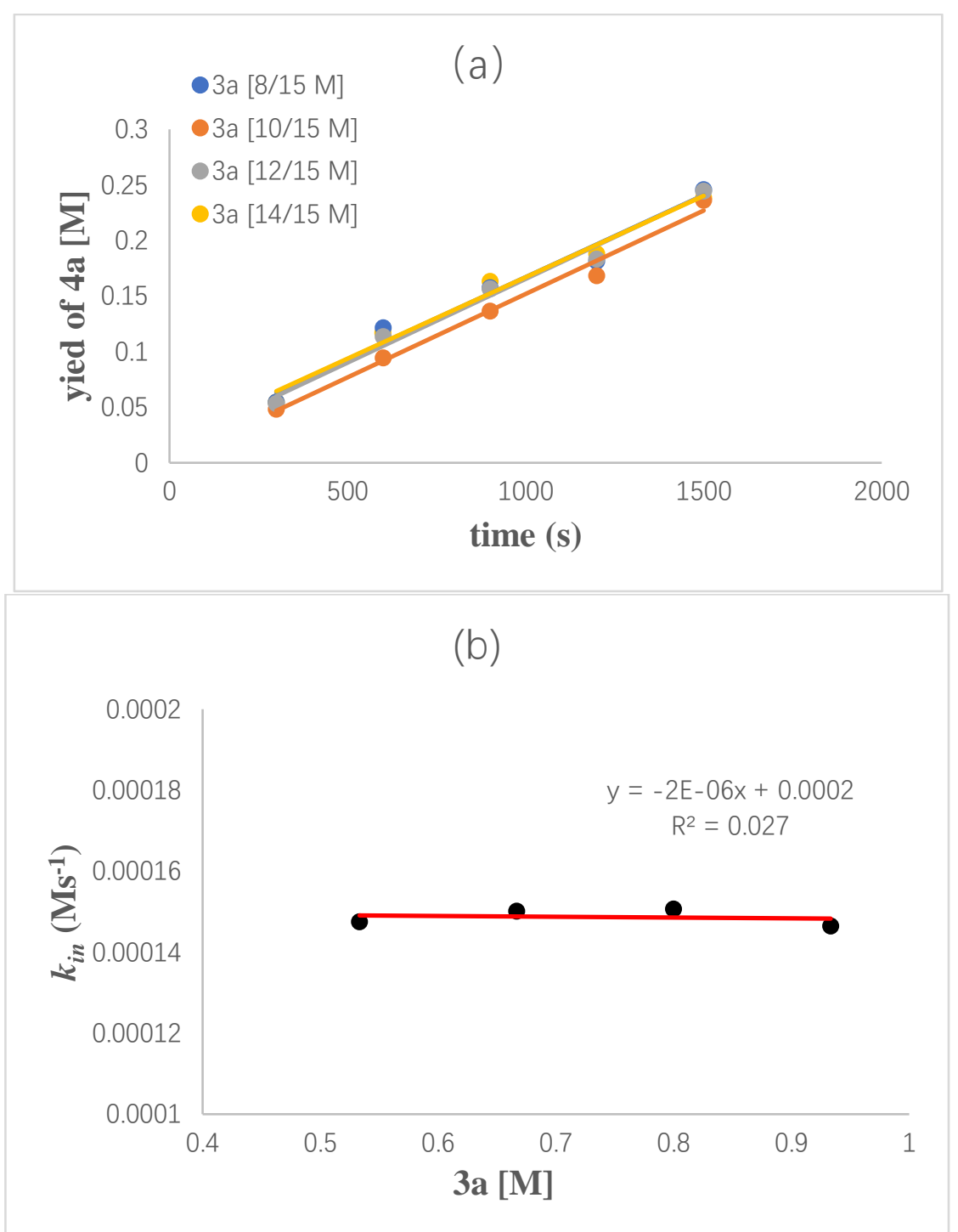

Figure S16. (a) Plot of the rise of product $4 \mathbf{a}$ from the reaction of $\mathbf{2 a}(1.0 \mathrm{mmol}), \mathbf{1 b}(0.01 \mathrm{mmol})$ with $0.80 \mathrm{mmol}$, $1.00 \mathrm{mmol}, 1.20 \mathrm{mmol}$ and $1.40 \mathrm{mmol}$ of amine in $1.5 \mathrm{~mL}$ toluene at different time. (b) Plot of $k_{\text {in }}$ versus [3a] from the reaction of $2 \mathbf{a}(1.0 \mathrm{mmol}), \mathbf{1 b}(0.01 \mathrm{mmol})$ with $0.80 \mathrm{mmol}, 1.00 \mathrm{mmol}, 1.20 \mathrm{mmol}$ and $1.40 \mathrm{mmol}$ of amine in $1.5 \mathrm{~mL}$ toluene. 


\section{d. General procedure to determine the dependence of reaction rate on the concentration of catalyst}

For the reaction of methyl benzoate $(1 \mathrm{mmol})$, amine $(1.2 \mathrm{mmol})$ with $1 \mathrm{~b}(0.01 \mathrm{mmol})$ in $1.5 \mathrm{~mL}$ toluene:

Methyl benzoate $(1 \mathrm{mmol})$, amine $(1.2 \mathrm{mmol}), 1 \mathrm{~b}$ catalyst $(0.01 \mathrm{mmol}), t$ - $\mathrm{BuONa}(0.2 \mathrm{mmol})$ and were added to a $25 \mathrm{ml}$ of Schlenk tube that was equipped with a magnetic stirrer. The reaction tube was vacuumed and then filled with nitrogen three times. Subsequently, toluene $(1.5 \mathrm{~mL})$ was added to the reaction tube. The reactor heated at $120^{\circ} \mathrm{C}$ taken out at $5,10,15,20,25$ minutes. Calculate the yield by GC to get the molar concentrations of reactants. A duplicate reaction was also run under otherwise identical conditions and an average value was taken for each time point. The yields in molar concentrations are presented in Table S12. The molar concentrations of the product 4a were plotted against the reaction time to obtain a typical reaction kinetic profile.

For the reaction of methyl benzoate $(1 \mathrm{mmol})$, amine $(1.4 \mathrm{mmol})$ with $1 \mathrm{~b}(0.008 \mathrm{mmol})$ in $1.5 \mathrm{~mL}$ toluene: The procedure for this reaction was the same as above but instead of $1 \mathrm{~b}(0.01 \mathrm{mmol}), 1 \mathrm{~b}(0.008$ mmol) were added in the reaction.

For the reaction of methyl benzoate $(1 \mathrm{mmol})$, amine $(1.0 \mathrm{mmol})$ with $1 \mathrm{~b}(0.006 \mathrm{mmol})$ in $1.5 \mathrm{~mL}$ toluene: The procedure for this reaction was the same as above but instead of $1 \mathrm{~b}(0.01 \mathrm{mmol}), 1 \mathrm{~b}(0.006$ mmol) were added in the reaction.

For the reaction of methyl benzoate $(1 \mathrm{mmol})$, amine $(0.8 \mathrm{mmol})$ with $1 \mathrm{~b}(0.004 \mathrm{mmol})$ in $1.5 \mathrm{~mL}$ toluene: The procedure for this reaction was the same as above but instead of $1 \mathrm{~b}(0.01 \mathrm{mmol}), 1 \mathrm{~b}(0.004$ mmol) were added in the reaction.

The percentage yields of the product $\mathbf{4 a}$ were calculated by GC to get the molar concentrations of reactants. A duplicate reaction was also run under otherwise identical conditions and an average value was taken for each time point. The molar concentration of product $4 \mathbf{a}$ was plotted against the reaction time and the slope of linear portion of the curve was used to determine the initial rates of the reaction. The table showing molar concentration of product 4a in different concentration of catalyst, graph showing the rate at different concentration of catalyst, table with $k_{\text {in }}$ in value and the graph showing $k_{i n}$ in versus [1b] are shown below.

Table S12. The molar concentration of product $4 \mathbf{a}$ in different concentration of catalyst at different time interval

\begin{tabular}{|c|c|c|c|c|}
\hline Time $(\mathrm{s})$ & $1 \mathrm{~b}$ & $1 \mathrm{~b}$ & $1 \mathrm{~b}$ & $1 \mathrm{~b}$ \\
\hline & $1 / 150 \mathrm{M}$ & $8 / 1500 \mathrm{M}$ & $6 / 1500 \mathrm{M}$ & $4 / 1500 \mathrm{M}$ \\
\hline $300 \mathrm{~s}$ & 0.053533 & 0.045263 & 0.028425 & 0.013863 \\
\hline $600 \mathrm{~s}$ & 0.113652 & 0.068064 & 0.041652 & 0.029215 \\
\hline $900 \mathrm{~s}$ & 0.156593 & 0.099259 & 0.061521 & 0.051691 \\
\hline $1200 \mathrm{~s}$ & 0.183463 & 0.144863 & 0.08725 & 0.069631 \\
\hline $1500 \mathrm{~s}$ & 0.244623 & 0.189966 & 0.115684 & 0.076452 \\
\hline
\end{tabular}

Table S13. The $k_{\text {in }}$ value of product $\mathbf{4 a}$ in different concentration of catalyst.

\begin{tabular}{|c|c|}
\hline $1 \mathrm{~b}(\mathrm{M})$ & $k_{\text {in }} \mathrm{M} \mathrm{s}^{-1}$ \\
\hline $4 / 1500$ & $5.52 \times 10^{-5}$ \\
\hline $6 / 1500$ & $7.337 \times 10^{-5}$ \\
\hline $8 / 1500$ & $1.2207 \times 10^{-4}$ \\
\hline $1 / 150$ & $1.5066 \times 10^{-4}$ \\
\hline
\end{tabular}




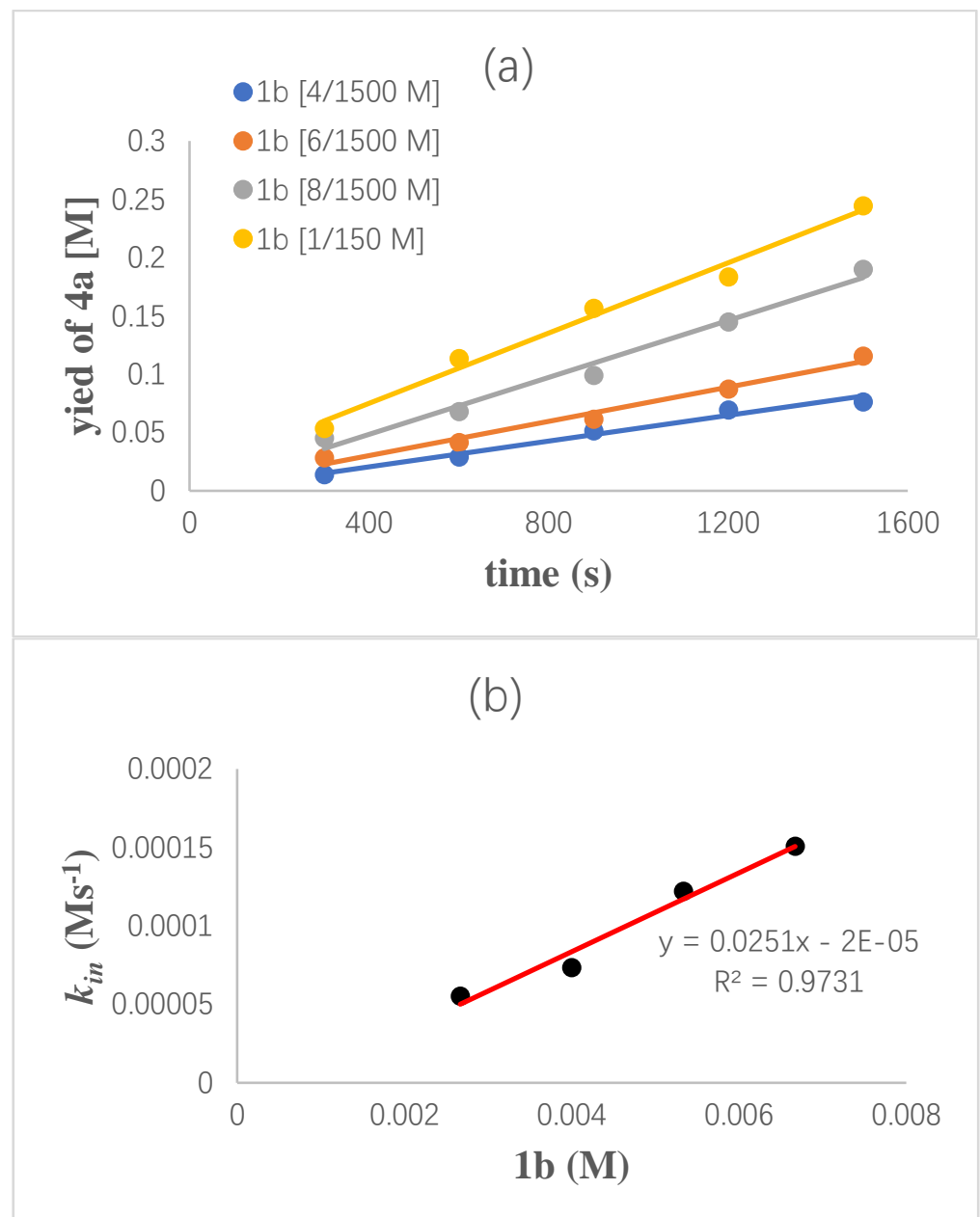

Figure S17. (a) Plot of the rise of product $4 \mathbf{a}$ from the reaction of $\mathbf{2 a}(1.0 \mathrm{mmol}), \mathbf{3 a}(1.2 \mathrm{mmol})$ with $0.004 \mathrm{mmol}$, $0.006 \mathrm{mmol}, 0.008 \mathrm{mmol}$ and $0.010 \mathrm{mmol}$ of catalyst in $1.5 \mathrm{~mL}$ toluene at different time. (b) Plot of $k_{\text {in }}$ versus [1 $\mathbf{1 b}$ ] from the reaction of $\mathbf{2 a}(1.0 \mathrm{mmol}), \mathbf{3 a}(1.2 \mathrm{mmol})$ with $0.004 \mathrm{mmol}, 0.006 \mathrm{mmol}, 0.008 \mathrm{mmol}$ and $0.010 \mathrm{mmol}$ of catalyst in $1.5 \mathrm{~mL}$ toluene. 
10. NMR Spectra of Manganese Complexes
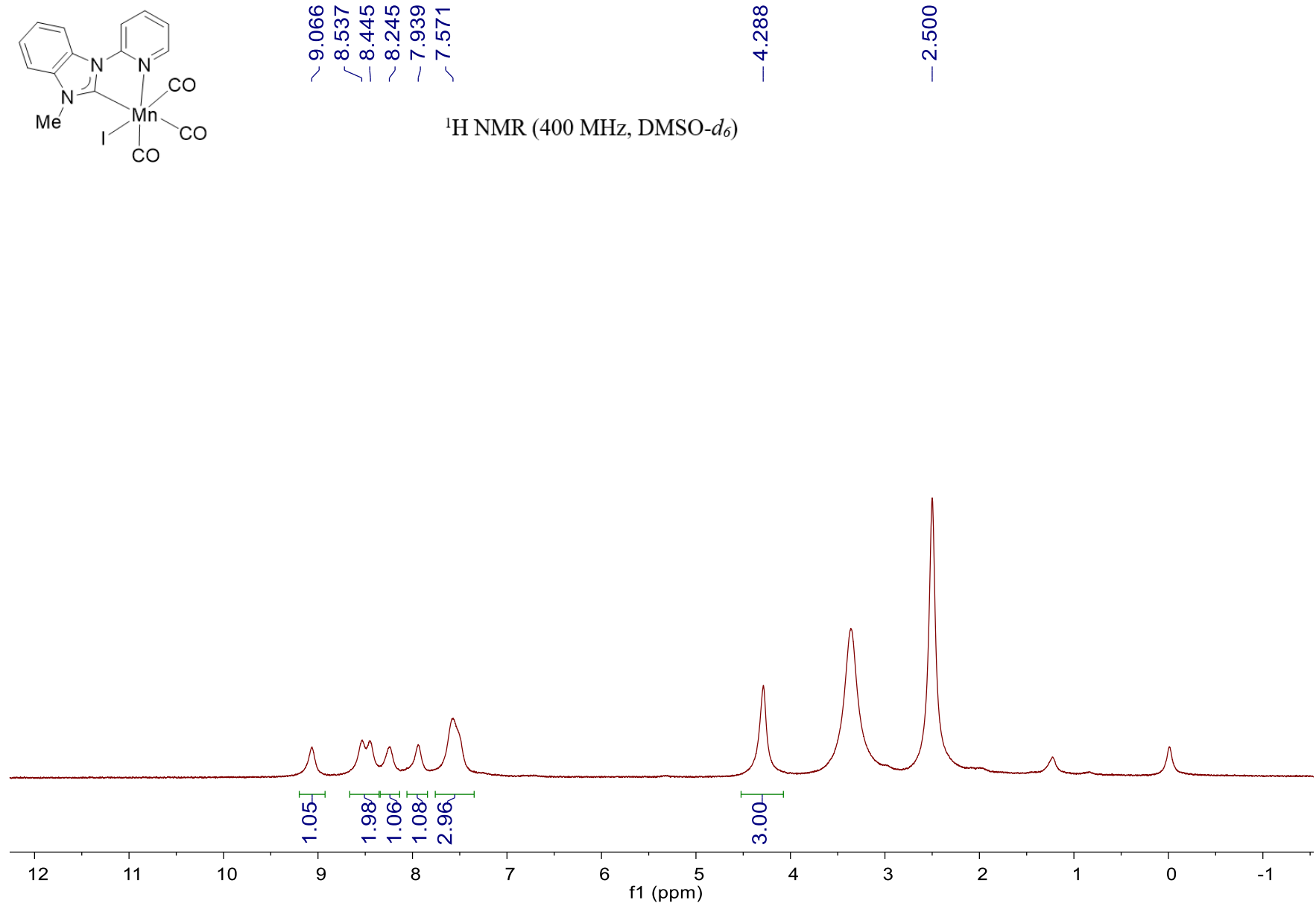

Figure S18. ${ }^{1} \mathrm{H}$ NMR Spectra of Manganese Complex 1a 


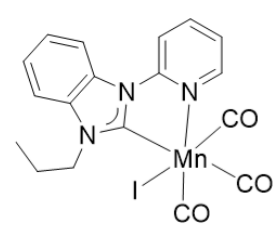

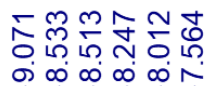

용

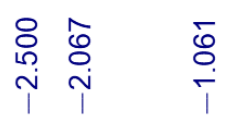

${ }^{1} \mathrm{H}$ NMR (400 MHz, DMSO- $d_{6}$ )

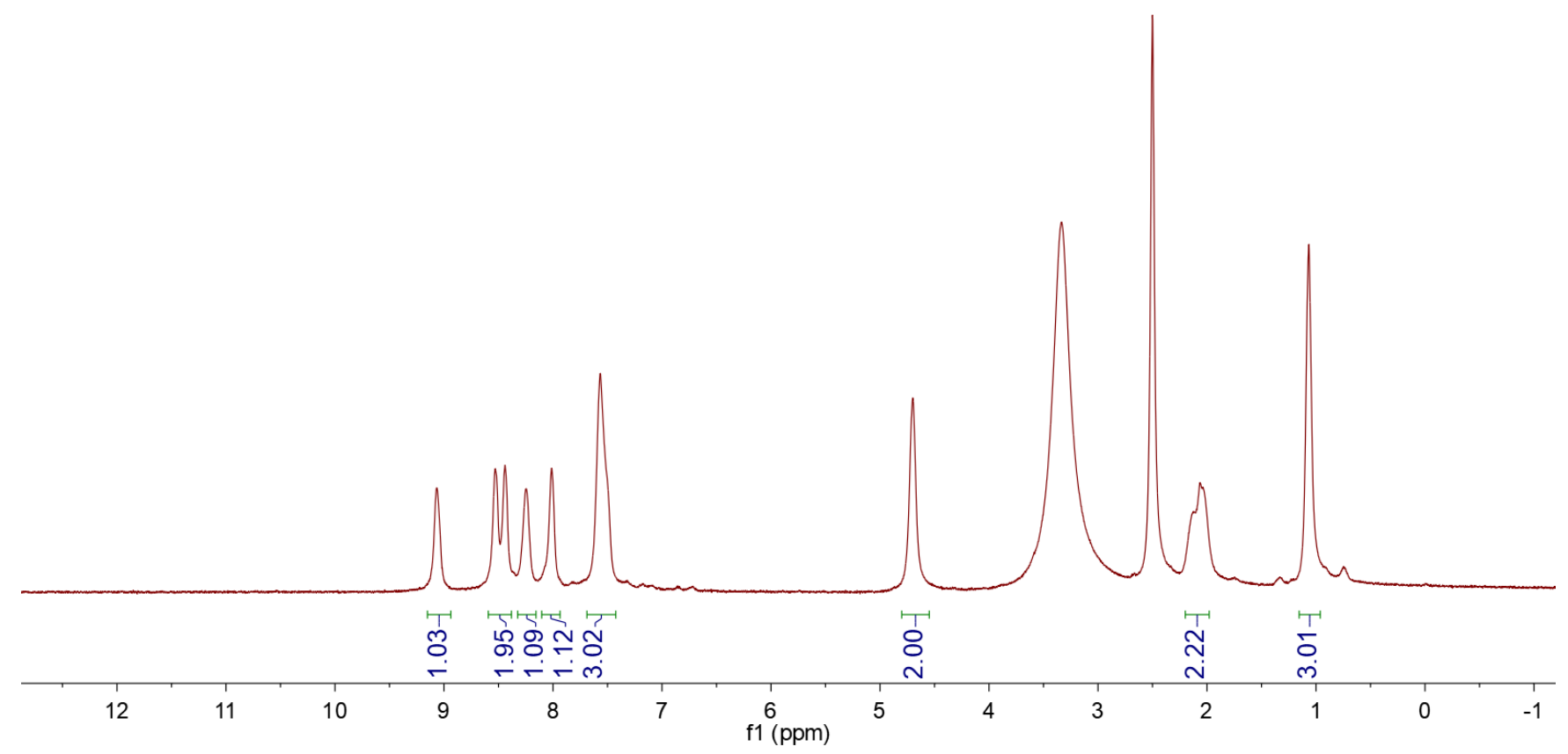

Figure S19. ${ }^{1} \mathrm{H}$ NMR Spectra of Manganese Complex $\mathbf{1 b}$. 

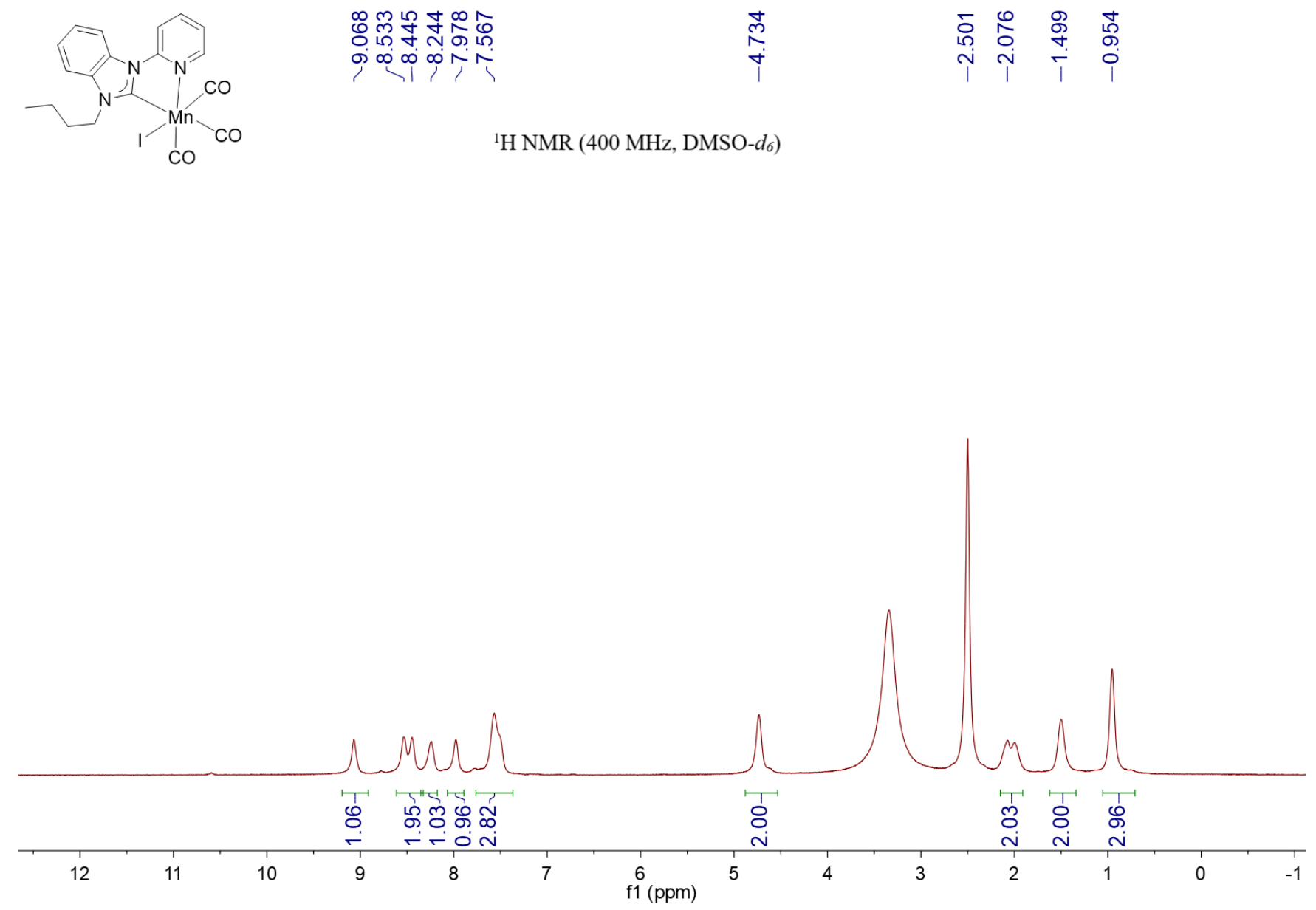

Figure S20. ${ }^{1} \mathrm{H}$ NMR Spectra of Manganese Complex 1c. 

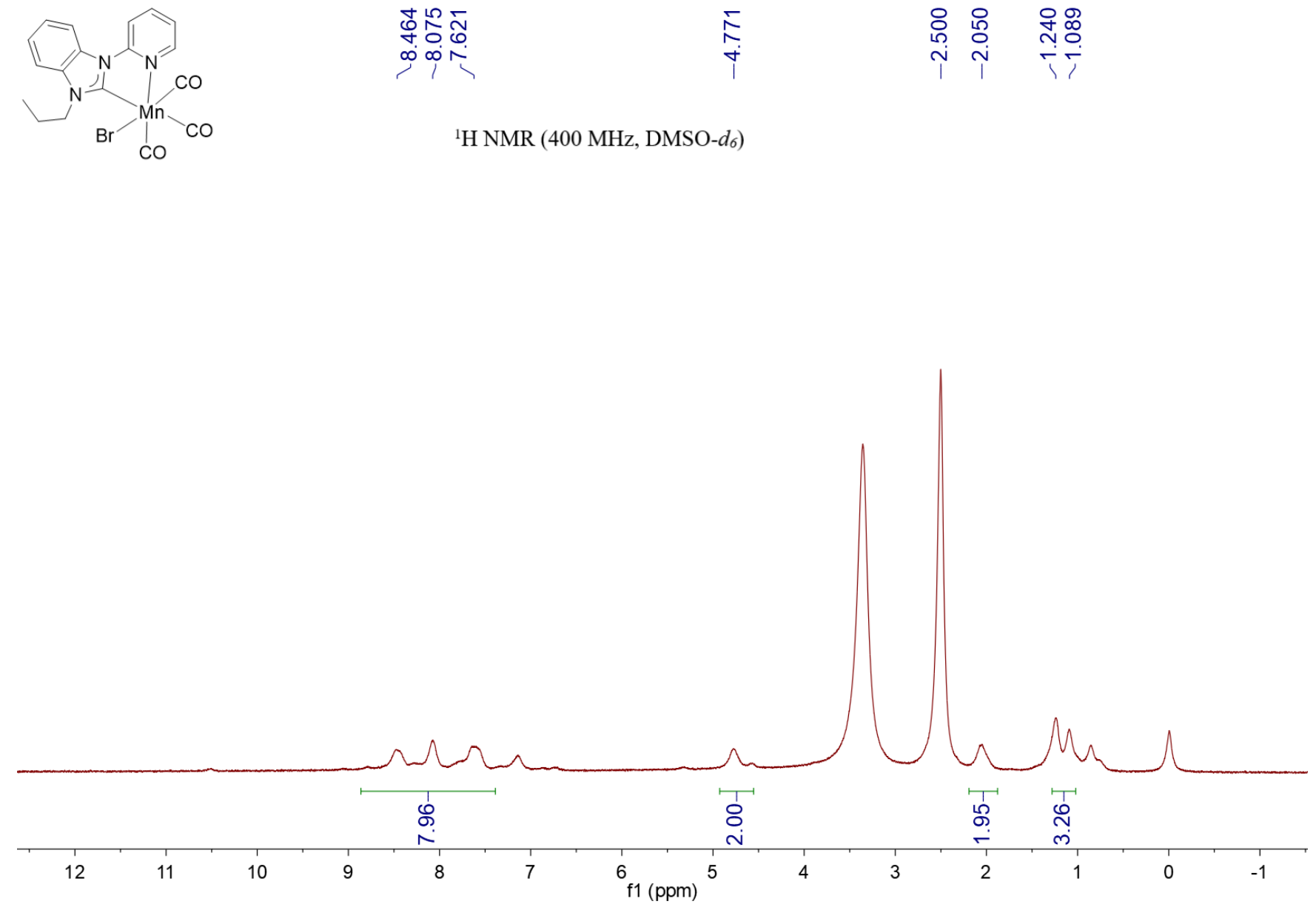

Figure S21. ${ }^{1} \mathrm{H}$ NMR Spectra of Manganese Complex 1d. 

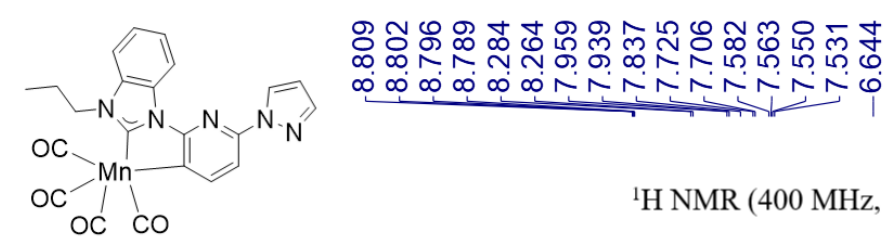

${ }^{1} \mathrm{H}$ NMR (400 MHz, DMSO- $d_{6}$ )

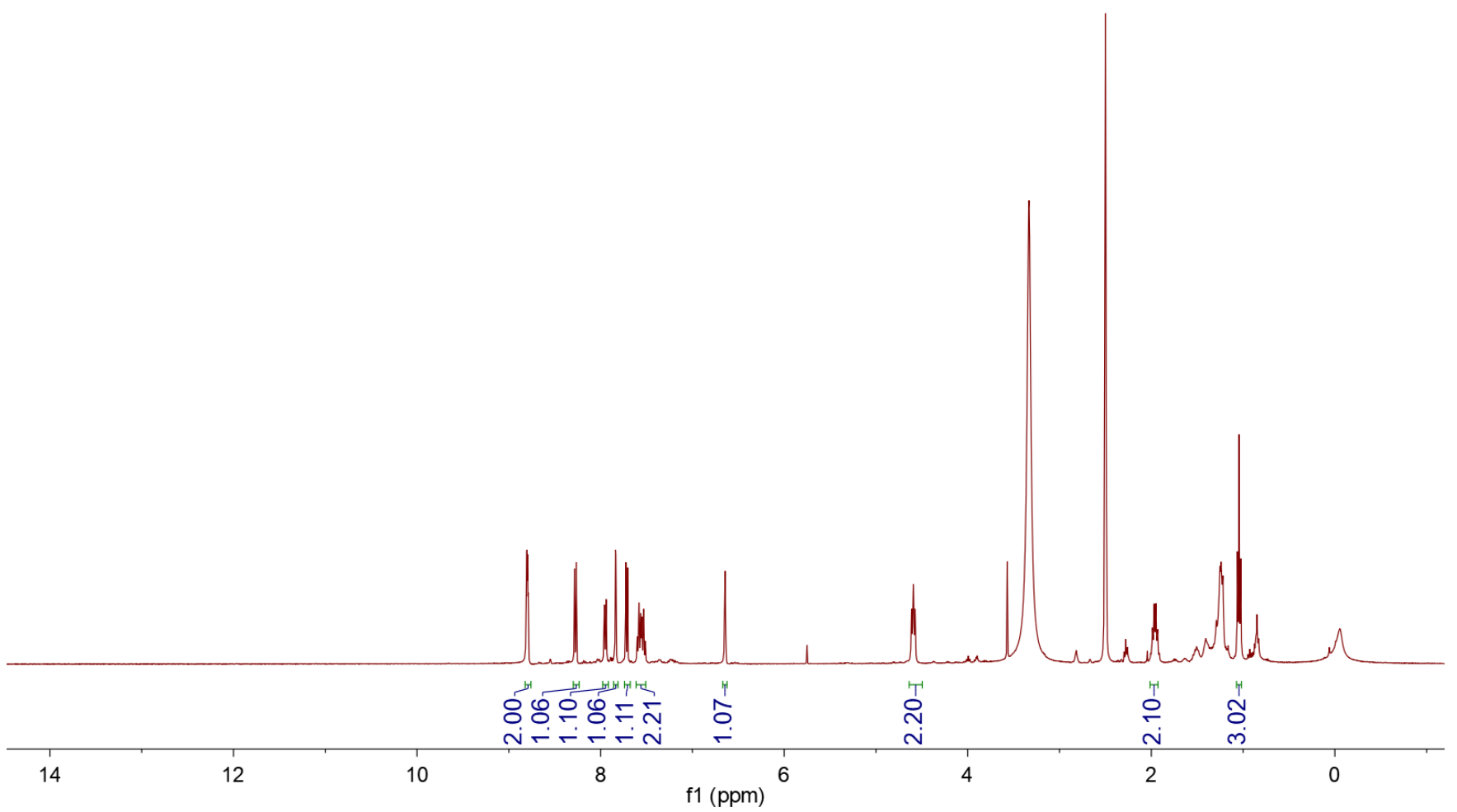

Figure S22. ${ }^{1} \mathrm{H}$ NMR Spectra of Manganese Complex 1 e. 
11. NMR Spectra of Amindes

${ }^{2} \overbrace{n}^{h}$

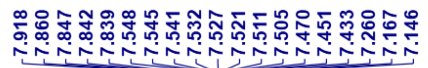

${ }^{1} \mathrm{H} \mathrm{NMR}\left(400 \mathrm{MHz}, \mathrm{CDCl}_{3}\right)$

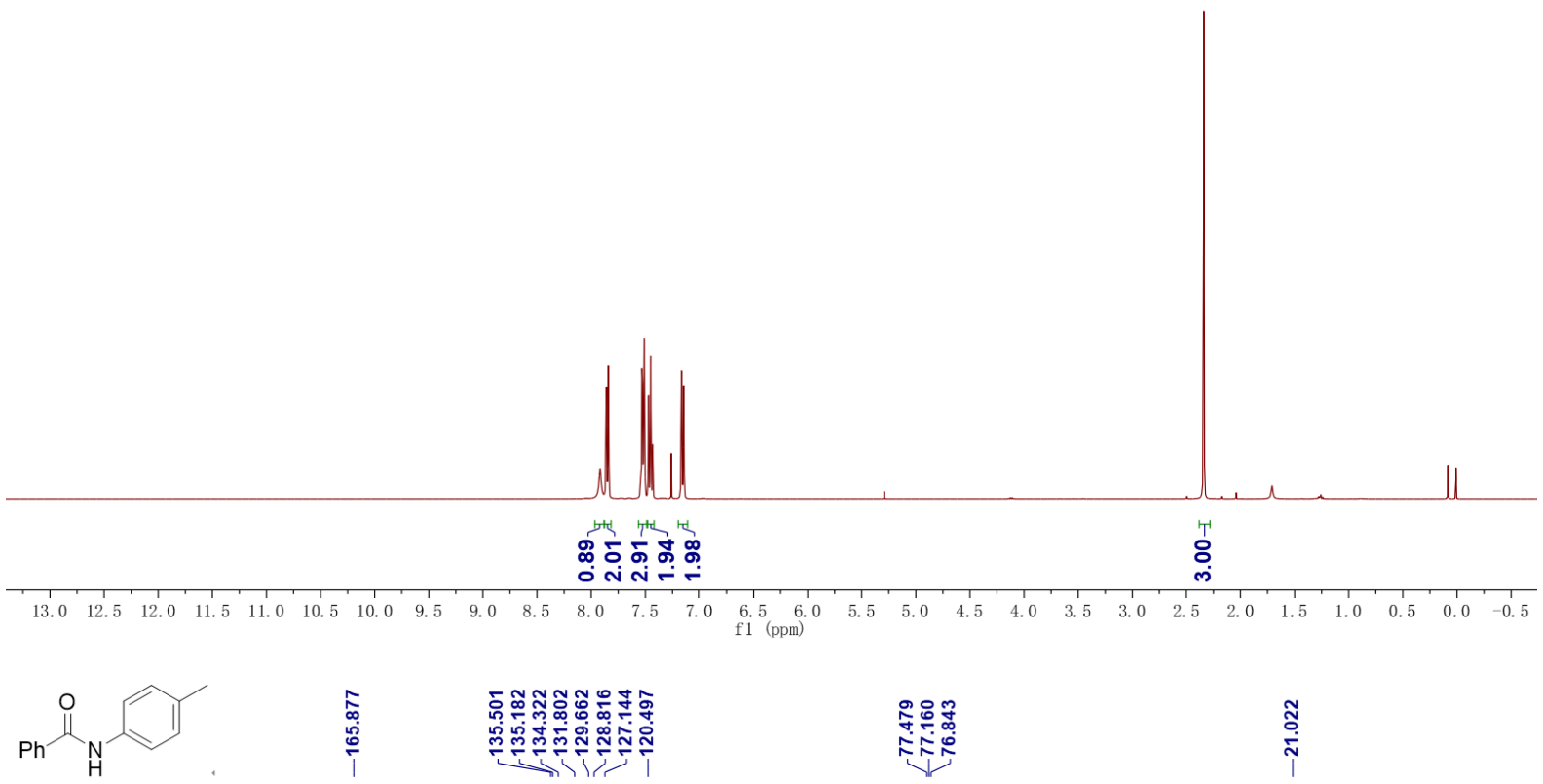

${ }^{13} \mathrm{C}$ NMR $\left(100 \mathrm{MHz}, \mathrm{CDCl}_{3}\right)$

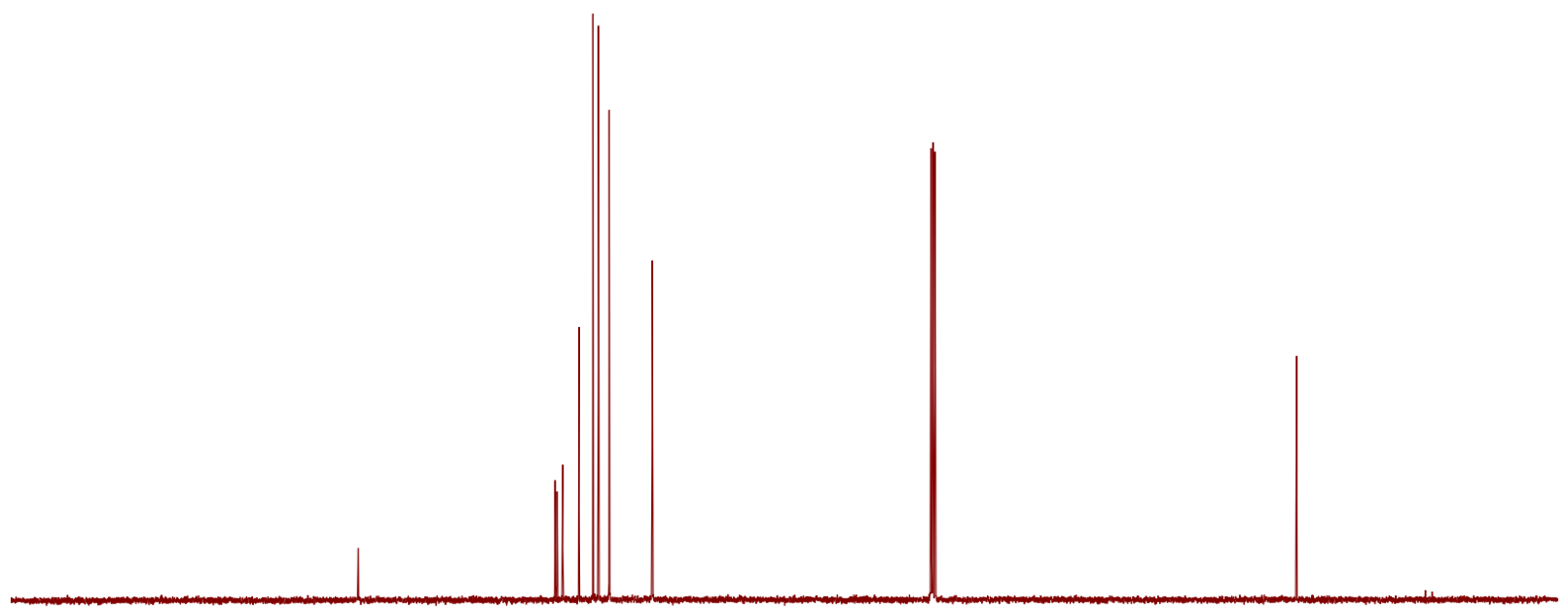

$\begin{array}{lllllllllllll}210 & 200 & 190 & 180 & 170 & 160 & 150 & 140 & 130 & 120 & 110 & 100 & 90\end{array}$

Figure S23. ${ }^{1} \mathrm{H}$ NMR and ${ }^{13} \mathrm{C}\left\{{ }^{1} \mathrm{H}\right\}$ NMR Spectra of $N$-(p-tolyl)benzamide (4a). 
舟

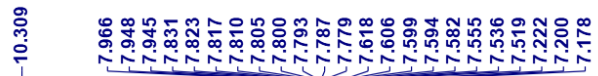

${ }^{1} \mathrm{H}$ NMR (400 MHz, DMSO- $\left.d_{6}\right)$

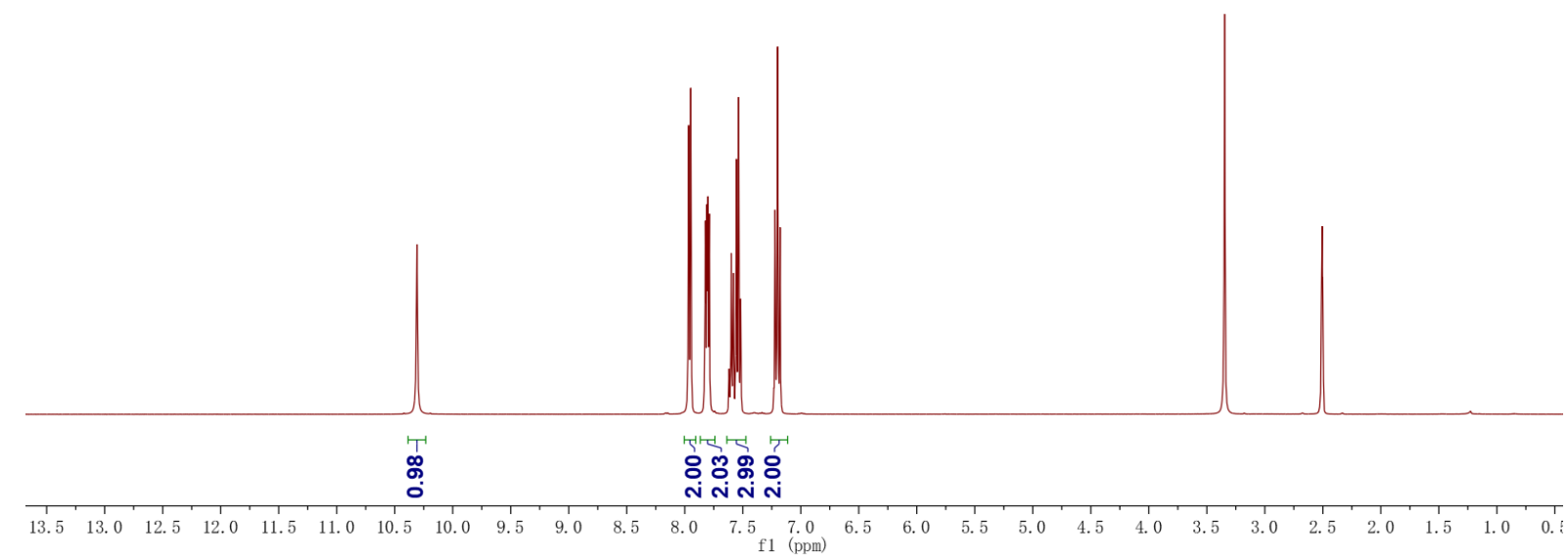

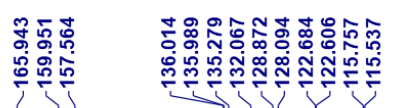

${ }^{13} \mathrm{C}$ NMR $\left(400 \mathrm{MHz}, \mathrm{DMSO}-d_{6}\right)$

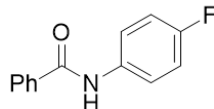

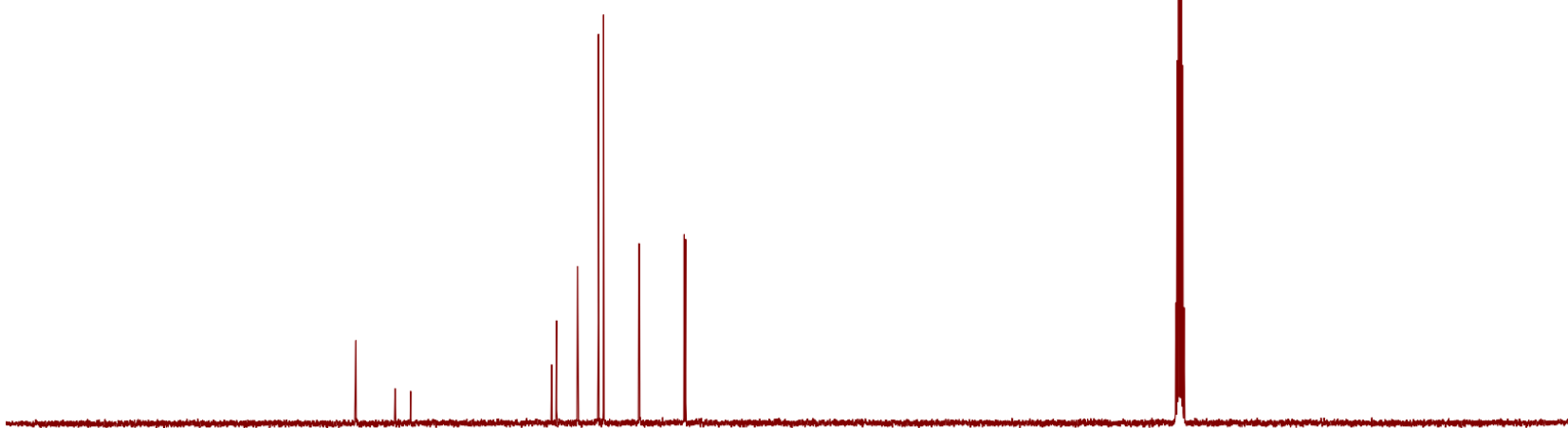

$\begin{array}{llllllllllll}210 & 200 & 190 & 180 & 170 & 160 & 150 & 140 & 130 & 120 & 110 & \begin{array}{c}100 \\ \mathrm{f} 1(\mathrm{ppm})\end{array}\end{array}$

Figure S24. ${ }^{1} \mathrm{H}$ NMR and ${ }^{13} \mathrm{C}\left\{{ }^{1} \mathrm{H}\right\}$ NMR Spectra of $N$-(4-fluorophenyl)benzamide (4b). 
算

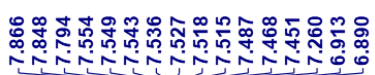

${ }^{1} \mathrm{H} \mathrm{NMR}\left(400 \mathrm{MHz}, \mathrm{CDCl}_{3}\right)$

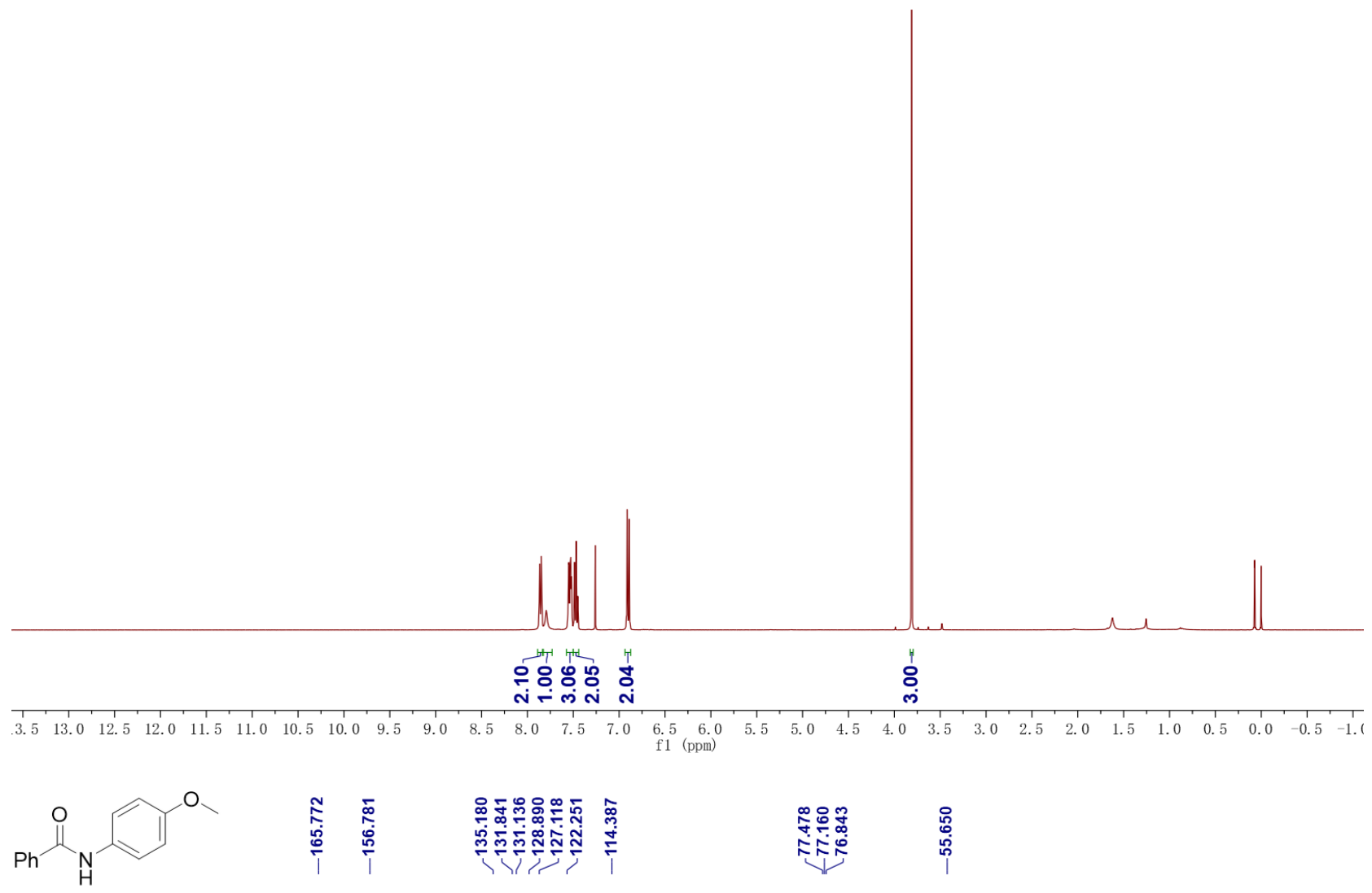

${ }^{13} \mathrm{C} \mathrm{NMR}\left(100 \mathrm{MHz}, \mathrm{CDCl}_{3}\right)$

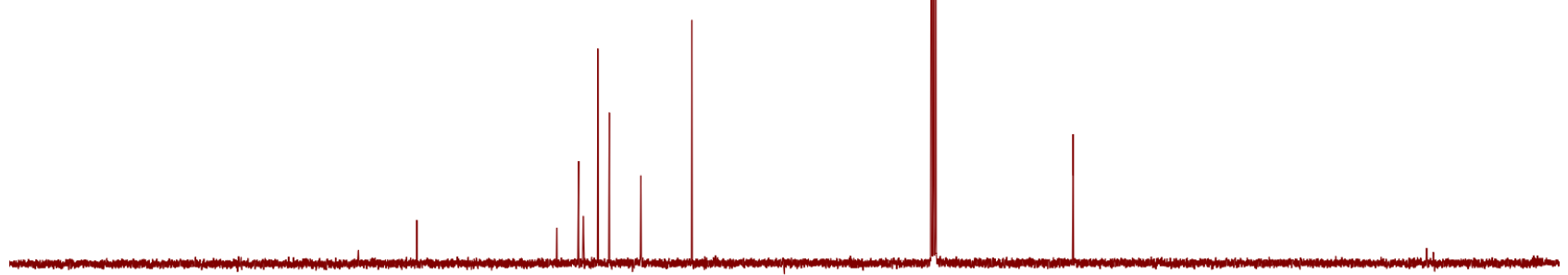

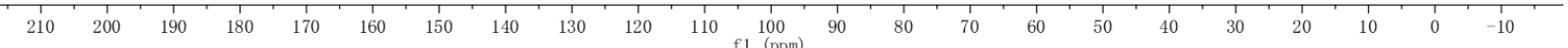

Figure S25. ${ }^{1} \mathrm{H}$ NMR and $\left.{ }^{13} \mathrm{C}\left\{{ }^{1} \mathrm{H}\right\}\right\}$ NMR Spectra of $N$-(p-methoxyphenyl)benzamide (4c). 

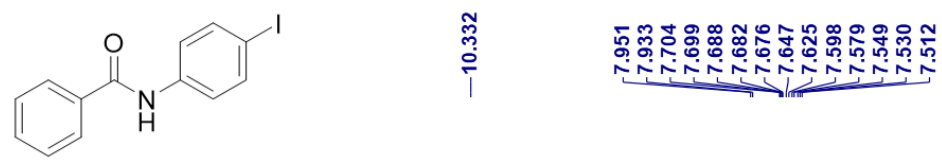

${ }^{1} \mathrm{H}$ NMR (400 MHz, DMSO- $\left.d_{6}\right)$
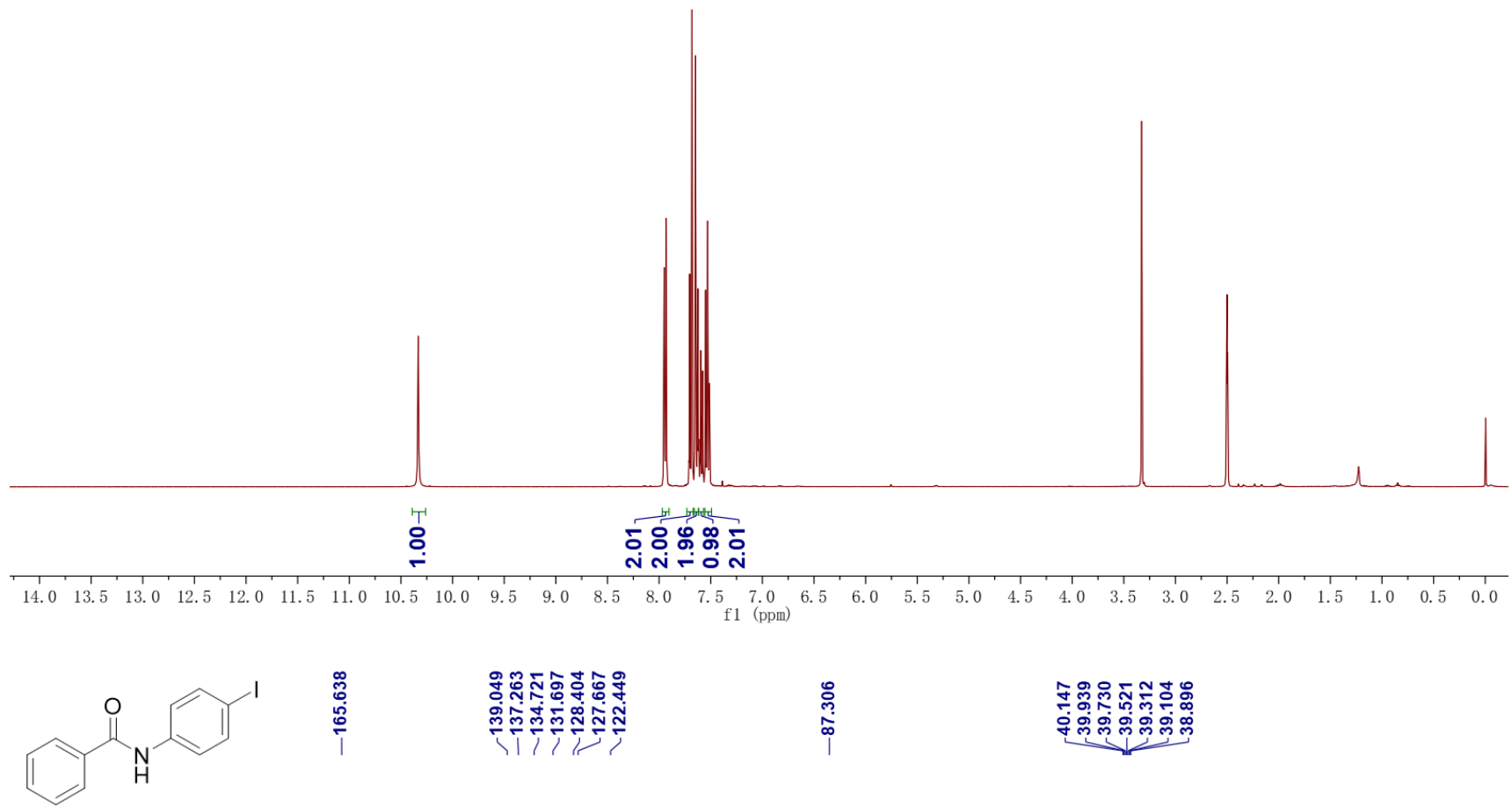

${ }^{13} \mathrm{C}$ NMR (400 MHz, DMSO- $\left.d_{6}\right)$

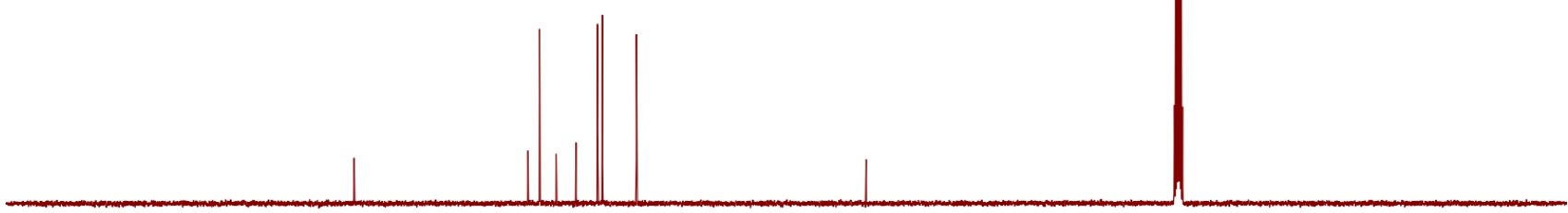

$\begin{array}{llllllllllll}210 & 200 & 190 & 180 & 170 & 160 & 150 & 140 & 130 & 120 & 110 & 100\end{array}$

Figure S26. ${ }^{1} \mathrm{H}$ NMR and ${ }^{13} \mathrm{C}\left\{{ }^{1} \mathrm{H}\right\}$ NMR Spectra of $N$-(4-iodophenyl)benzamide (4d). 


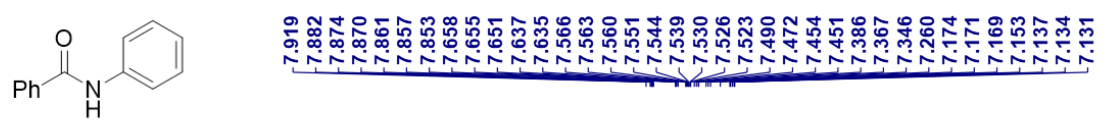

${ }^{1} \mathrm{H} \mathrm{NMR}\left(400 \mathrm{MHz}, \mathrm{CDCl}_{3}\right)$

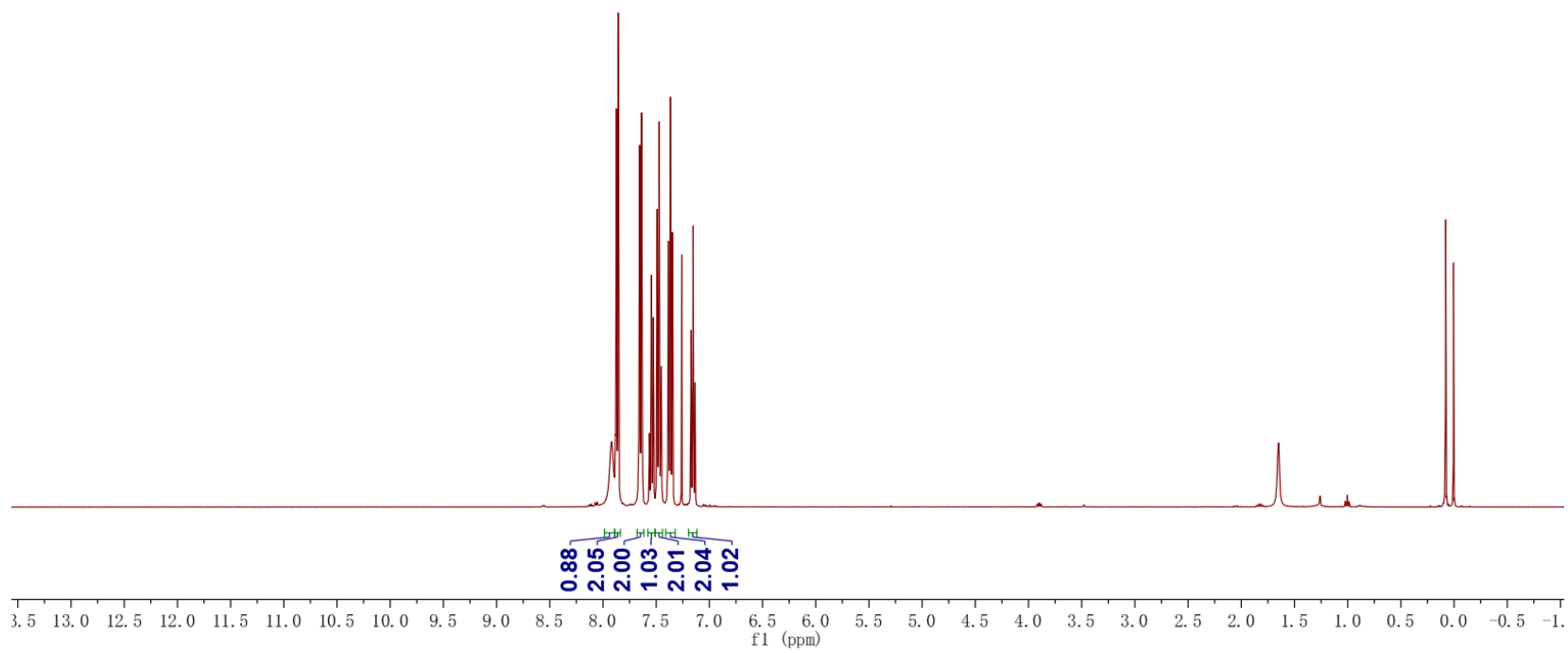

Ph

${ }^{13} \mathrm{C}$ NMR $\left(100 \mathrm{MHz}, \mathrm{CDCl}_{3}\right)$

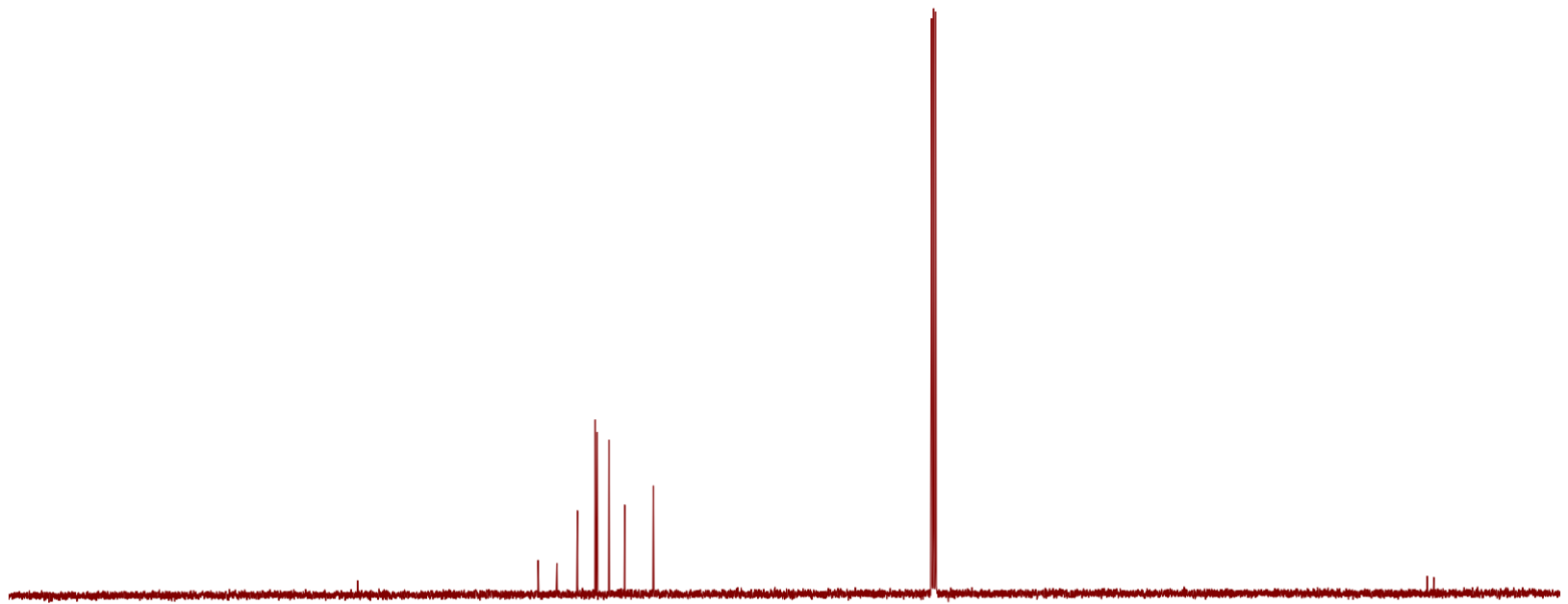

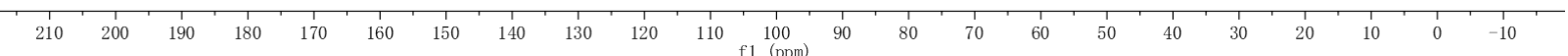

Figure S27. ${ }^{1} \mathrm{H}$ NMR and ${ }^{13} \mathrm{C}\left\{{ }^{1} \mathrm{H}\right\}$ NMR Spectra of $N$-phenylbenzamide (4e). 


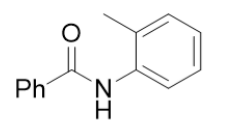

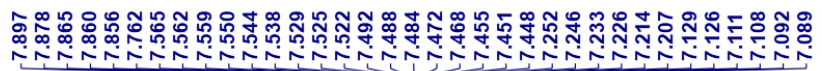

${ }^{1} \mathrm{H}$ NMR (400 MHz, $\mathrm{CDCl}_{3}$ )

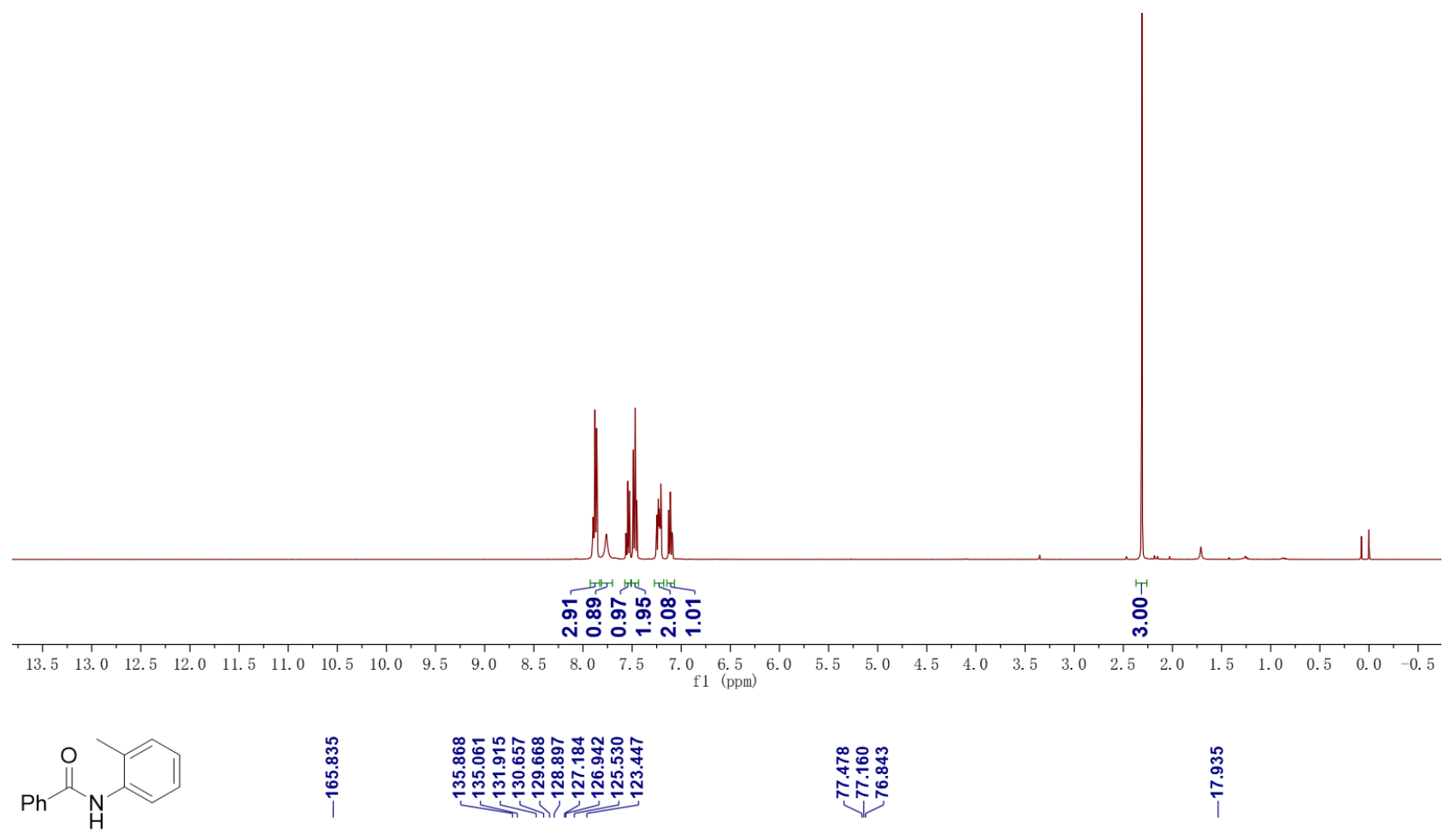

${ }^{13} \mathrm{C} \mathrm{NMR}\left(100 \mathrm{MHz}, \mathrm{CDCl}_{3}\right)$

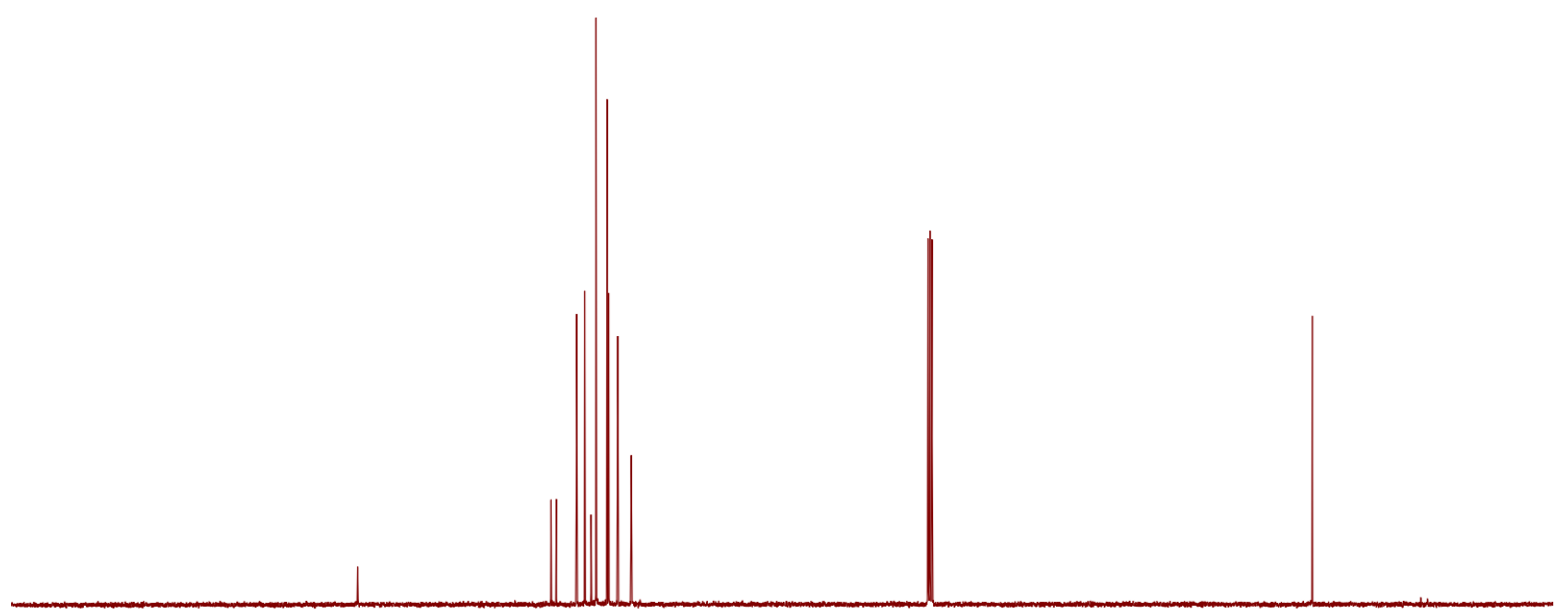

Figure S28. ${ }^{1} \mathrm{H}$ NMR and ${ }^{13} \mathrm{C}\left\{{ }^{1} \mathrm{H}\right\}$ NMR Spectra of $N$-benzoyl-2-methylaniline (4f). 


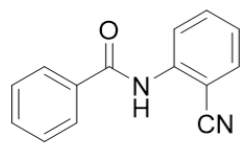

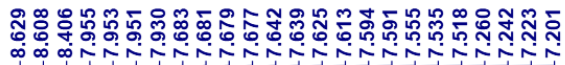

${ }^{1} \mathrm{H} \mathrm{NMR}\left(400 \mathrm{MHz}, \mathrm{CDCl}_{3}\right)$

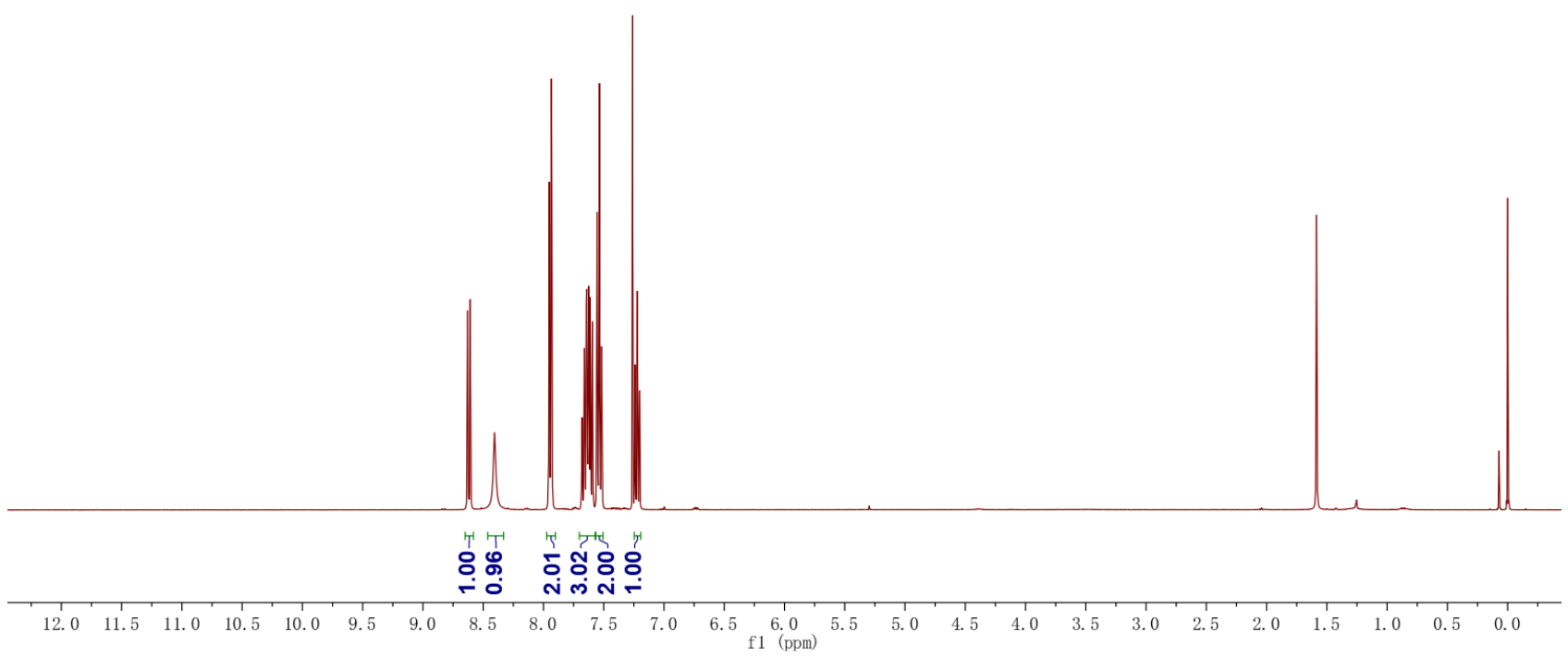<smiles>N#Cc1ccccc1NC(=O)c1ccccc1</smiles>

每

${ }^{13} \mathrm{C}$ NMR $\left(100 \mathrm{MHz}, \mathrm{CDCl}_{3}\right)$

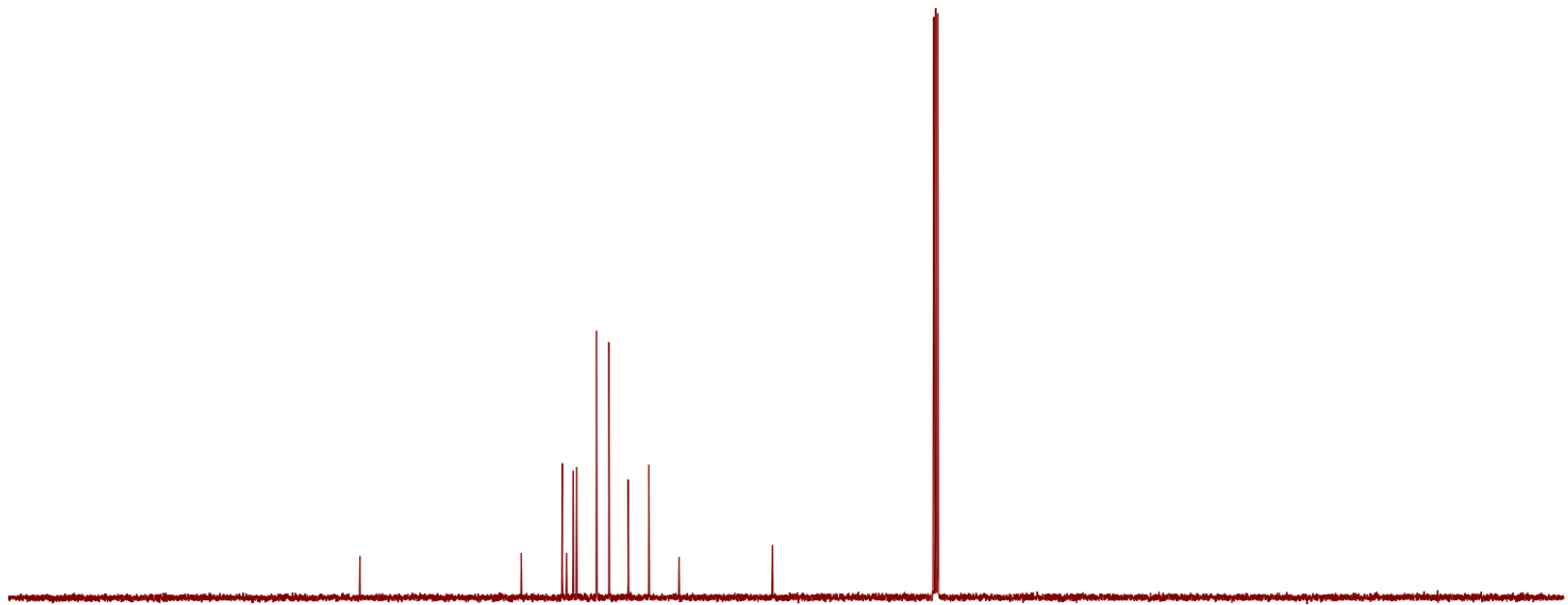

$\begin{array}{lrrrrrrrrrrr}210 & 200 & 190 & 180 & 170 & 160 & 150 & 140 & 130 & 120 & 110 & 100\end{array}$

Figure S29. ${ }^{1} \mathrm{H}$ NMR and ${ }^{13} \mathrm{C}\left\{{ }^{1} \mathrm{H}\right\}$ NMR Spectra of $N$-(2-cyanophenyl)benzamide (4g). 
$\overbrace{\mathrm{NO}}^{\mathrm{N}}$

${ }^{1} \mathrm{H}$ NMR (400 MHz, $\mathrm{CDCl}_{3}$ )

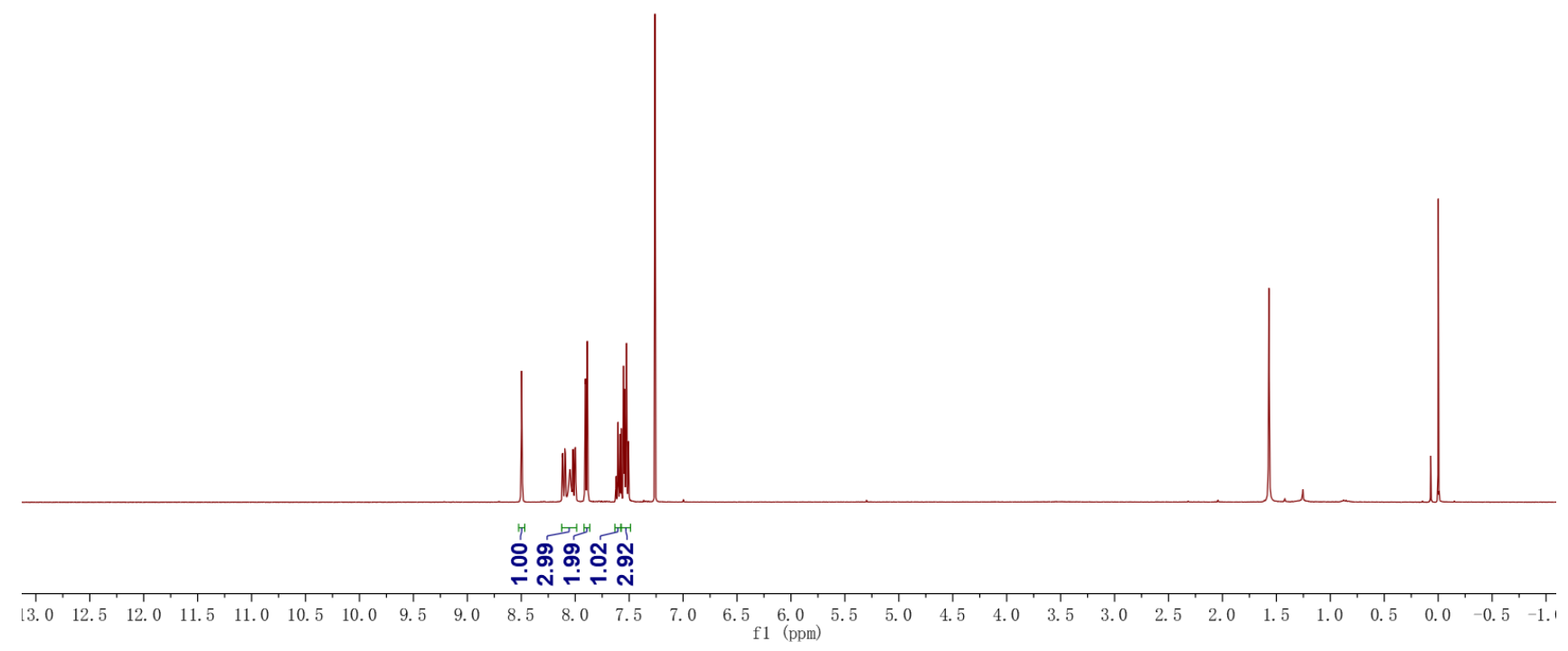

${ }^{13} \mathrm{C}$ NMR (100 MHz, $\left.\mathrm{CDCl}_{3}\right)$

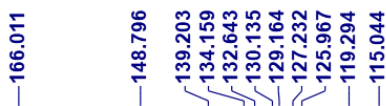


$\operatorname{Ph}^{\mathrm{O}} \stackrel{\text { H }}{\mathrm{O}}$

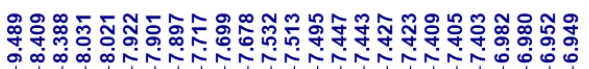

${ }^{1} \mathrm{H} \mathrm{NMR}\left(400 \mathrm{MHz}, \mathrm{CDCl}_{3}\right)$

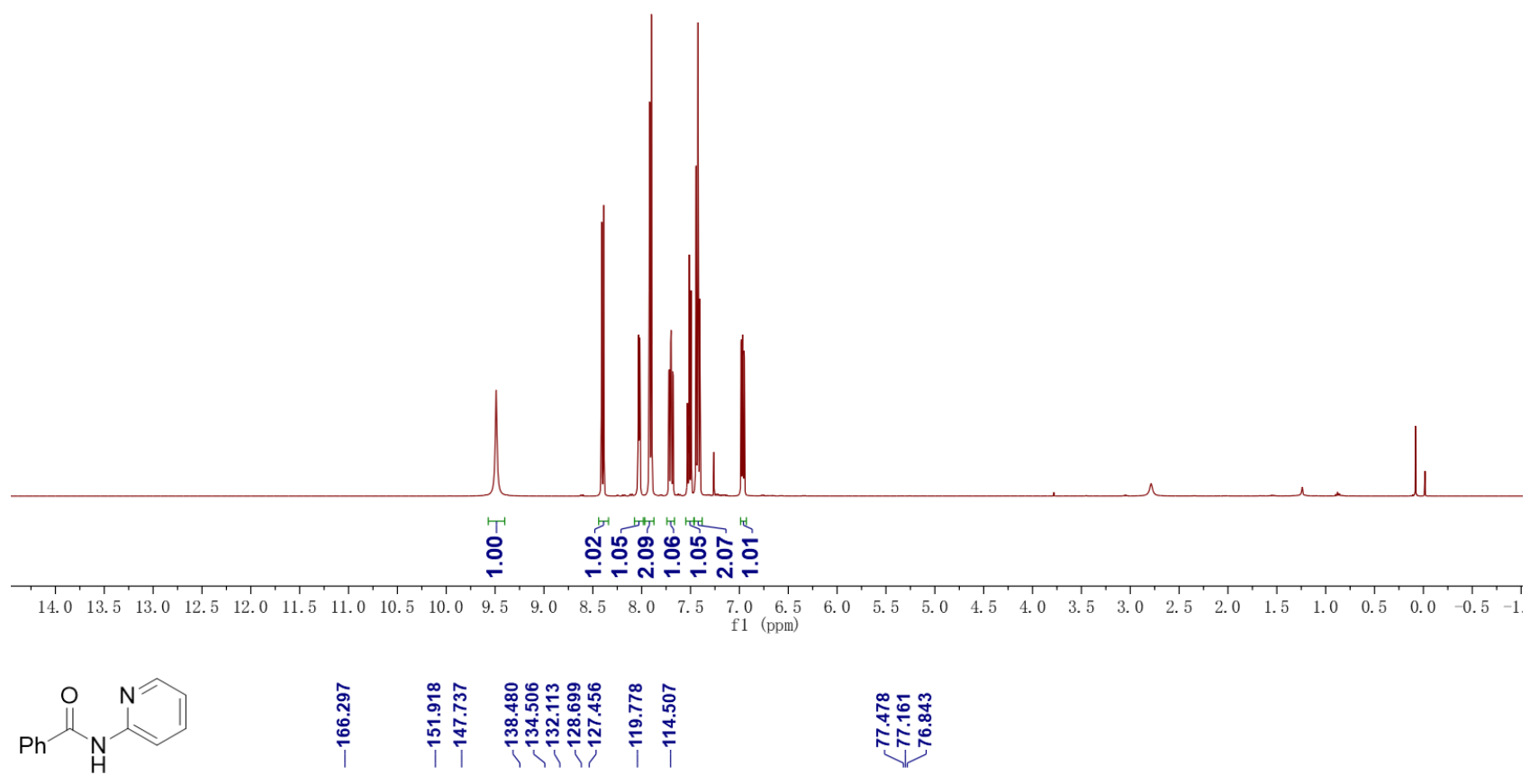

${ }^{13} \mathrm{C}$ NMR $\left(100 \mathrm{MHz}, \mathrm{CDCl}_{3}\right)$

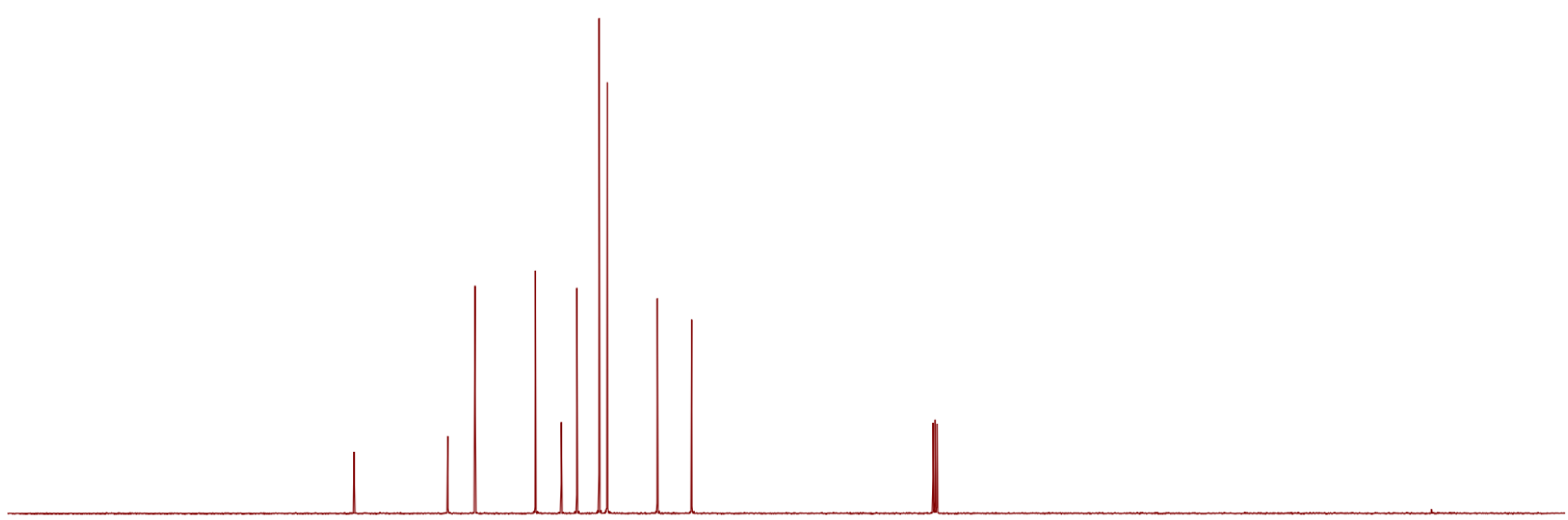

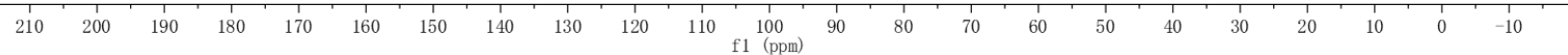

Figure S31. ${ }^{1} \mathrm{H}$ NMR and ${ }^{13} \mathrm{C}\left\{{ }^{1} \mathrm{H}\right\}$ NMR Spectra of $N$-(pyridin-2-yl)benzamide (4i). 
 웅ำ

${ }^{1} \mathrm{H}$ NMR (400 MHz, DMSO-d $\left.d_{6}\right)$

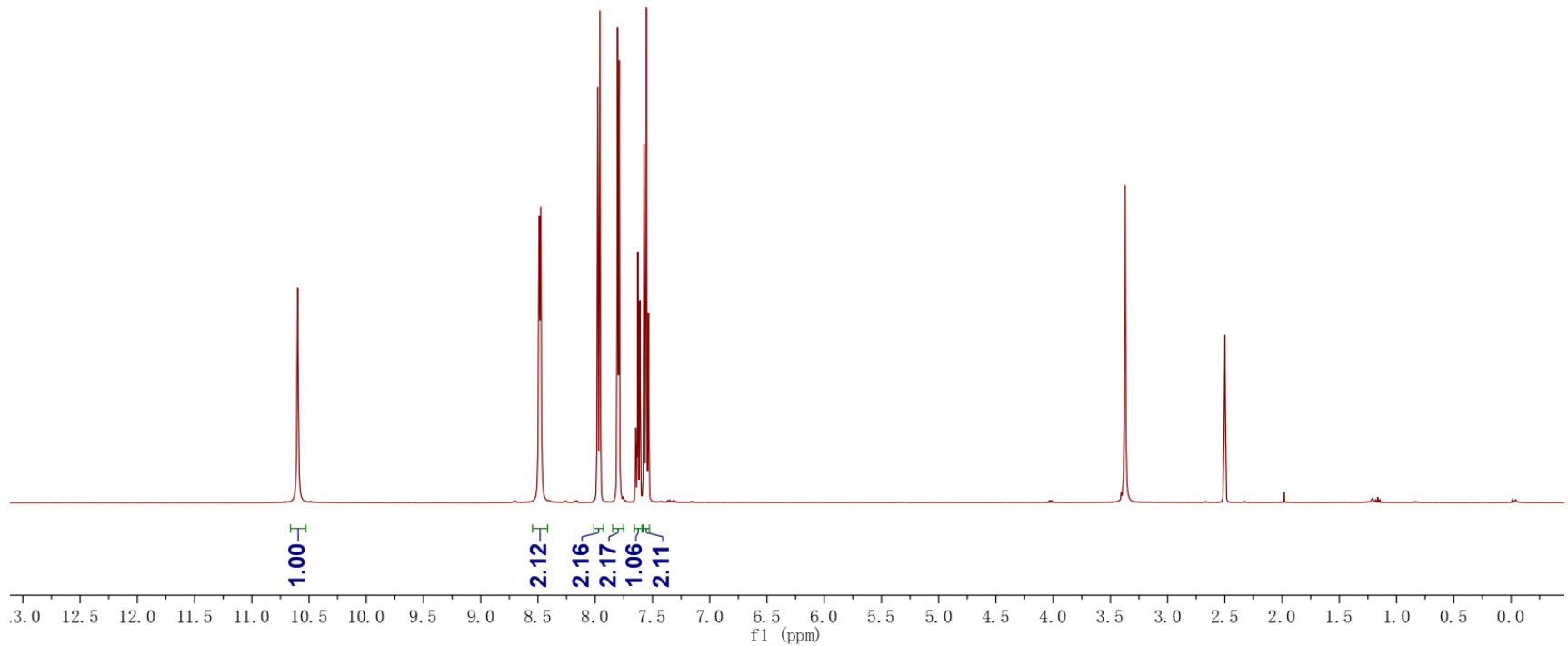

Ph $\mathrm{N}_{\mathrm{H}}^{\mathrm{O}}$ 少

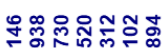

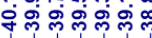

${ }^{13} \mathrm{C}$ NMR (400 MHz, DMSO- $d_{6}$ )

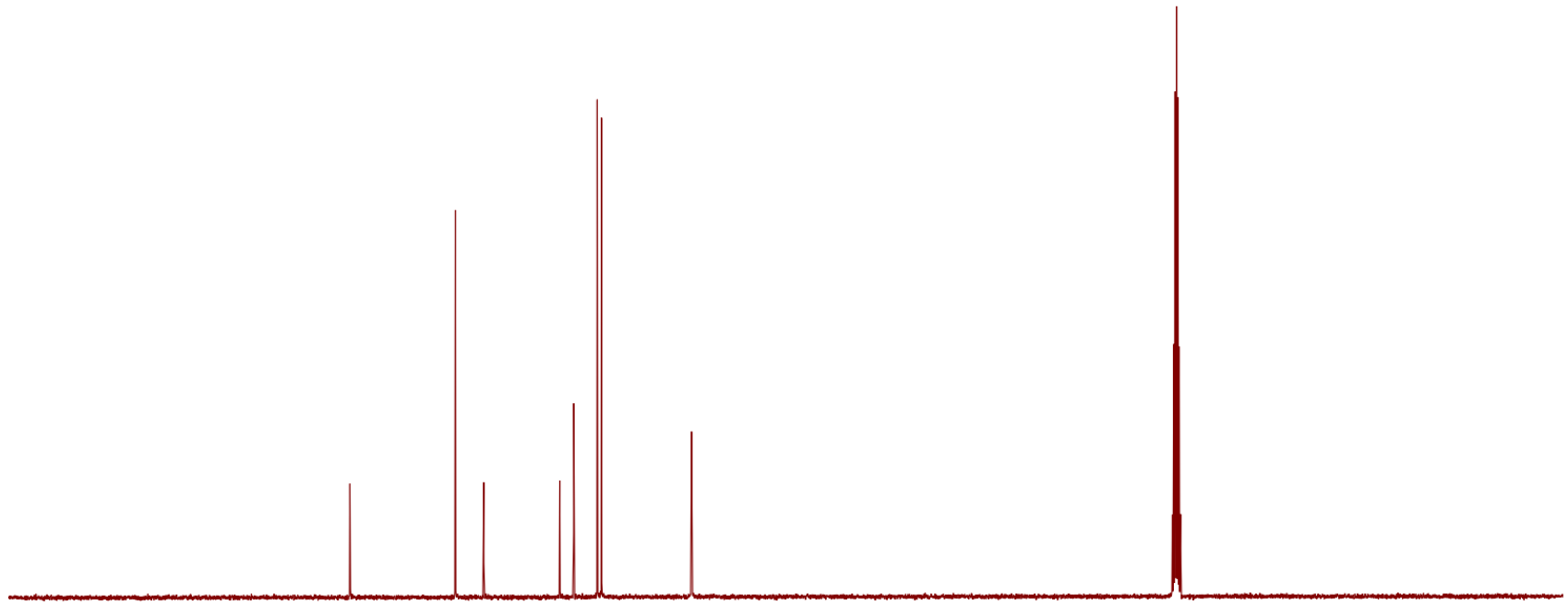

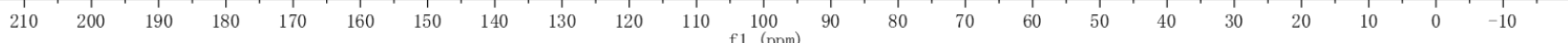

Figure S32. ${ }^{1} \mathrm{H}$ NMR and ${ }^{13} \mathrm{C}\left\{{ }^{1} \mathrm{H}\right\}$ NMR Spectra of $N$-(pyridin-4-yl)benzamide (4j). 
算

${ }^{1} \mathrm{H} \mathrm{NMR}\left(400 \mathrm{MHz}, \mathrm{CDCl}_{3}\right)$

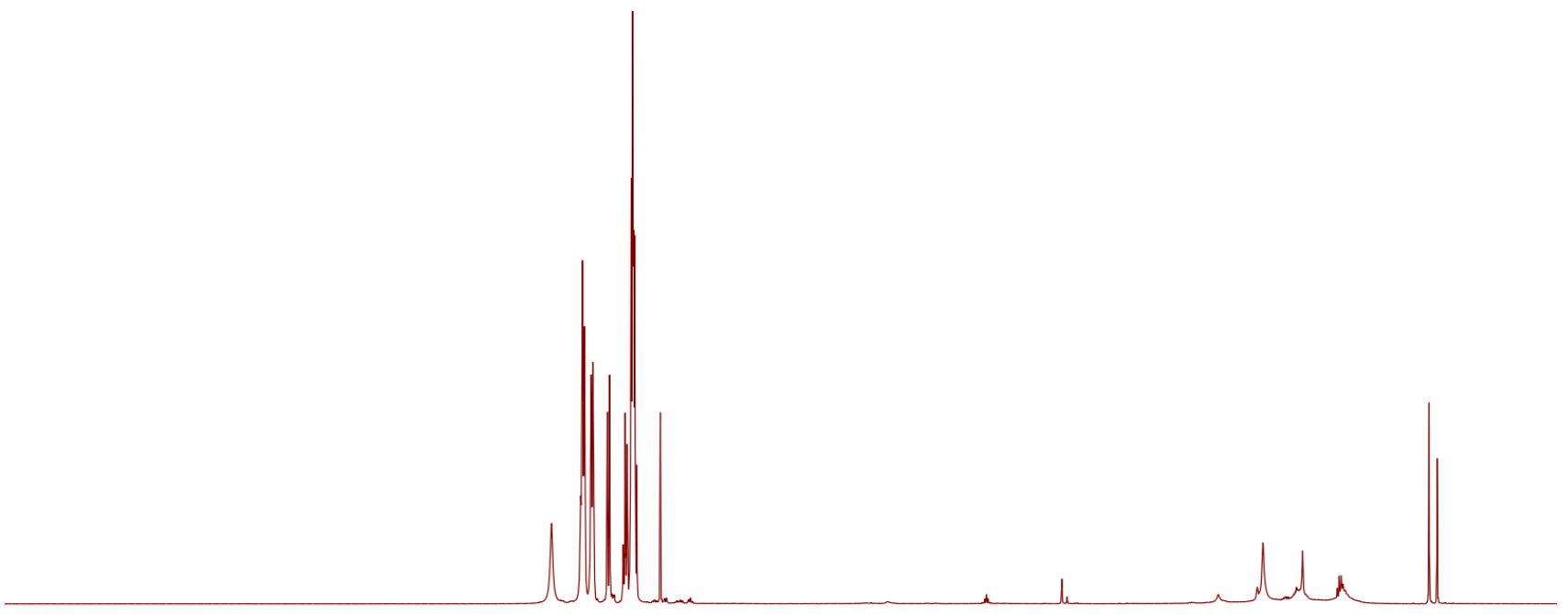

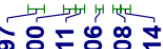

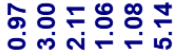

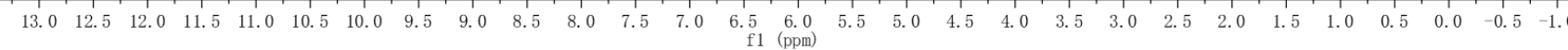
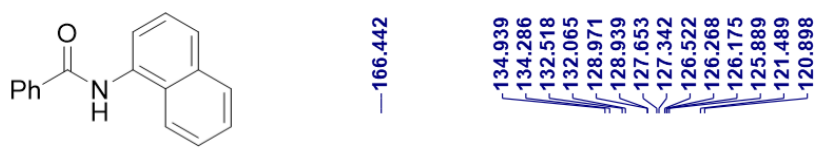

${ }^{13} \mathrm{C}$ NMR $\left(100 \mathrm{MHz}, \mathrm{CDCl}_{3}\right)$
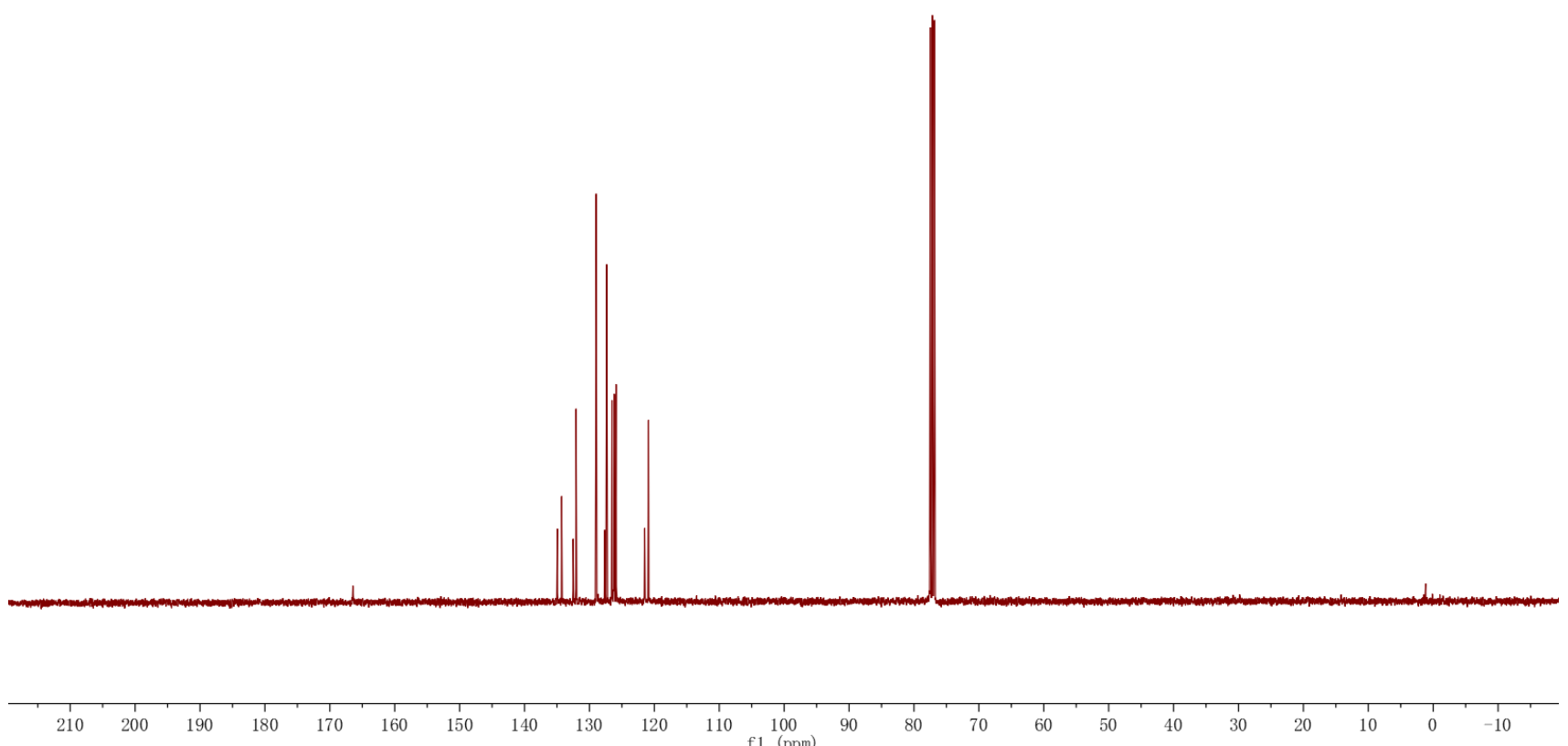

Figure S33. ${ }^{1} \mathrm{H}$ NMR and ${ }^{13} \mathrm{C}\left\{{ }^{1} \mathrm{H}\right\}$ NMR Spectra of $N$-1-naphthalenyl-benzamide (4k). 
Ph

${ }^{1} \mathrm{H}$ NMR $\left(400 \mathrm{MHz}, \mathrm{CDCl}_{3}\right)$

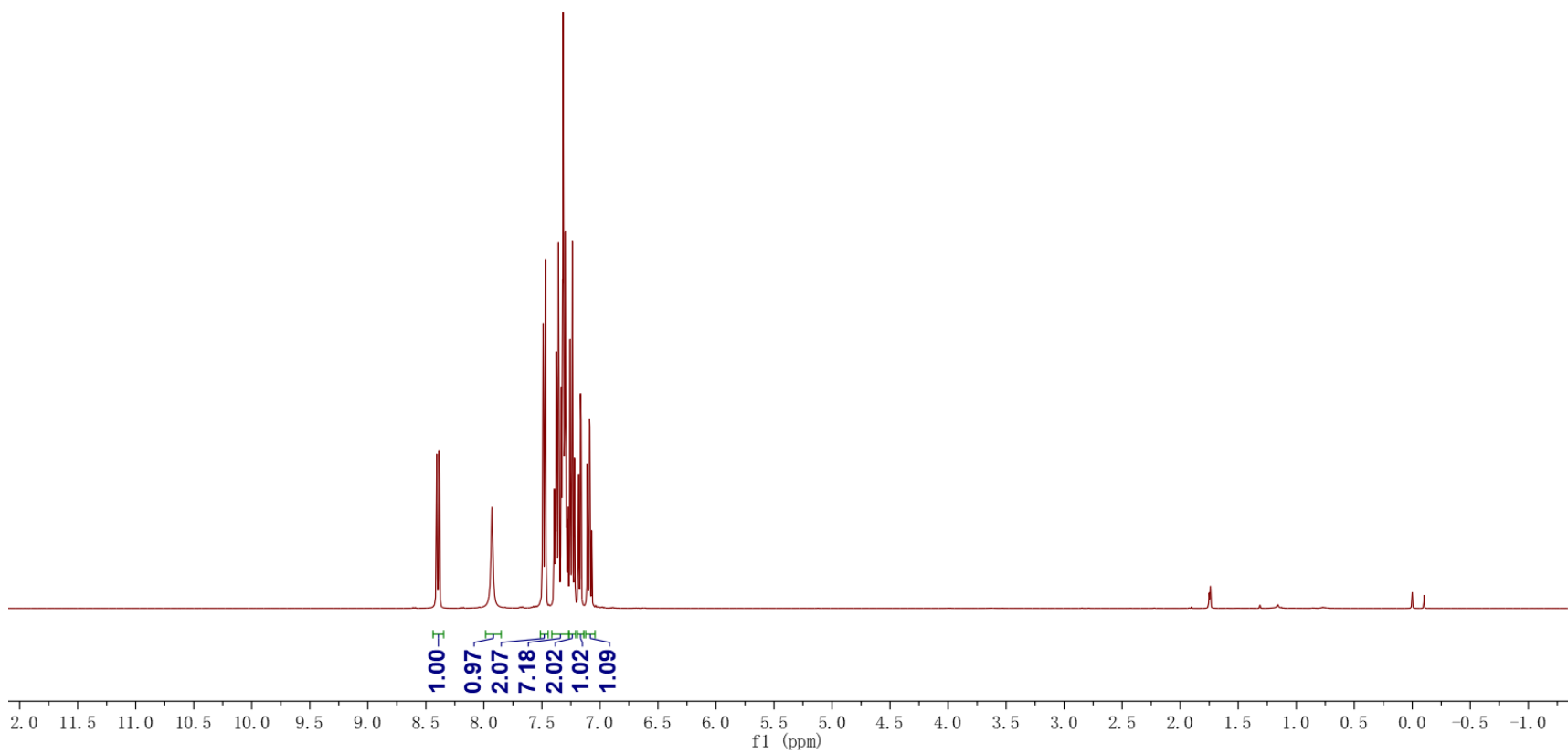

算

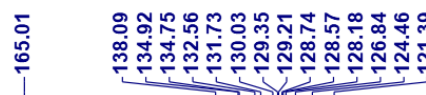

尖少

${ }^{13} \mathrm{C} \mathrm{NMR}\left(100 \mathrm{MHz}, \mathrm{CDCl}_{3}\right)$

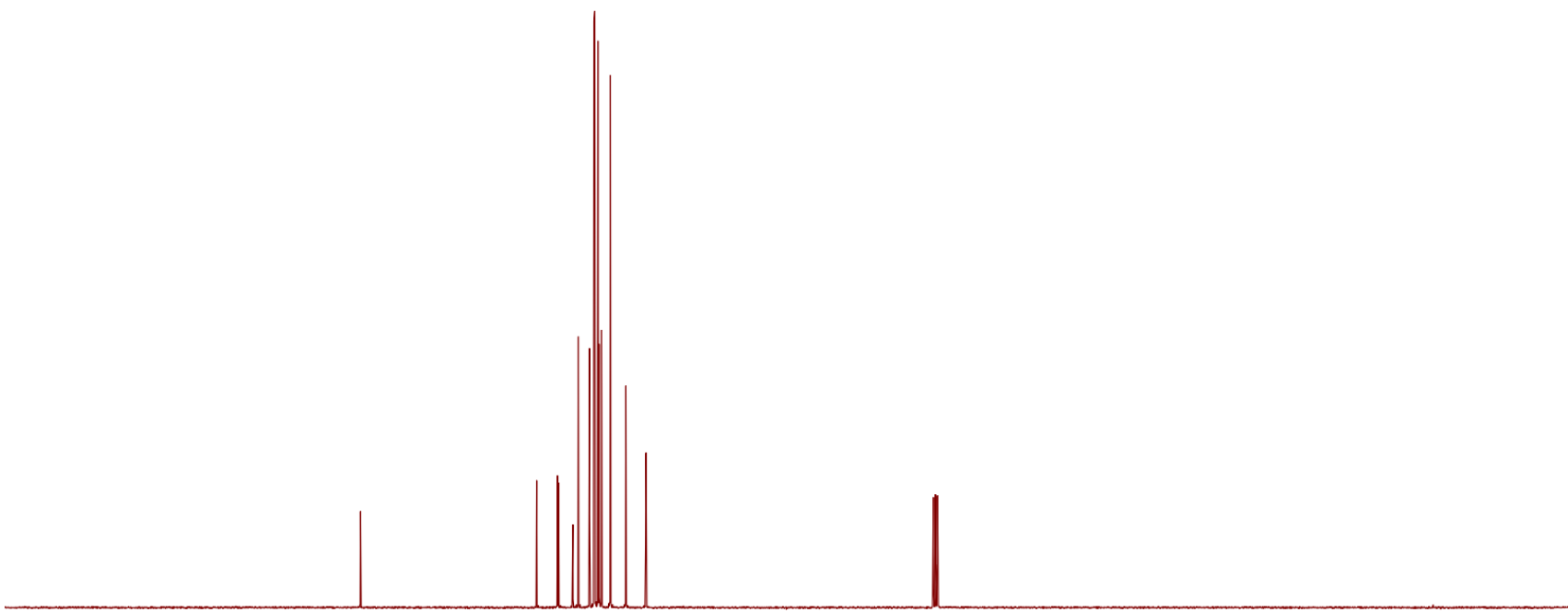

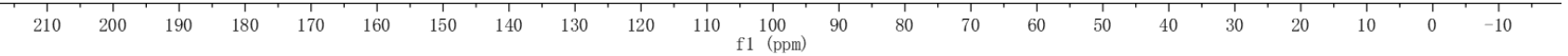

Figure S34. ${ }^{1} \mathrm{H}$ NMR and ${ }^{13} \mathrm{C}\left\{{ }^{1} \mathrm{H}\right\}$ NMR Spectra of $N$-([1,1'-biphenyl]-2-yl)benzamide (4l). 

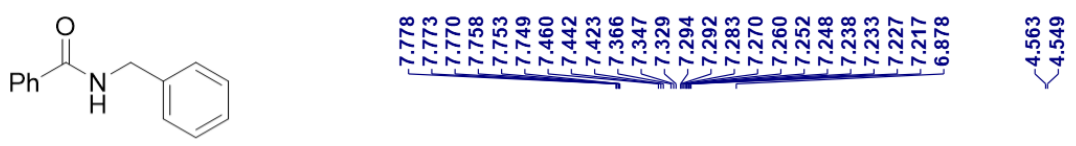

${ }^{1} \mathrm{H}$ NMR $\left(400 \mathrm{MHz}, \mathrm{CDCl}_{3}\right)$

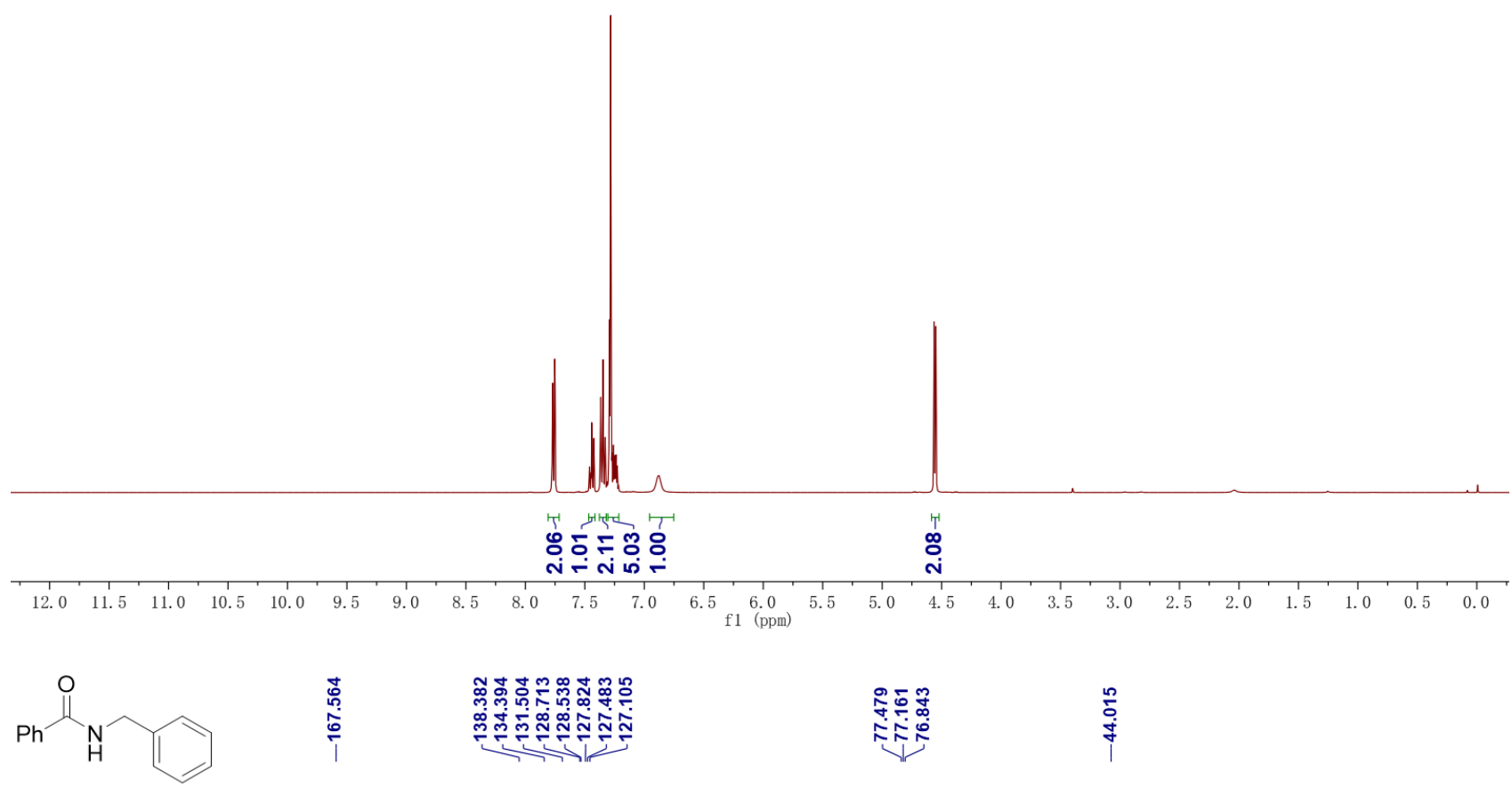

${ }^{13} \mathrm{C} \mathrm{NMR}\left(100 \mathrm{MHz}, \mathrm{CDCl}_{3}\right)$
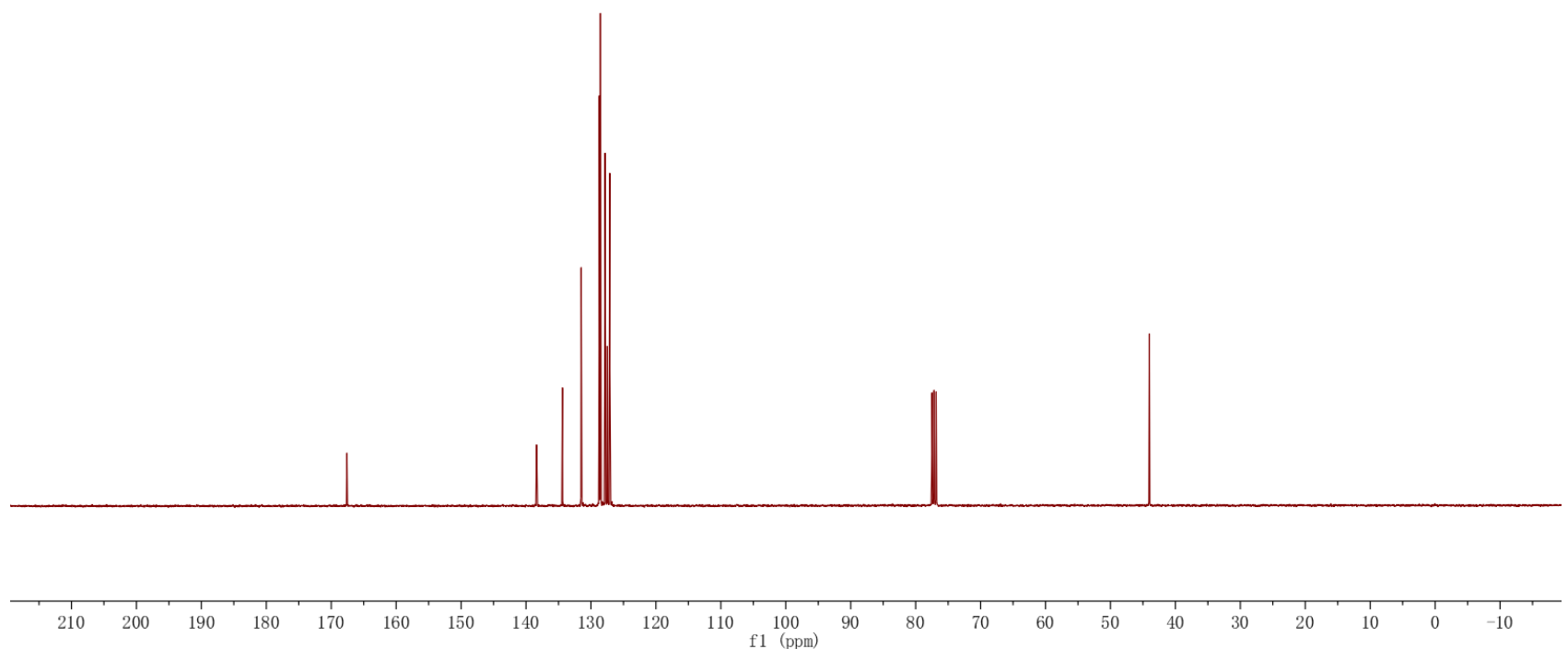

Figure S35. ${ }^{1} \mathrm{H}$ NMR and ${ }^{13} \mathrm{C}\left\{{ }^{1} \mathrm{H}\right\}$ NMR Spectra of $N$-benzylbenzamide (4m). 
${ }_{\mathrm{ph}} \mathrm{P}_{\mathrm{H}} \mathrm{C}$

${ }^{1} \mathrm{H}$ NMR $\left(400 \mathrm{MHz}, \mathrm{CDCl}_{3}\right.$ )

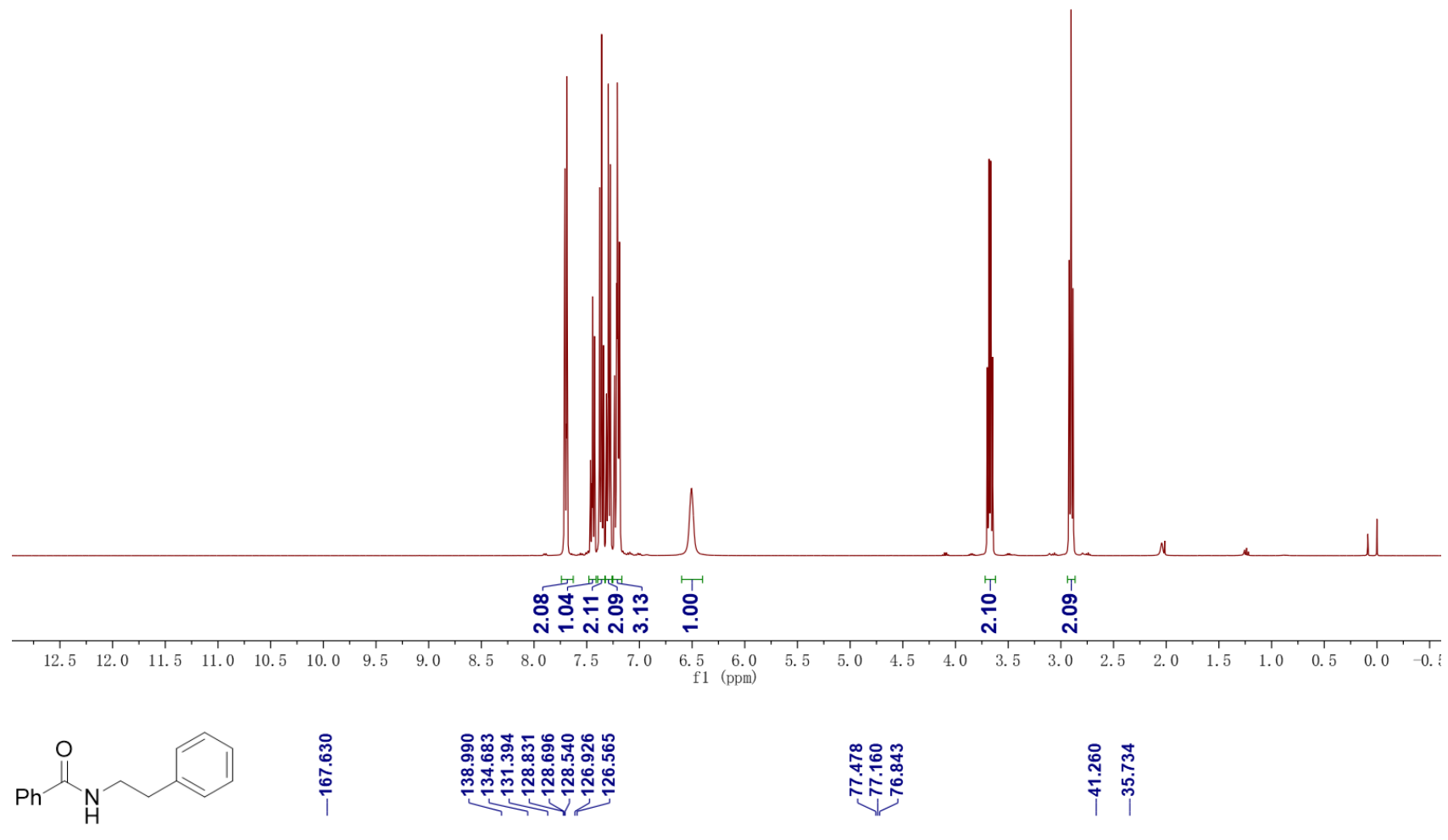

${ }^{13} \mathrm{C} \mathrm{NMR}\left(100 \mathrm{MHz}, \mathrm{CDCl}_{3}\right)$

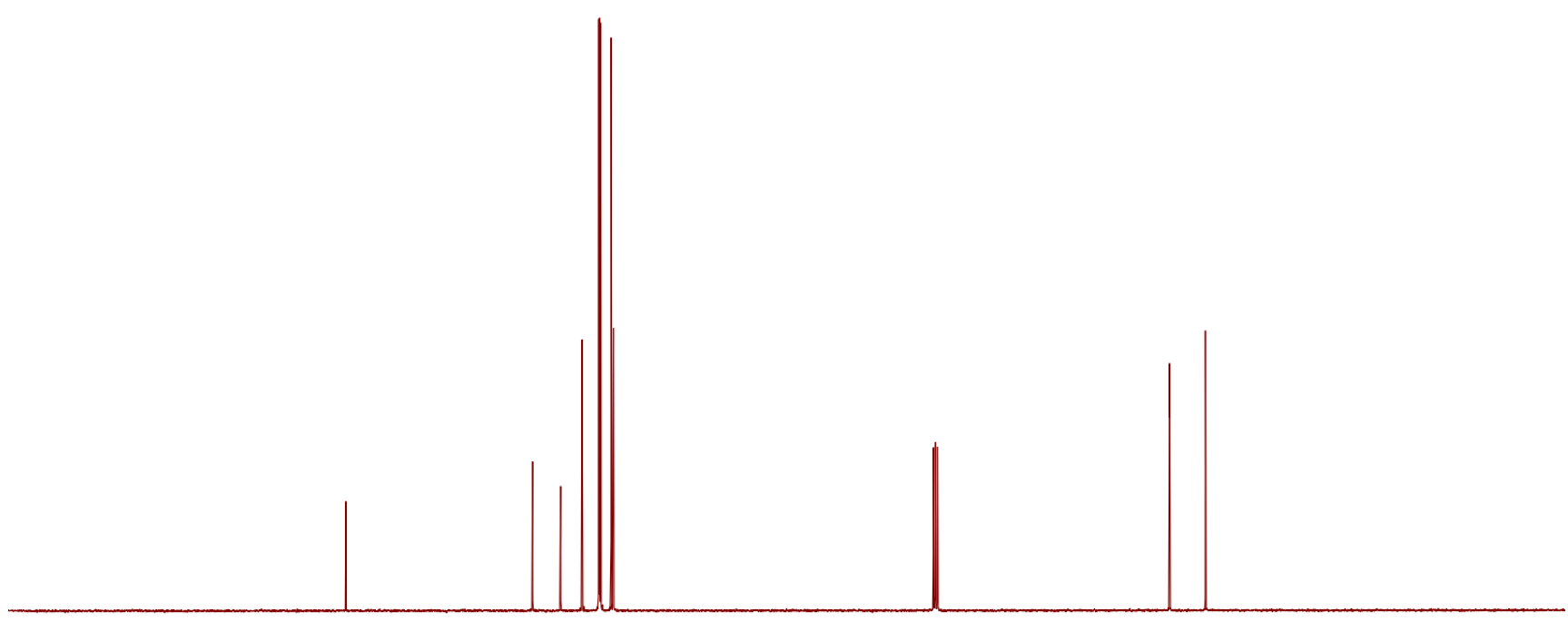

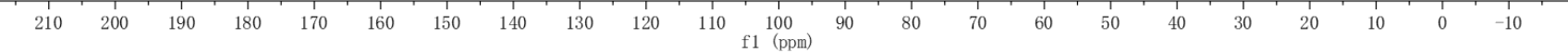

Figure S36. ${ }^{1} \mathrm{H}$ NMR and ${ }^{13} \mathrm{C}\left\{{ }^{1} \mathrm{H}\right\}$ NMR Spectra of $N$-phenethylbenzamide (4n). 
Ph

${ }^{1} \mathrm{H} \mathrm{NMR}\left(400 \mathrm{MHz}, \mathrm{CDCl}_{3}\right)$

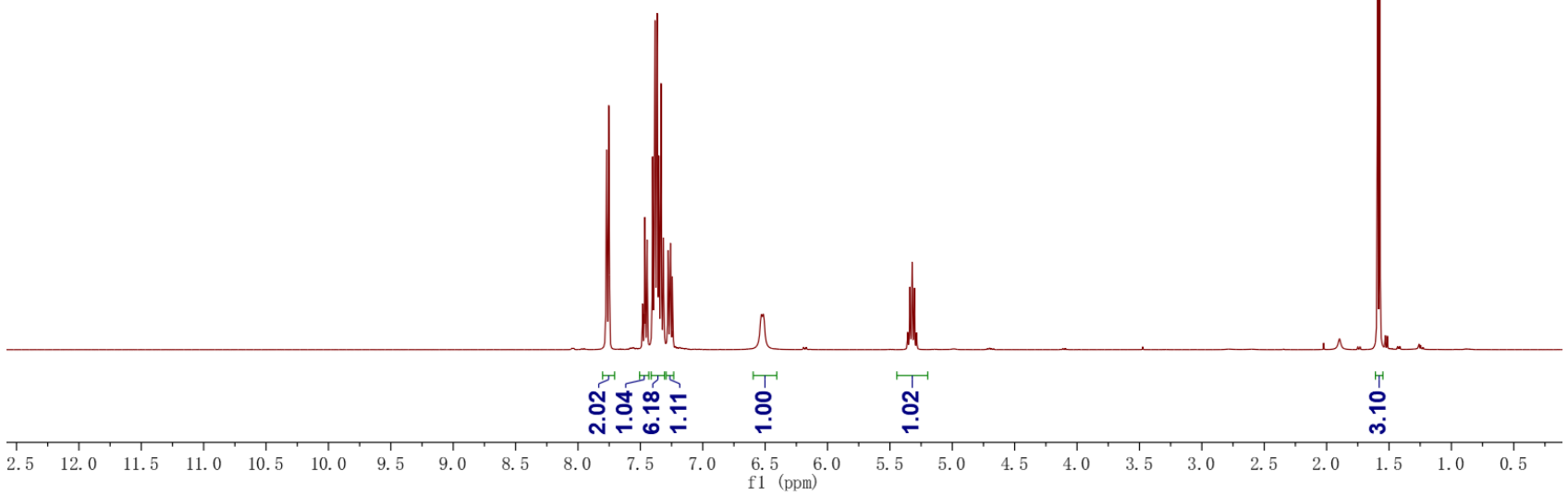<smiles>CC(NC(=O)c1ccccc1)c1ccccc1</smiles>

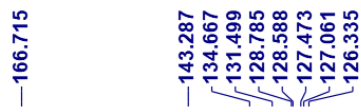

证篎

官

${ }^{13} \mathrm{C} \mathrm{NMR}\left(100 \mathrm{MHz}, \mathrm{CDCl}_{3}\right)$

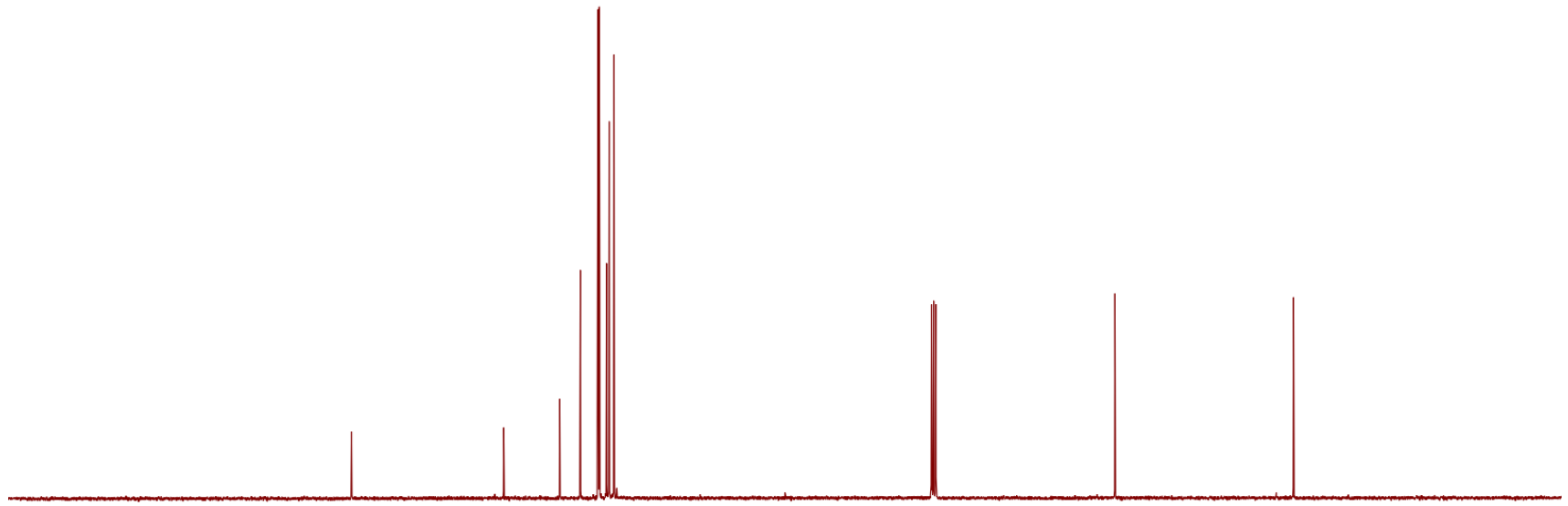

$\begin{array}{llllllllllll}210 & 200 & 190 & 180 & 170 & 160 & 150 & 140 & 130 & 120 & 110 & \begin{array}{l}100 \\ \mathrm{f} 1\end{array}(\mathrm{p})\end{array}$

Figure S37. ${ }^{1} \mathrm{H}$ NMR and ${ }^{13} \mathrm{C}\left\{{ }^{1} \mathrm{H}\right\}$ NMR Spectra of $N$-(1-phenylethyl)benzamide (4o). 
Ph

${ }^{1} \mathrm{H}$ NMR $\left(400 \mathrm{MHz}, \mathrm{CDCl}_{3}\right)$

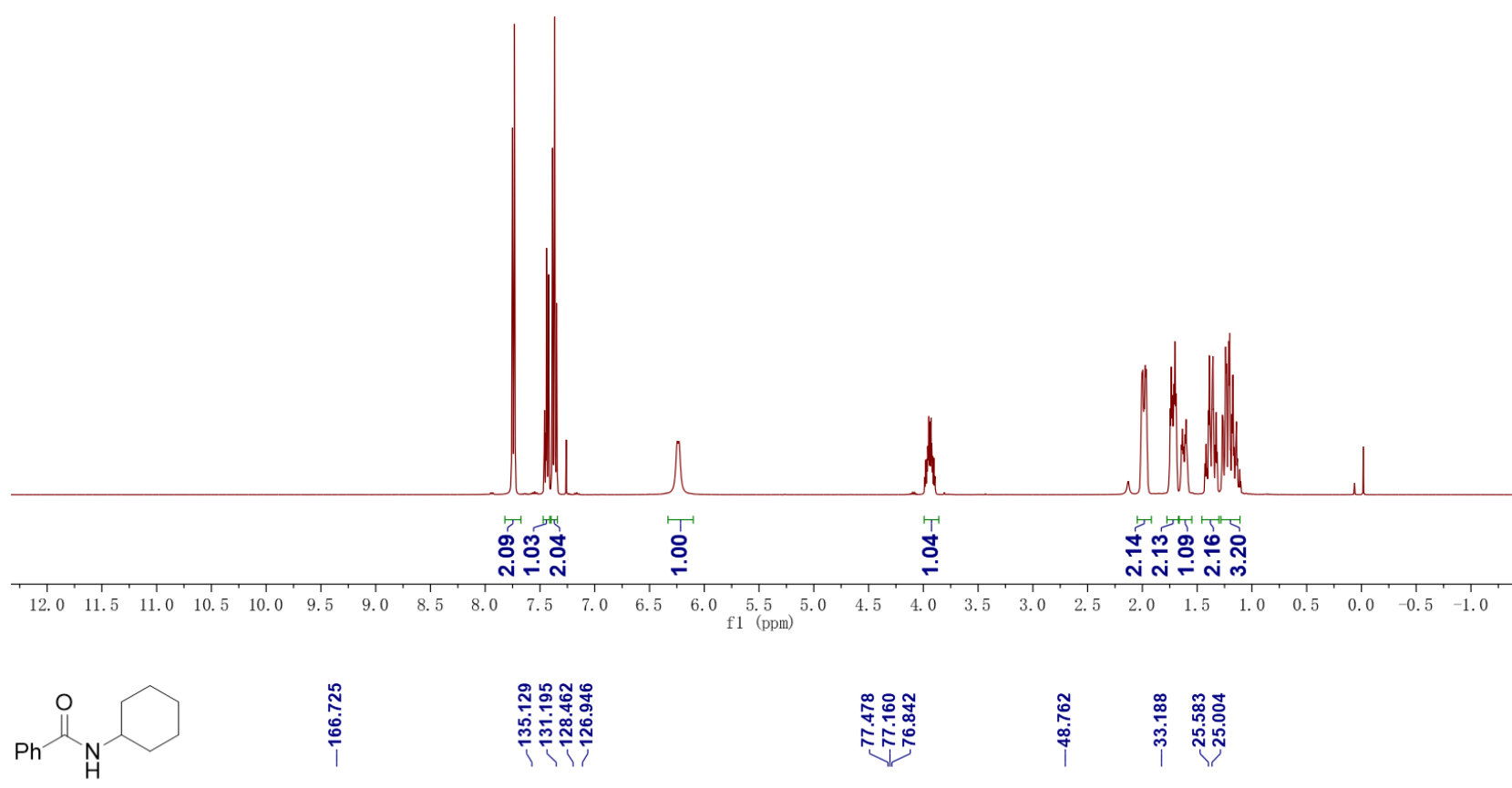

${ }^{13} \mathrm{C} \mathrm{NMR}\left(100 \mathrm{MHz}, \mathrm{CDCl}_{3}\right)$

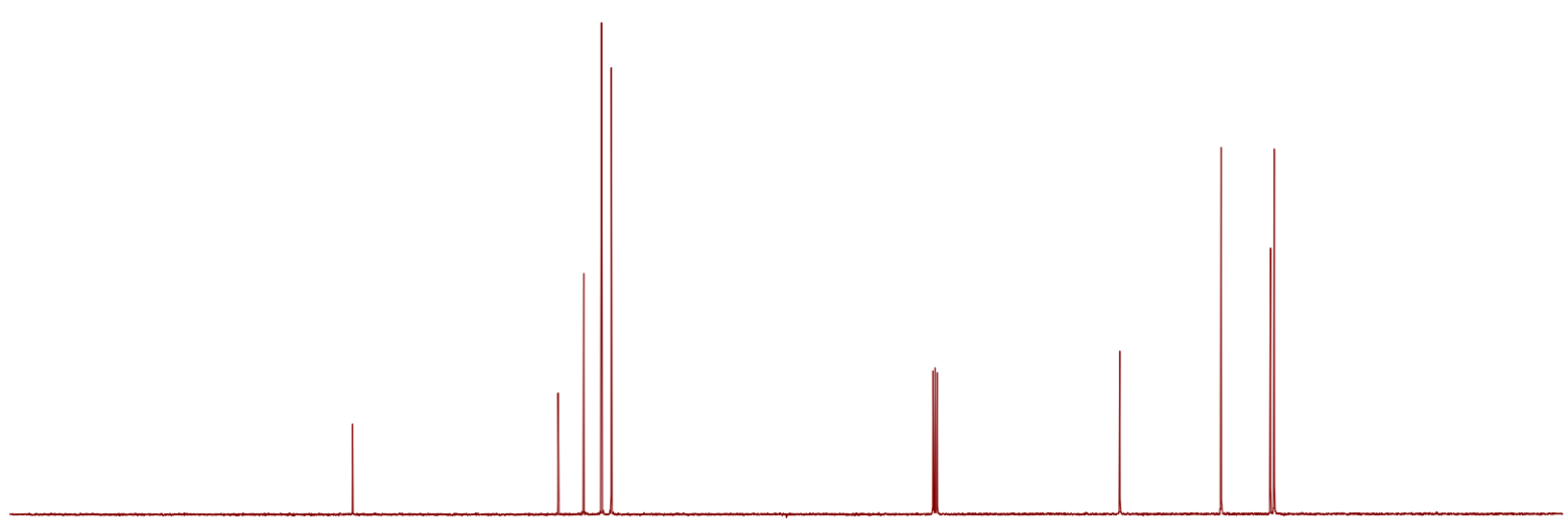

$\begin{array}{rrrrr}1 & 1 & 1 & 1 & 1 \\ 210 & 200 & 190 & 180 & 170\end{array}$

Figure S38. ${ }^{1} \mathrm{H}$ NMR and ${ }^{13} \mathrm{C}\left\{{ }^{1} \mathrm{H}\right\}$ NMR Spectra of $N$-cyclohexylbenzamide (4p). 


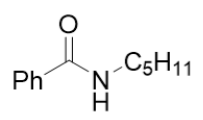

${ }^{1} \mathrm{H} \mathrm{NMR}\left(400 \mathrm{MHz}, \mathrm{CDCl}_{3}\right)$

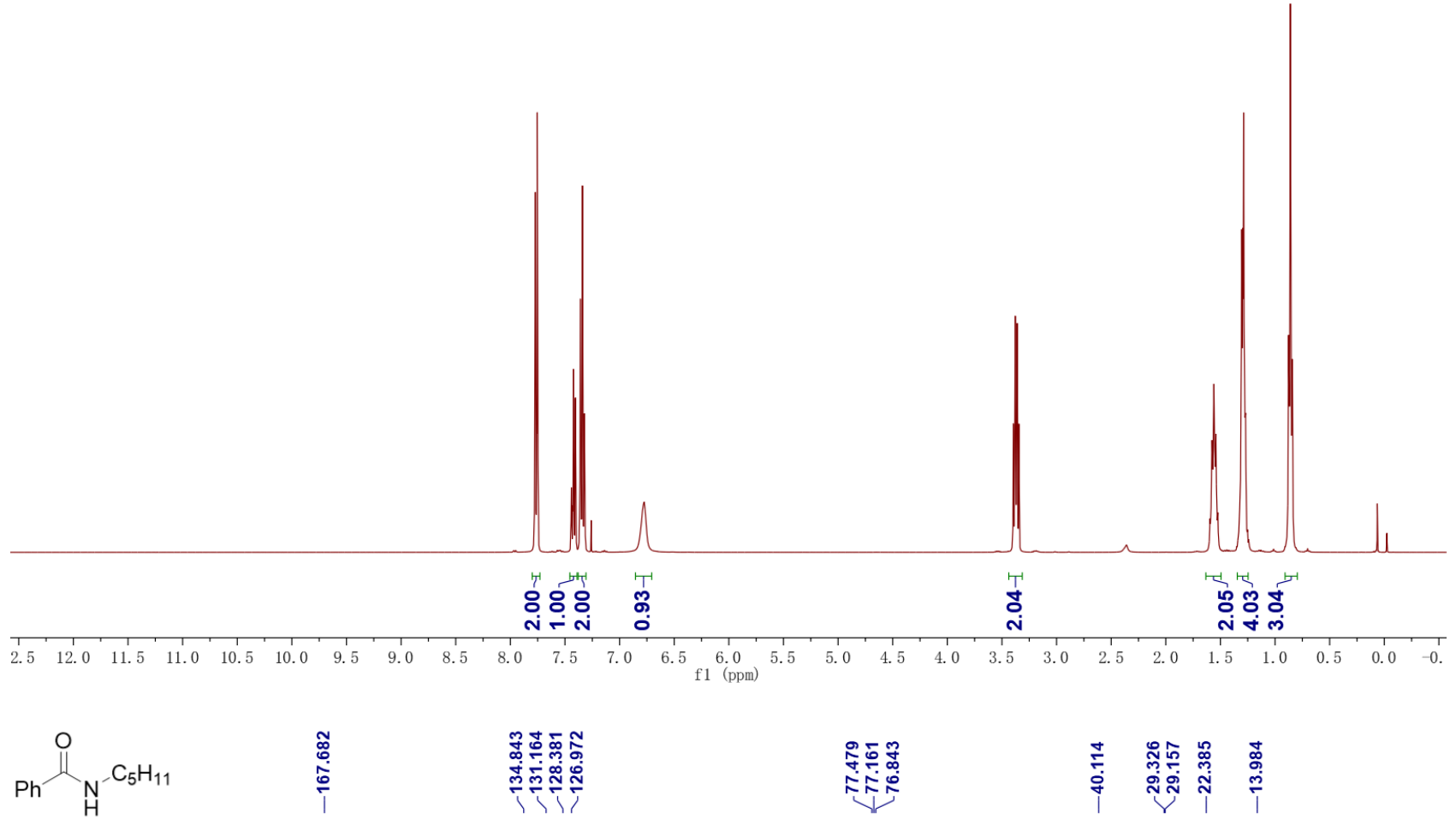

${ }^{13} \mathrm{C} \mathrm{NMR}\left(100 \mathrm{MHz}, \mathrm{CDCl}_{3}\right)$

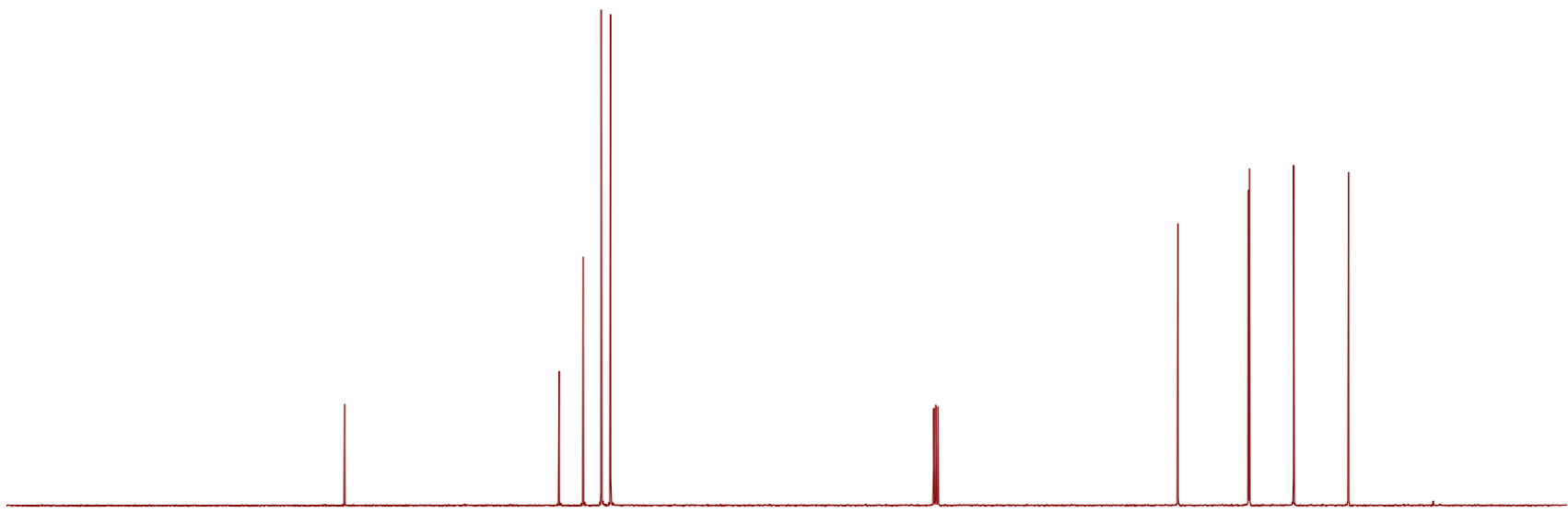

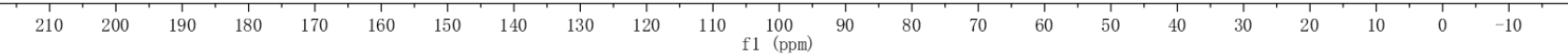

Figure S39. ${ }^{1} \mathrm{H}$ NMR and ${ }^{13} \mathrm{C}\left\{{ }^{1} \mathrm{H}\right\}$ NMR Spectra of $N$-pentylbenzamide (4q). 
<smiles>CCN1CCCC1CNC(=O)c1ccccc1</smiles>

${ }^{1} \mathrm{H}$ NMR $\left(400 \mathrm{MHz}, \mathrm{CDCl}_{3}\right)$

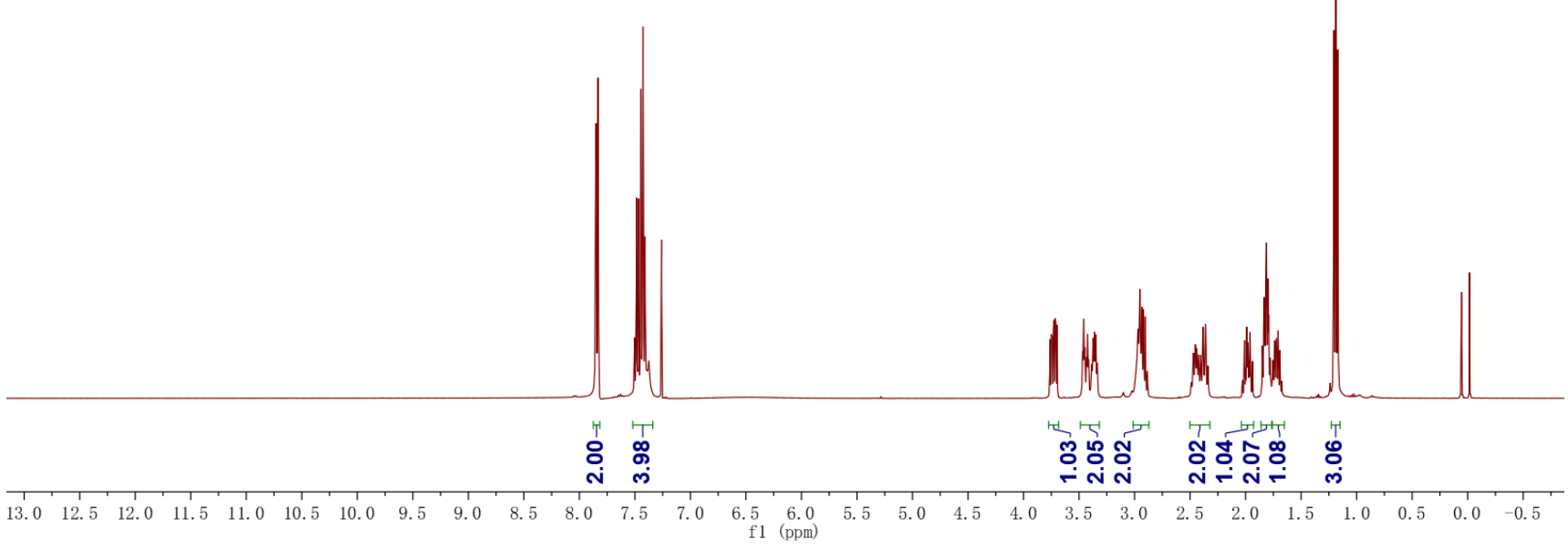

(1)

${ }^{13} \mathrm{C} \mathrm{NMR}\left(100 \mathrm{MHz}, \mathrm{CDCl}_{3}\right)$

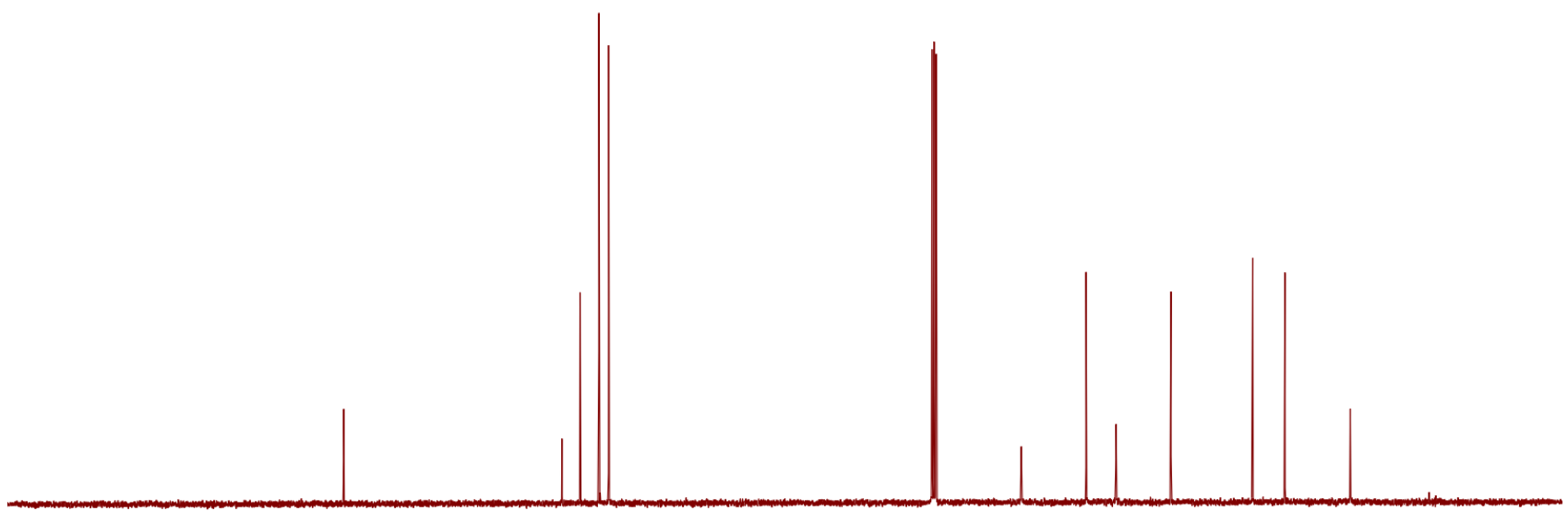

$\begin{array}{llllllllllll}210 & 200 & 190 & 180 & 170 & 160 & 150 & 140 & 130 & 120 & 110 & \begin{array}{l}100 \\ \mathrm{f} 1(\mathrm{ppm})\end{array}\end{array}$

Figure S40. ${ }^{1} \mathrm{H}$ NMR and ${ }^{13} \mathrm{C}\left\{{ }^{1} \mathrm{H}\right\}$ NMR Spectra of $N$-((1-ethylpyrrolidin-2-yl)methyl)benzamide (4r). 
${ }^{1} \mathrm{H}$ NMR (400 MHz, $\left.\mathrm{CDCl}_{3}\right)$
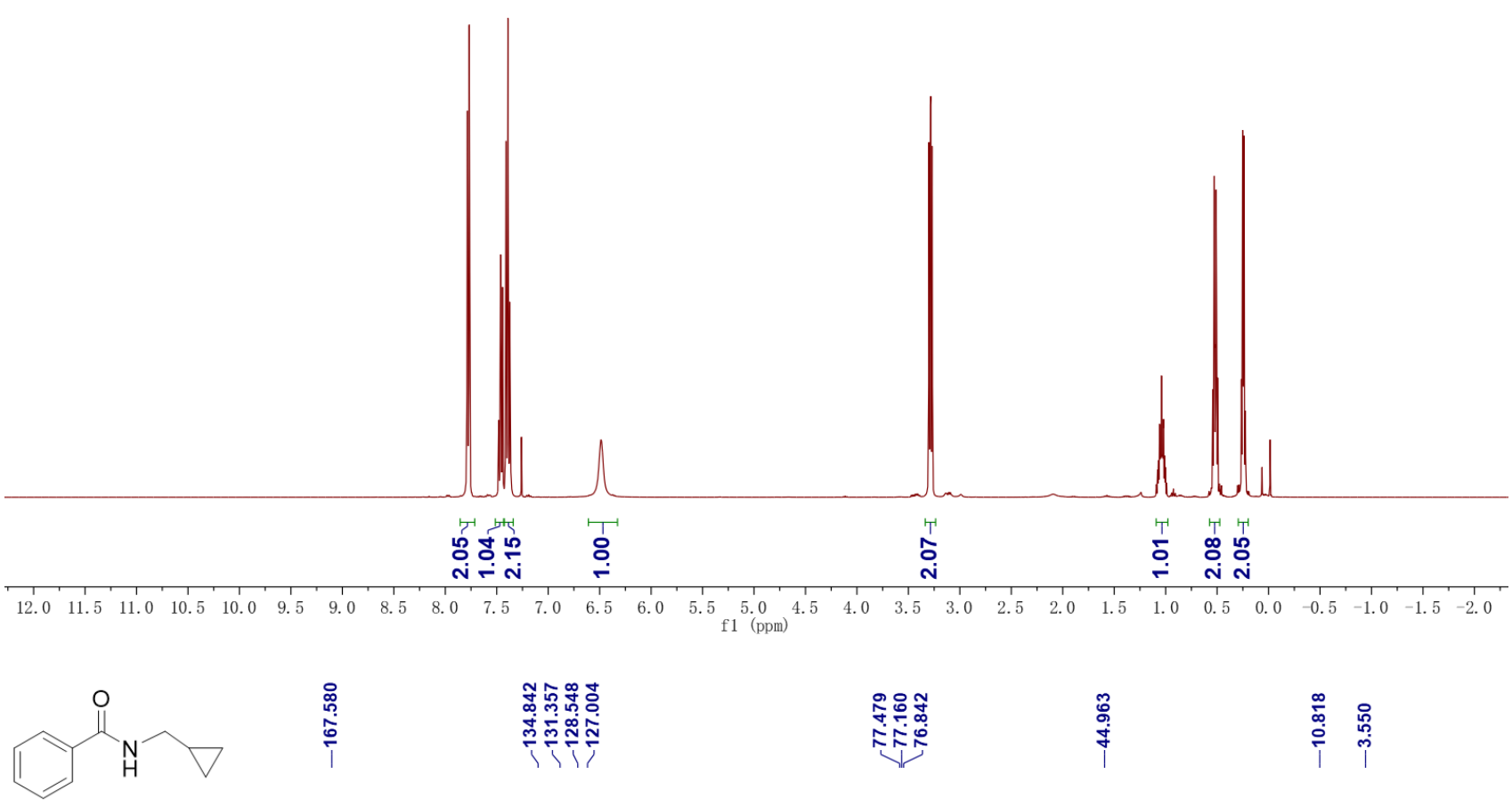

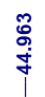

$\underset{\substack{\infty \\ \infty}}{\substack{\infty \\ 0}}$

${ }^{13} \mathrm{C} \mathrm{NMR}\left(100 \mathrm{MHz}, \mathrm{CDCl}_{3}\right)$

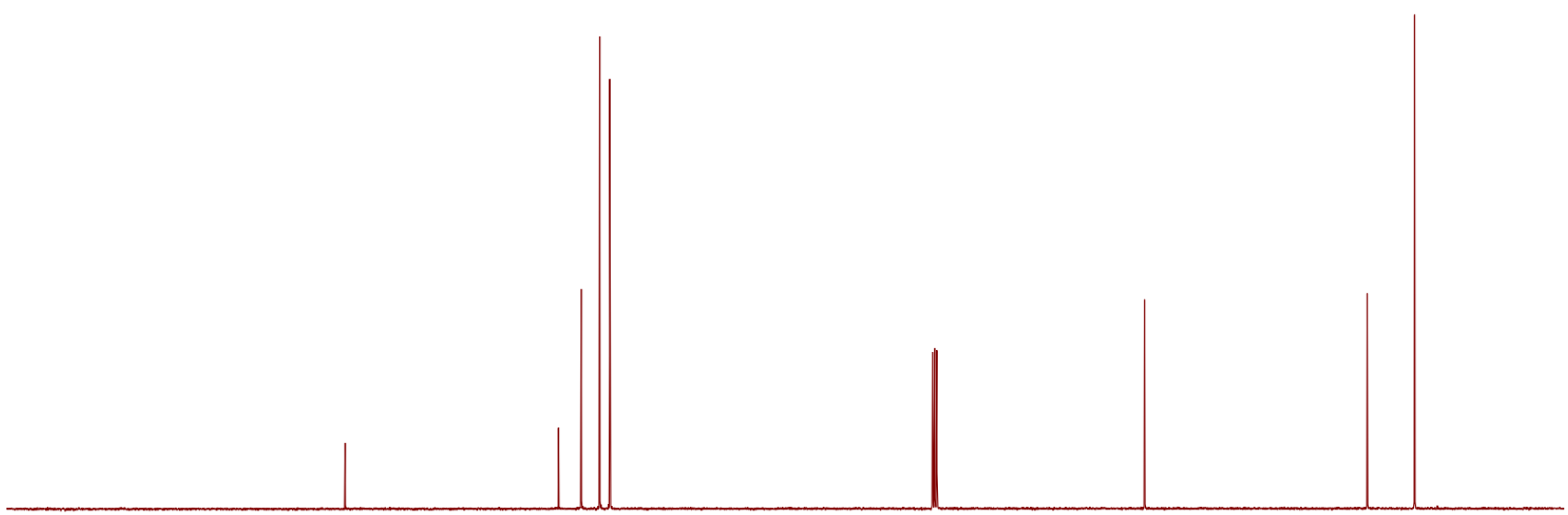

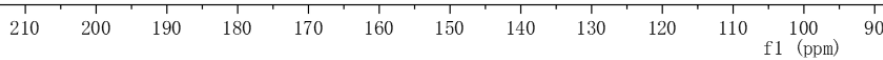

Figure S41. ${ }^{1} \mathrm{H}$ NMR and ${ }^{13} \mathrm{C}\left\{{ }^{1} \mathrm{H}\right\}$ NMR Spectra of $N$-(cyclopropylmethyl)benzamide (4s). 
${ }_{\mathrm{Hh}}^{\mathrm{O}} \stackrel{\mathrm{O}}{\mathrm{N}}$

${ }^{1} \mathrm{H}$ NMR (400 MHz, $\left.\mathrm{CDCl}_{3}\right)$

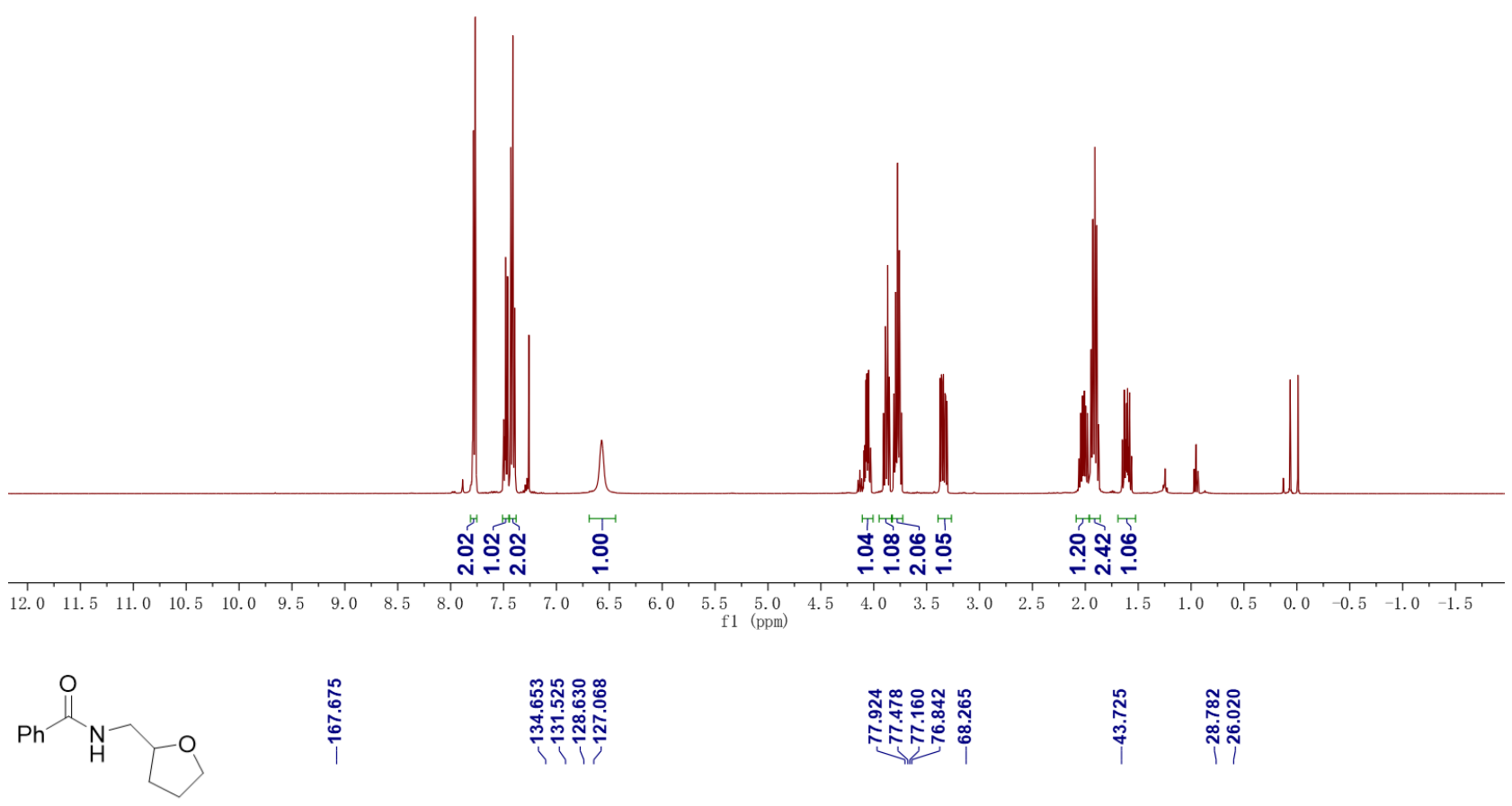

${ }^{13} \mathrm{C}$ NMR $\left(100 \mathrm{MHz}, \mathrm{CDCl}_{3}\right)$

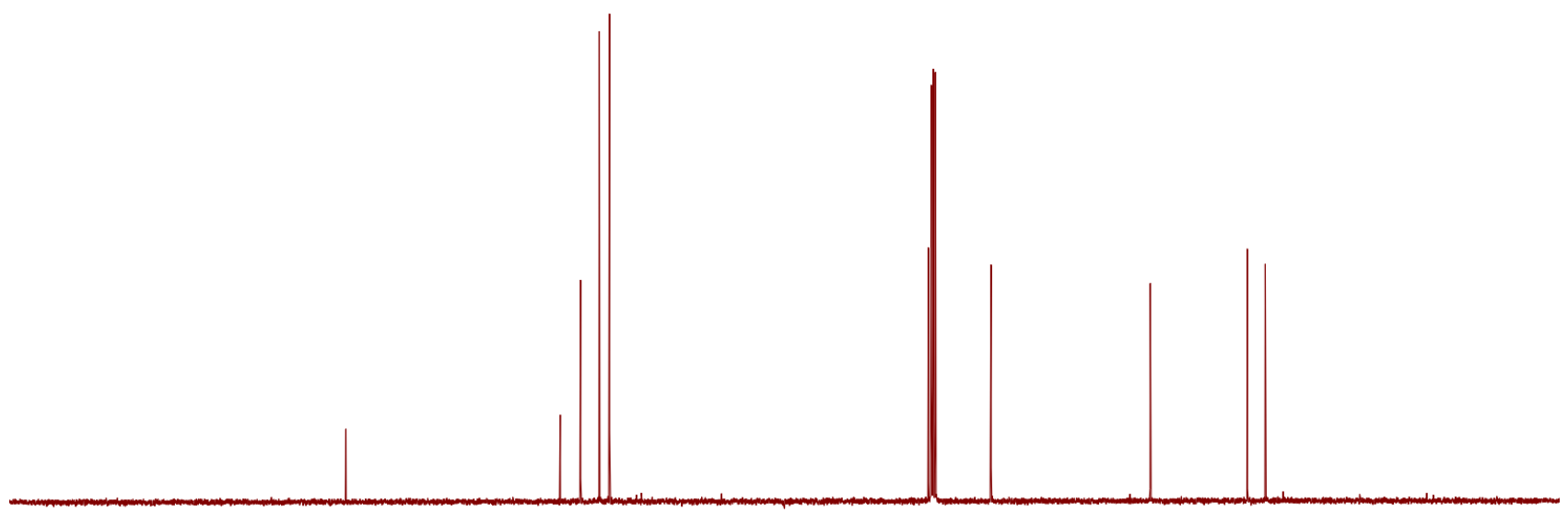

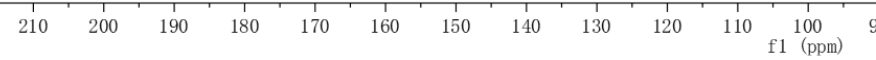

Figure S42. ${ }^{1} \mathrm{H}$ NMR and ${ }^{13} \mathrm{C}\left\{{ }^{1} \mathrm{H}\right\}$ NMR Spectra of $N$-((tetrahydrofuran-2-yl)methyl)benzamide (4t). 
${ }^{1} \mathrm{H}$ NMR (400 MHz, $\left.\mathrm{CDCl}_{3}\right)$

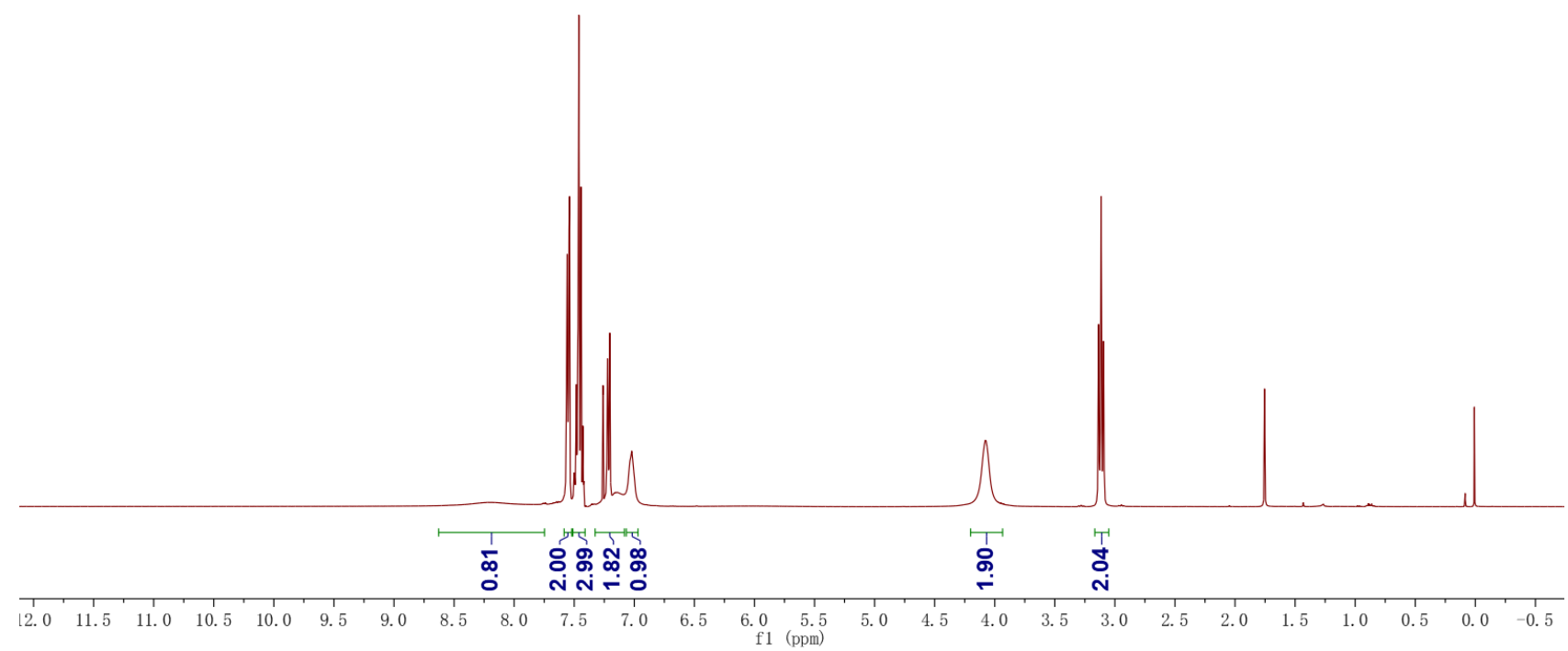

勧

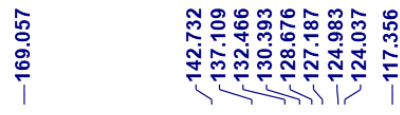

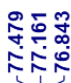

总

${ }^{13} \mathrm{C} \mathrm{NMR}\left(100 \mathrm{MHz}, \mathrm{CDCl}_{3}\right)$

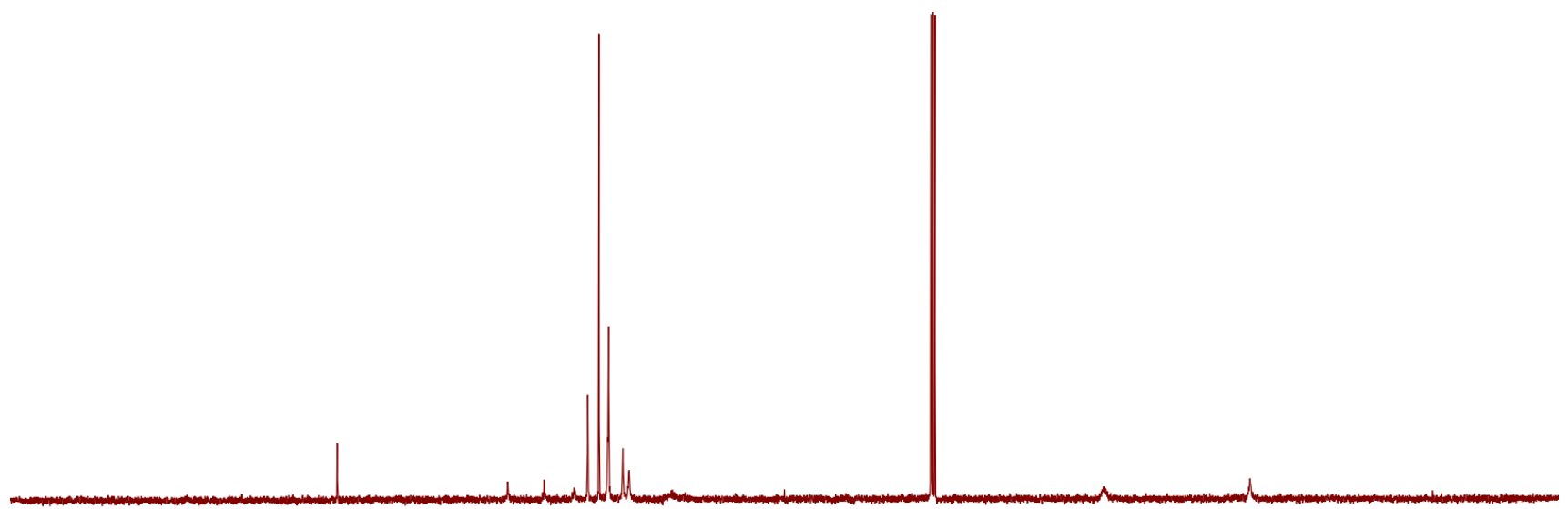

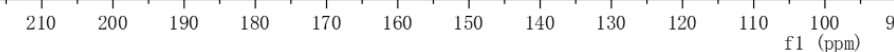

Figure S43. ${ }^{1} \mathrm{H}$ NMR and ${ }^{13} \mathrm{C}\left\{{ }^{1} \mathrm{H}\right\}$ NMR Spectra of indolin-1-yl(phenyl)methanone (4u). 


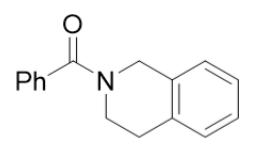

${ }^{1} \mathrm{H}$ NMR (400 MHz, $\left.\mathrm{C}_{6} \mathrm{D}_{6}\right)$

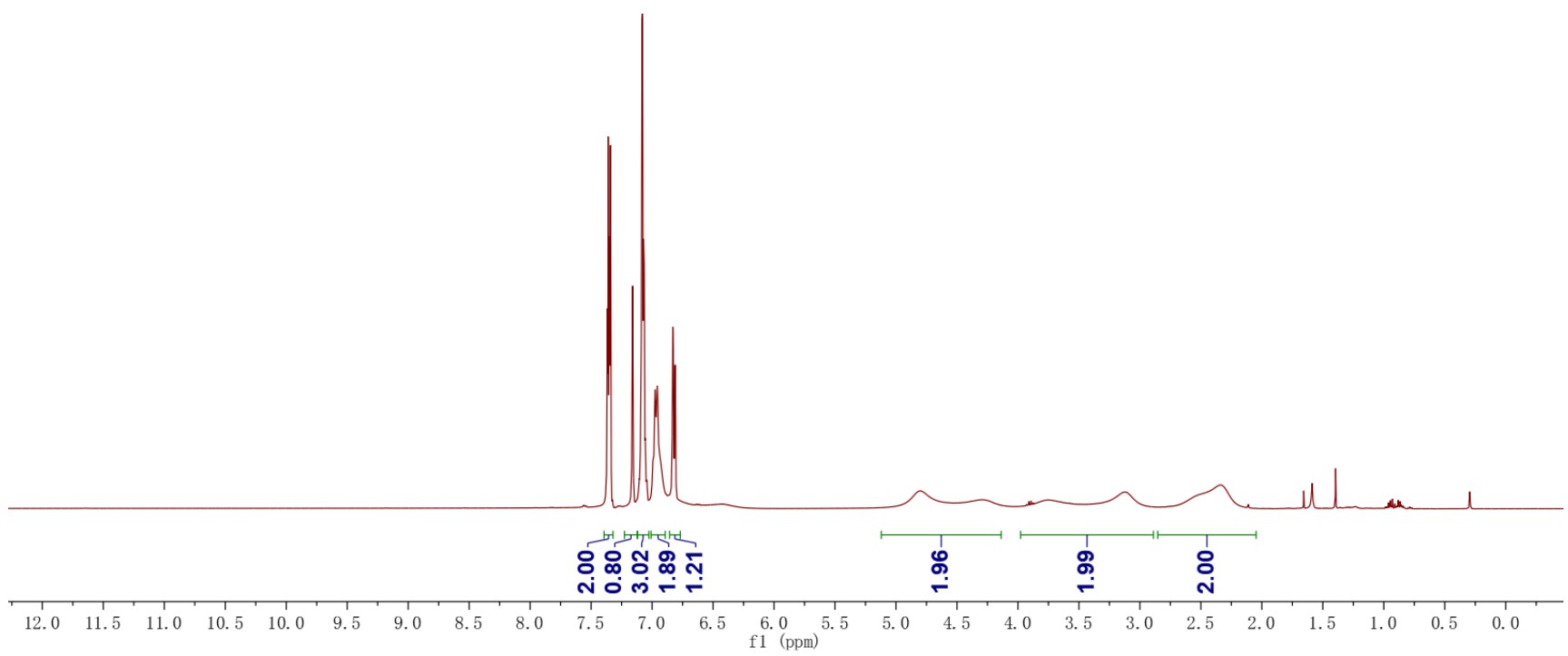<smiles>O=C(c1ccccc1)N1CCc2ccccc2C1</smiles>

${ }^{13} \mathrm{C}$ NMR (100 MHz, $\left.\mathrm{C}_{6} \mathrm{D}_{6}\right)$

Figure S44. ${ }^{1} \mathrm{H}$ NMR and ${ }^{13} \mathrm{C}\left\{{ }^{1} \mathrm{H}\right\}$ NMR Spectra of (3,4-dihydroisoquinolin-2(1H)-yl)(phenyl)methanone (4v). 
篮

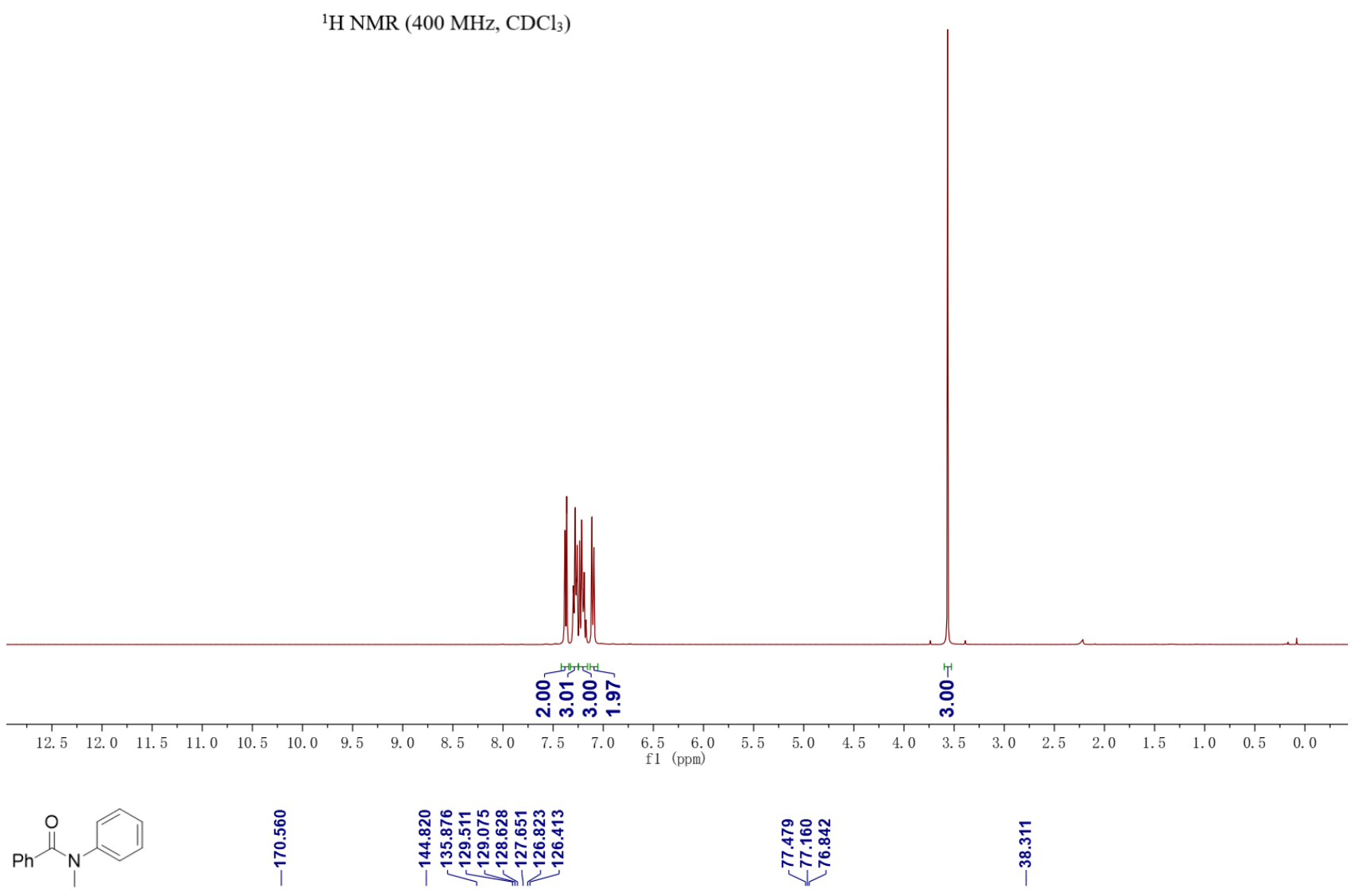

${ }^{13} \mathrm{C}$ NMR $\left(100 \mathrm{MHz}, \mathrm{CDCl}_{3}\right)$

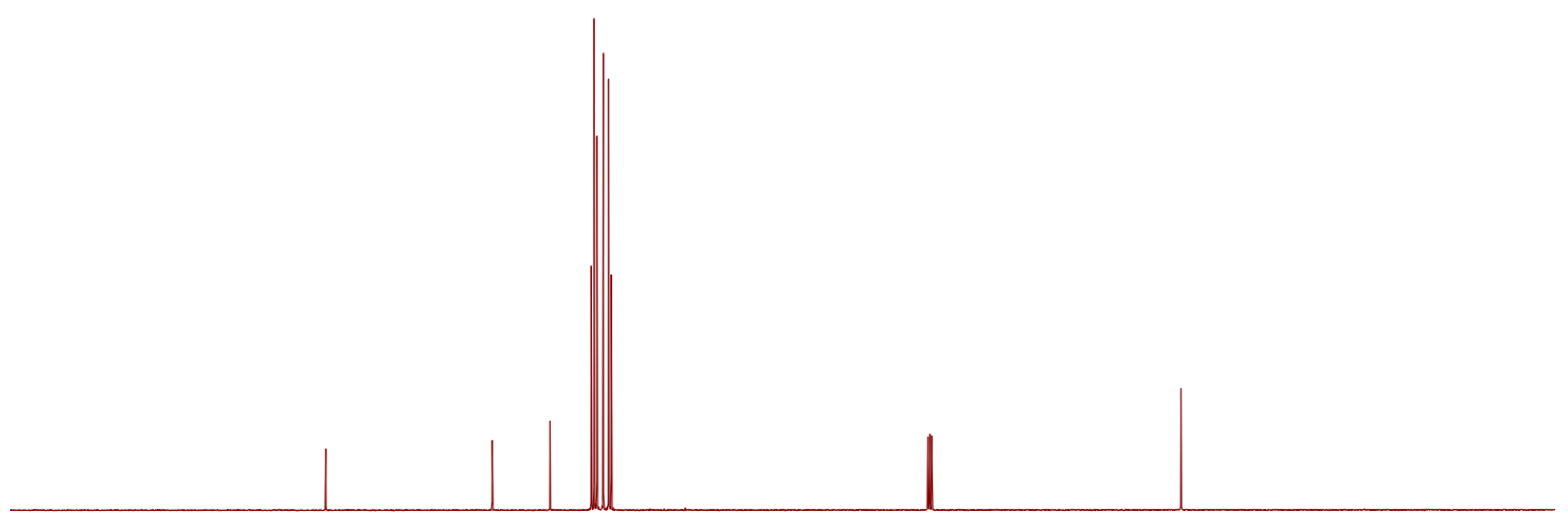

Figure S45. ${ }^{1} \mathrm{H}$ NMR and ${ }^{13} \mathrm{C}\left\{{ }^{1} \mathrm{H}\right\}$ NMR Spectra of $N$-methyl- $N$-phenylbenzamide (4w). 


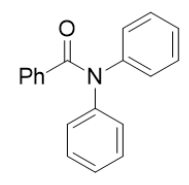

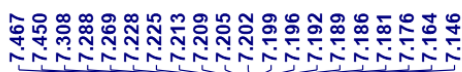

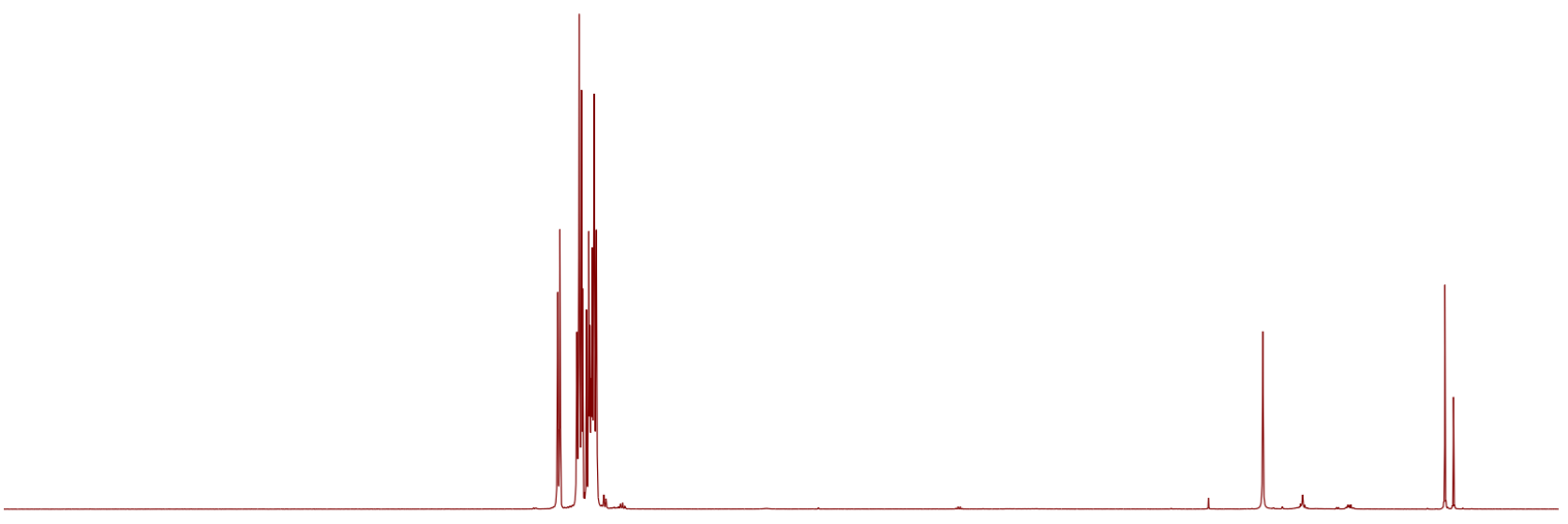

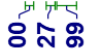

ก่

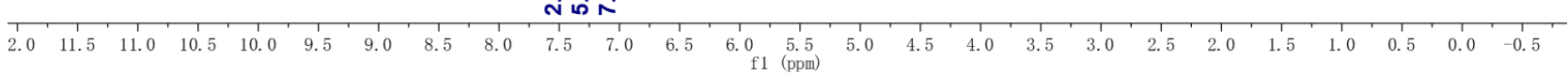

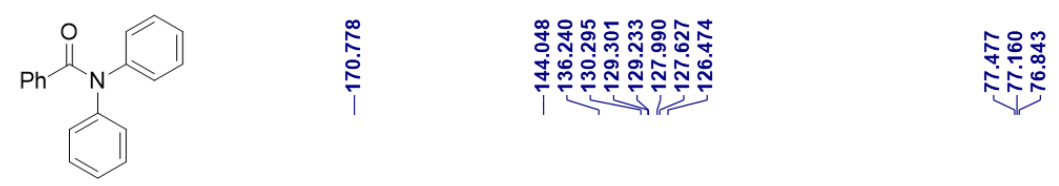

${ }^{13} \mathrm{C}$ NMR $\left(100 \mathrm{MHz}, \mathrm{CDCl}_{3}\right)$

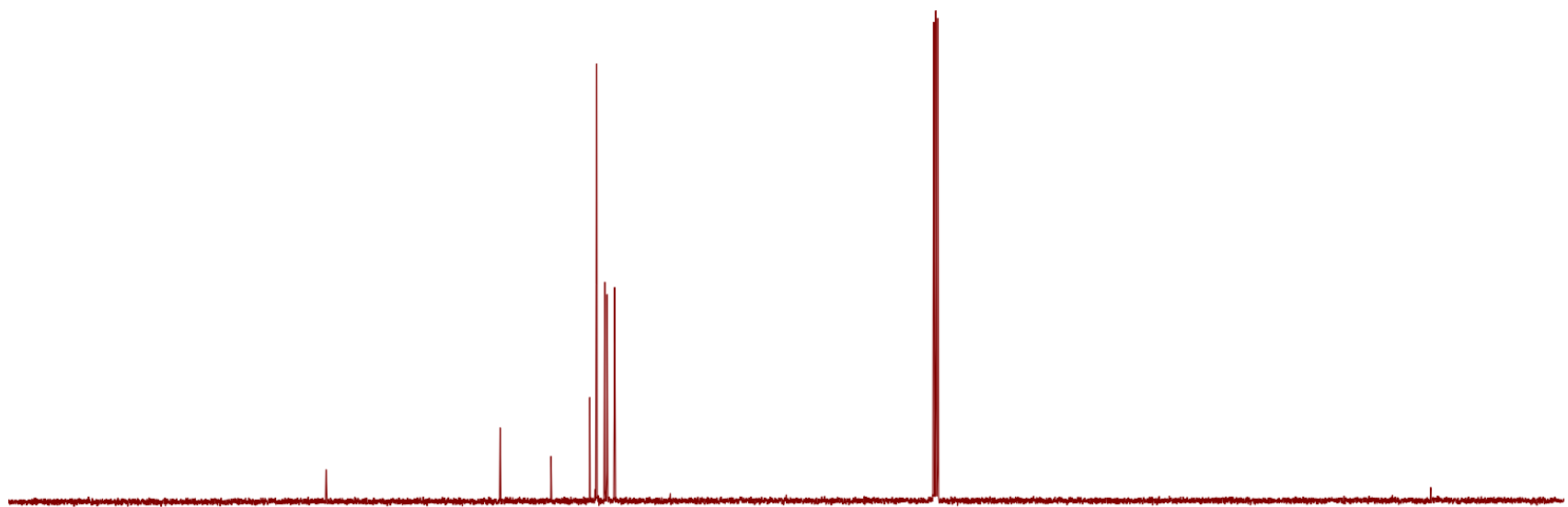

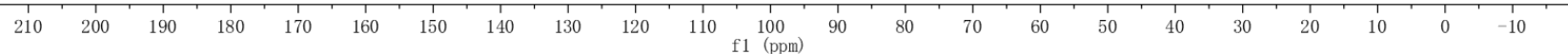

Figure S46. ${ }^{1} \mathrm{H}$ NMR and ${ }^{13} \mathrm{C}\left\{{ }^{1} \mathrm{H}\right\}$ NMR Spectra of $N$, $N$-diphenylbenzamide $(\mathbf{4 x})$. 


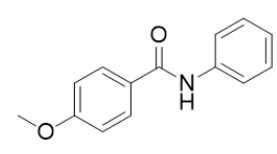

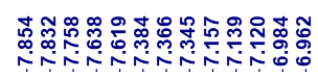

${ }^{1} \mathrm{H}$ NMR $\left(400 \mathrm{MHz}, \mathrm{CDCl}_{3}\right)$

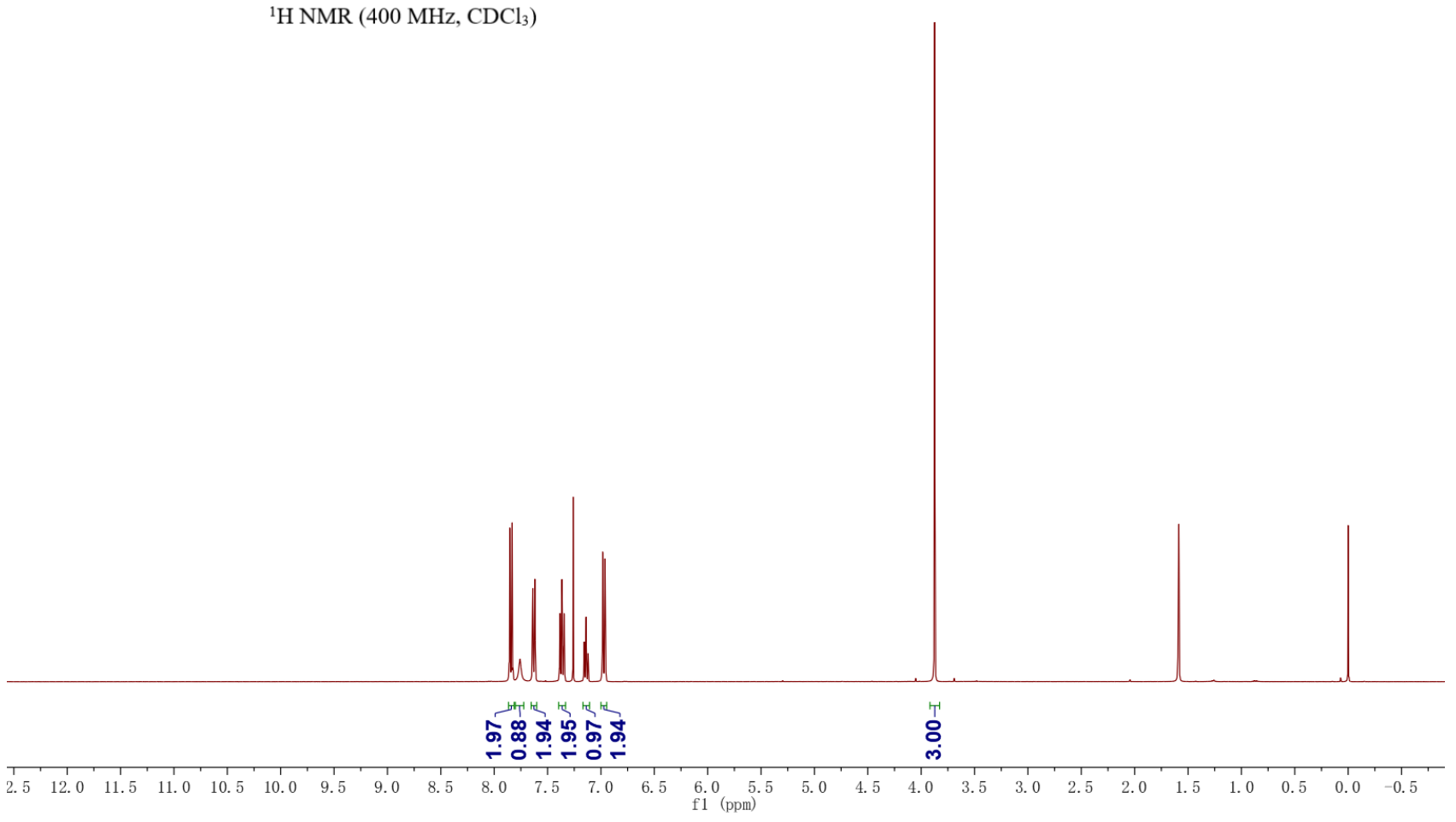

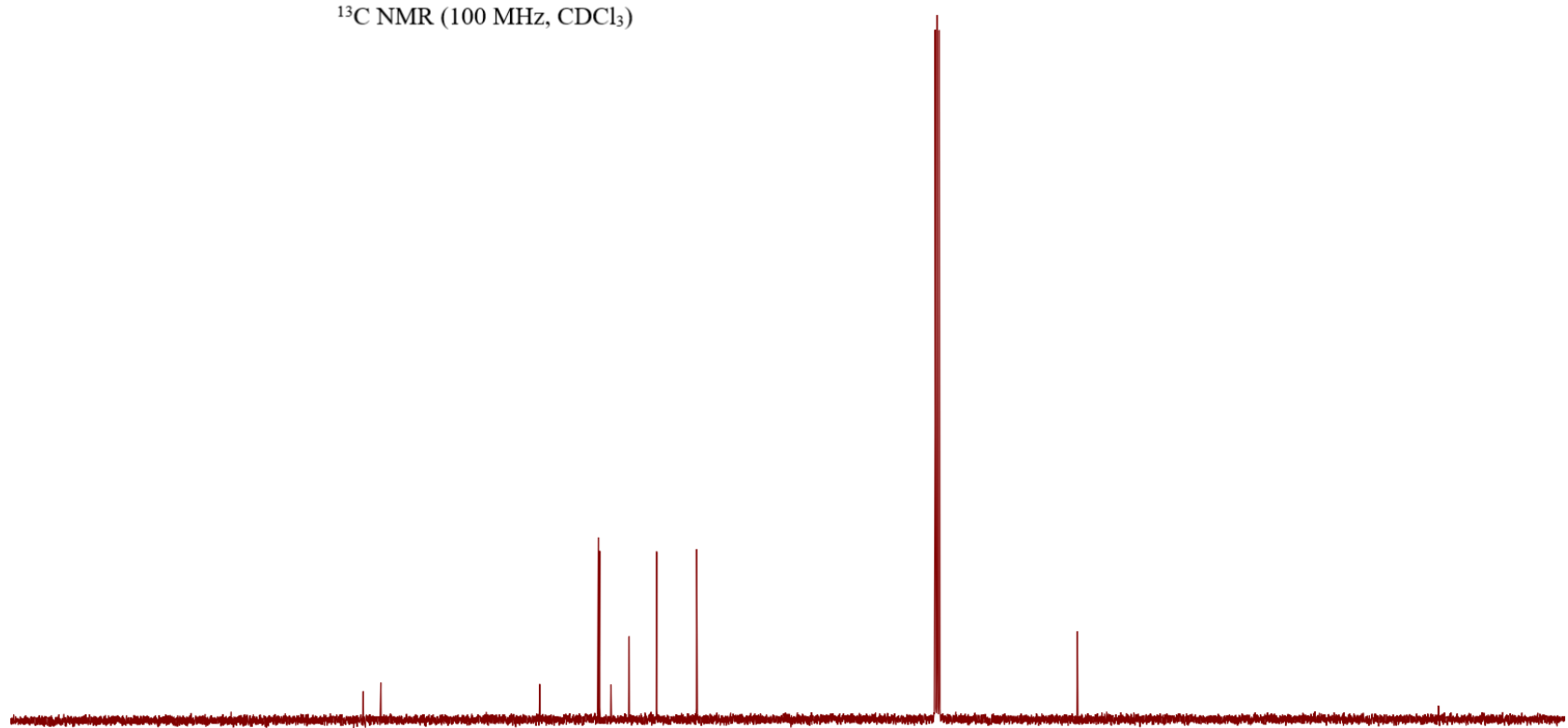

$\begin{array}{llllllllllll}210 & 200 & 190 & 180 & 170 & 160 & 150 & 140 & 130 & 120 & 110 & 100 \\ \mathrm{f} 1 & (\mathrm{pp})\end{array}$

Figure S47. ${ }^{1} \mathrm{H}$ NMR and ${ }^{13} \mathrm{C}\left\{{ }^{1} \mathrm{H}\right\}$ NMR Spectra of 4-methoxy- $N$-phenylbenzamide (5a). 

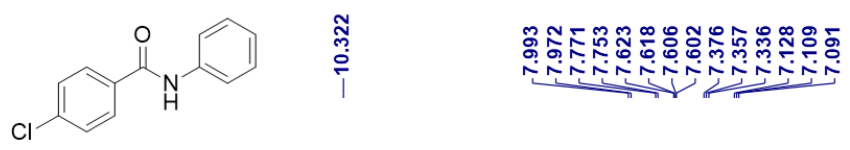

${ }^{1} \mathrm{H}$ NMR (400 MHz, DMSO-d $\left.d_{6}\right)$
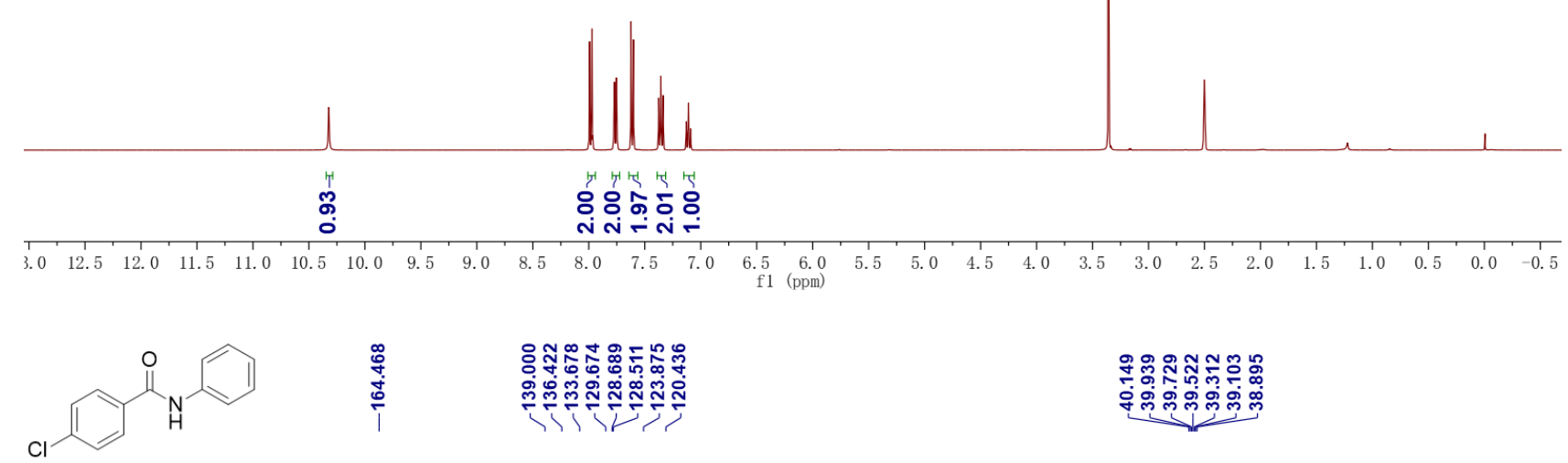

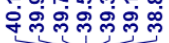

${ }^{13} \mathrm{C}$ NMR (400 MHz, DMSO- $d_{6}$ )

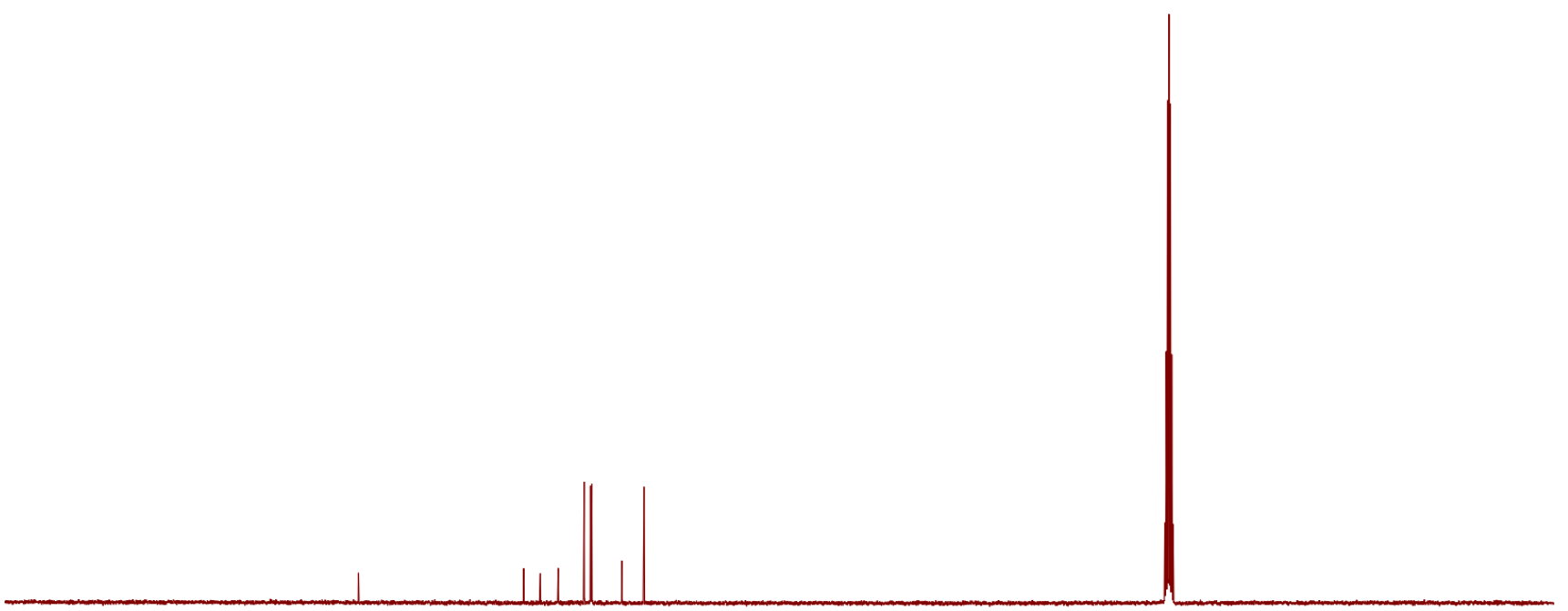

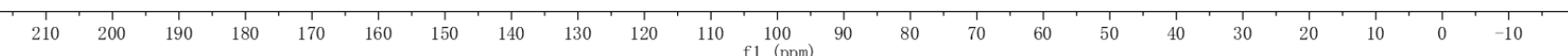

Figure S48. ${ }^{1} \mathrm{H}$ NMR and ${ }^{13} \mathrm{C}\left\{{ }^{1} \mathrm{H}\right\}$ NMR Spectra of 4-chloro-N-phenylbenzamide (5b). 
<smiles>CC(NC(=O)c1ccccc1)C1CCCC1</smiles>

${ }^{1} \mathrm{H}$ NMR $\left(400 \mathrm{MHz}, \mathrm{CDCl}_{3}\right)$

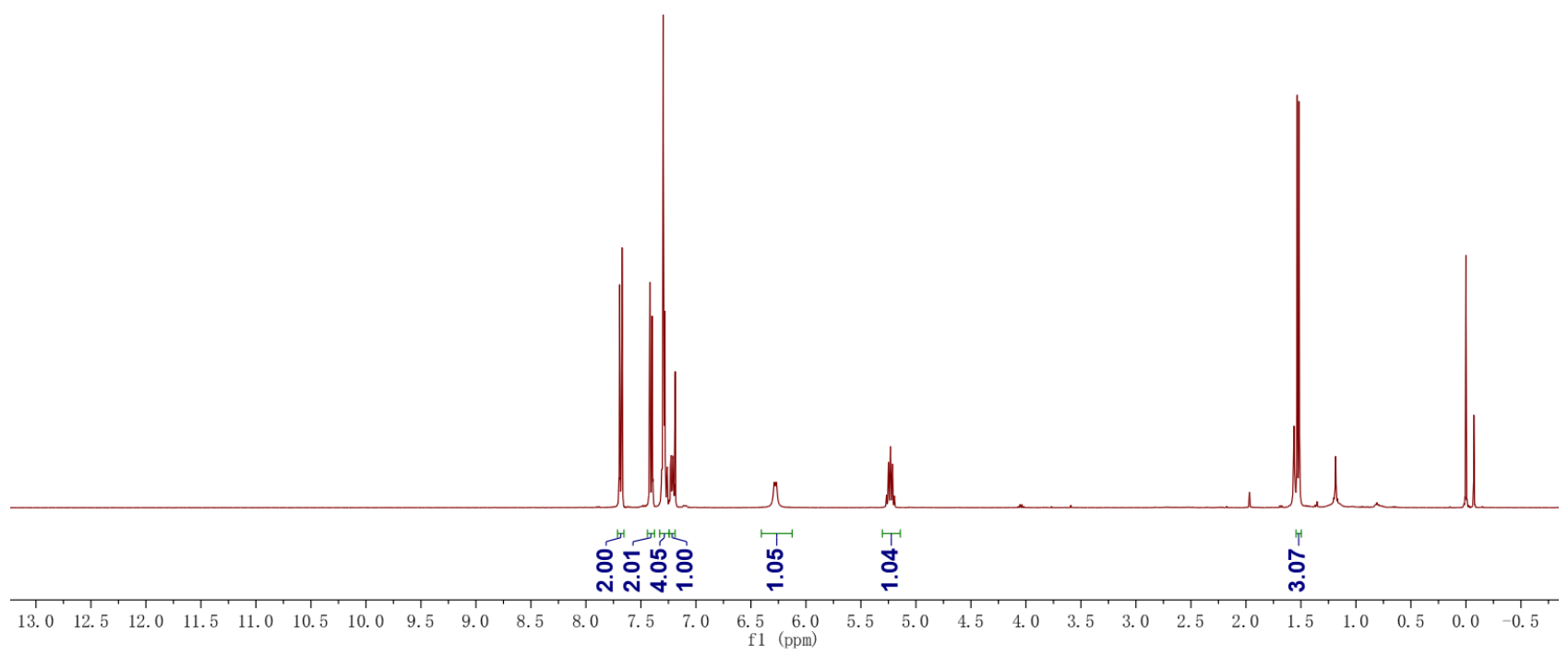

|ll|l

${ }^{13} \mathrm{C} \mathrm{NMR}\left(100 \mathrm{MHz}, \mathrm{CDCl}_{3}\right)$

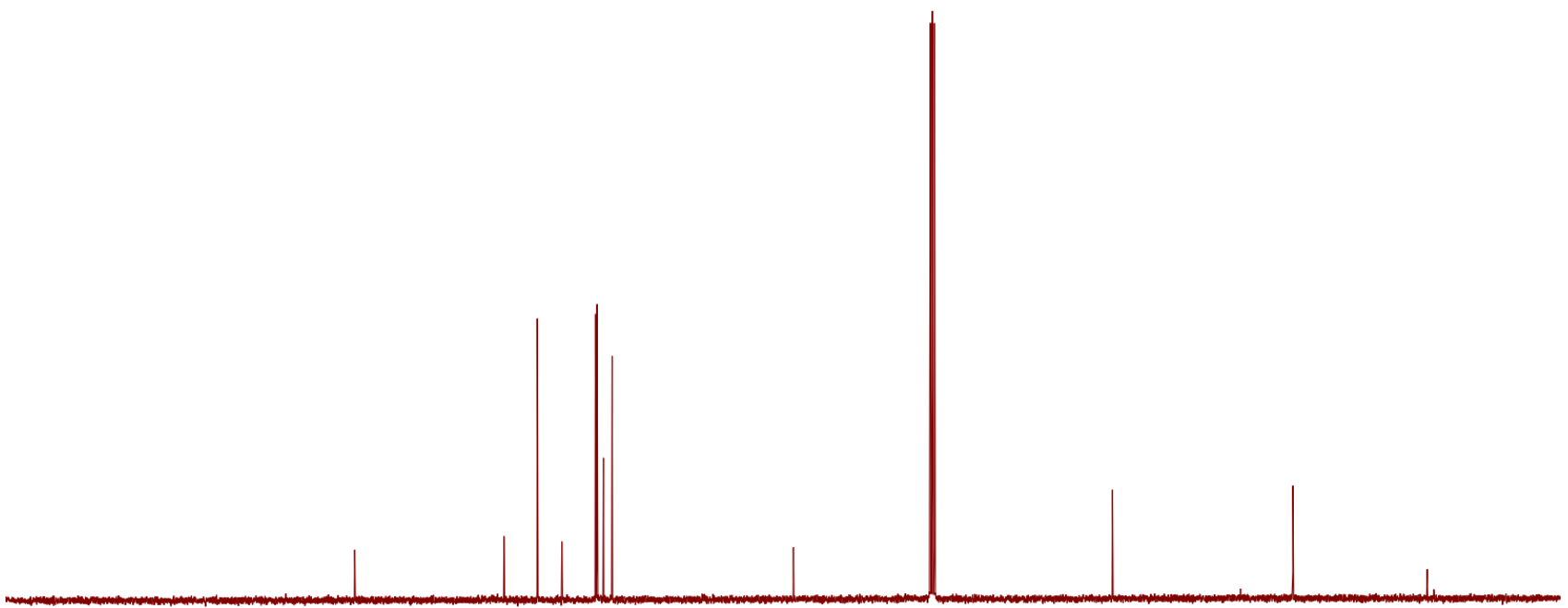

$\begin{array}{llllllllllll}210 & 200 & 190 & 180 & 170 & 160 & 150 & 140 & 130 & 120 & 110 & \begin{array}{l}100 \\ \mathrm{f} 1\end{array}(\mathrm{ppm})\end{array}$

Figure S49. ${ }^{1} \mathrm{H}$ NMR and ${ }^{13} \mathrm{C}\left\{{ }^{1} \mathrm{H}\right\}$ NMR Spectra of 4-iodo- $N$-(1-phenylethyl)benzamide (5c). 


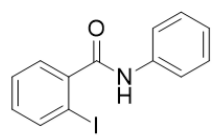

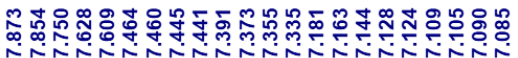

${ }^{1} \mathrm{H}$ NMR $\left(400 \mathrm{MHz}, \mathrm{CDCl}_{3}\right)$

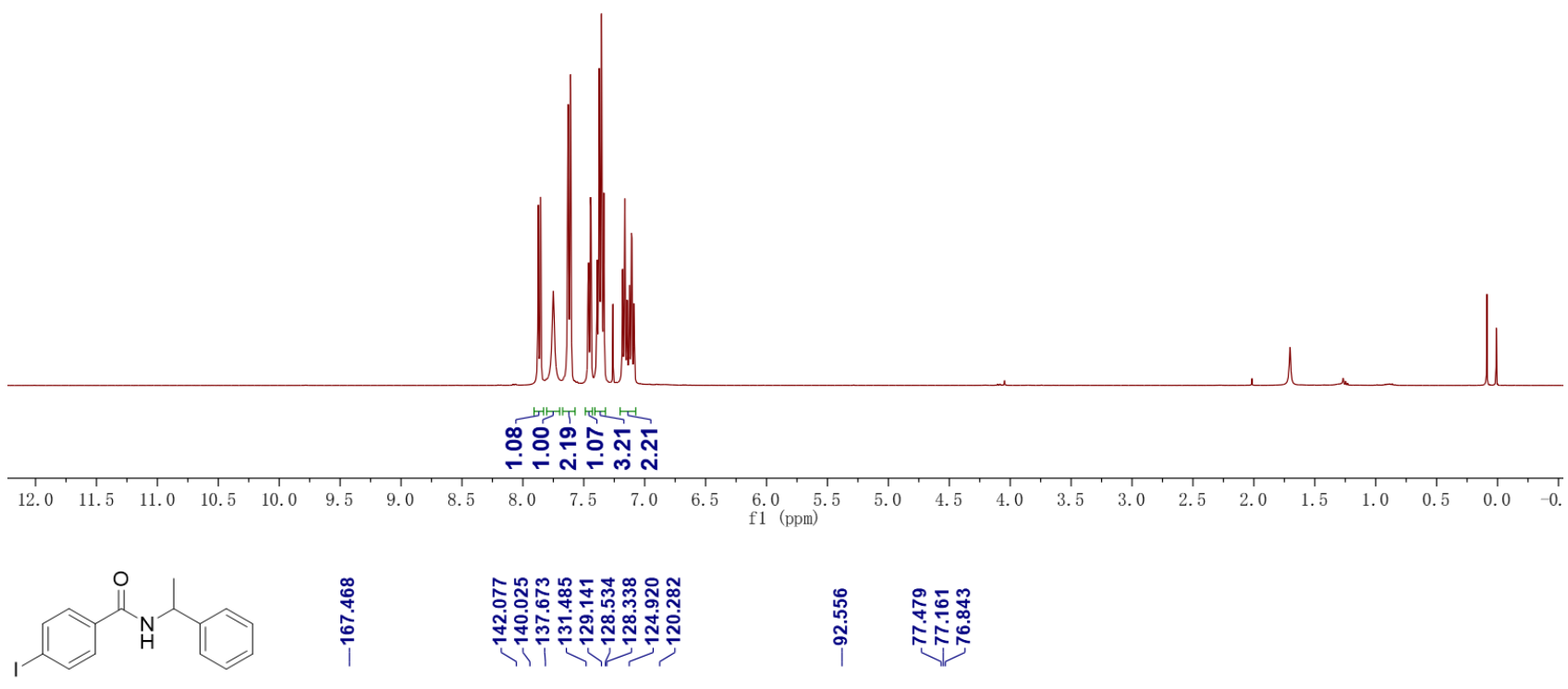

${ }^{13} \mathrm{C} \mathrm{NMR}\left(100 \mathrm{MHz}, \mathrm{CDCl}_{3}\right)$

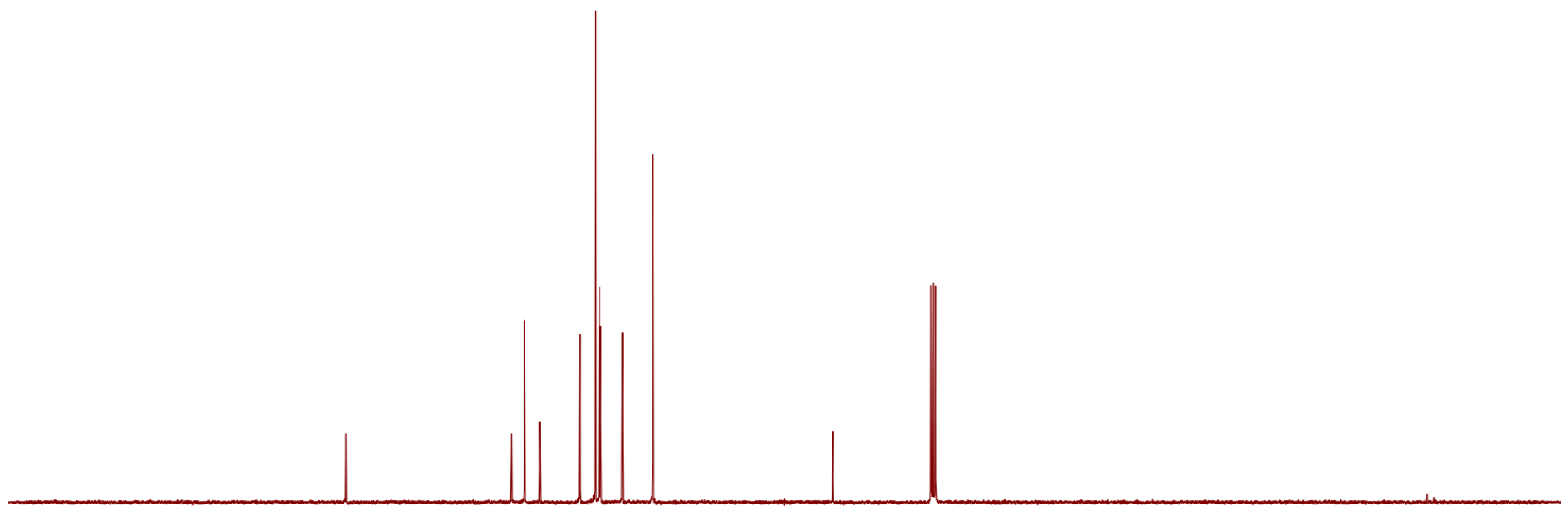

$\begin{array}{llllllllllll}210 & 200 & 190 & 180 & 170 & 160 & 150 & 140 & 130 & 120 & 110 & 100 \\ \mathrm{f} 1(\mathrm{pp})\end{array}$

Figure S50. ${ }^{1} \mathrm{H}$ NMR and ${ }^{13} \mathrm{C}\left\{{ }^{1} \mathrm{H}\right\}$ NMR Spectra of 2-iodo- $N$-phenylbenzamide (5d). 


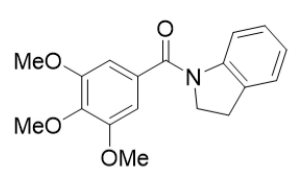

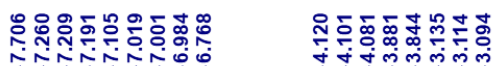

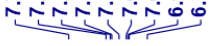

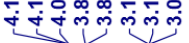

OMe

${ }^{1} \mathrm{H}$ NMR (400 MHz, $\mathrm{CDCl}_{3}$ )

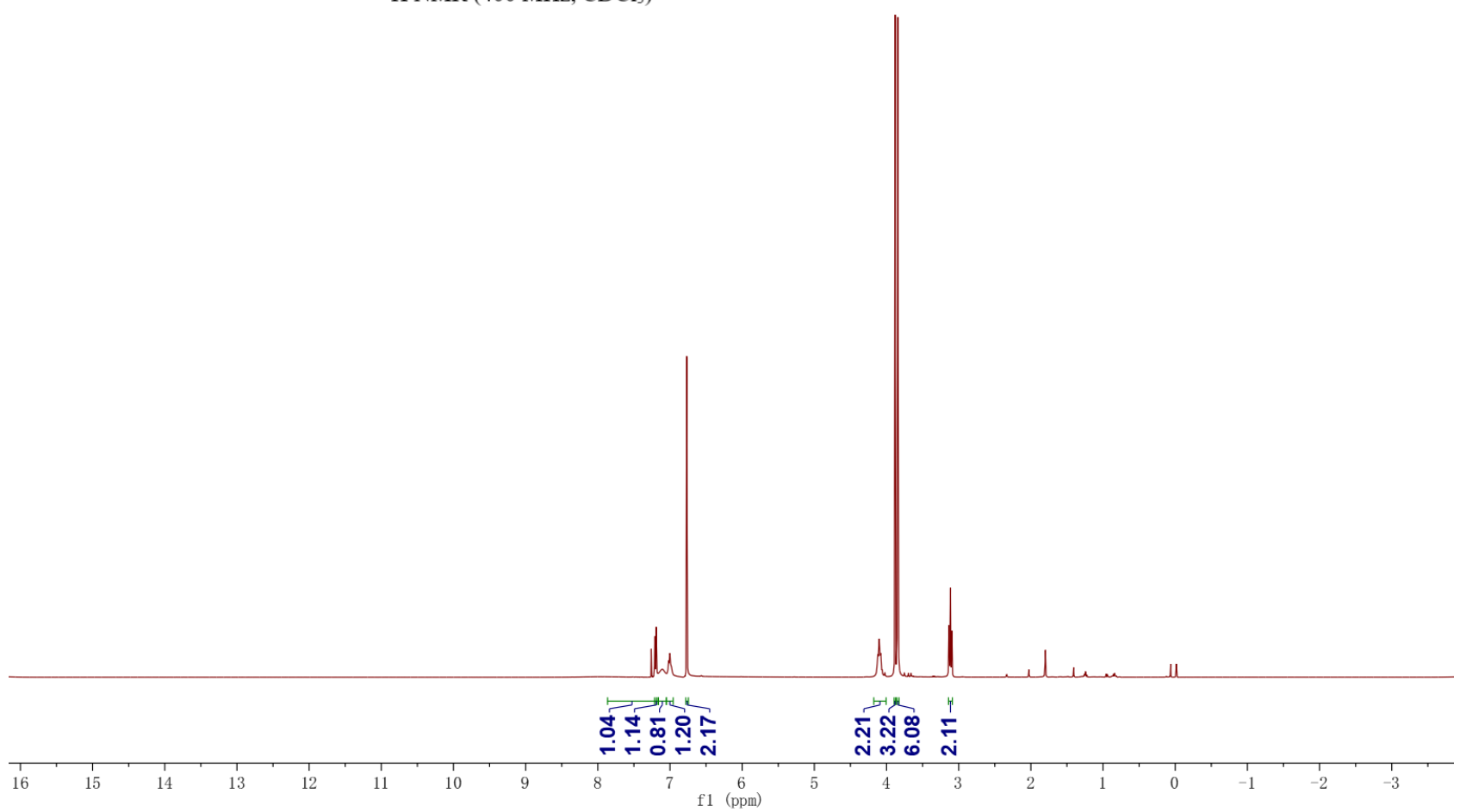

${ }^{13} \mathrm{C} \mathrm{NMR}\left(100 \mathrm{MHz}, \mathrm{CDCl}_{3}\right)$

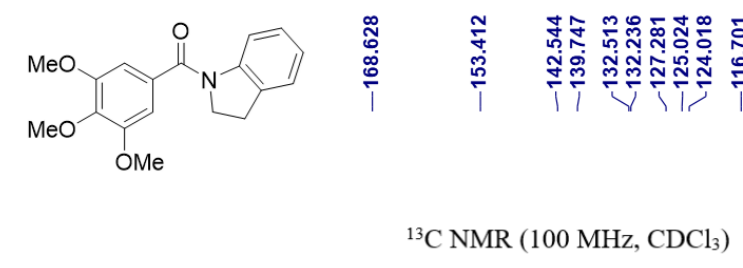

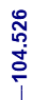

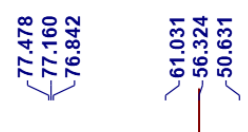

$\stackrel{\infty}{\infty}$

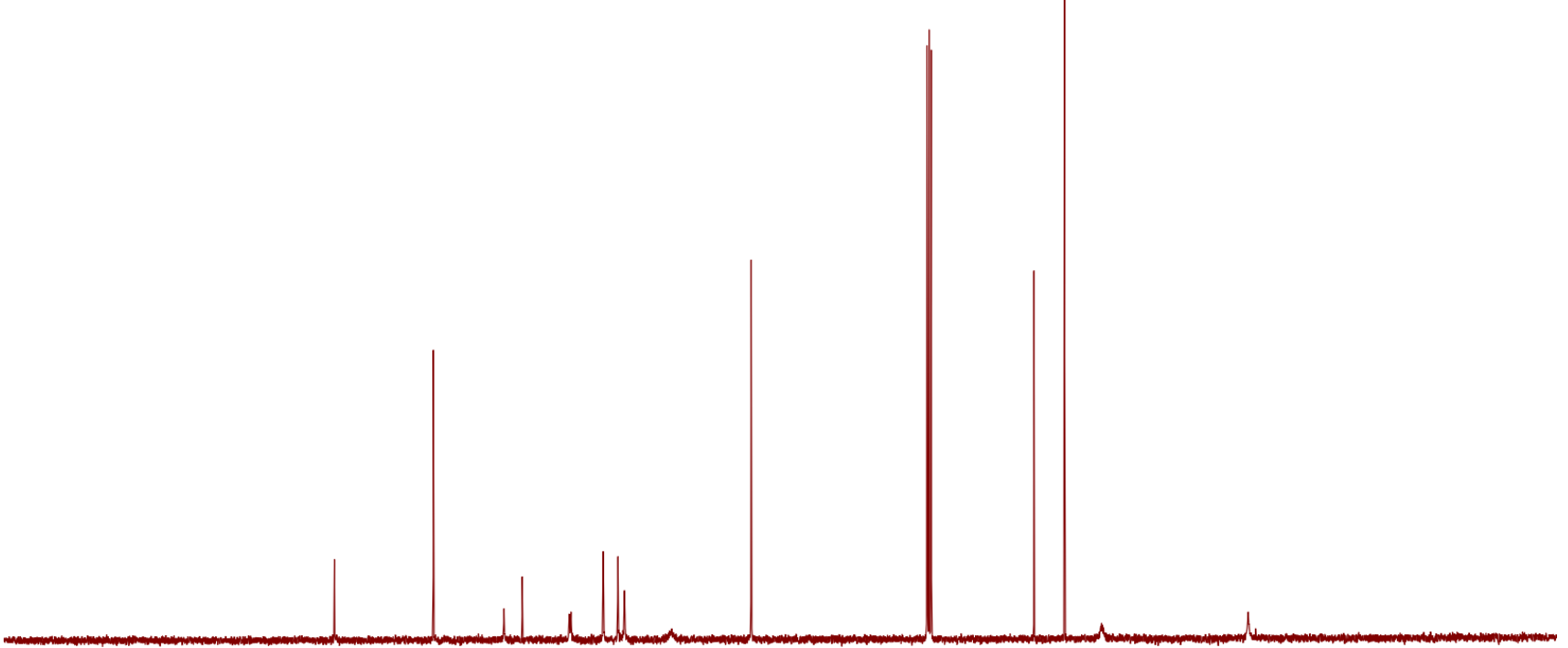

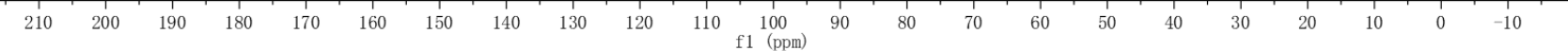

Figure S51. ${ }^{1} \mathrm{H}$ NMR and ${ }^{13} \mathrm{C}\left\{{ }^{1} \mathrm{H}\right\}$ NMR Spectra of indolin-1-yl(3,4,5-trimethoxyphenyl)methanone (5e). 


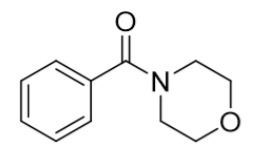

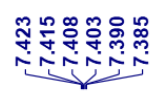

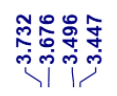

${ }^{1} \mathrm{H}$ NMR (400 MHz, $\left.\mathrm{CDCl}_{3}\right)$

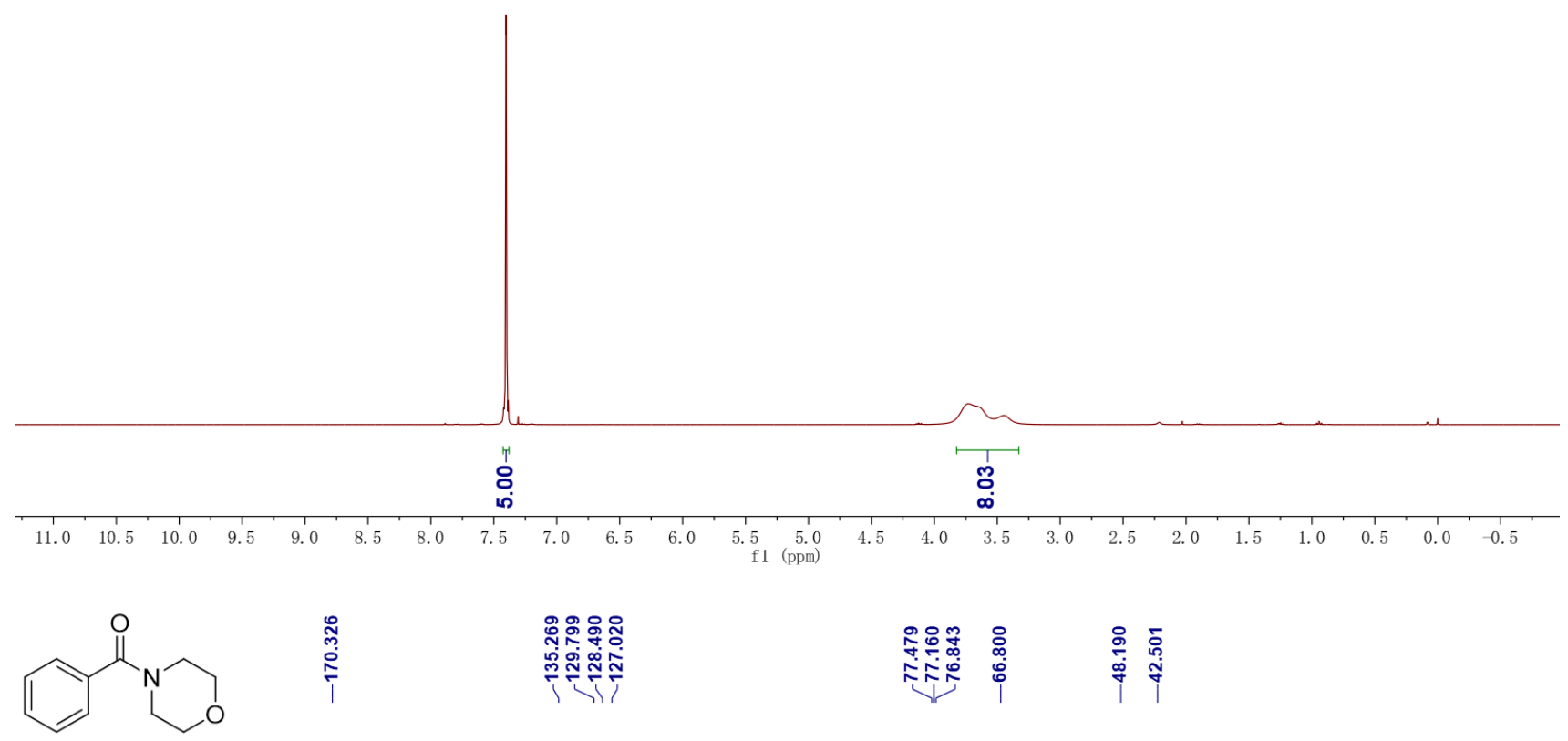

${ }^{13} \mathrm{C}$ NMR (100 MHz, $\left.\mathrm{CDCl}_{3}\right)$

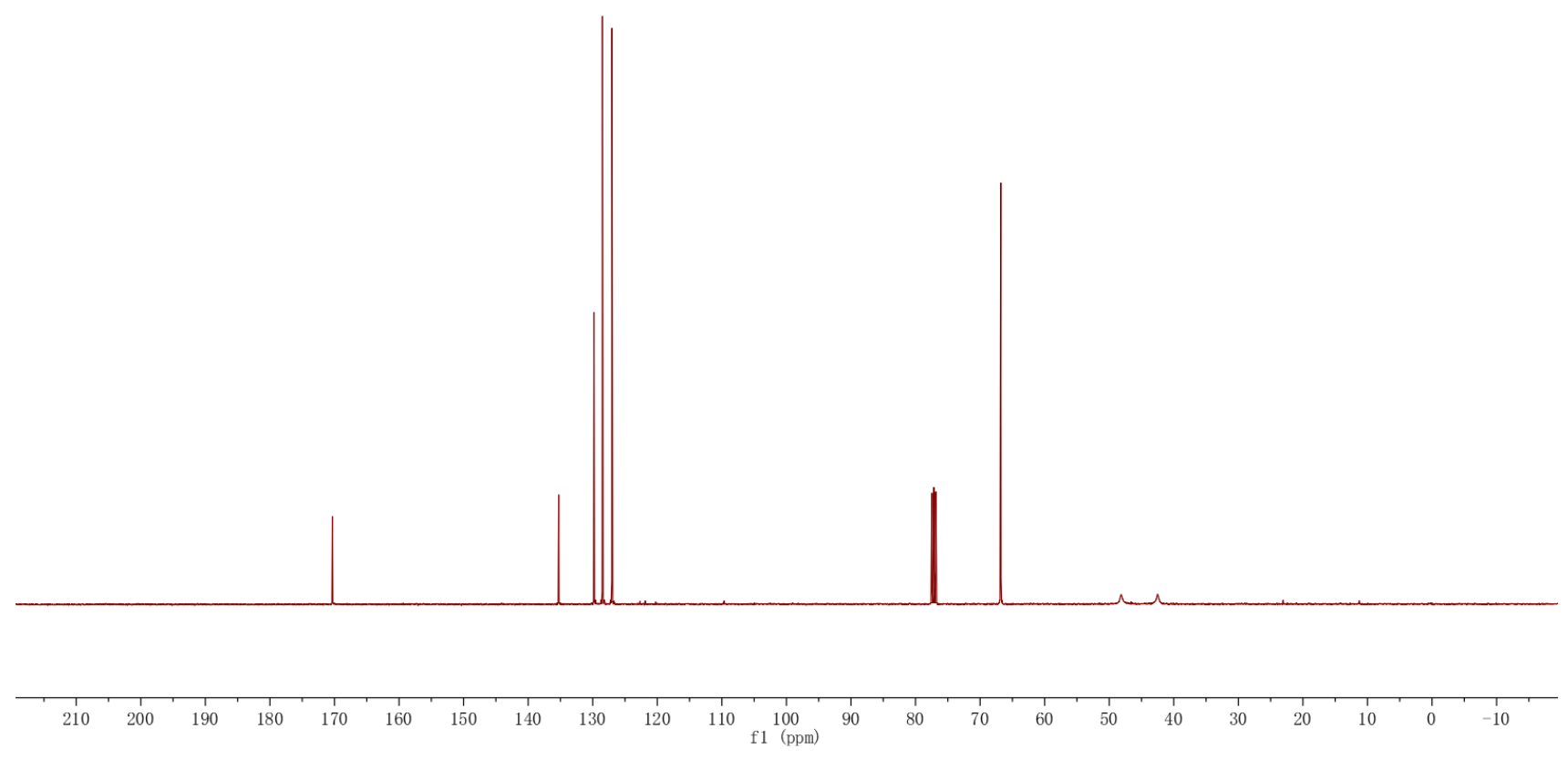

Figure S52. ${ }^{1} \mathrm{H}$ NMR and ${ }^{13} \mathrm{C}\left\{{ }^{1} \mathrm{H}\right\}$ NMR Spectra of 4-benzoylmorpholine (Ff).

S60 


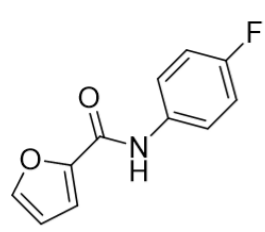

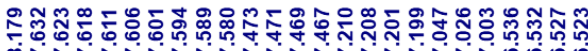

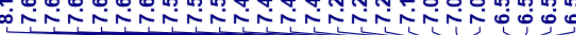

${ }^{1} \mathrm{H}$ NMR (400 MHz, $\left.\mathrm{CDCl}_{3}\right)$

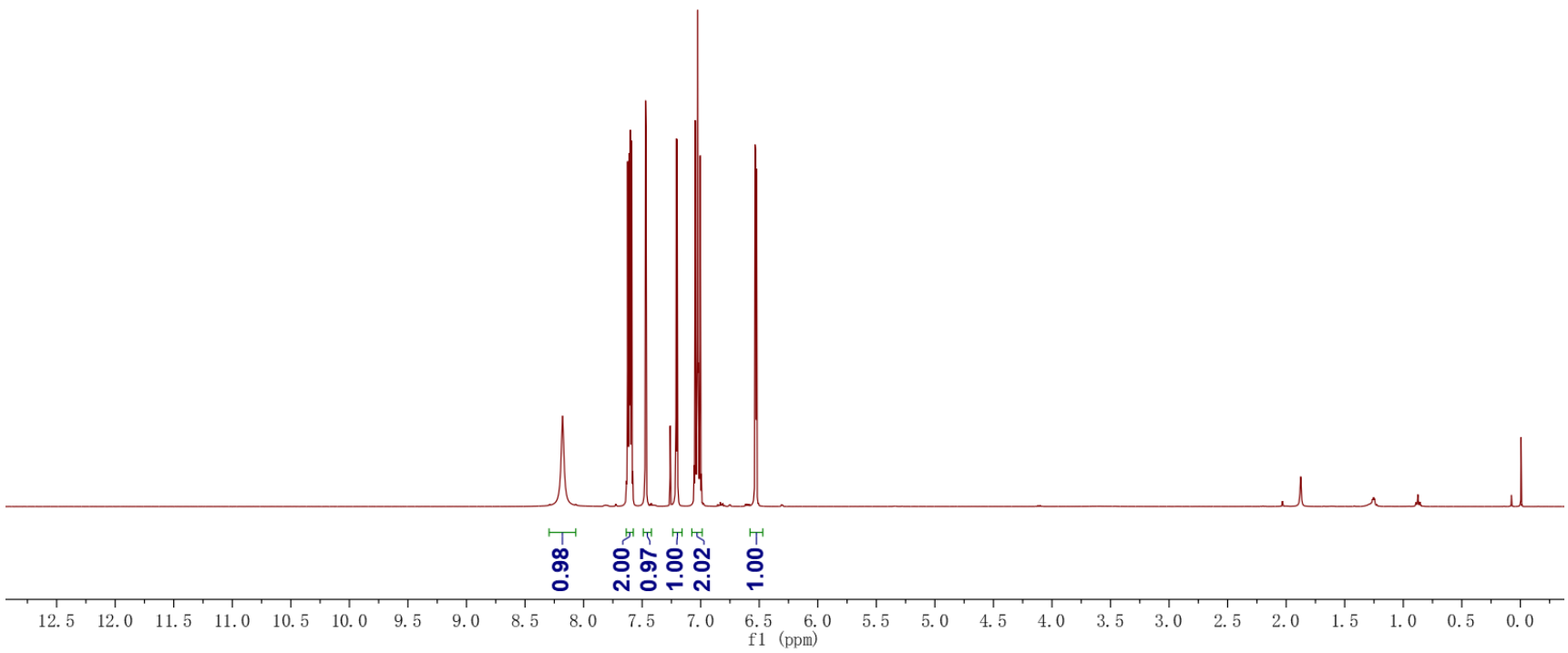<smiles>O=C(Nc1ccc(F)cc1)c1ccco1</smiles>

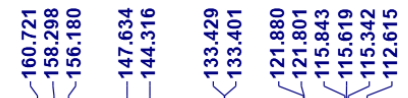

过范:

篎宾

${ }^{13} \mathrm{C} \mathrm{NMR}\left(100 \mathrm{MHz}, \mathrm{CDCl}_{3}\right)$

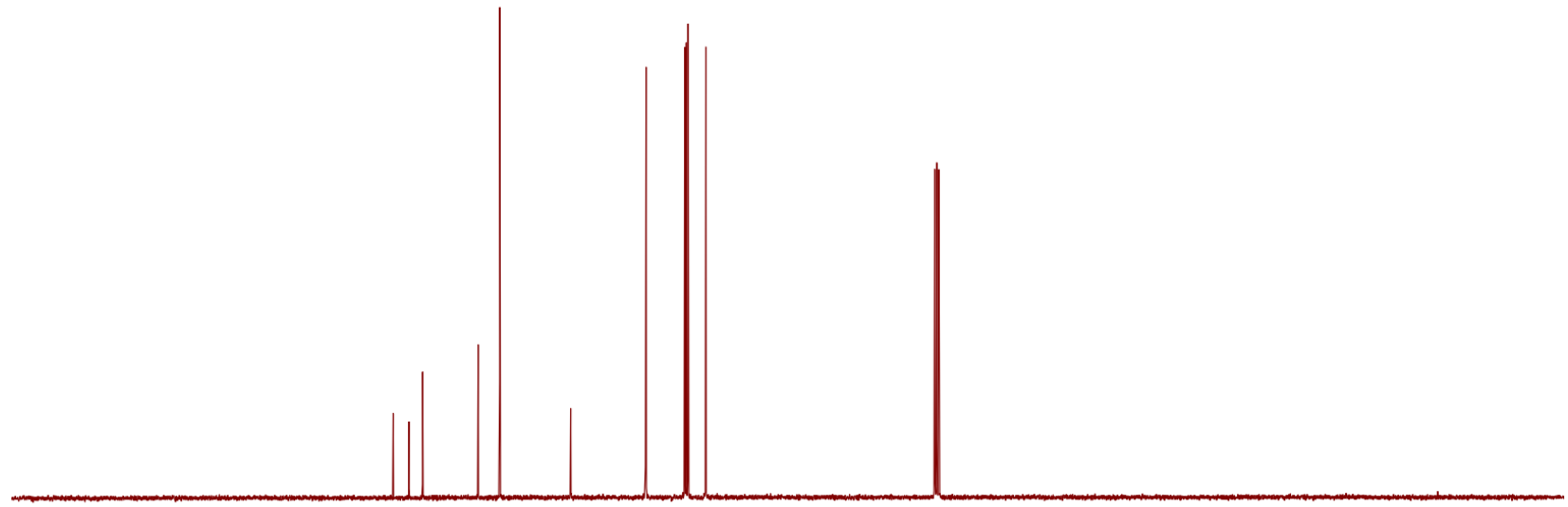

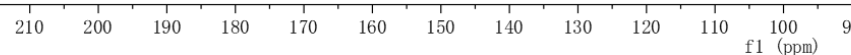

Figure S53. ${ }^{1} \mathrm{H}$ NMR and ${ }^{13} \mathrm{C}\left\{{ }^{1} \mathrm{H}\right\}$ NMR Spectra of $N$-(4-fluorophenyl)furan-2-carboxamide (5g). 


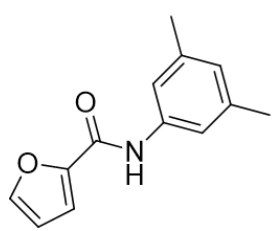

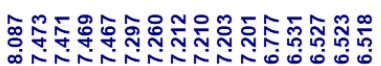

${ }^{1} \mathrm{H} \mathrm{NMR}\left(400 \mathrm{MHz}, \mathrm{CDCl}_{3}\right)$

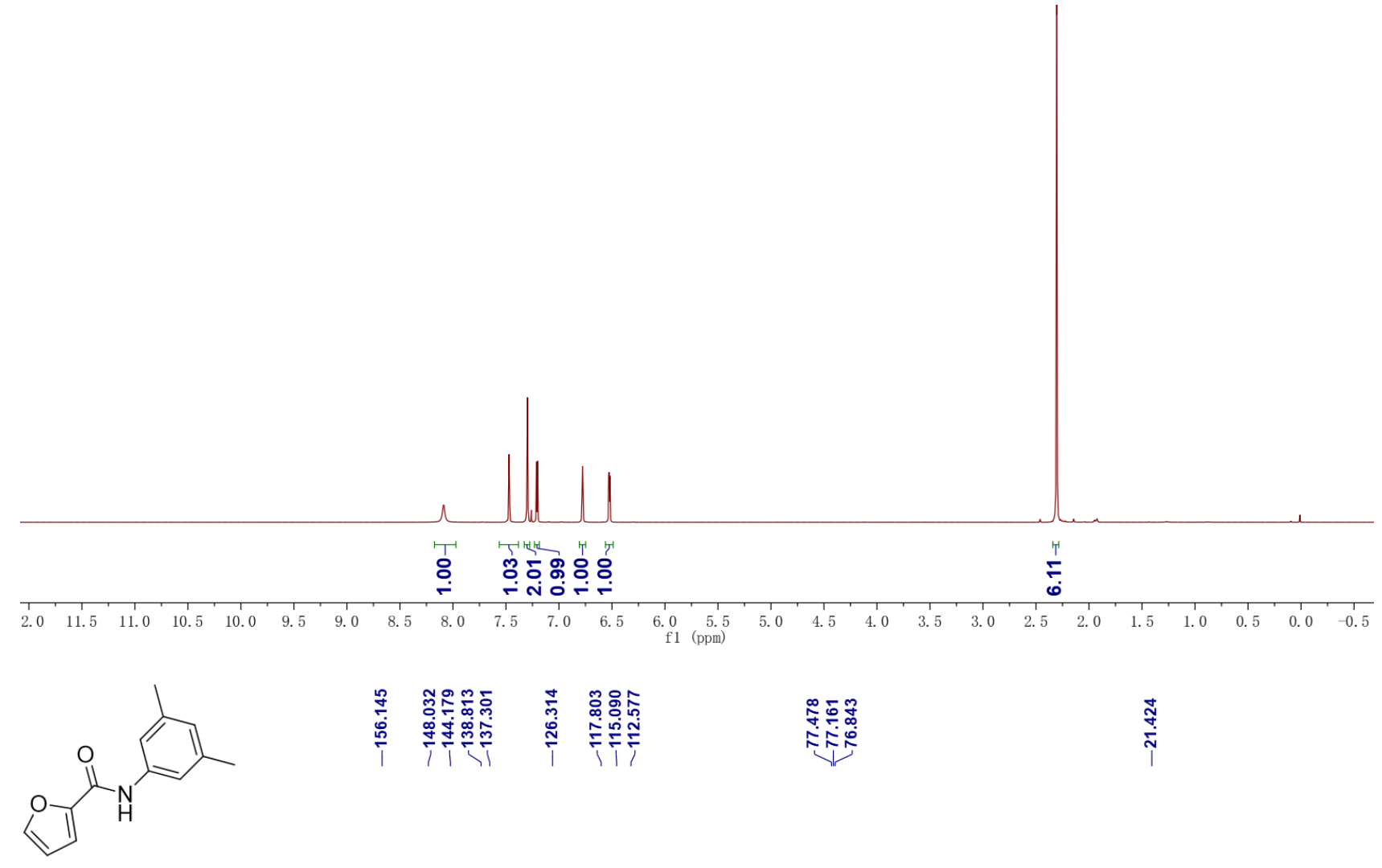

${ }^{13} \mathrm{C}$ NMR $\left(100 \mathrm{MHz}, \mathrm{CDCl}_{3}\right)$

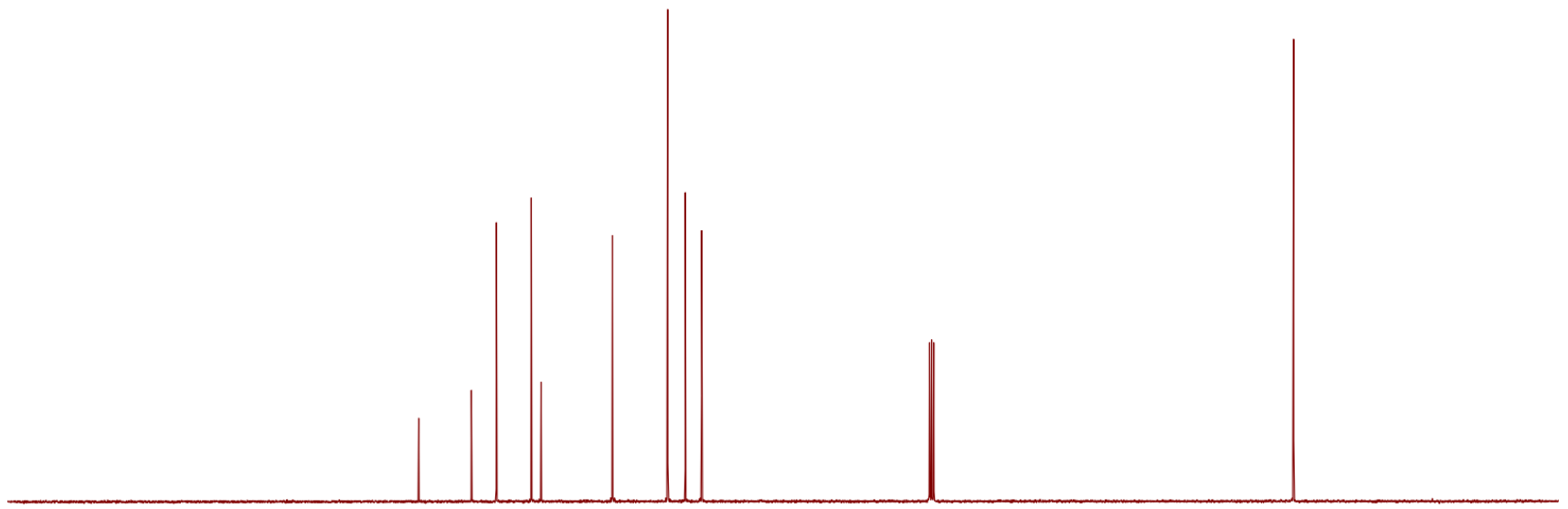

$\begin{array}{llllllllllll}210 & 200 & 190 & 180 & 170 & 160 & 150 & 140 & 130 & 120 & 110 & 100 \\ \mathrm{f} 1(\mathrm{pp}\end{array}$

Figure S54. ${ }^{1} \mathrm{H}$ NMR and ${ }^{13} \mathrm{C}\left\{{ }^{1} \mathrm{H}\right\}$ NMR Spectra of $N$-(3,5-dimethylphenyl)furan-2-carboxamide (5h). 


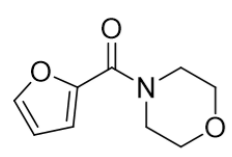

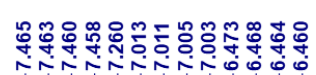

${ }^{1} \mathrm{H} \mathrm{NMR}\left(400 \mathrm{MHz}, \mathrm{CDCl}_{3}\right)$

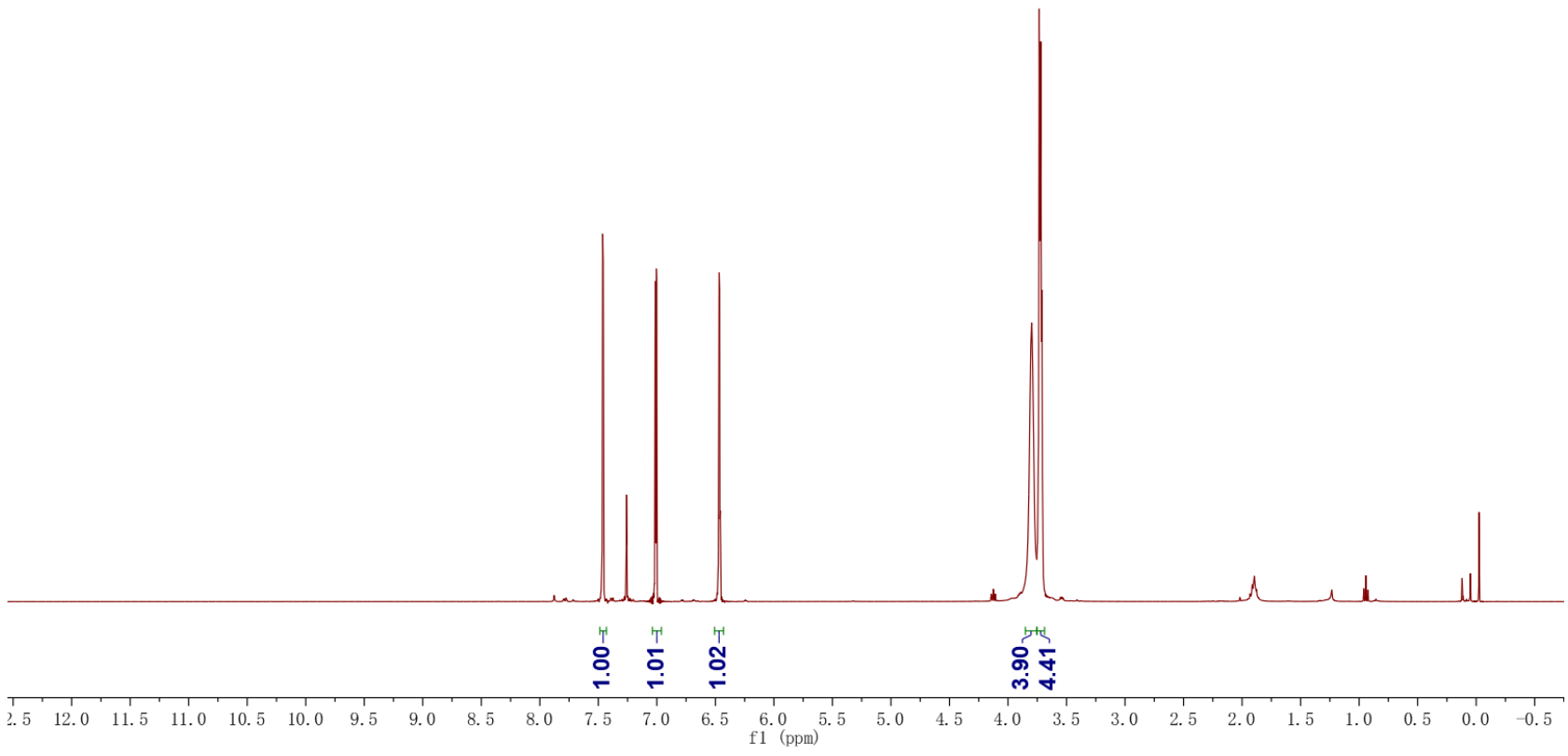<smiles>O=C(c1ccco1)N1CCOCC1</smiles>

${ }^{13} \mathrm{C}$ NMR $\left(100 \mathrm{MHz}, \mathrm{CDCl}_{3}\right)$

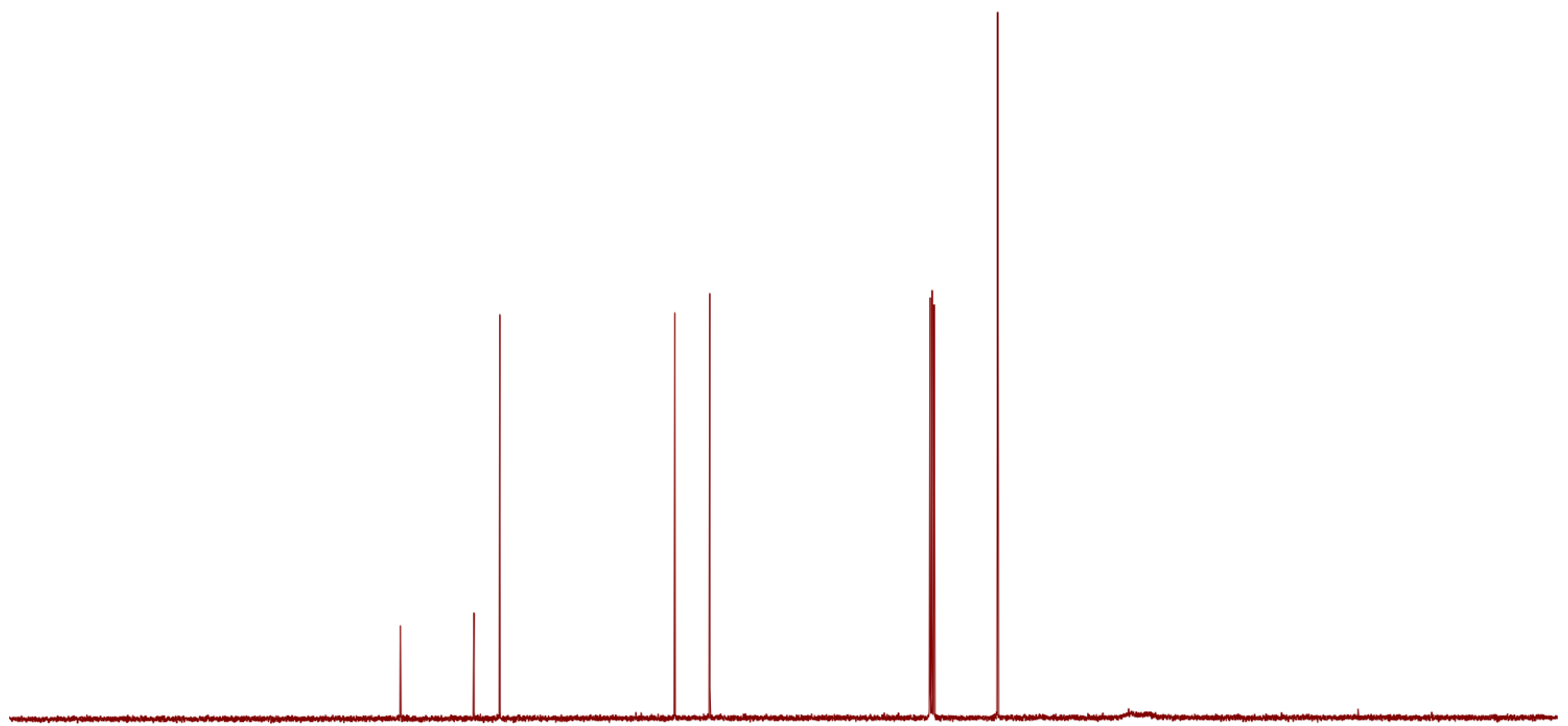

Figure S55. ${ }^{1} \mathrm{H}$ NMR and ${ }^{13} \mathrm{C}\left\{{ }^{1} \mathrm{H}\right\}$ NMR Spectra of furan-2-yl(morpholino)methanone (5i). 
$\overbrace{N}$

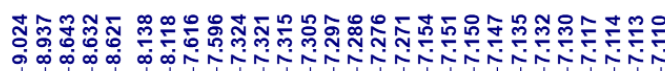

${ }^{1} \mathrm{H}$ NMR (400 MHz, $\mathrm{CDCl}_{3}$ )

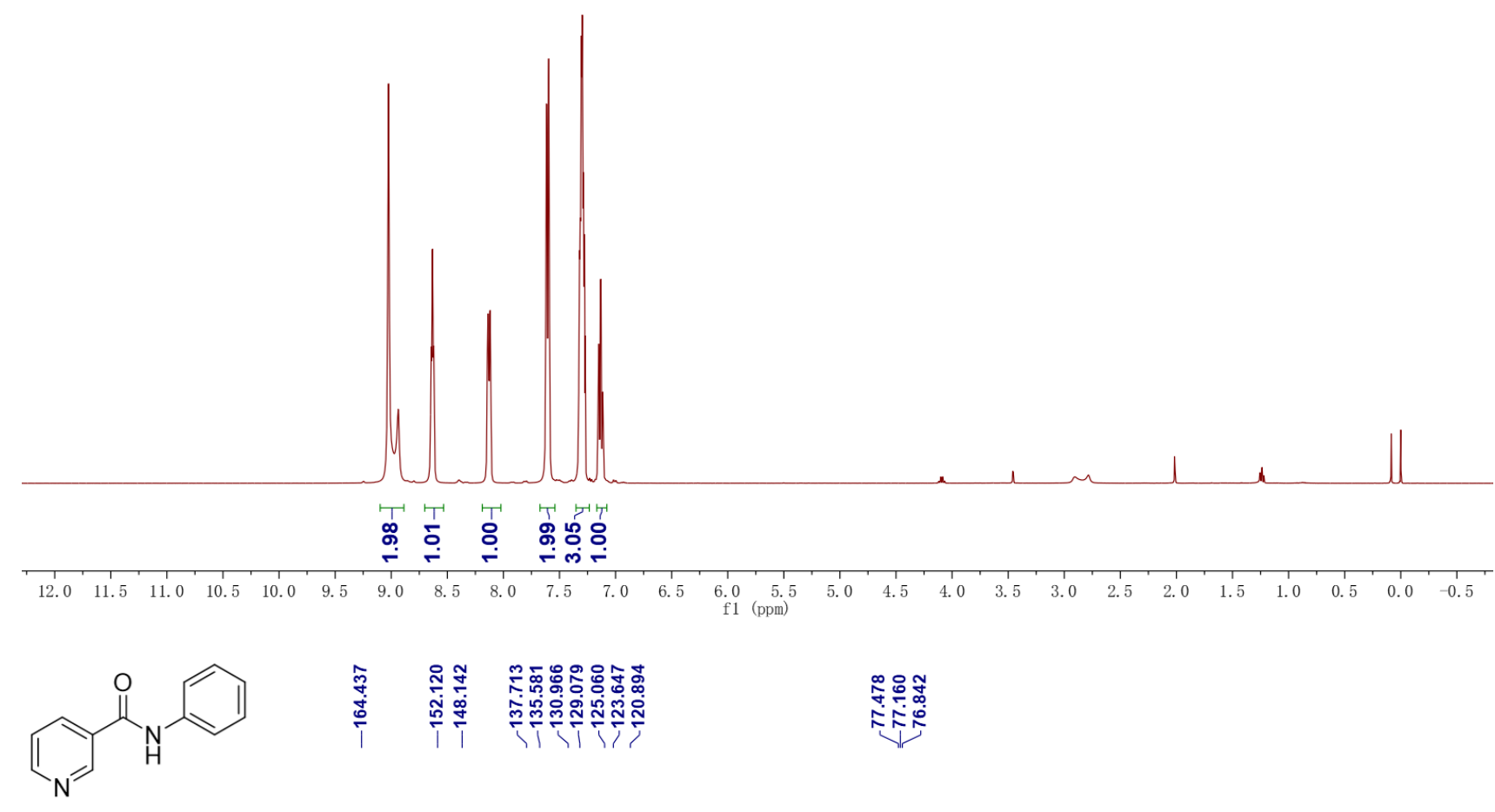

${ }^{13} \mathrm{C}$ NMR (100 MHz, $\mathrm{CDCl}_{3}$ )

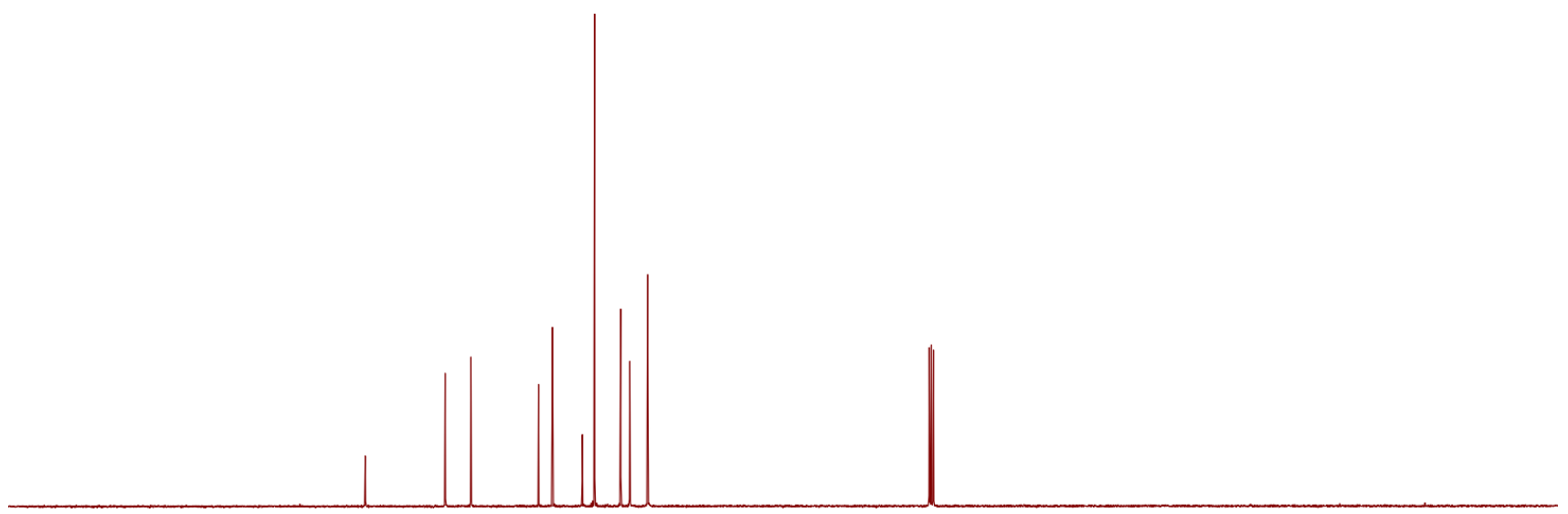

$\begin{array}{lllll}1 & 1 & 1 & 1\end{array}$

$\stackrel{100}{\mathrm{f} 1}(\mathrm{ppm})$

Figure S56. ${ }^{1} \mathrm{H}$ NMR and ${ }^{13} \mathrm{C}\left\{{ }^{1} \mathrm{H}\right\}$ NMR Spectra of $N$-phenylnicotinamide (5j). 
${ }_{N}$

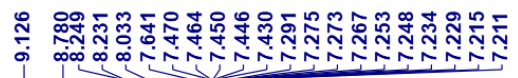

${ }^{1} \mathrm{H}$ NMR $\left(400 \mathrm{MHz}, \mathrm{CDCl}_{3}\right)$

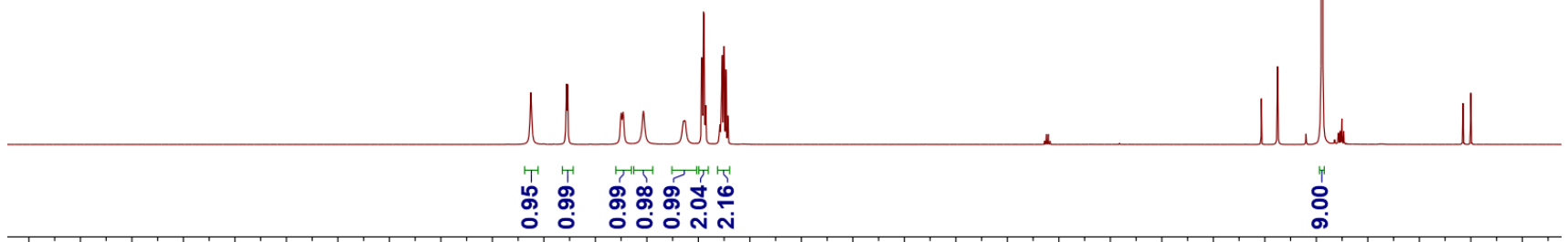

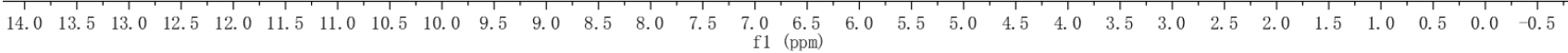<smiles>CC(C)(C)c1ccccc1NC(=O)c1cccnc1</smiles>

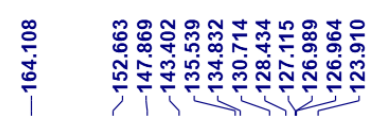

起迸:

${ }^{13} \mathrm{C}$ NMR $\left(100 \mathrm{MHz}, \mathrm{CDCl}_{3}\right)$

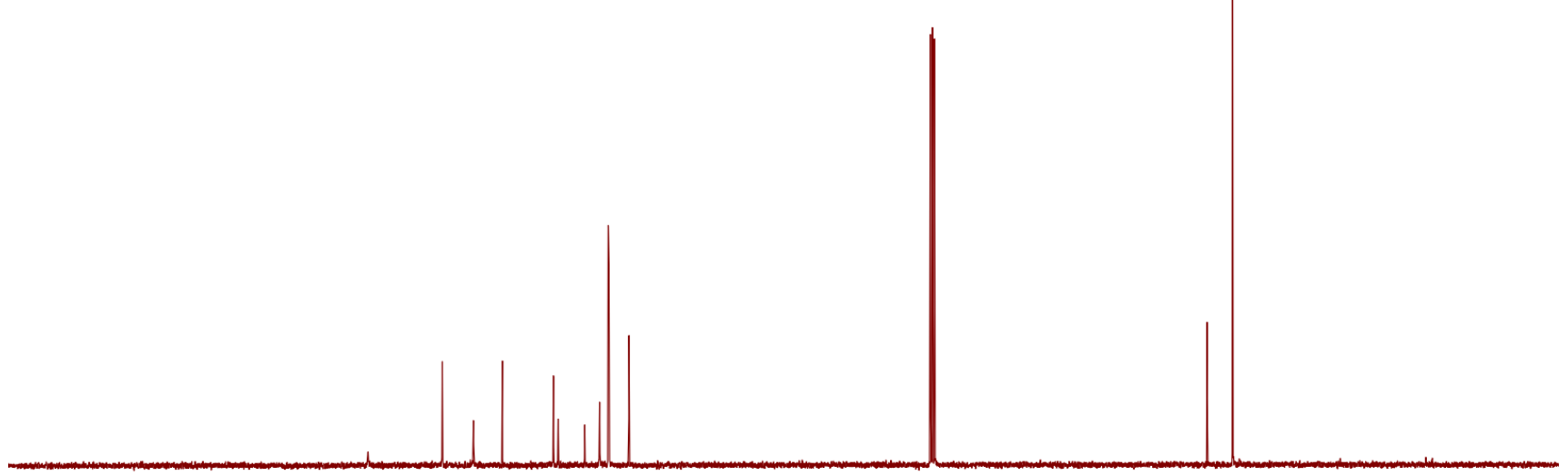

Figure S57. ${ }^{1} \mathrm{H}$ NMR and ${ }^{13} \mathrm{C}\left\{{ }^{1} \mathrm{H}\right\}$ NMR Spectra of $N$-(2-Isopropylphenyl)nicotinamide (5k). 
<smiles>Cc1cc(NC(=O)c2cccnc2)cc(C)c1C</smiles>

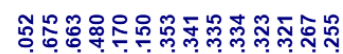

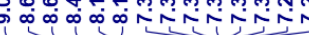

${ }^{1} \mathrm{H}$ NMR (400 MHz, $\left.\mathrm{CDCl}_{3}\right)$

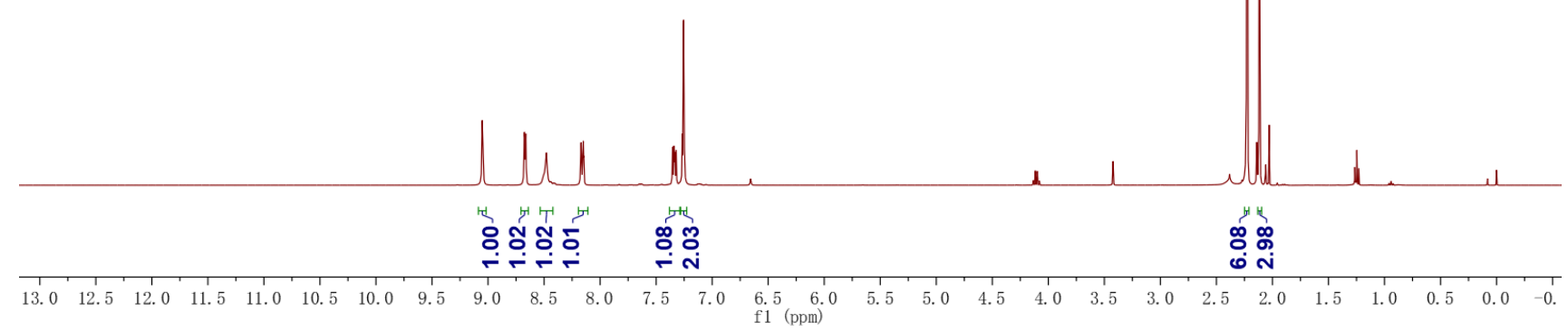<smiles>Cc1cc(NC(=O)c2cccnc2)cc(C)c1C</smiles>

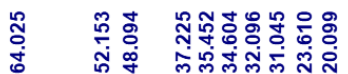

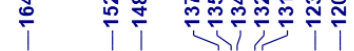

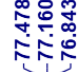

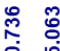

$N^{\prime}$

${ }^{13} \mathrm{C} \mathrm{NMR}\left(100 \mathrm{MHz}, \mathrm{CDCl}_{3}\right)$

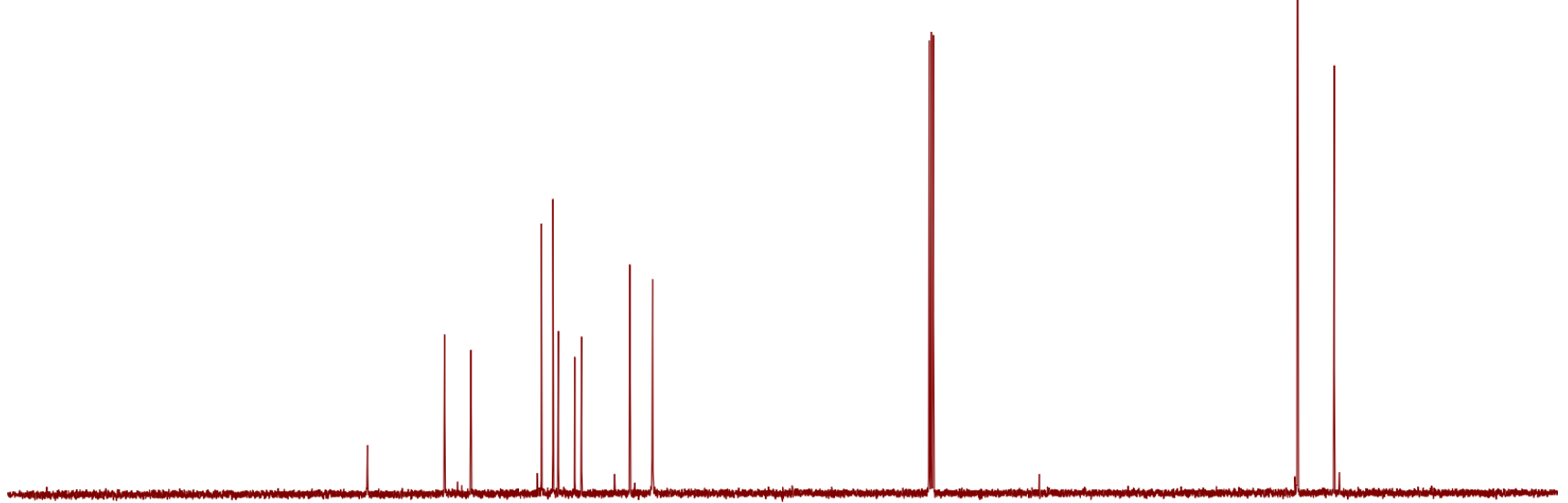

Figure S58. ${ }^{1} \mathrm{H}$ NMR and ${ }^{13} \mathrm{C}\left\{{ }^{1} \mathrm{H}\right\}$ NMR Spectra of $N$-(3,4,5-trimethylphenyl)nicotinamide (5l). 


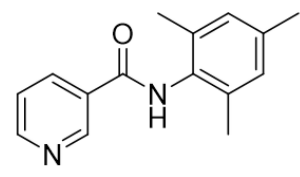

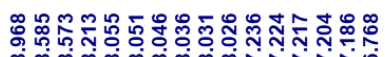

${ }^{1} \mathrm{H} \mathrm{NMR}\left(400 \mathrm{MHz}, \mathrm{CDCl}_{3}\right)$

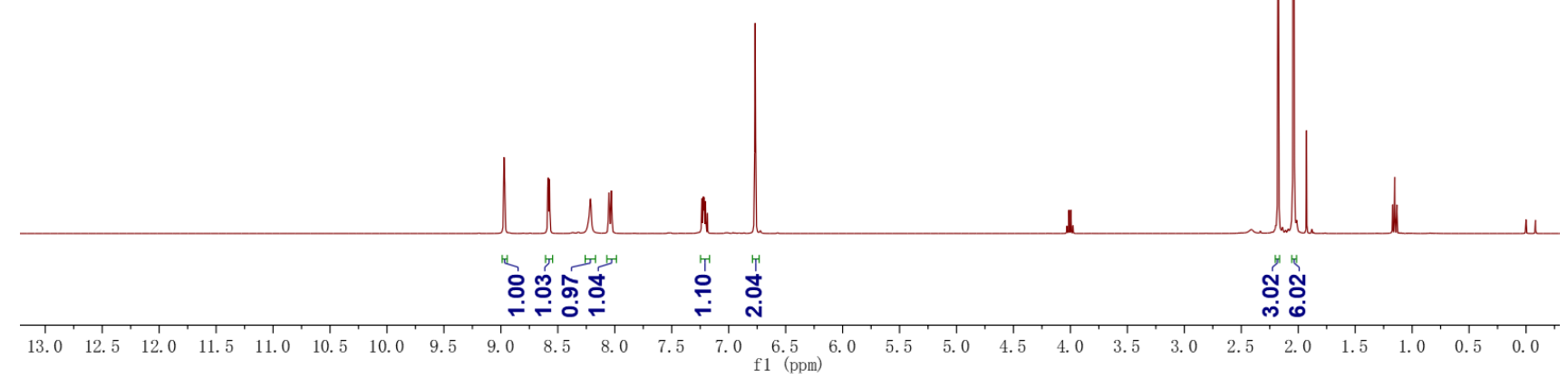<smiles>Cc1cc(C)c(NC(=O)c2cccnc2)c(C)c1</smiles>

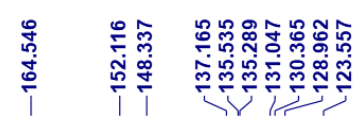

${ }^{13} \mathrm{C}$ NMR $\left(100 \mathrm{MHz}, \mathrm{CDCl}_{3}\right)$

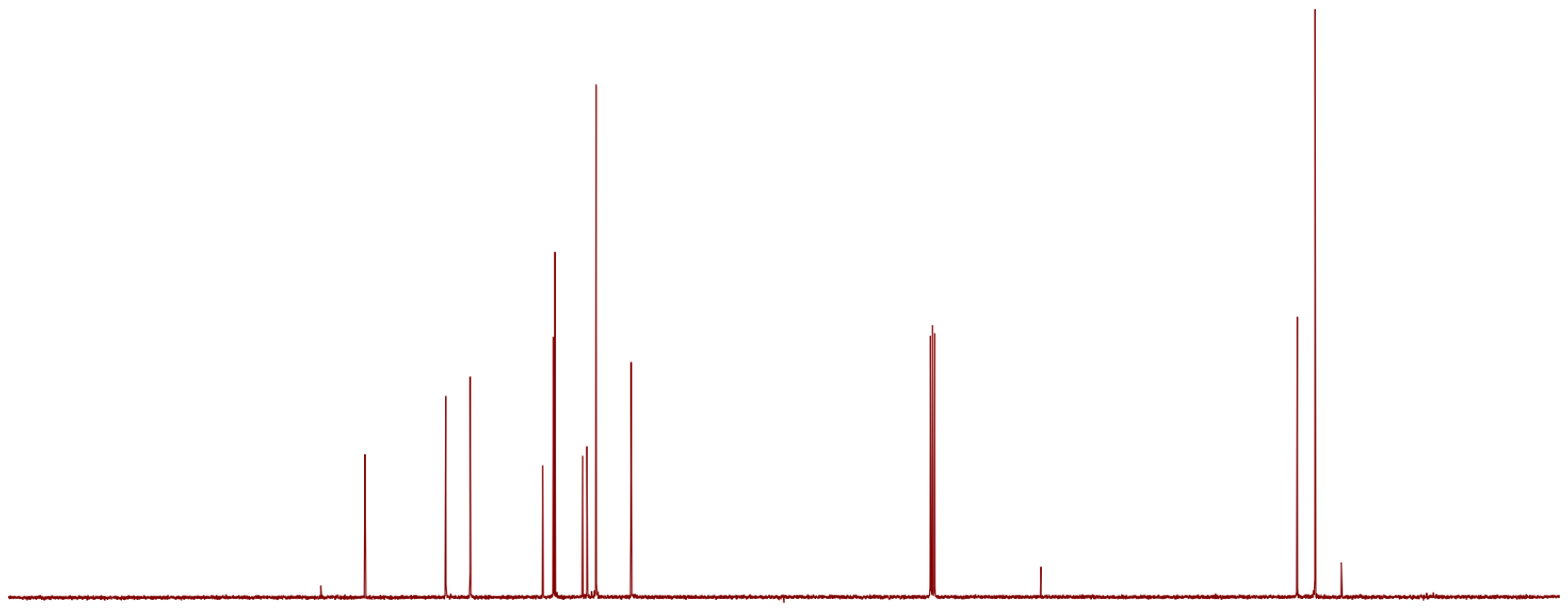

Figure S59. ${ }^{1} \mathrm{H}$ NMR and ${ }^{13} \mathrm{C}\left\{{ }^{1} \mathrm{H}\right\}$ NMR Spectra of $N$-mesitylnicotinamide (5m). 
<smiles>CCCCc1cccc(C(C)C)c1NC(=O)c1cccnc1</smiles>

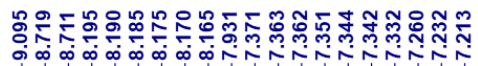

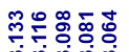

in minim

产

${ }^{1} \mathrm{H} \mathrm{NMR}\left(400 \mathrm{MHz}, \mathrm{CDCl}_{3}\right)$

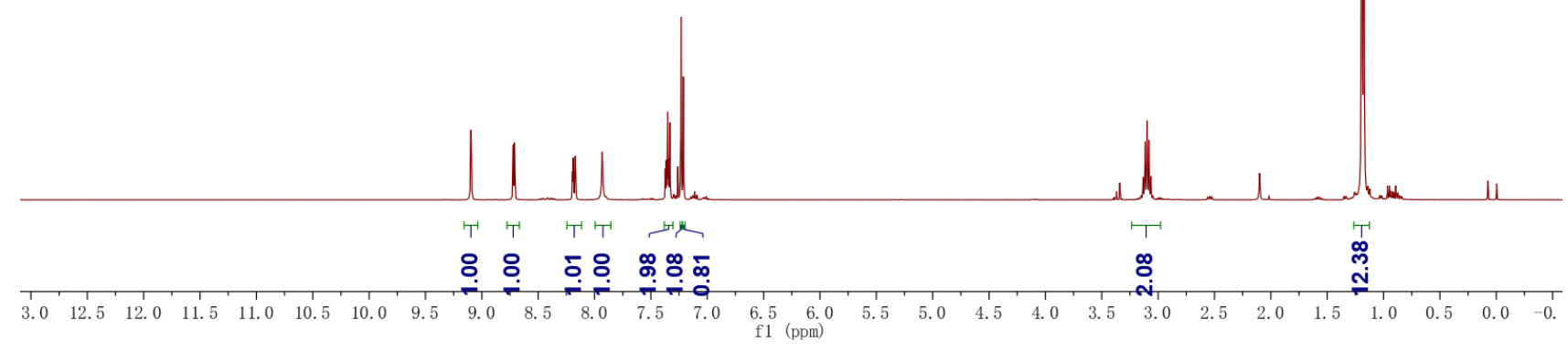<smiles>CCCc1cccc(C(C)C)c1NC(=O)c1cccnc1</smiles>

\section{营}

实产产

㪘

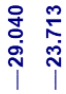

${ }^{13} \mathrm{C}$ NMR (100 MHz, $\left.\mathrm{CDCl}_{3}\right)$

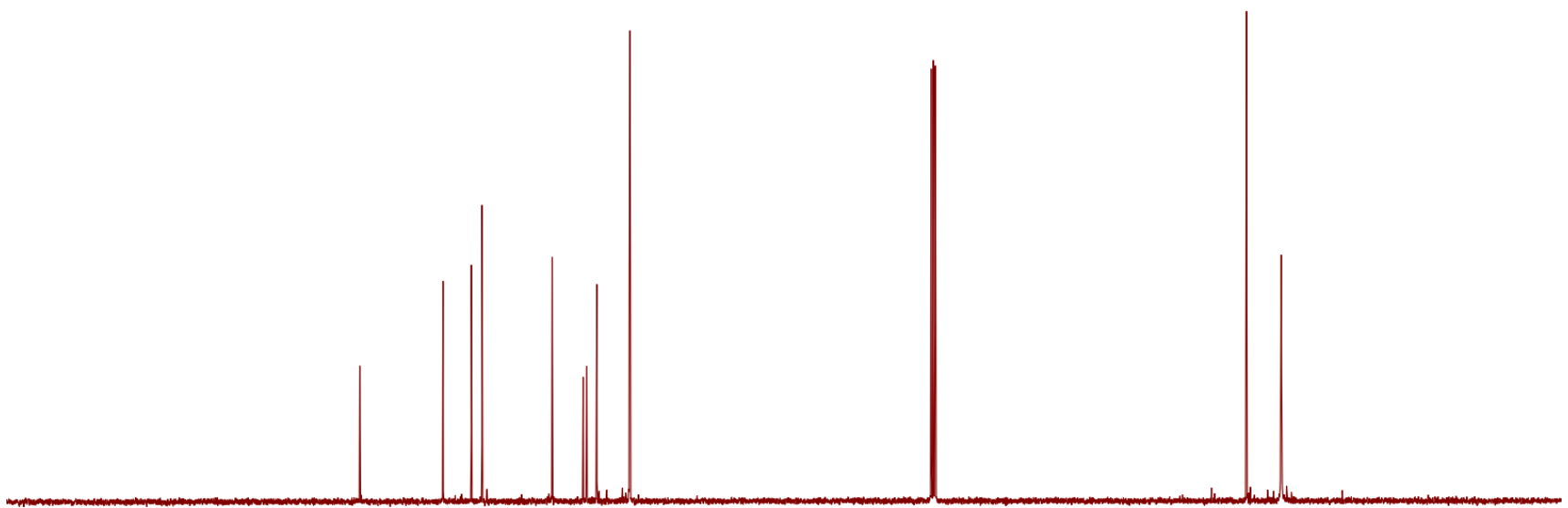

$\begin{array}{llll}210 & 200 & 190 & 180\end{array}$

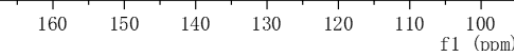

90

70

1
60
50

$40 \quad 30$

Figure S60. ${ }^{1} \mathrm{H}$ NMR and ${ }^{13} \mathrm{C}\left\{{ }^{1} \mathrm{H}\right\}$ NMR Spectra of $N$-(2,6-diisopropylphenyl)nicotinamide (5n). 


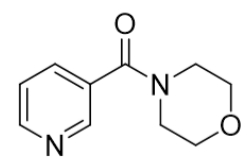

${ }^{1} \mathrm{H} \mathrm{NMR}\left(400 \mathrm{MHz}, \mathrm{CDCl}_{3}\right)$

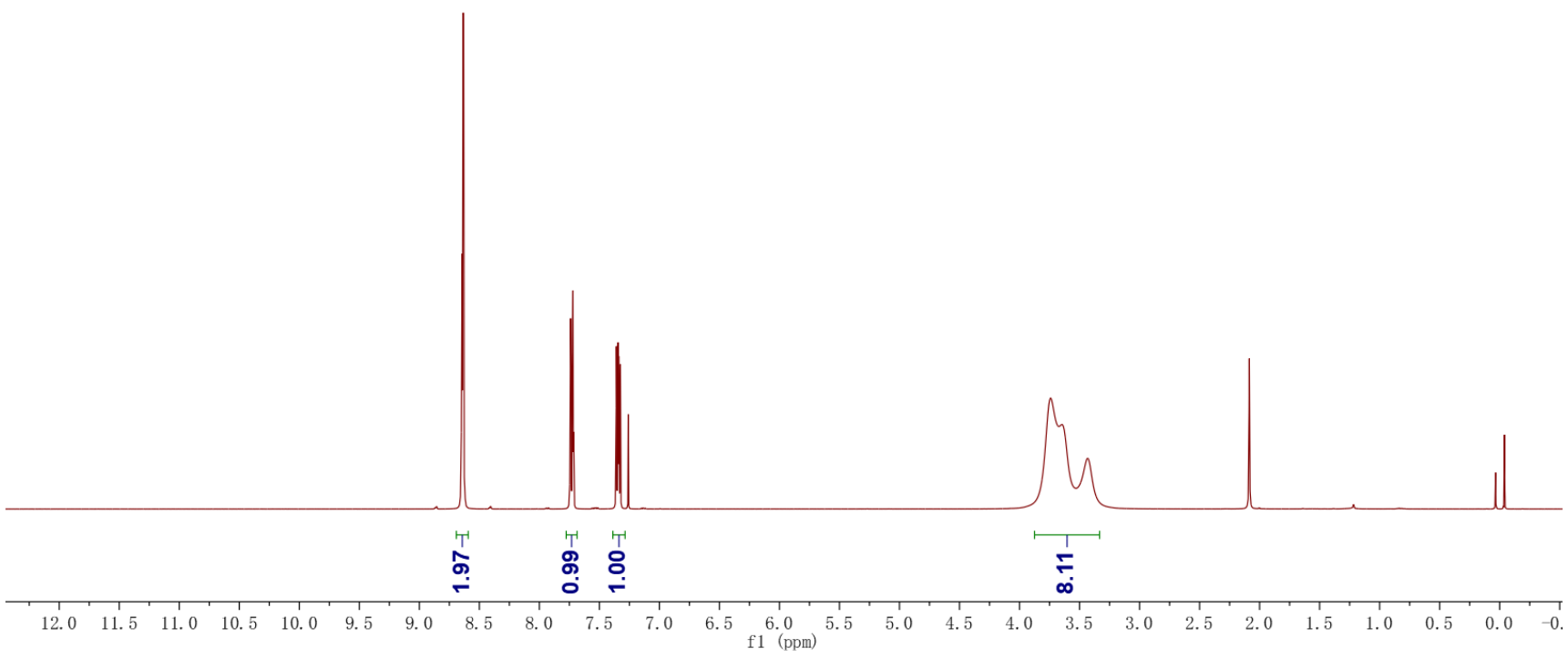

年

${ }^{13} \mathrm{C}$ NMR $\left(100 \mathrm{MHz}, \mathrm{CDCl}_{3}\right)$

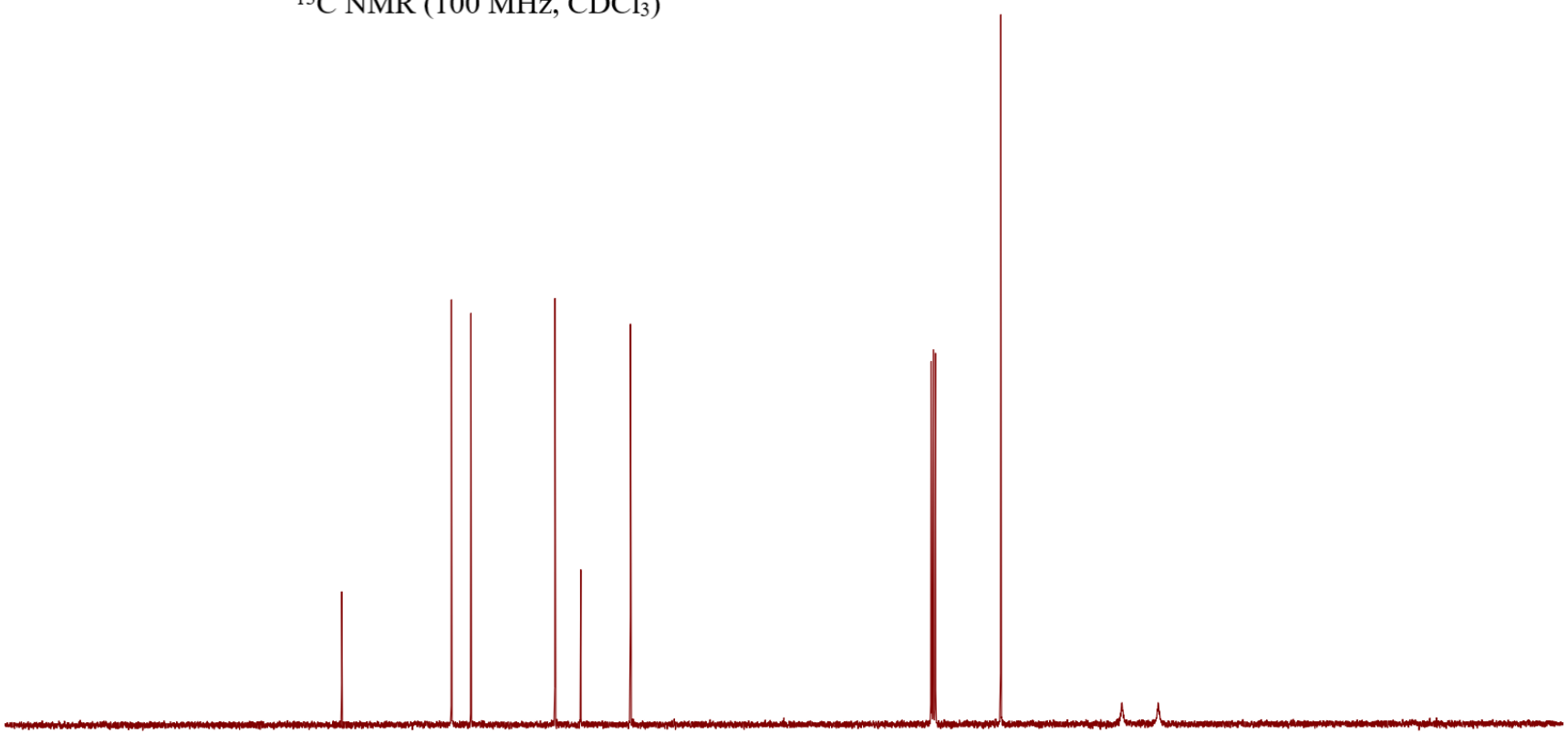

Figure S61. ${ }^{1} \mathrm{H}$ NMR and ${ }^{13} \mathrm{C}\left\{{ }^{1} \mathrm{H}\right\}$ NMR Spectra of Morpholino(pyridin-3-yl)methanone (5o). 


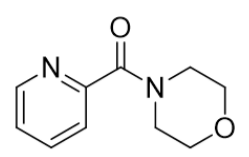

${ }^{1} \mathrm{H}$ NMR $\left(400 \mathrm{MHz}, \mathrm{CDCl}_{3}\right)$

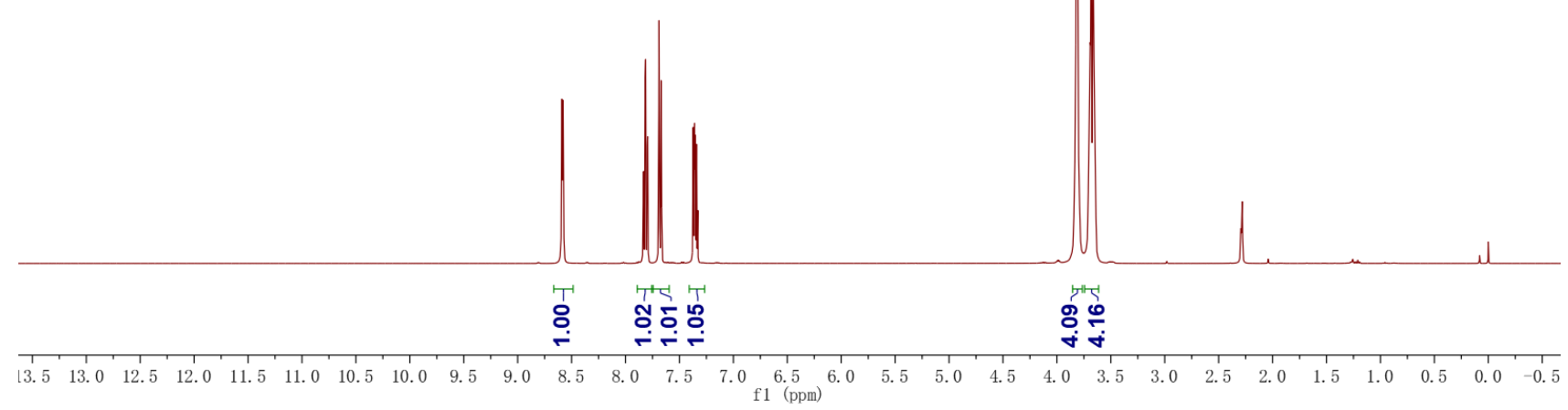

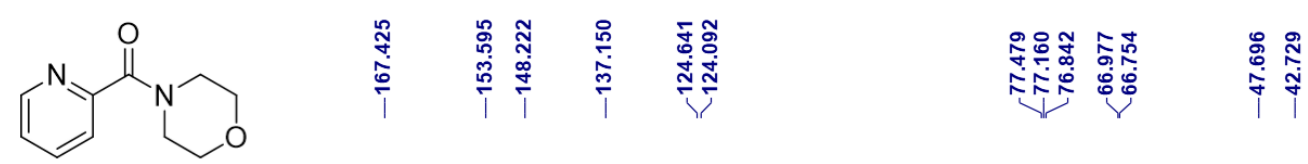

${ }^{13} \mathrm{C} \mathrm{NMR}\left(100 \mathrm{MHz}, \mathrm{CDCl}_{3}\right)$

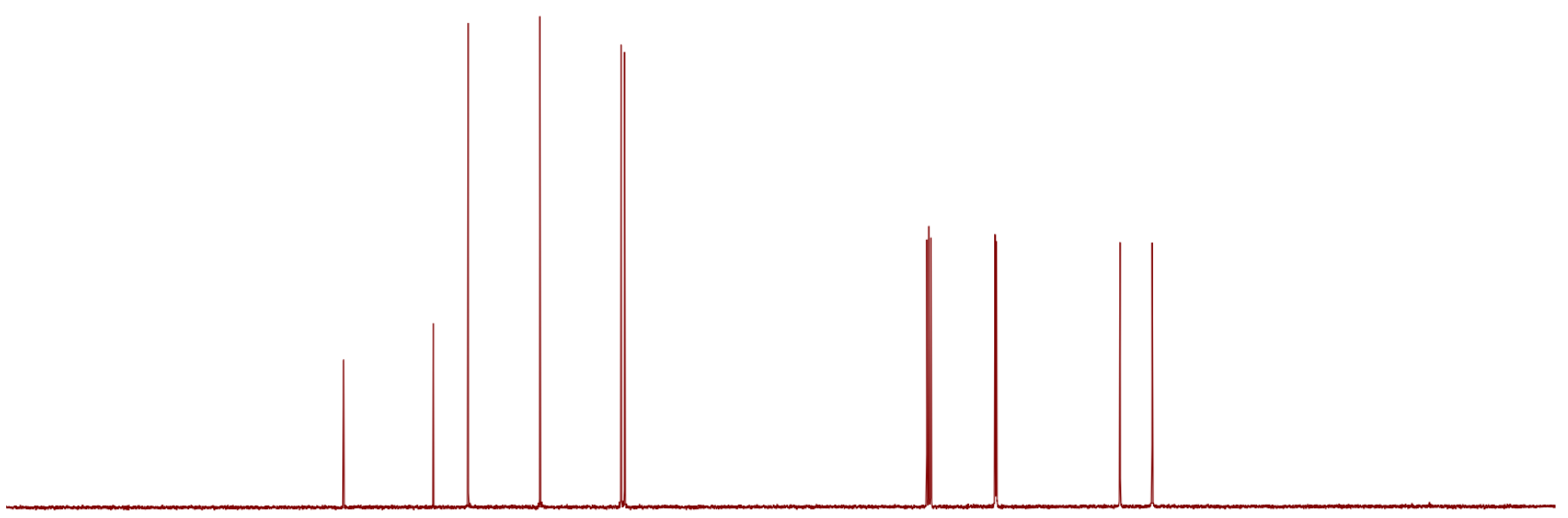

$\begin{array}{llllllllllll}210 & 200 & 190 & 180 & 170 & 160 & 150 & 140 & 130 & 120 & 110 & \begin{array}{l}100 \\ \mathrm{f} 1\end{array}(\mathrm{ppm})\end{array}$

Figure S62. ${ }^{1} \mathrm{H}$ NMR and ${ }^{13} \mathrm{C}\left\{{ }^{1} \mathrm{H}\right\}$ NMR Spectra of Morpholino(pyridin-2-yl)methanone (5p). 
<smiles>Cc1cnc(C(=O)N2CCOCC2)cn1</smiles>

${ }^{1} \mathrm{H} \mathrm{NMR}\left(400 \mathrm{MHz}, \mathrm{CDCl}_{3}\right)$

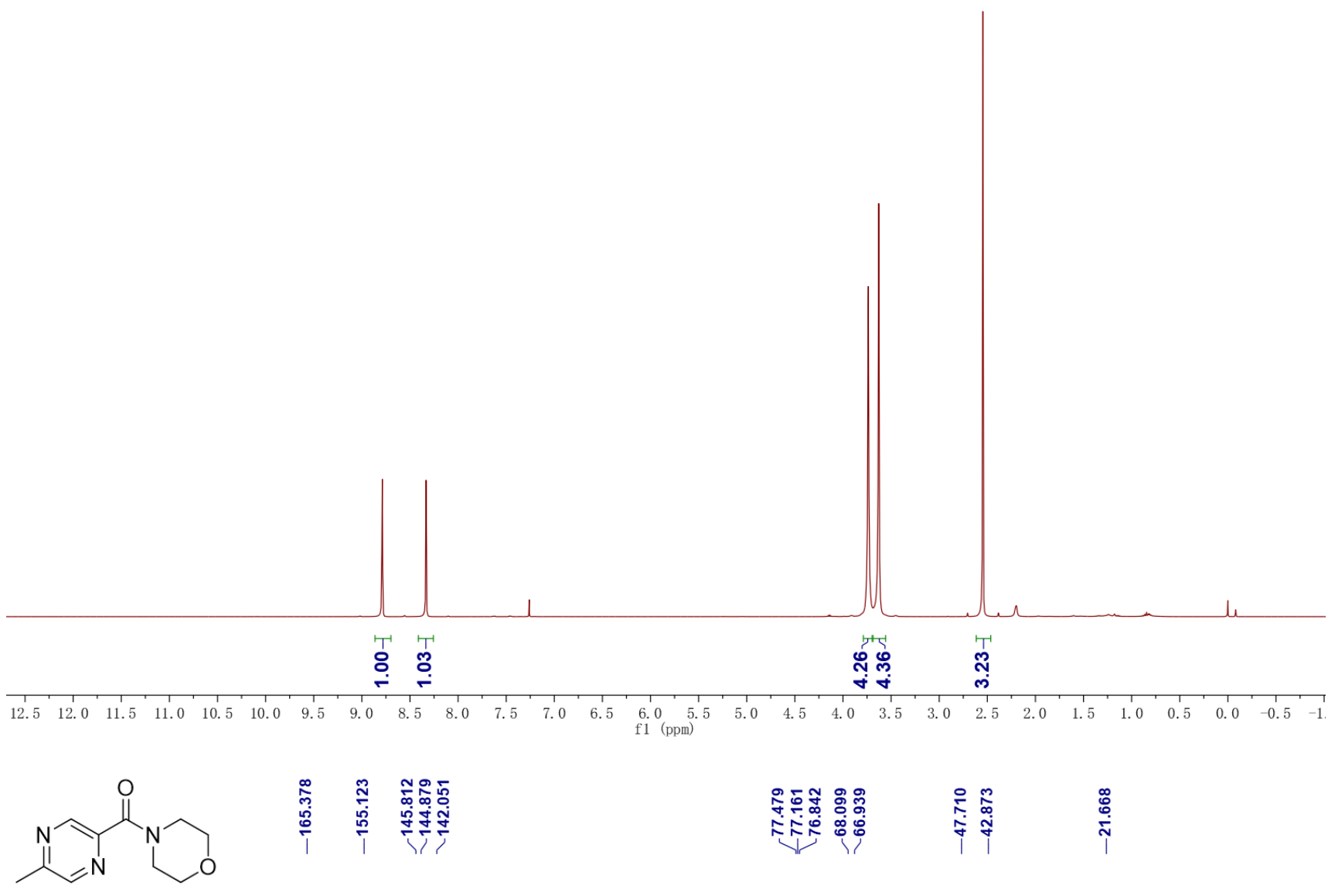

${ }^{13} \mathrm{C} \mathrm{NMR}\left(100 \mathrm{MHz}, \mathrm{CDCl}_{3}\right)$

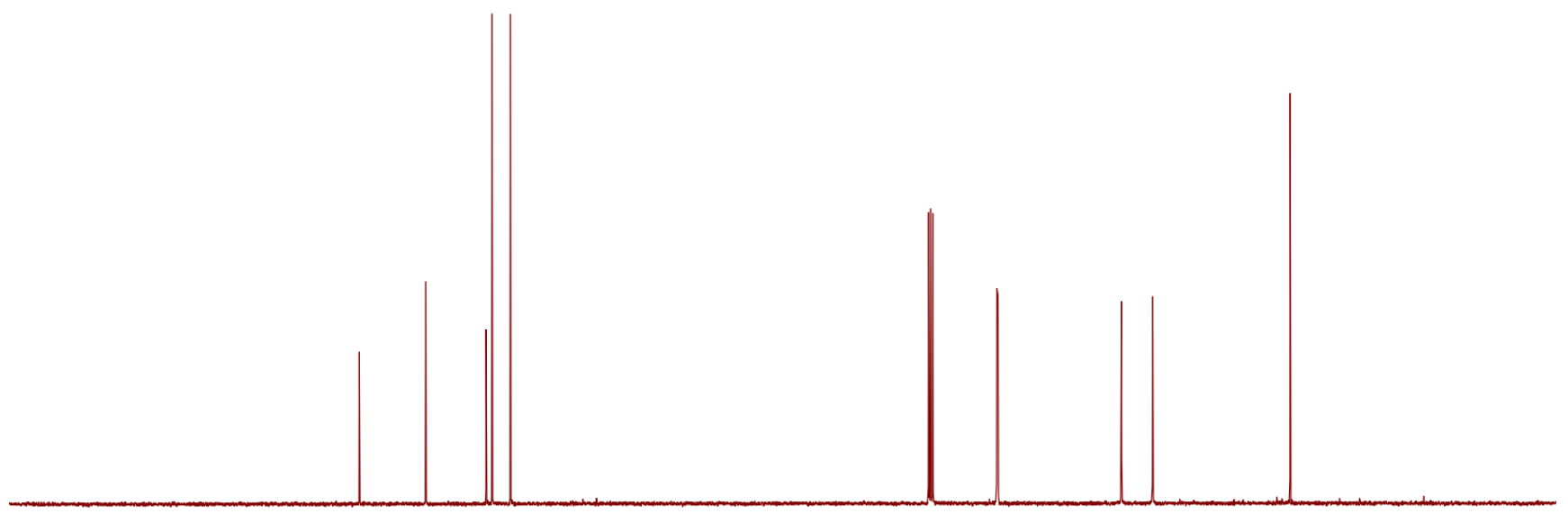

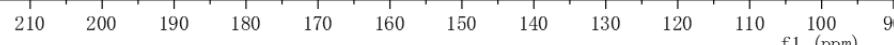

Figure S63. ${ }^{1} \mathrm{H}$ NMR and ${ }^{13} \mathrm{C}\left\{{ }^{1} \mathrm{H}\right\}$ NMR Spectra of (5-methylpyrazin-2-yl)(morpholino)methanone (5q). 


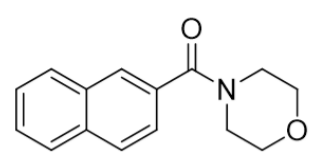

LO

${ }^{1} \mathrm{H} \mathrm{NMR}\left(400 \mathrm{MHz}, \mathrm{CDCl}_{3}\right)$

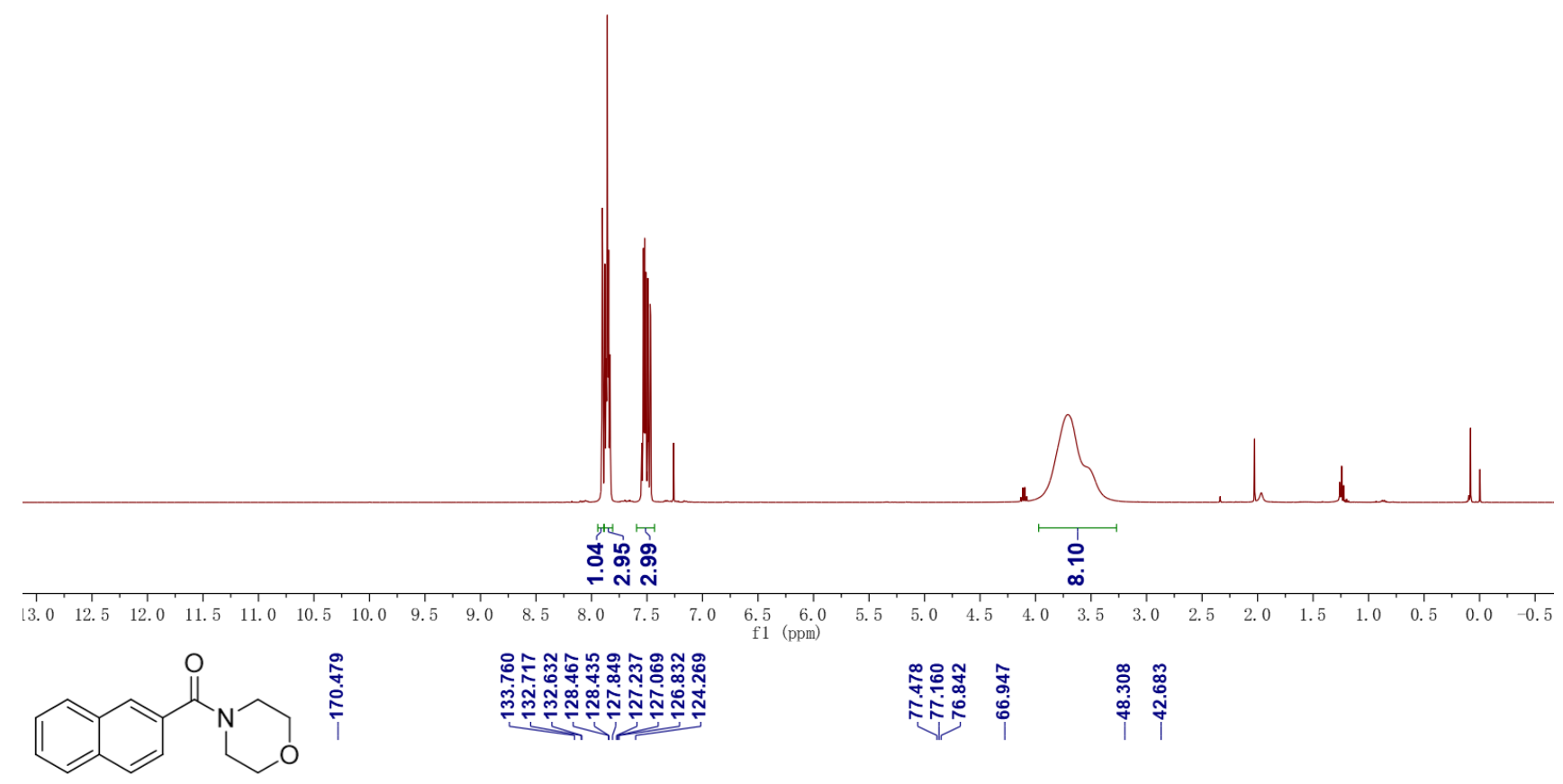

${ }^{13} \mathrm{C}$ NMR $\left(100 \mathrm{MHz}, \mathrm{CDCl}_{3}\right)$

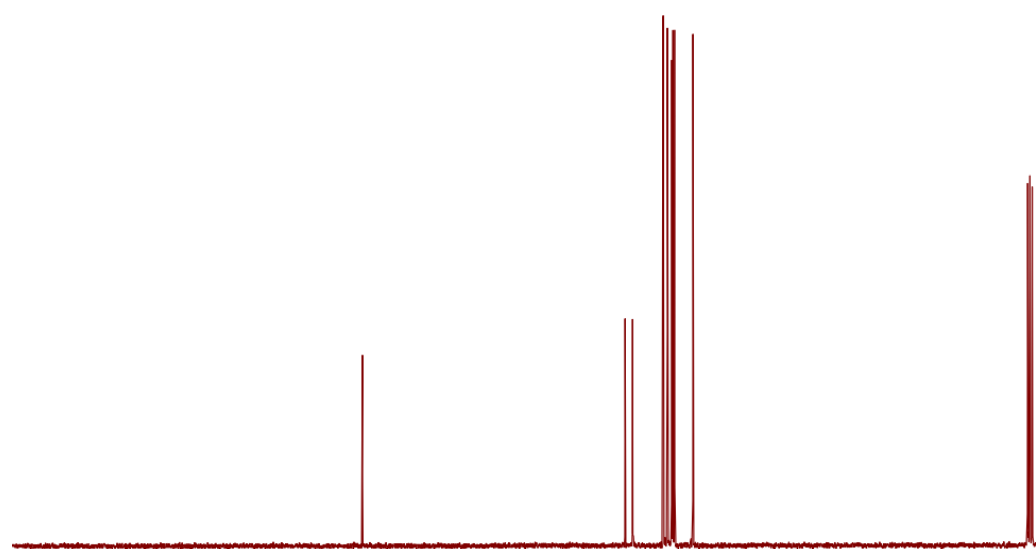

$\begin{array}{llllllllllll}210 & 200 & 190 & 180 & 170 & 160 & 150 & 140 & 130 & 120 & 110 & 100 \\ \mathrm{f} 1 & (\mathrm{ppm})\end{array}$

Figure S64. ${ }^{1} \mathrm{H}$ NMR and ${ }^{13} \mathrm{C}\left\{{ }^{1} \mathrm{H}\right\}$ NMR Spectra of morpholino(naphthalen-2-yl)methanone (5r). 


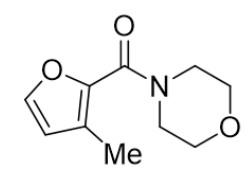

$\stackrel{i}{i} \quad \underset{\substack{\text { I } \\ i}}{i}$



$\stackrel{\leftrightarrow}{\stackrel{2}{i ் ~}}$

${ }^{1} \mathrm{H} \mathrm{NMR}\left(400 \mathrm{MHz}, \mathrm{CDCl}_{3}\right)$

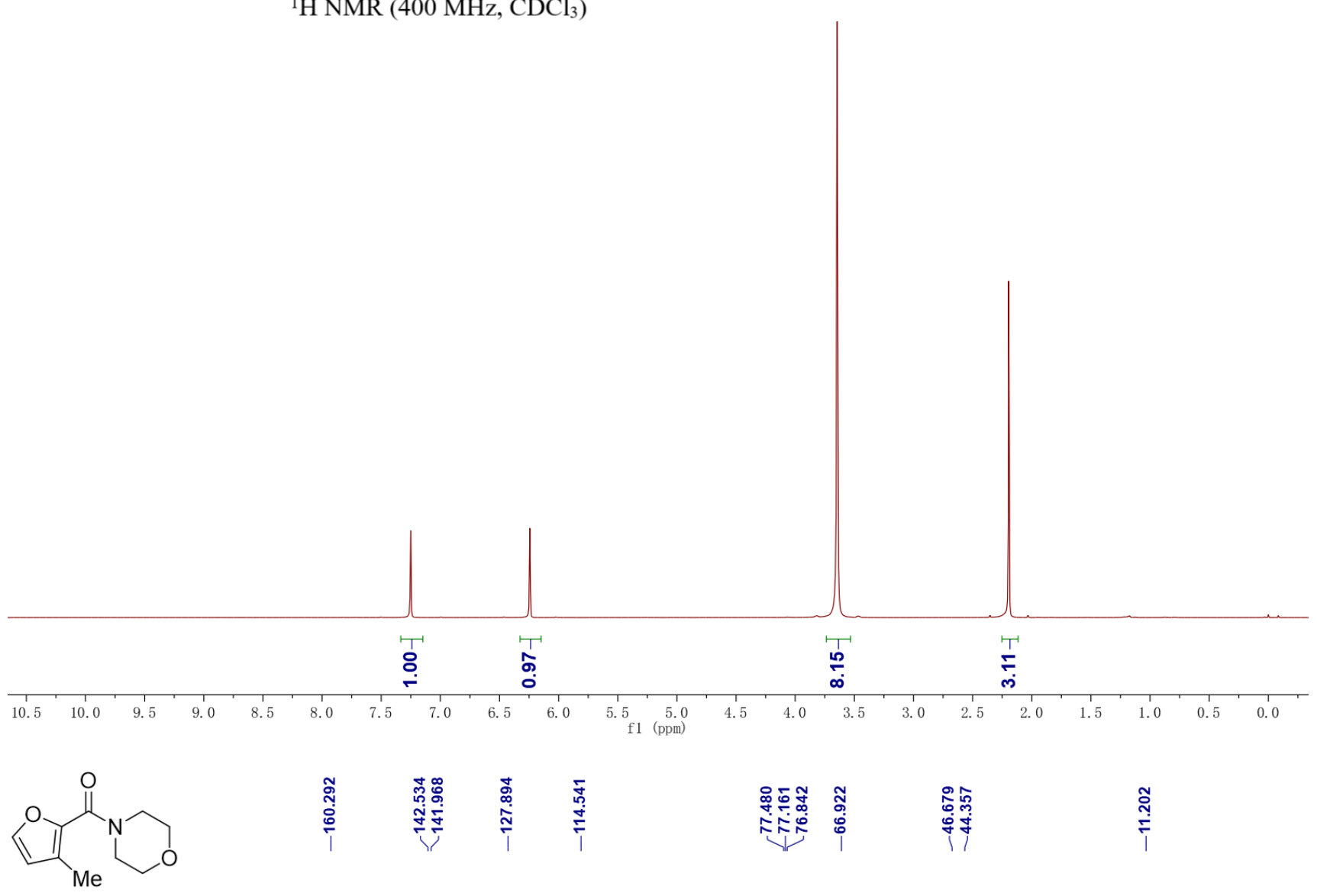

$\left.{ }^{13} \mathrm{C} \mathrm{NMR} \mathrm{(100} \mathrm{MHz,} \mathrm{CDCl}_{3}\right)$

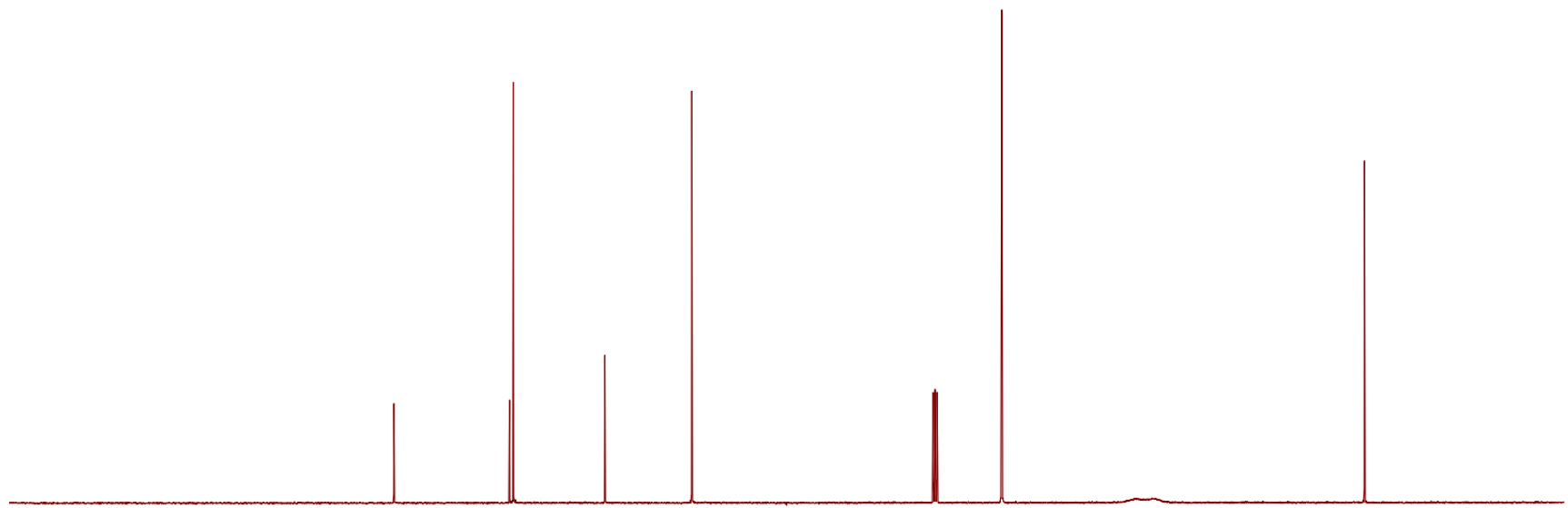

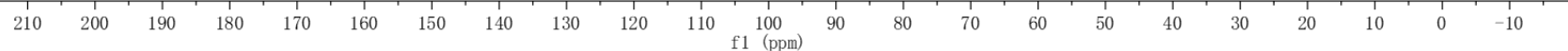

Figure S65. ${ }^{1} \mathrm{H}$ NMR and ${ }^{13} \mathrm{C}\left\{{ }^{1} \mathrm{H}\right\}$ NMR Spectra of (3-methylfuran-2-yl)(morpholino)methanone (5s). 


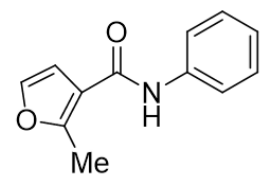

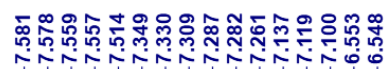

${ }^{1} \mathrm{H} \mathrm{NMR}\left(400 \mathrm{MHz}, \mathrm{CDCl}_{3}\right)$

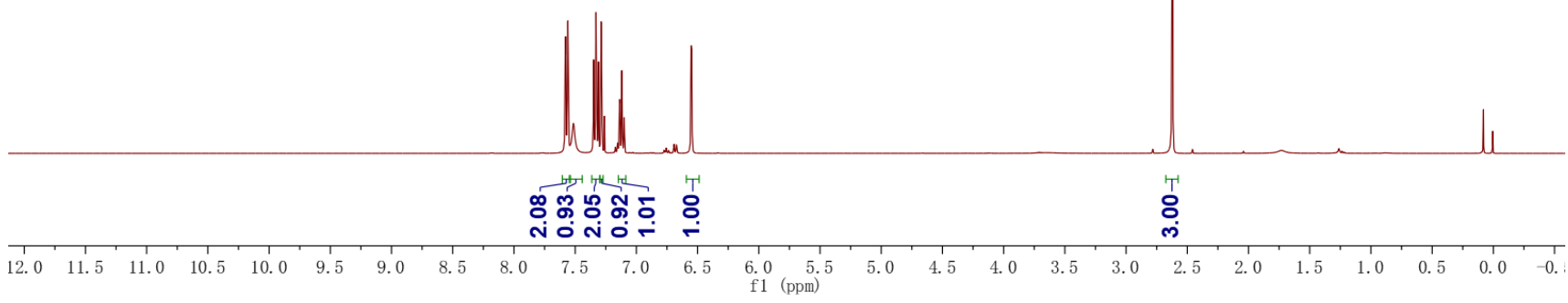<smiles>Cc1occc1C(=O)Nc1ccccc1</smiles>

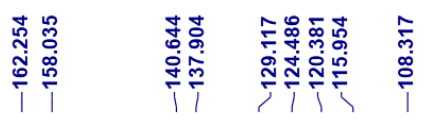

${ }^{13} \mathrm{C} \mathrm{NMR}\left(100 \mathrm{MHz}, \mathrm{CDCl}_{3}\right)$

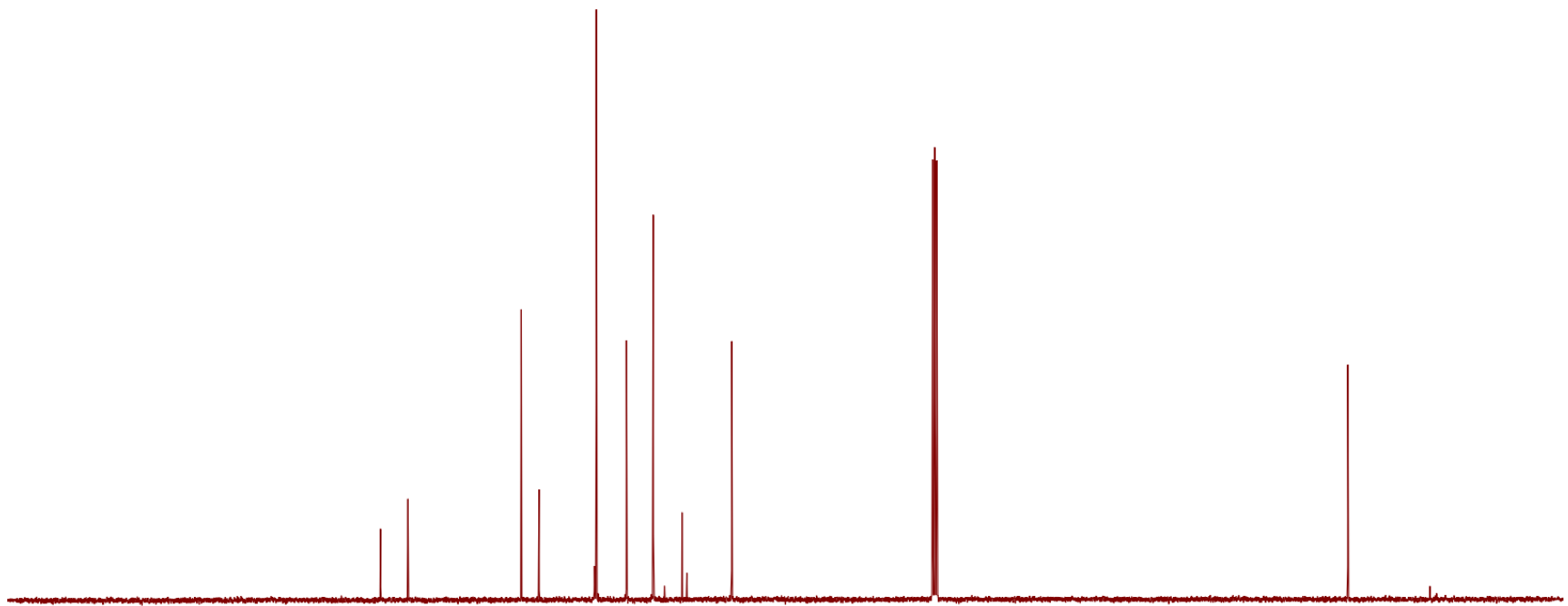

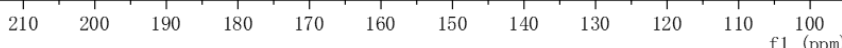

Figure S66. ${ }^{1} \mathrm{H}$ NMR and ${ }^{13} \mathrm{C}\left\{{ }^{1} \mathrm{H}\right\}$ NMR Spectra of Fenfuram $(\mathbf{5 t})$. 


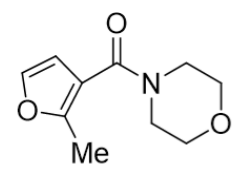

${ }^{1} \mathrm{H} \mathrm{NMR}\left(400 \mathrm{MHz}, \mathrm{CDCl}_{3}\right)$

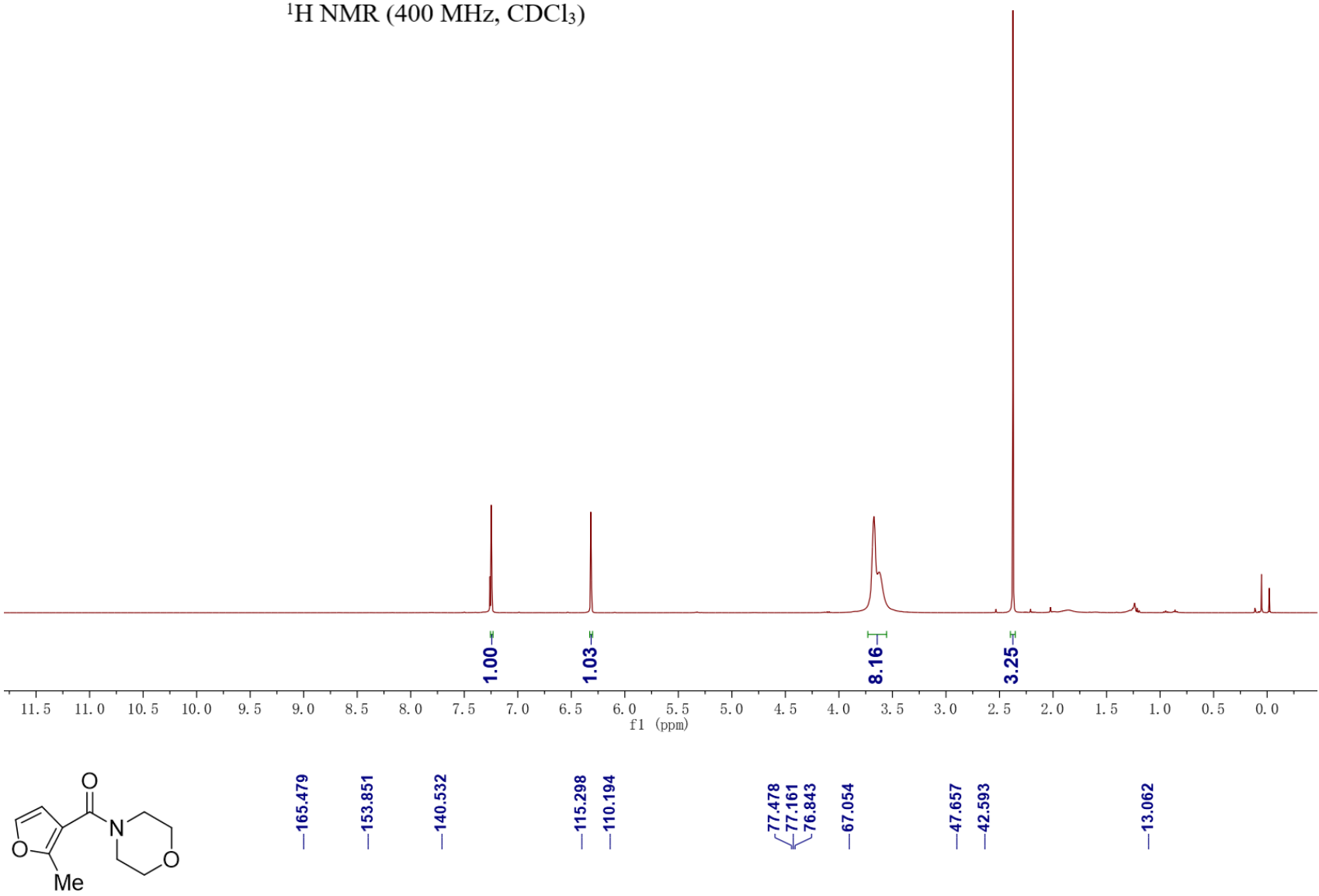

${ }^{13} \mathrm{C}$ NMR $\left(100 \mathrm{MHz}, \mathrm{CDCl}_{3}\right)$

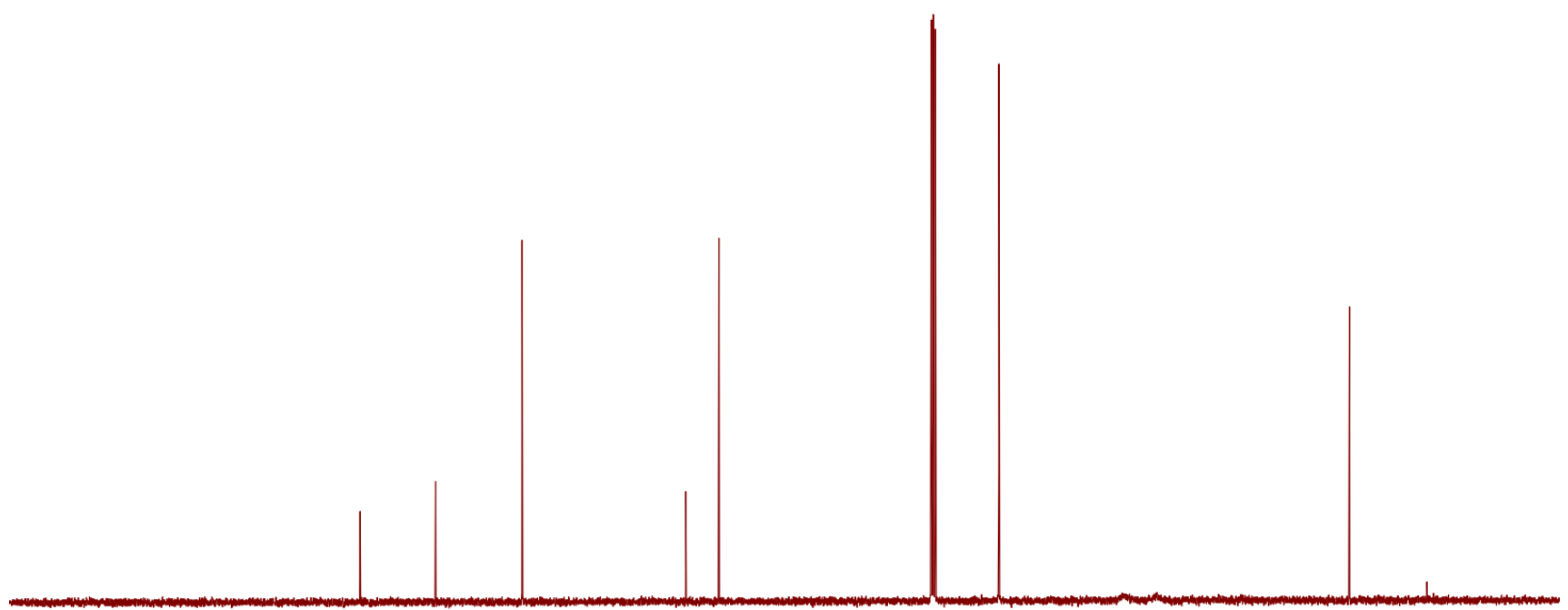

$\begin{array}{lllllllllllllllllllllllll}1 & 1 \\ 210 & 200 & 190 & 180 & 170 & 160 & 150 & 140 & 130 & 120 & 110 & 100 & 90 & 80 & 70 & 60 & 50 & 40 & 30 & 20 & 10 & 0 & -10 & 1\end{array}$

Figure S67. ${ }^{1} \mathrm{H}$ NMR and ${ }^{13} \mathrm{C}\left\{{ }^{1} \mathrm{H}\right\}$ NMR Spectra of 4-(2-methyl-furan-3-carbonyl)-morpholine (5u). 
<smiles>O=C(Nc1ccccc1)c1ccsc1</smiles>

${ }^{1} \mathrm{H}$ NMR (400 MHz, DMSO- $\left.d_{6}\right)$
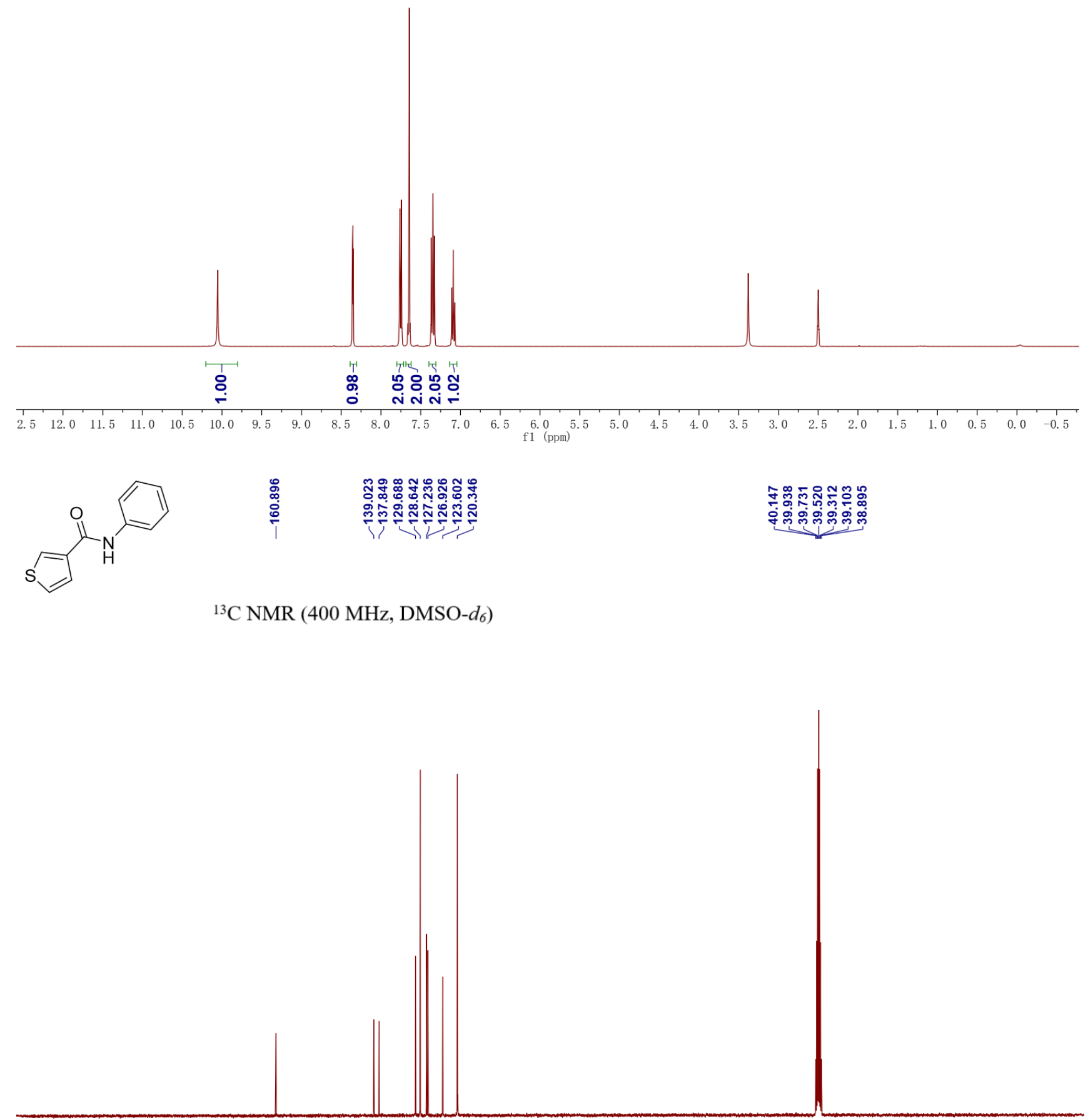

$\begin{array}{llllllll}210 & 200 & 190 & 180 & 170 & 160 & 150 & 140\end{array}$

Figure S68. ${ }^{1} \mathrm{H}$ NMR and ${ }^{13} \mathrm{C}\left\{{ }^{1} \mathrm{H}\right\}$ NMR Spectra of $N$-phenylthiophene-3-carboxamide (5v). 
<smiles>Cc1ocnc1C(=O)Nc1ccccc1</smiles>

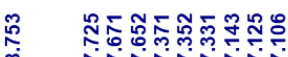 \\ i rin}

$\mathrm{H}$ NMR (400 MHz, $\left.\mathrm{CDCl}_{3}\right)$
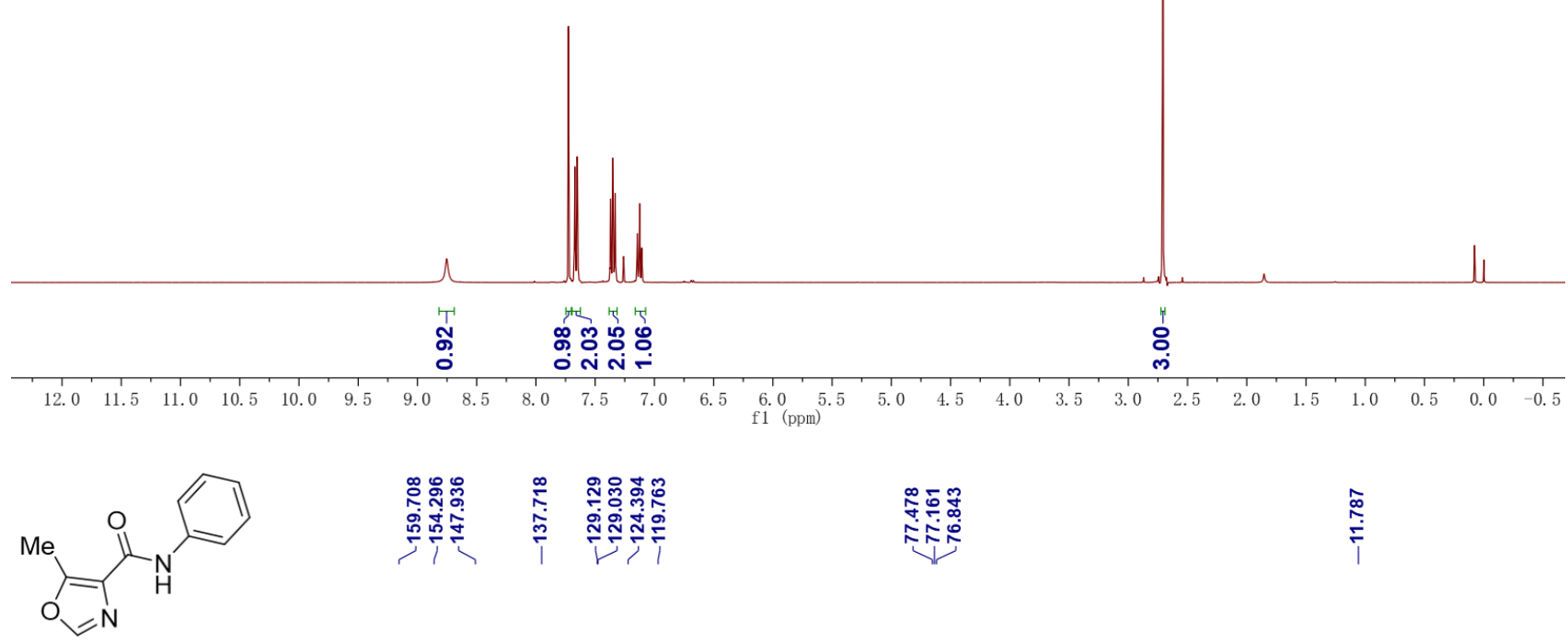

${ }^{13} \mathrm{C}$ NMR $\left(100 \mathrm{MHz}, \mathrm{CDCl}_{3}\right)$

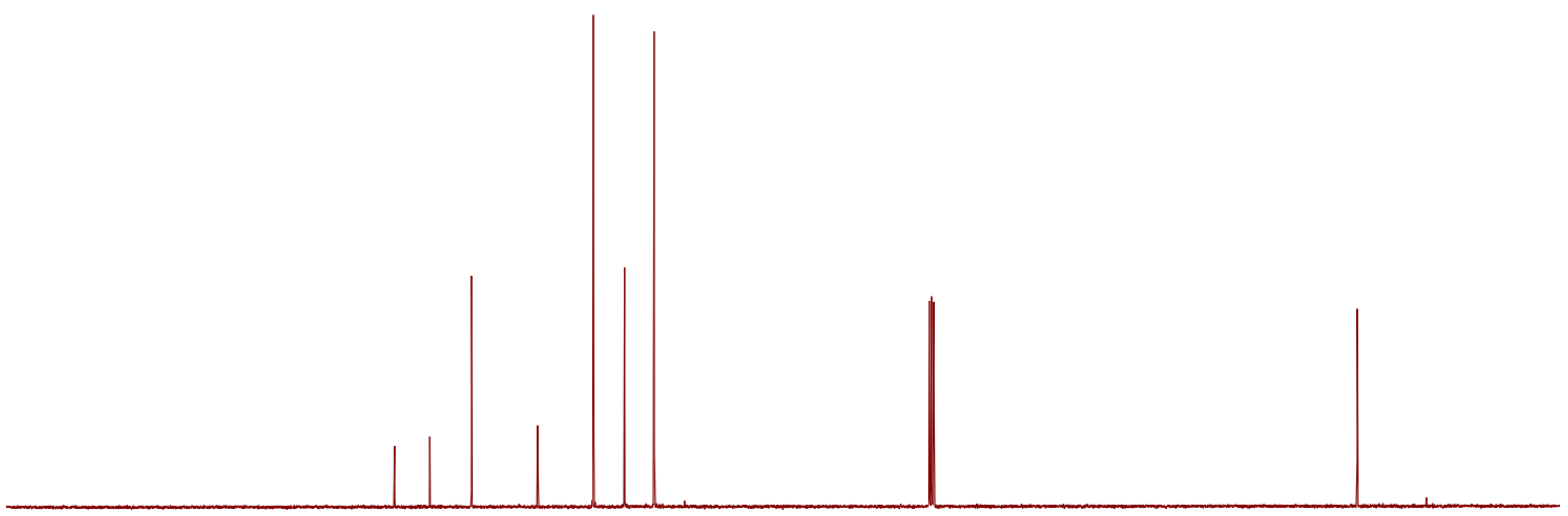

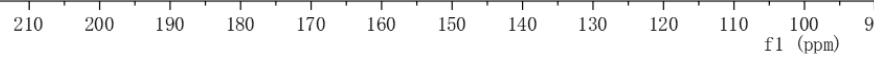

Figure S69. ${ }^{1} \mathrm{H}$ NMR and ${ }^{13} \mathrm{C}\left\{{ }^{1} \mathrm{H}\right\}$ NMR Spectra of 5-methyl-N-phenyloxazole-4-carboxamide (5w). 
<smiles>Cc1nc(C(=O)Nc2ccccc2)cs1</smiles>

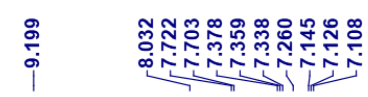

${ }^{1} \mathrm{H} \mathrm{NMR}\left(400 \mathrm{MHz}, \mathrm{CDCl}_{3}\right)$

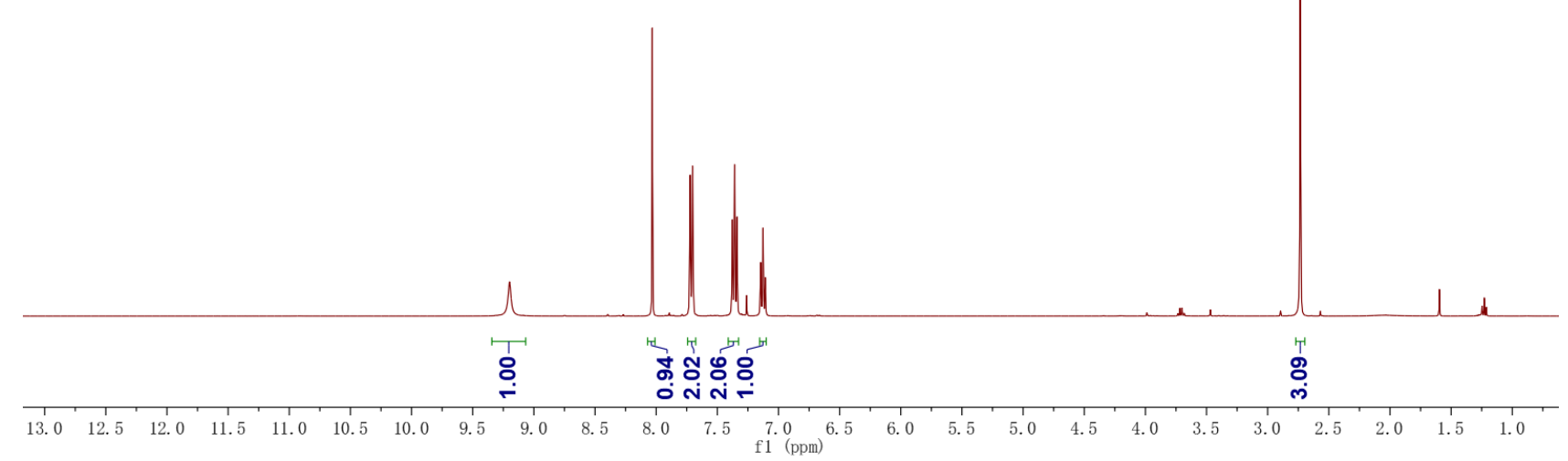<smiles>Cc1nc(C(=O)Nc2ccccc2)cs1</smiles>

${ }^{13} \mathrm{C}$ NMR $\left(100 \mathrm{MHz}, \mathrm{CDCl}_{3}\right)$

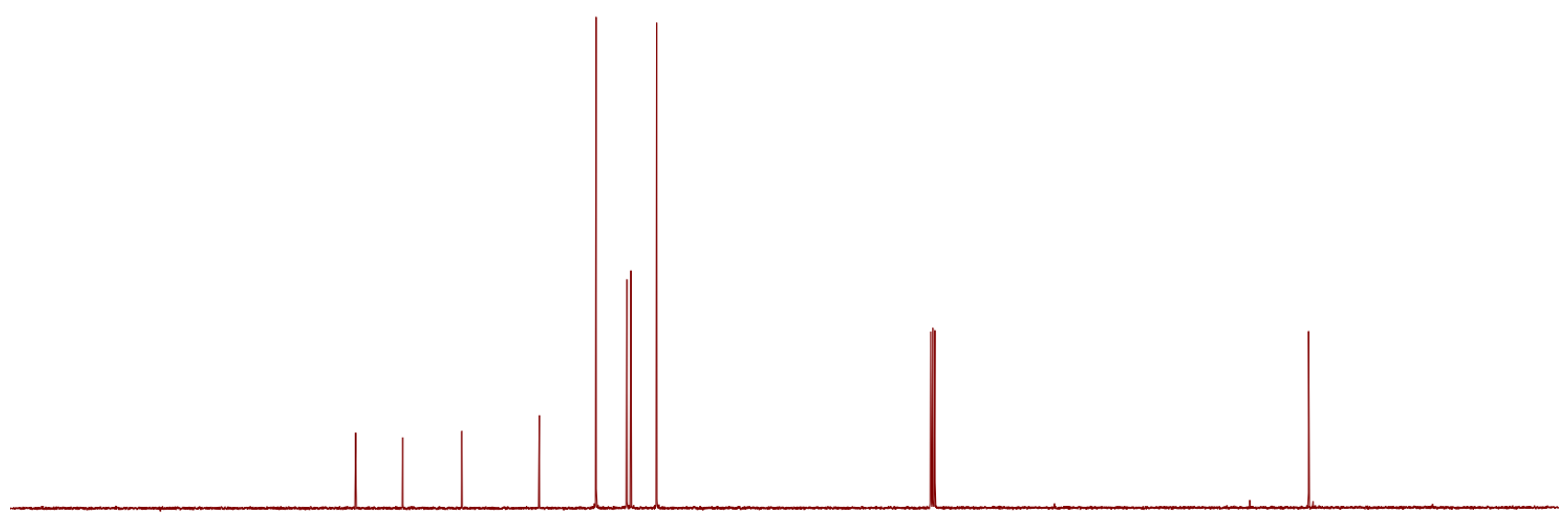

$\begin{array}{llllllllllll}210 & 200 & 190 & 180 & 170 & 160 & 150 & 140 & 130 & 120 & 110 & 100 \\ \mathrm{f} 1(\mathrm{ppm}) & 90\end{array}$

Figure S70. ${ }^{1} \mathrm{H}$ NMR and ${ }^{13} \mathrm{C}\left\{{ }^{1} \mathrm{H}\right\}$ NMR Spectra of 2-methyl- $N$-phenylthiazole-4-carboxamide $(\mathbf{5 x})$. 
<smiles>O=C(Nc1ccccc1)c1ccc2nc[nH]c2c1</smiles>

${ }^{1} \mathrm{H}$ NMR $\left(400 \mathrm{MHz}, \mathrm{DMSO}-d_{6}\right)$
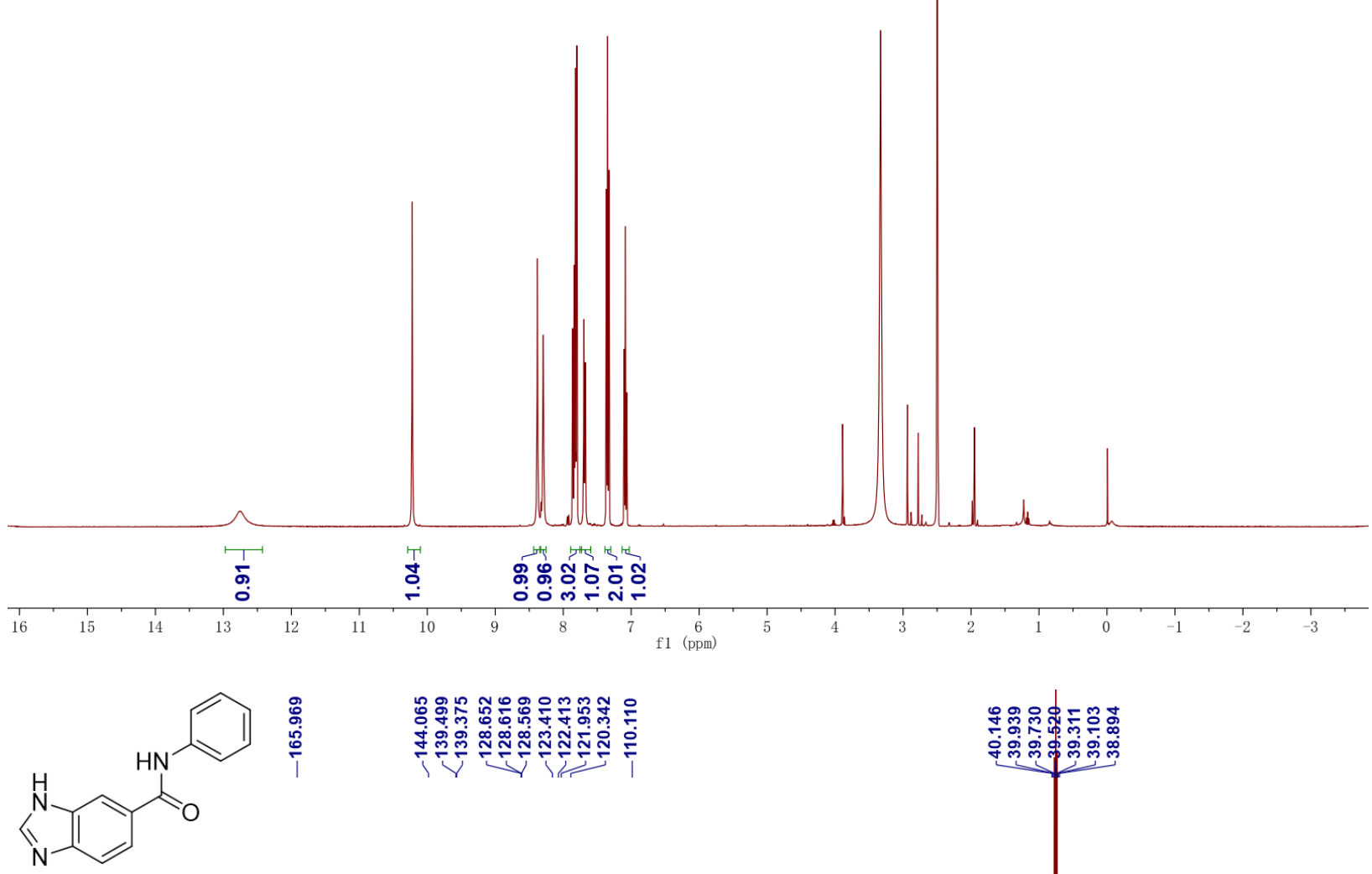

${ }^{13} \mathrm{C}$ NMR (400 MHz, DMSO- $\left.d_{6}\right)$

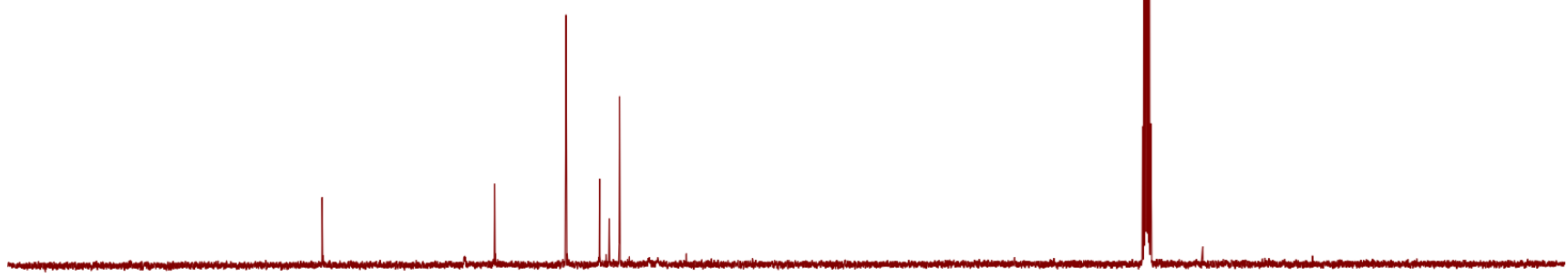

$\begin{array}{rrrrrrrrrrrr}210 & 200 & 190 & 180 & 170 & 160 & 150 & 140 & 130 & 120 & 110 & 100 \\ \mathrm{f} 1 & (\mathrm{ppm})\end{array}$

Figure S71. ${ }^{1} \mathrm{H}$ NMR and ${ }^{13} \mathrm{C}\left\{{ }^{1} \mathrm{H}\right\}$ NMR Spectra of $N$-phenyl-1H-benzo[d]imidazole-6-carboxamide (5y). 
<smiles>O=C(Cc1ccccc1)Nc1ccccc1</smiles>

${ }^{1} \mathrm{H} \mathrm{NMR}\left(400 \mathrm{MHz}, \mathrm{CDCl}_{3}\right)$
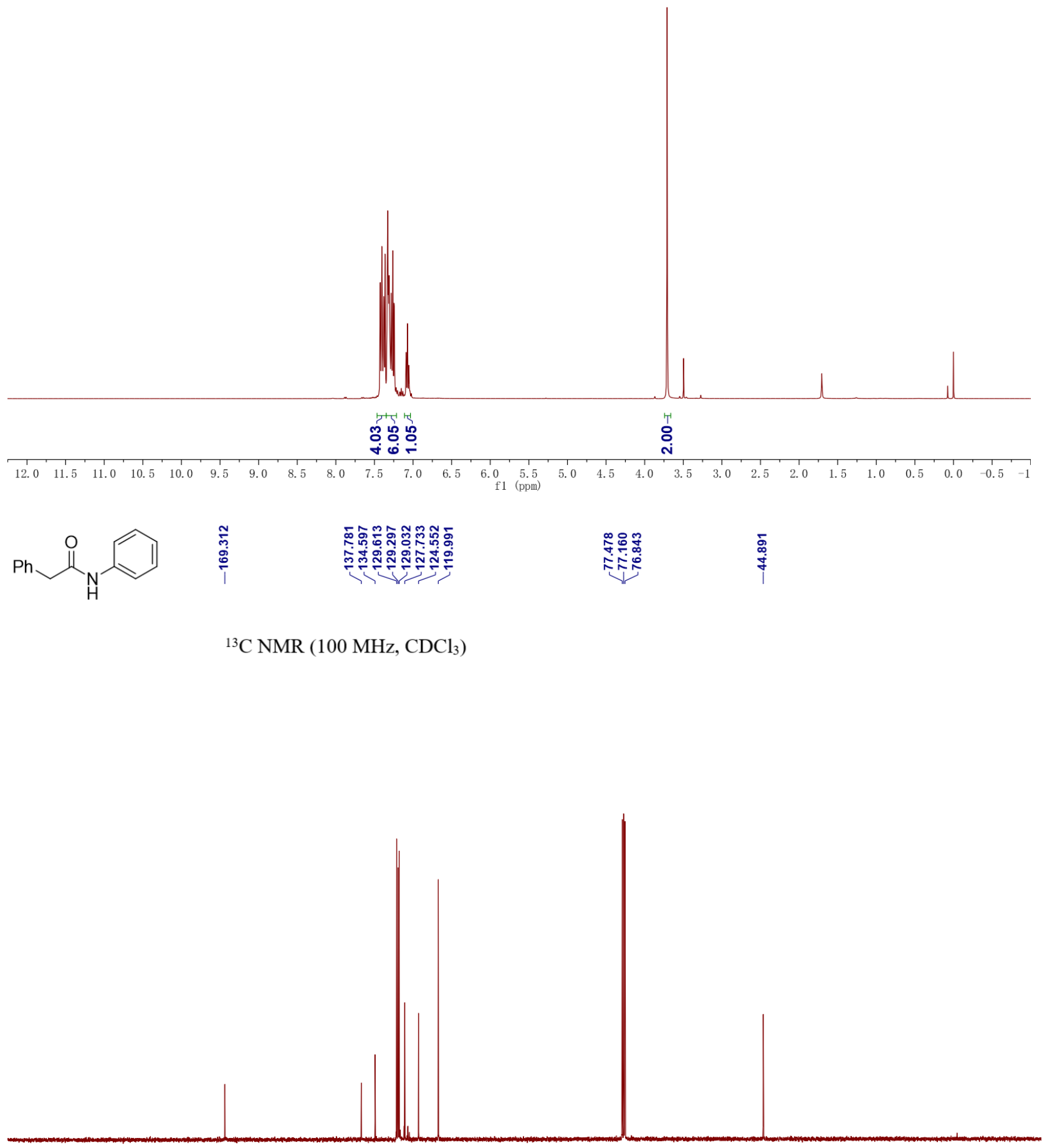

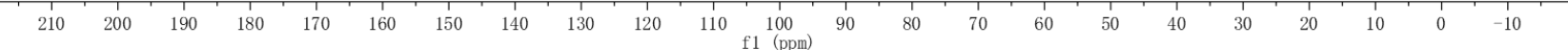

Figure S72. ${ }^{1} \mathrm{H}$ NMR and ${ }^{13} \mathrm{C}\left\{{ }^{1} \mathrm{H}\right\}$ NMR Spectra of N,2-diphenylacetamide (6a). 
<smiles>O=C(Nc1ccccc1)C1CCOCC1</smiles>

\section{సิำ

${ }^{1} \mathrm{H}$ NMR $\left(400 \mathrm{MHz}, \mathrm{CDCl}_{3}\right)$

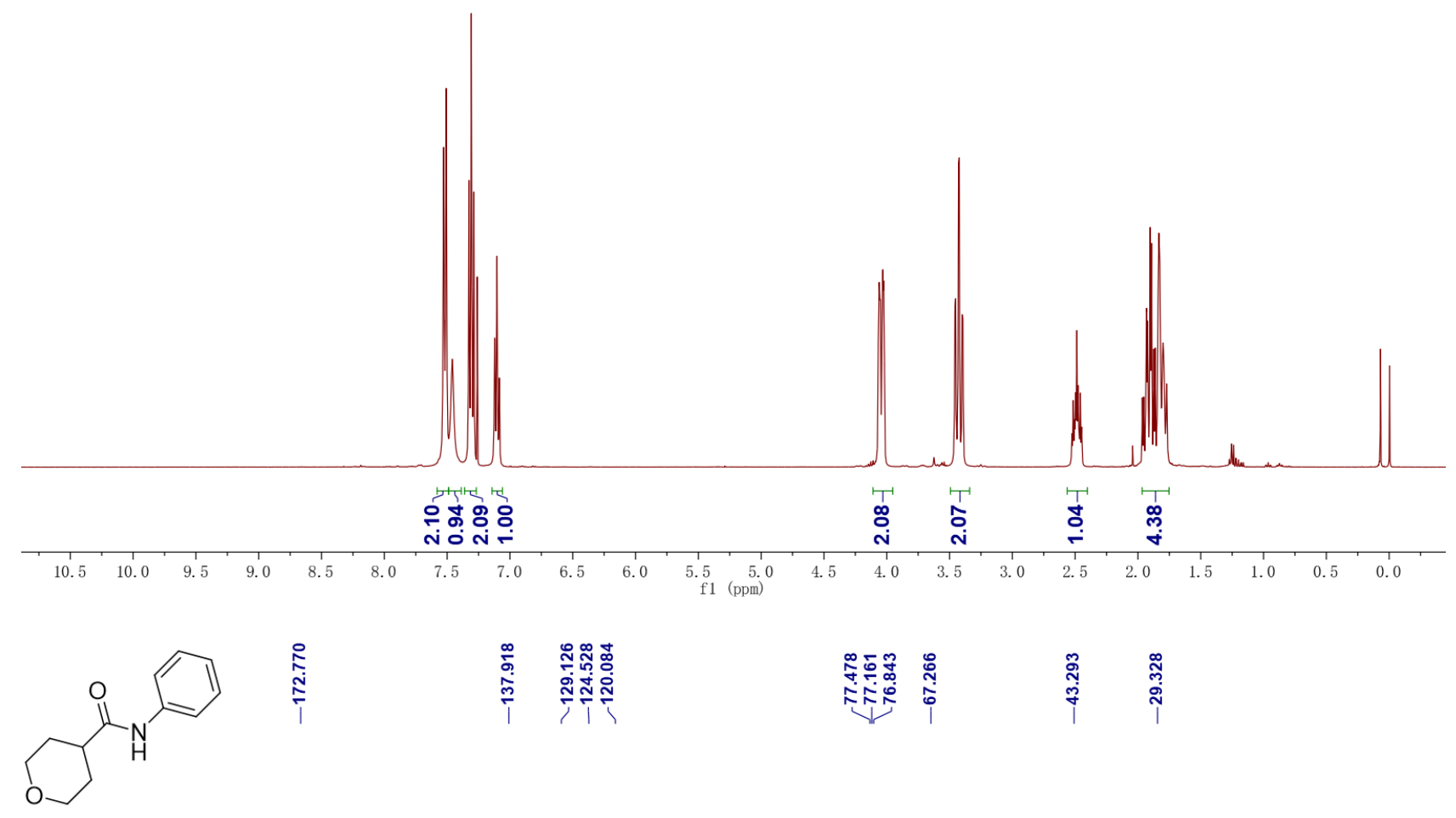

${ }^{13} \mathrm{C}$ NMR $\left(100 \mathrm{MHz}, \mathrm{CDCl}_{3}\right)$

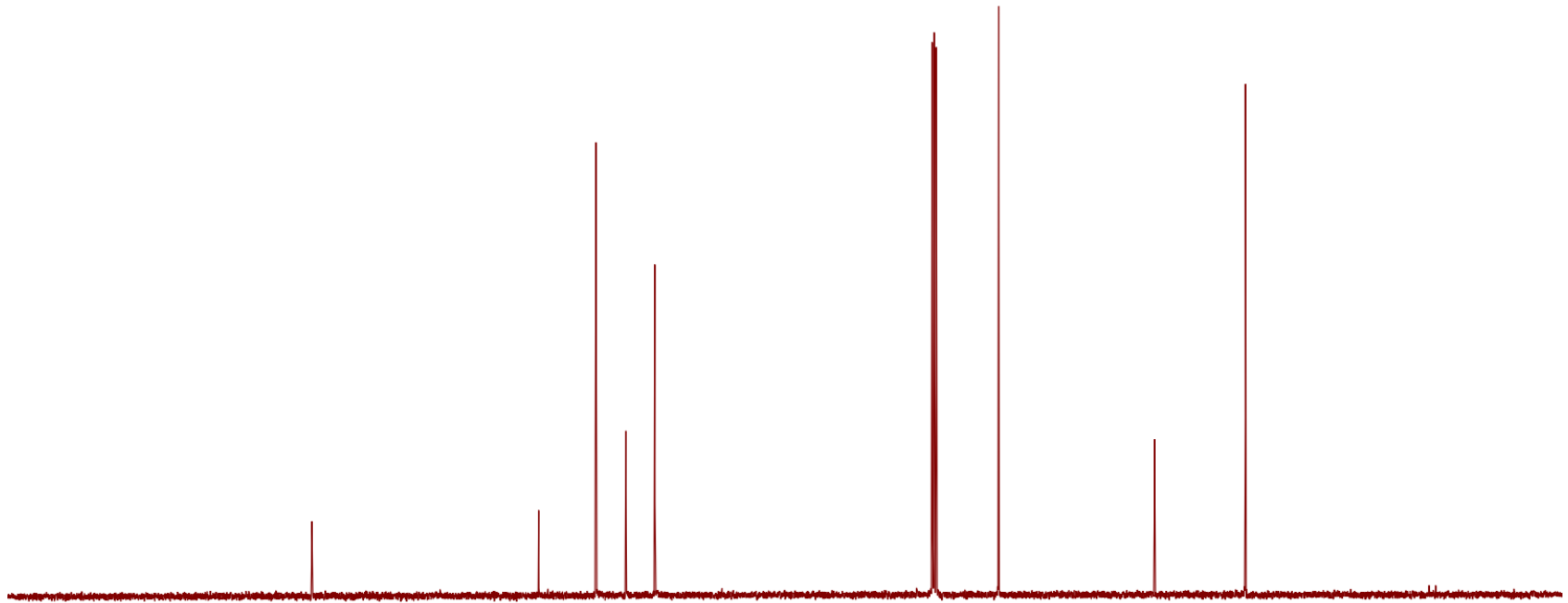

$\begin{array}{llllllllllll}210 & 200 & 190 & 180 & 170 & 160 & 150 & 140 & 130 & 120 & 110 & 100\end{array}$

Figure S73. ${ }^{1} \mathrm{H}$ NMR and ${ }^{13} \mathrm{C}\left\{{ }^{1} \mathrm{H}\right\}$ NMR Spectra of tetrahydro-pyran-4-carboxylic acid anilide (6b) 

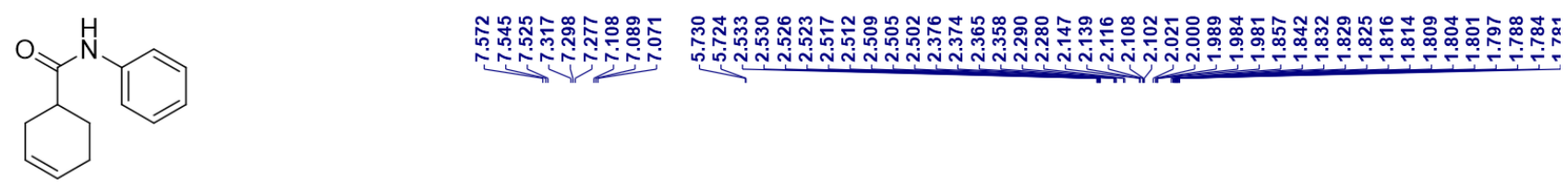

${ }^{1} \mathrm{H} \mathrm{NMR}\left(400 \mathrm{MHz}, \mathrm{CDCl}_{3}\right)$

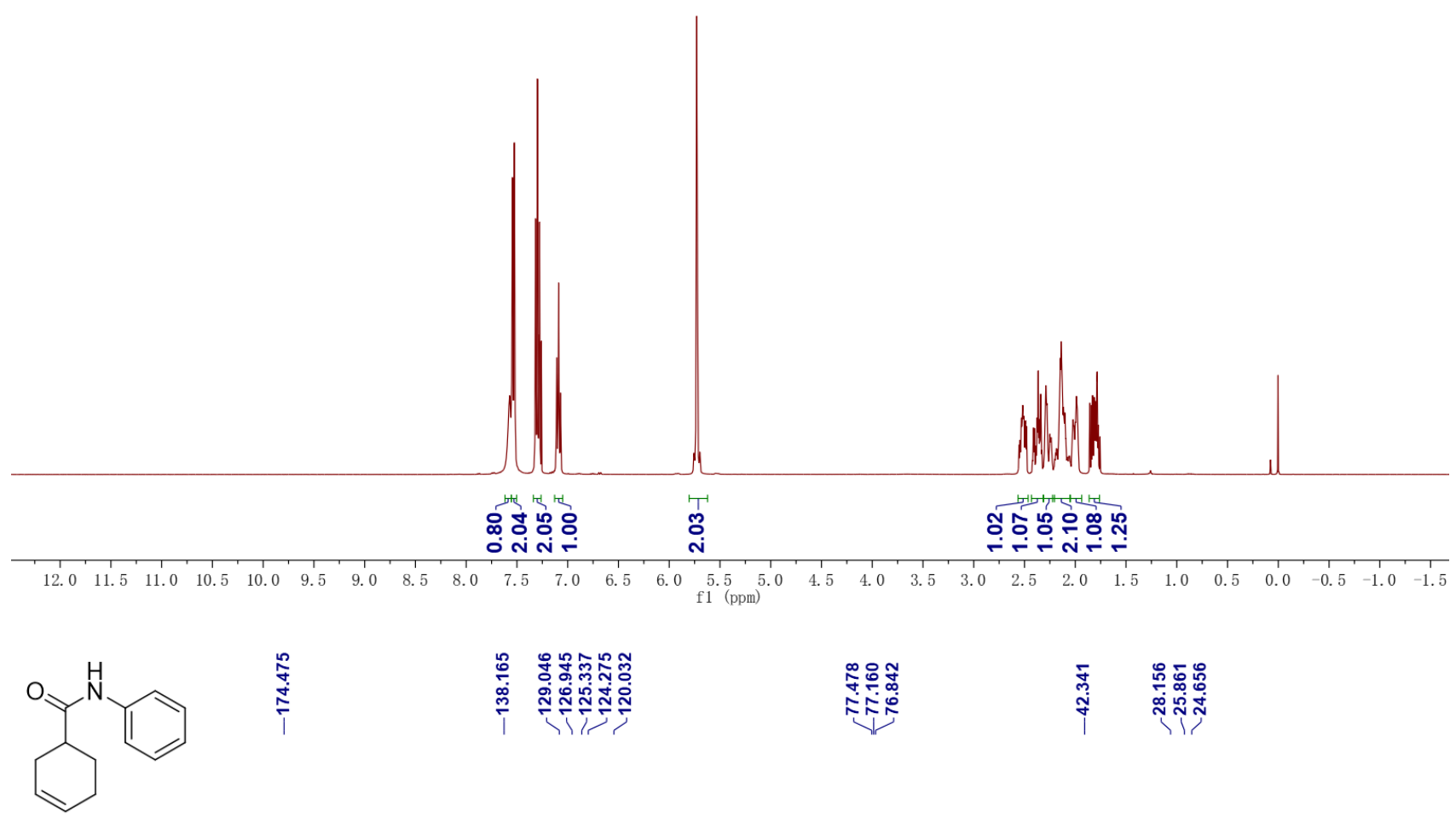

${ }^{13} \mathrm{C} \mathrm{NMR}\left(100 \mathrm{MHz}, \mathrm{CDCl}_{3}\right)$

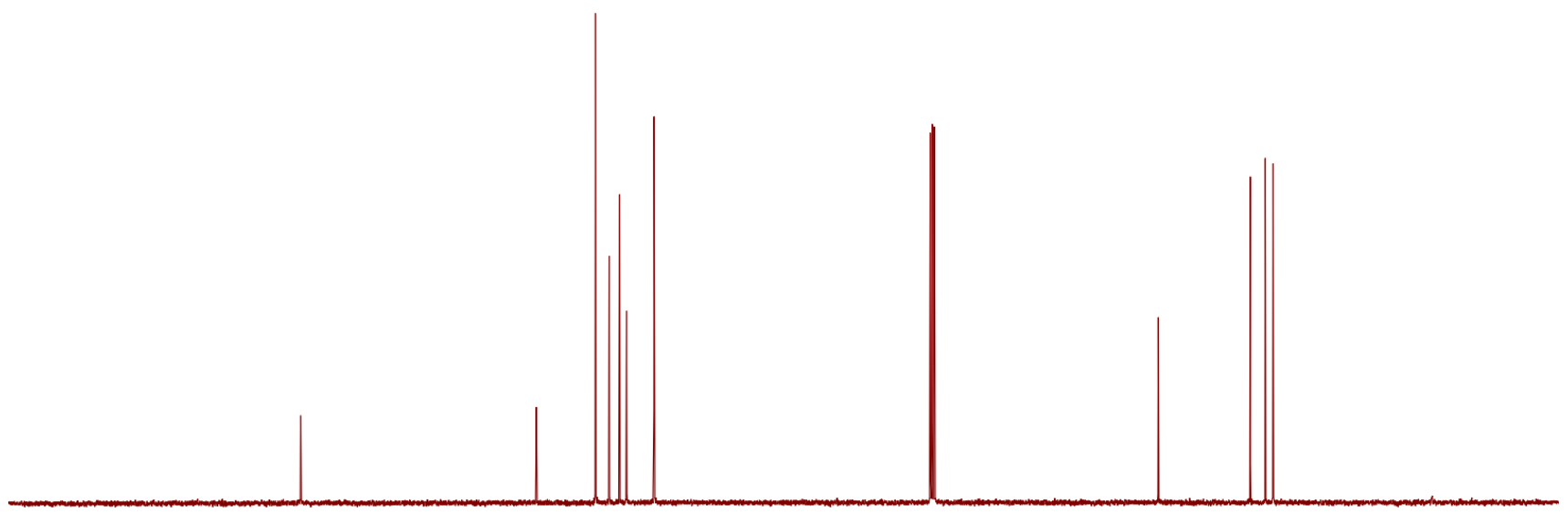

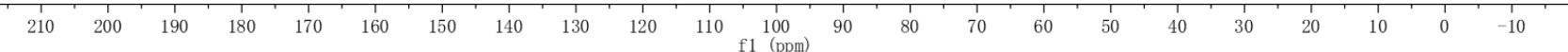

Figure S74. ${ }^{1} \mathrm{H}$ NMR and ${ }^{13} \mathrm{C}\left\{{ }^{1} \mathrm{H}\right\}$ NMR Spectra of $N$-phenylcyclohex-3-ene-1-carboxamide anilide $(\mathbf{6 c})$. 


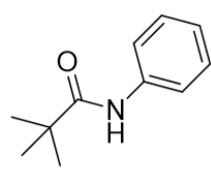

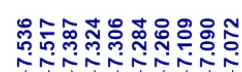

${ }^{1} \mathrm{H}$ NMR (400 MHz, $\left.\mathrm{CDCl}_{3}\right)$

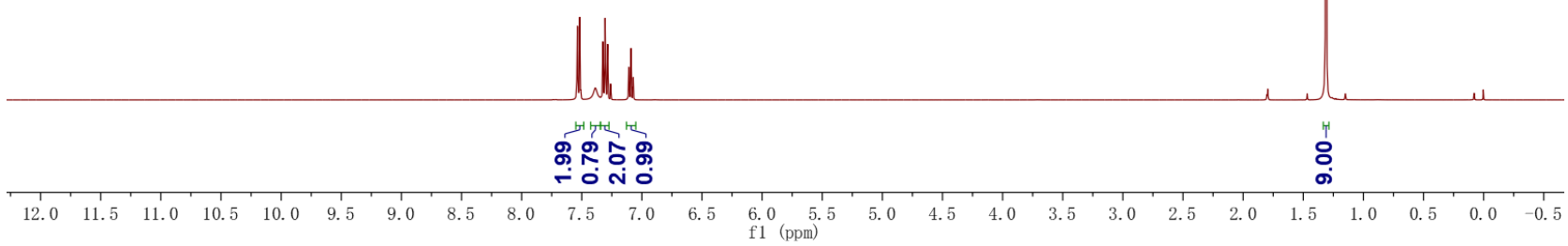

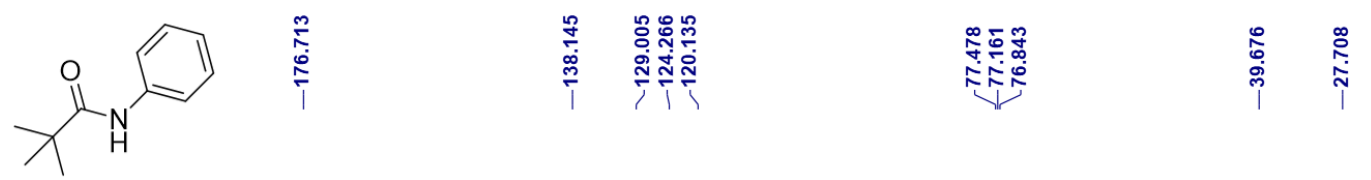

${ }^{13} \mathrm{C}$ NMR $\left(100 \mathrm{MHz}, \mathrm{CDCl}_{3}\right)$

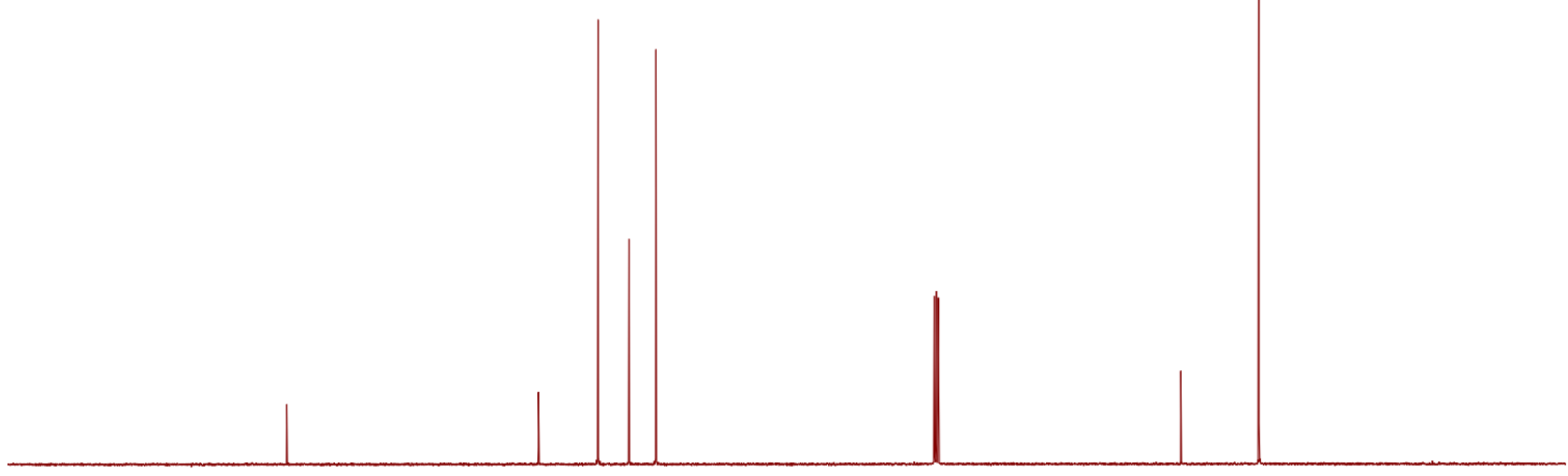

$\begin{array}{llllllllllllllllllllllll}1 & 1 \\ 210 & 200 & 190 & 180 & 170 & 160 & 150 & 140 & 130 & 120 & 110 & 100 & 90 & 80 & 70 & 60 & 50 & 40 & 30 & 20 & 10 & 0 & -10 & 1\end{array}$

Figure S75. ${ }^{1} \mathrm{H}$ NMR and ${ }^{13} \mathrm{C}\left\{{ }^{1} \mathrm{H}\right\}$ NMR Spectra of $N$-pivaloylaniline (6d). 


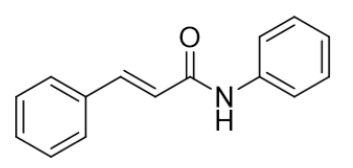

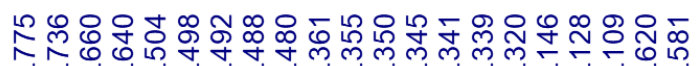

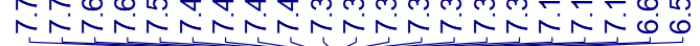

${ }^{1} \mathrm{H} \mathrm{NMR}\left(400 \mathrm{MHz}, \mathrm{CDCl}_{3}\right)$

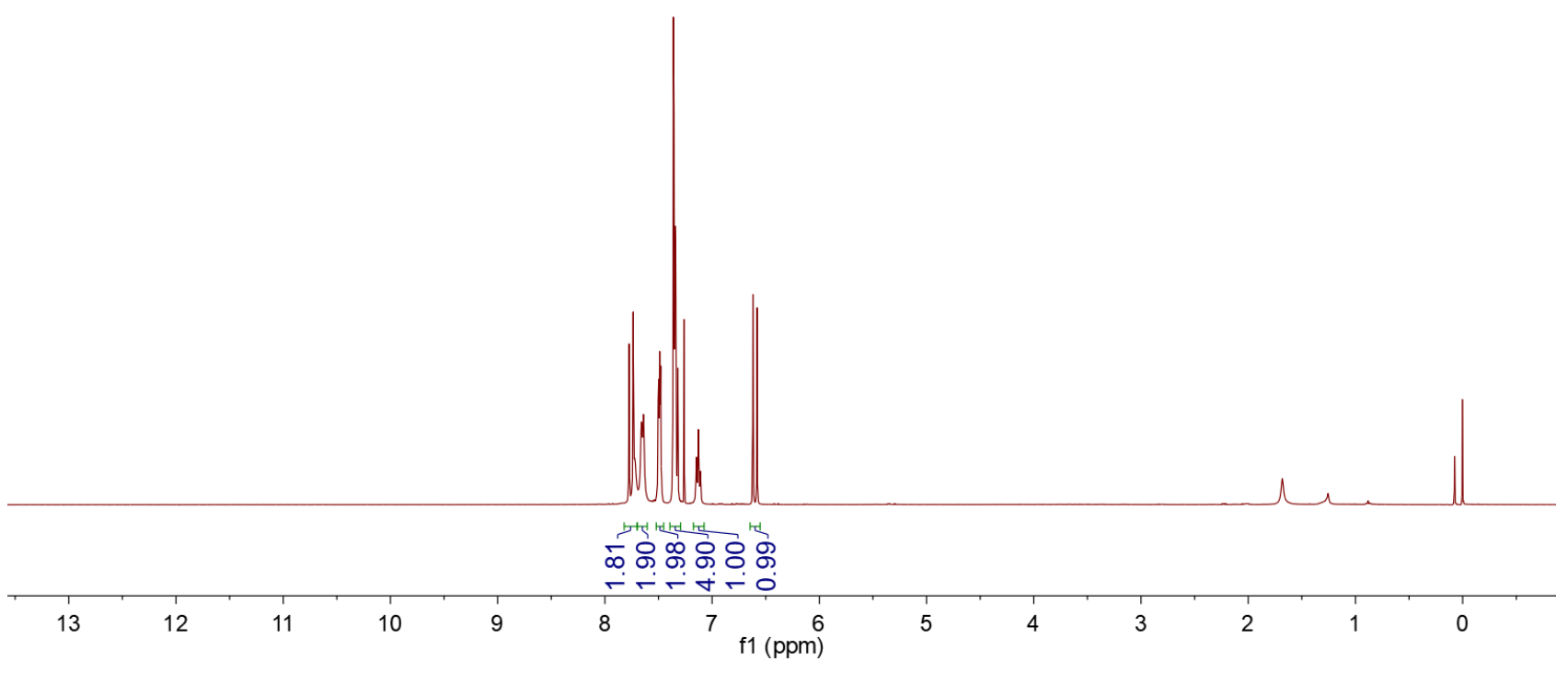

14

${ }^{13} \mathrm{C} \mathrm{NMR}\left(100 \mathrm{MHz}, \mathrm{CDCl}_{3}\right)$

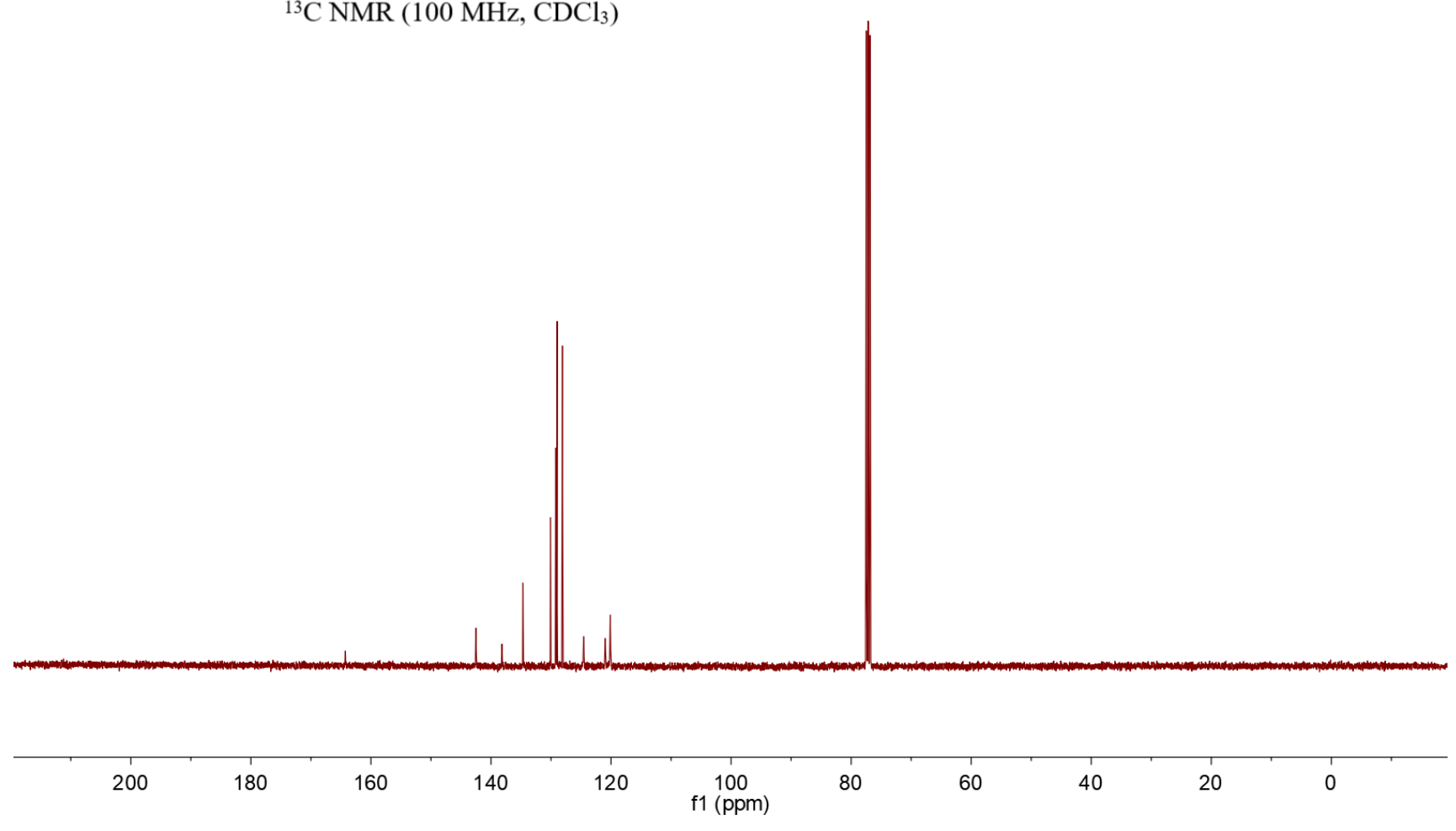

Figure S76. ${ }^{1} \mathrm{H}$ NMR and ${ }^{13} \mathrm{C}\left\{{ }^{1} \mathrm{H}\right\}$ NMR Spectra of N-phenylcinnamamide (6e). 

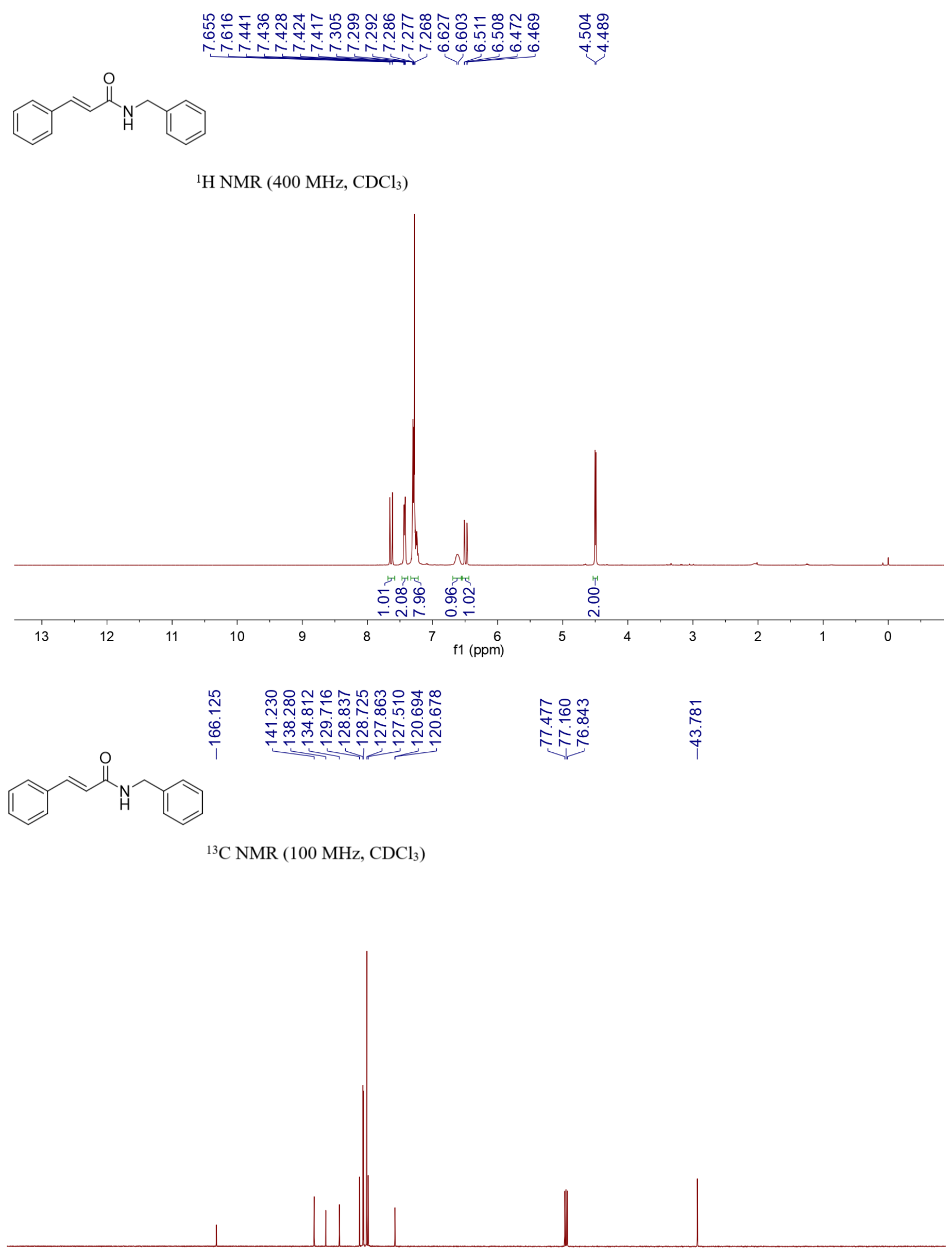

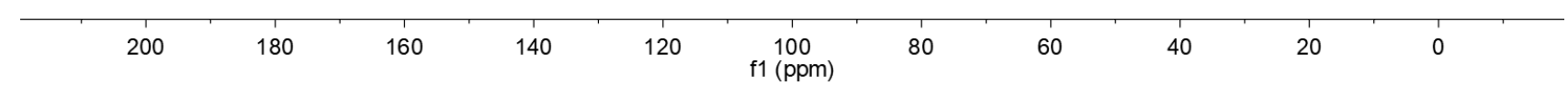

Figure S77. ${ }^{1} \mathrm{H}$ NMR and ${ }^{13} \mathrm{C}\left\{{ }^{1} \mathrm{H}\right\}$ NMR Spectra of N-benzylcinnamamide (6f). 
$\mathrm{N}_{\mathrm{H}}^{-\mathrm{C}_{6} \mathrm{H}_{13}}$

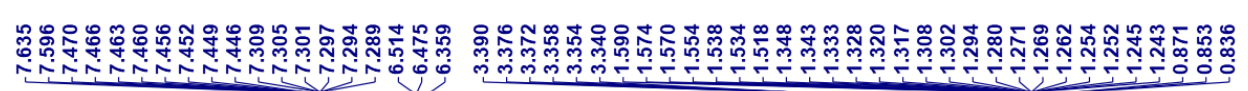

${ }^{1} \mathrm{H}$ NMR $\left(400 \mathrm{MHz}, \mathrm{CDCl}_{3}\right)$

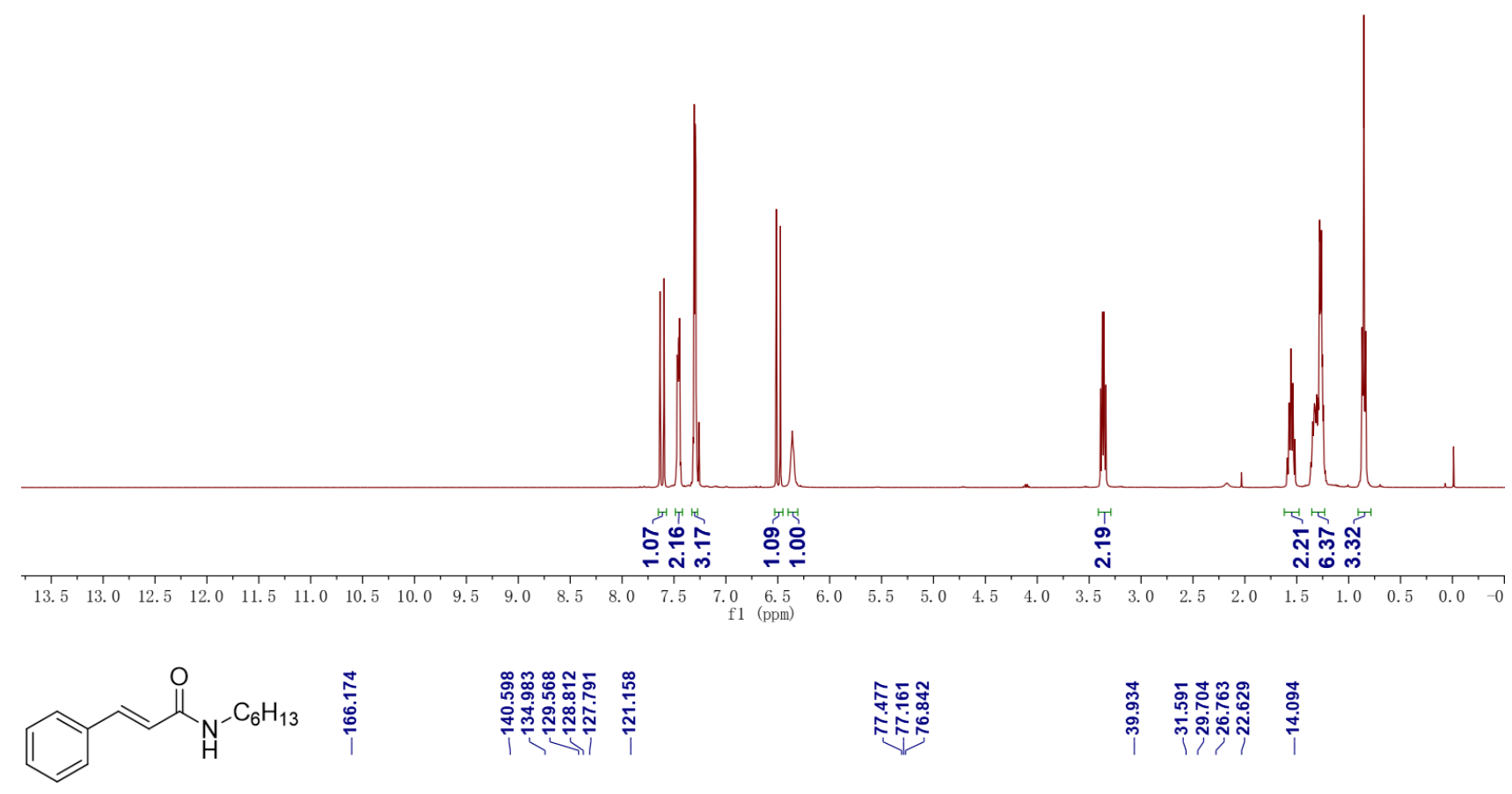

${ }^{13} \mathrm{C} \mathrm{NMR}\left(100 \mathrm{MHz}, \mathrm{CDCl}_{3}\right)$

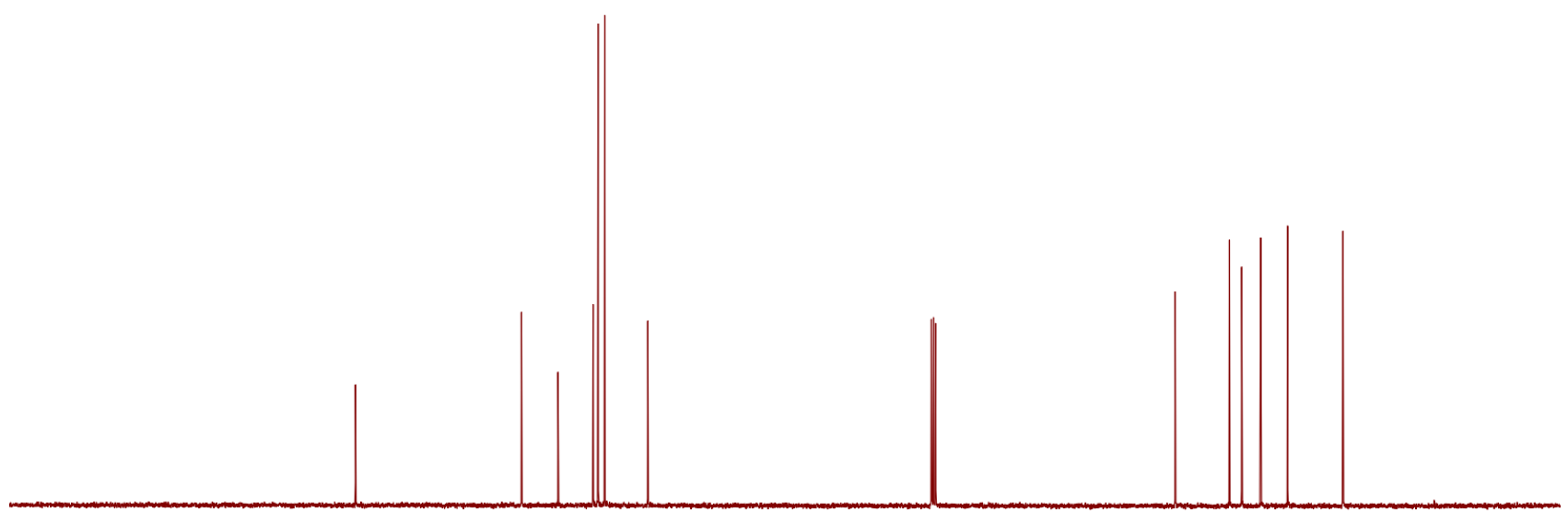

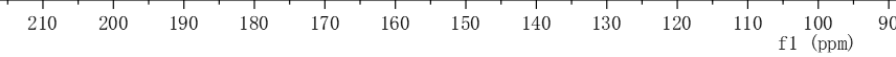

Figure S78. ${ }^{1} \mathrm{H}$ NMR and ${ }^{13} \mathrm{C}\left\{{ }^{1} \mathrm{H}\right\}$ NMR Spectra of N-hexylcinnamamide (6g). 


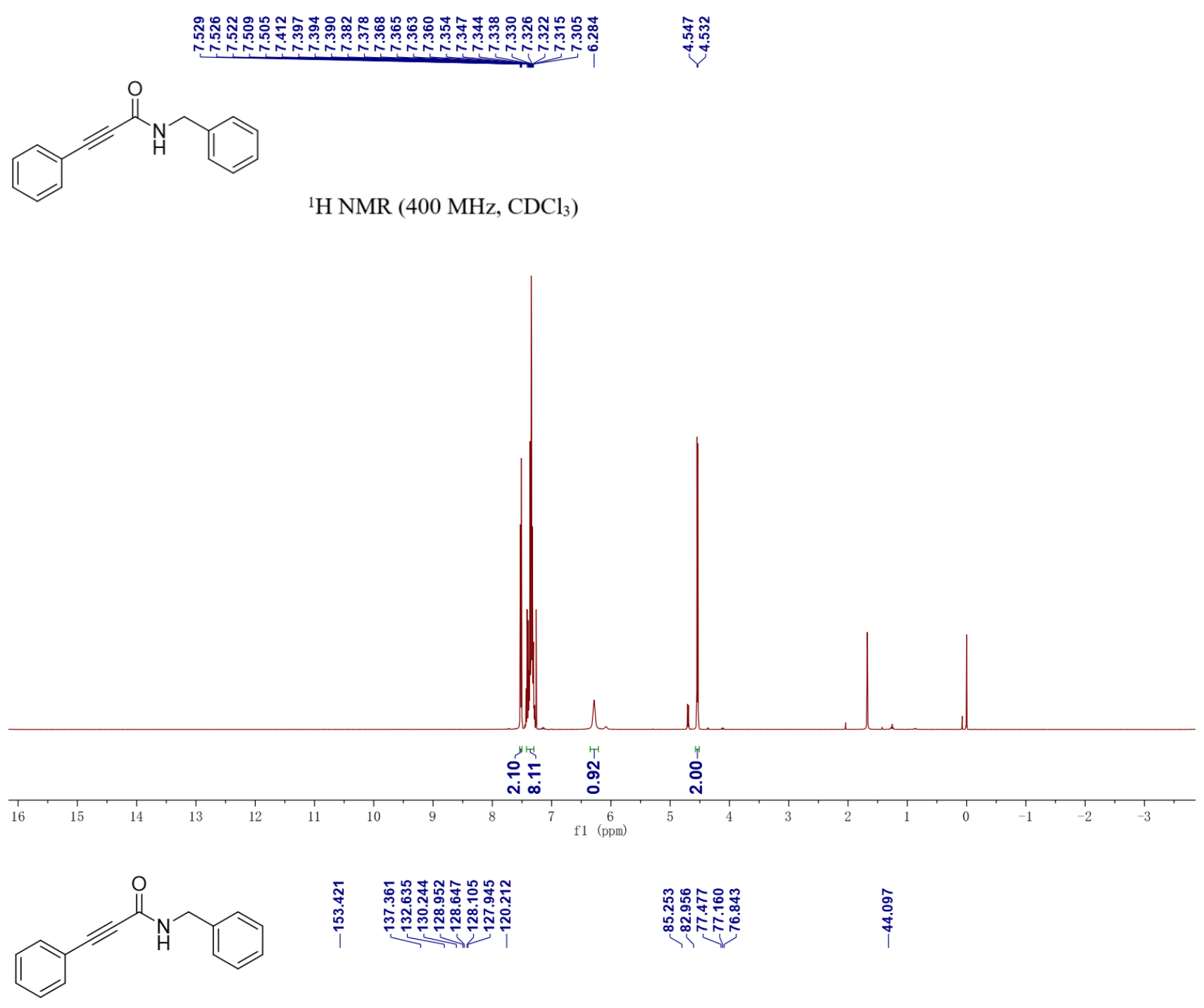

${ }^{13} \mathrm{C} \mathrm{NMR}\left(100 \mathrm{MHz}, \mathrm{CDCl}_{3}\right)$

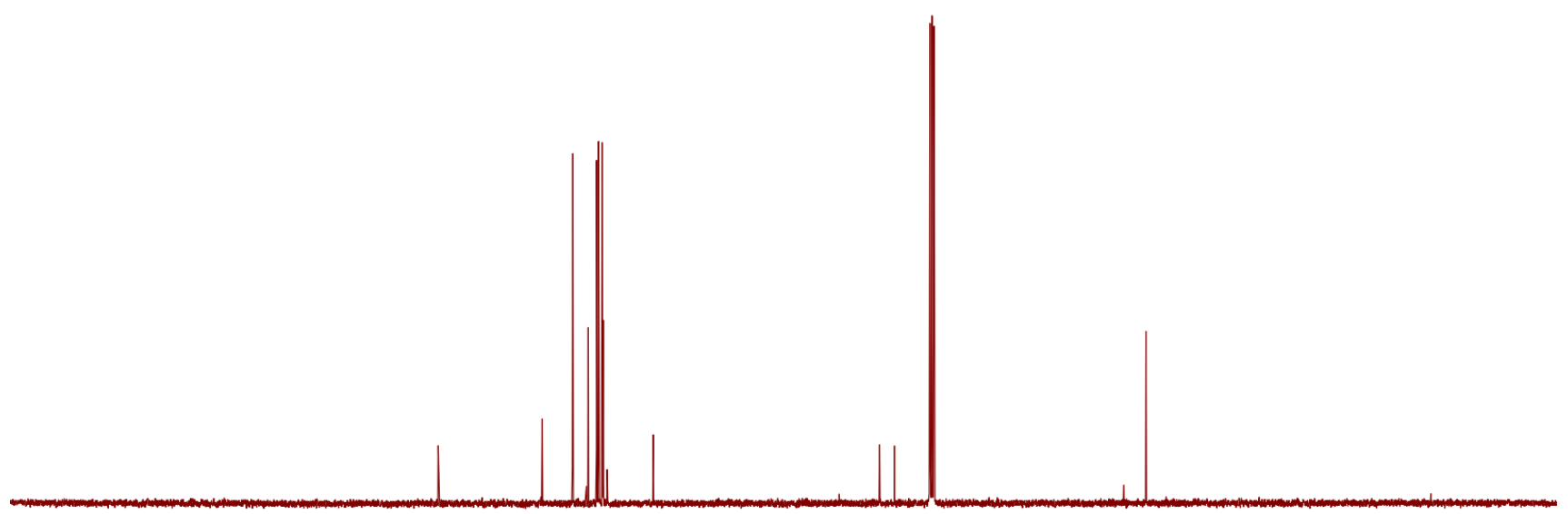

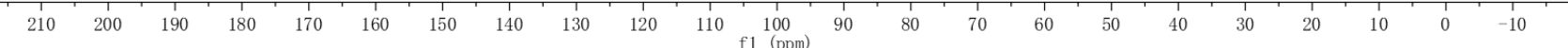

Figure S79. ${ }^{1} \mathrm{H}$ NMR and ${ }^{13} \mathrm{C}\left\{{ }^{1} \mathrm{H}\right\}$ NMR Spectra of N-benzyl-3-phenylpropiolamide (6h). 


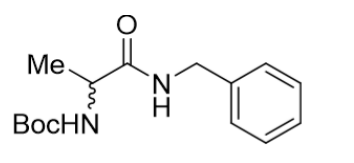

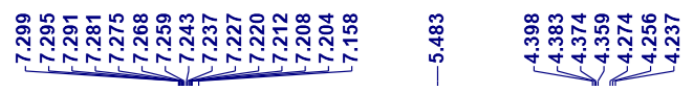

${ }^{1} \mathrm{H} \mathrm{NMR}\left(400 \mathrm{MHz}, \mathrm{CDCl}_{3}\right)$

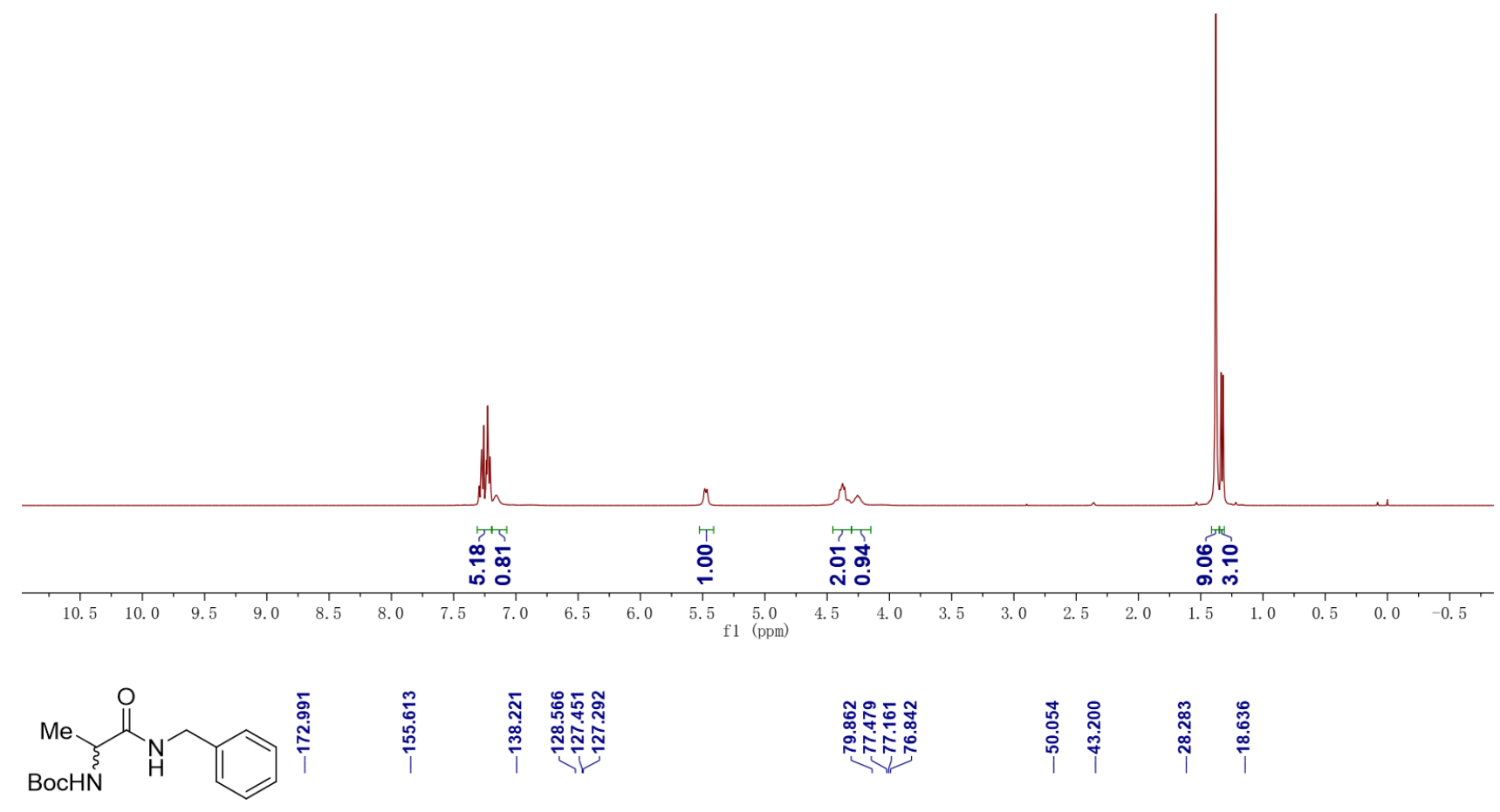

${ }^{13} \mathrm{C} \mathrm{NMR}\left(100 \mathrm{MHz}, \mathrm{CDCl}_{3}\right)$

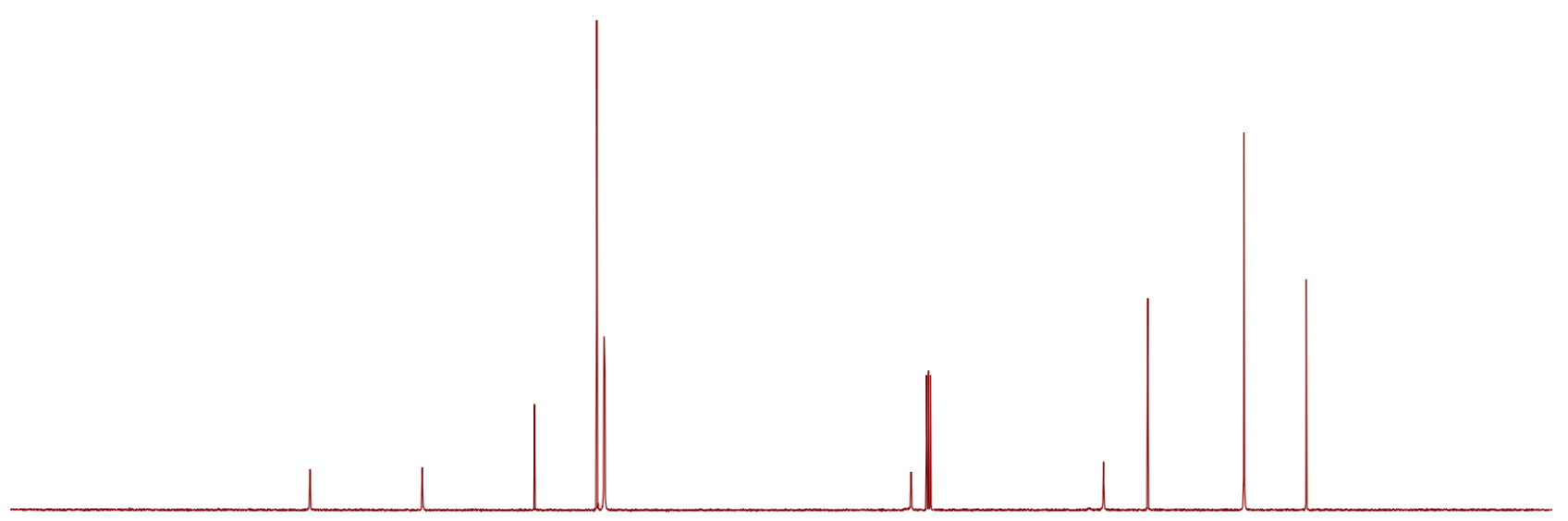

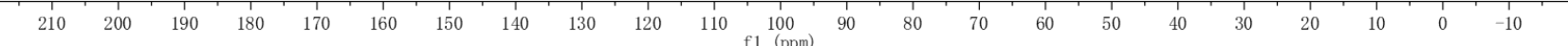

Figure S80. ${ }^{1} \mathrm{H}$ NMR and ${ }^{13} \mathrm{C}\left\{{ }^{1} \mathrm{H}\right\}$ NMR Spectra of tert-butyl (1-(benzylamino)-1-oxopropan-2-yl)carbamate (6i). 


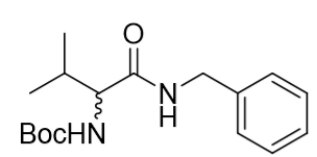

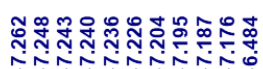

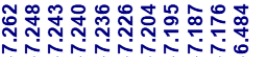

证。
商

萬

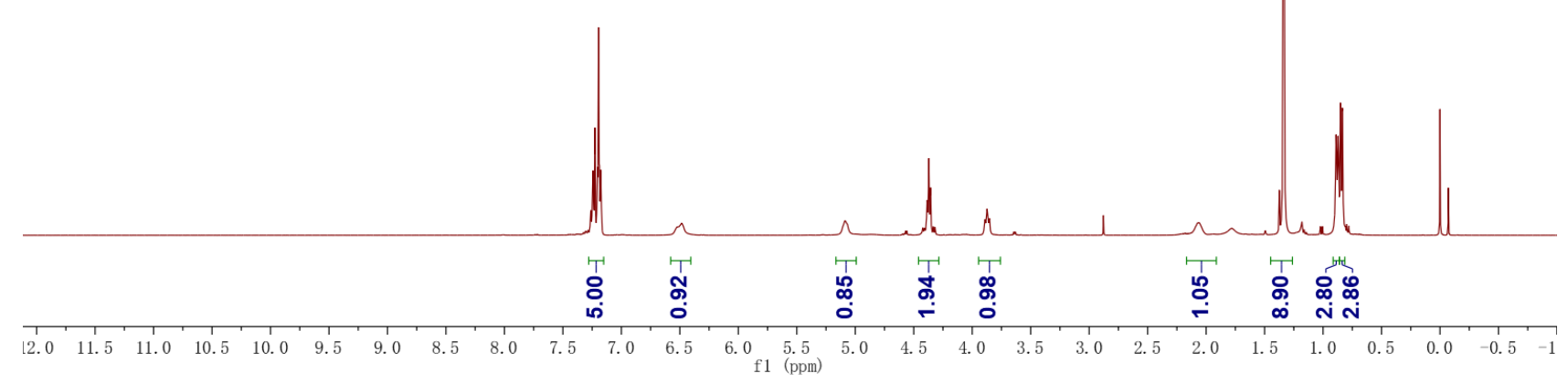

BochN

${ }^{13} \mathrm{C}$ NMR $\left(100 \mathrm{MHz}, \mathrm{CDCl}_{3}\right)$

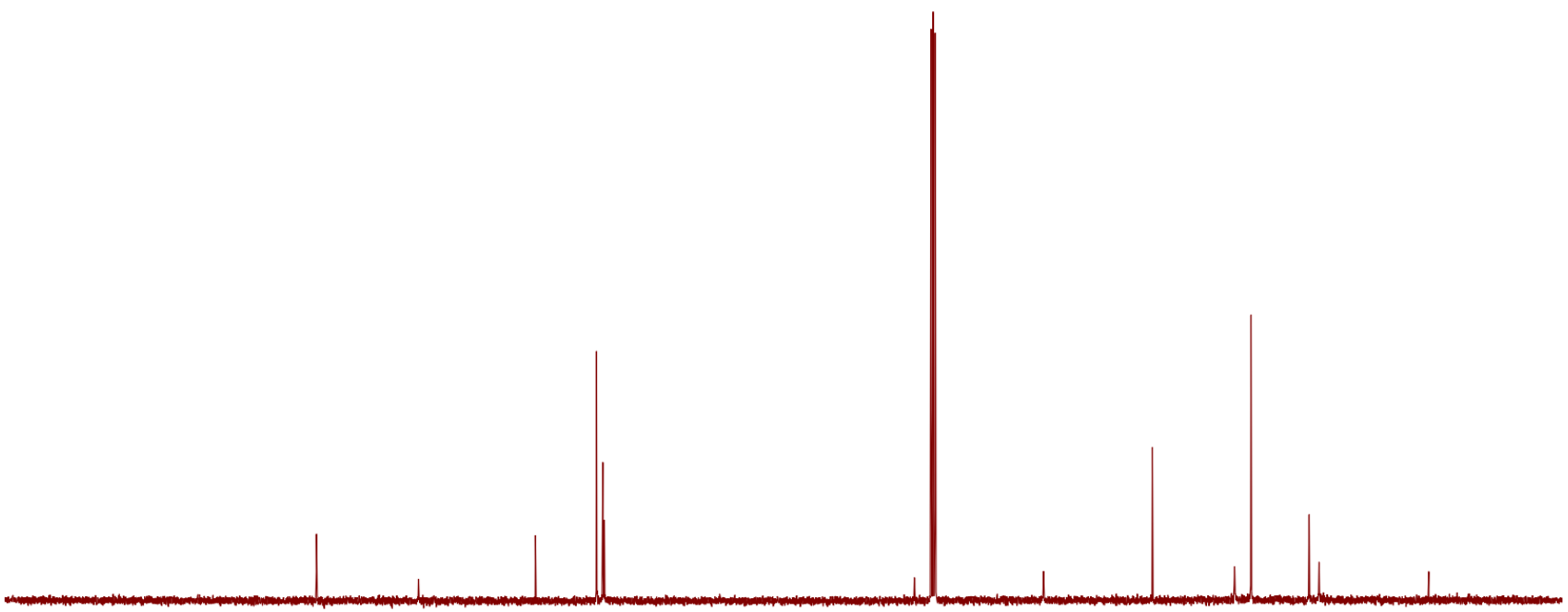

Figure S81. ${ }^{1} \mathrm{H}$ NMR and ${ }^{13} \mathrm{C}\left\{{ }^{1} \mathrm{H}\right\}$ NMR Spectra of tert-butyl (1-(benzylamino)-3-methyl-1-oxobutan-2yl)carbamate (6j). 
(N)

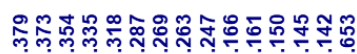

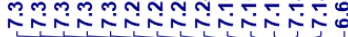

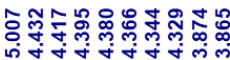

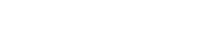

${ }^{1} \mathrm{H} \mathrm{NMR}\left(400 \mathrm{MHz}, \mathrm{CDCl}_{3}\right)$
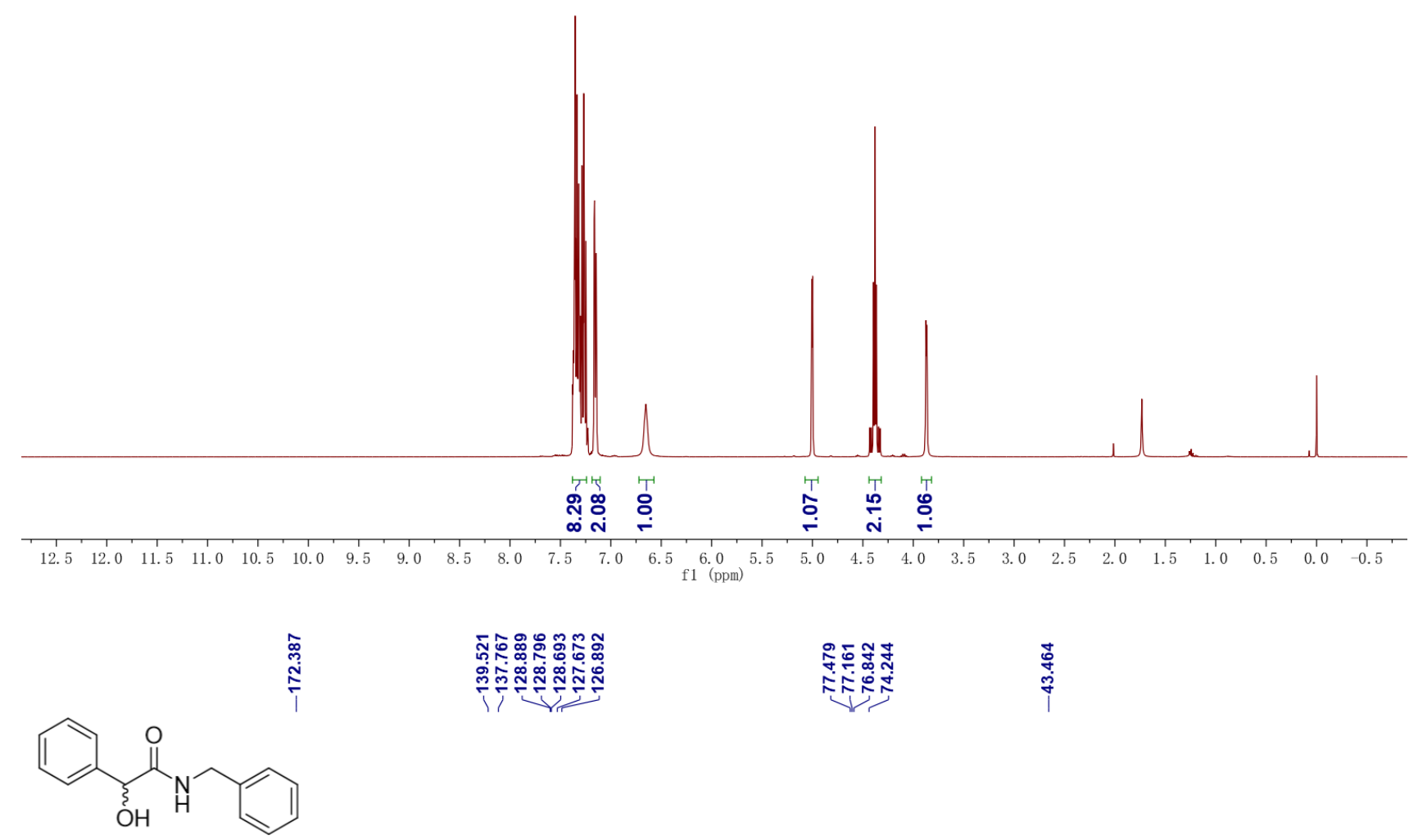

${ }^{13} \mathrm{C} \mathrm{NMR}\left(100 \mathrm{MHz}, \mathrm{CDCl}_{3}\right)$

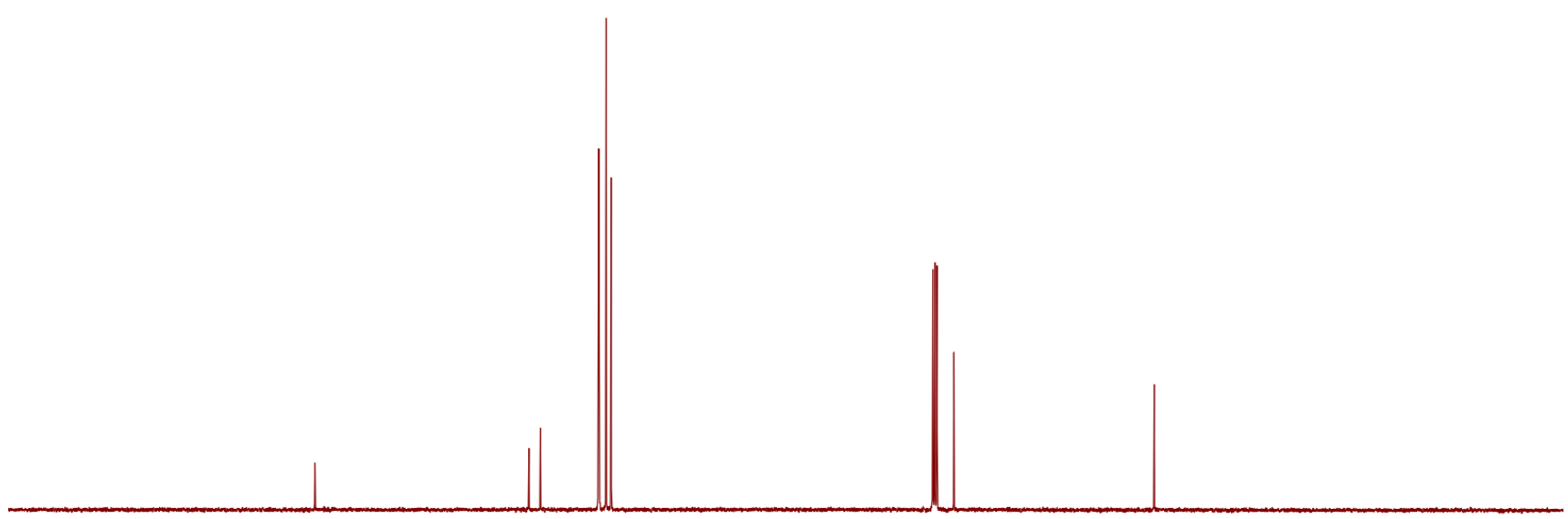

$\begin{array}{lllllll}210 & 200 & 190 & 180 & 170 & 160 & 150\end{array}$

Figure S82. ${ }^{1} \mathrm{H}$ NMR and ${ }^{13} \mathrm{C}\left\{{ }^{1} \mathrm{H}\right\}$ NMR Spectra of N-Benzylmandelamide (6k). 
$\mathrm{Me} \mathrm{H}^{7}=\mathrm{H}_{7}$

${ }^{1} \mathrm{H} \mathrm{NMR}\left(400 \mathrm{MHz}, \mathrm{CDCl}_{3}\right)$

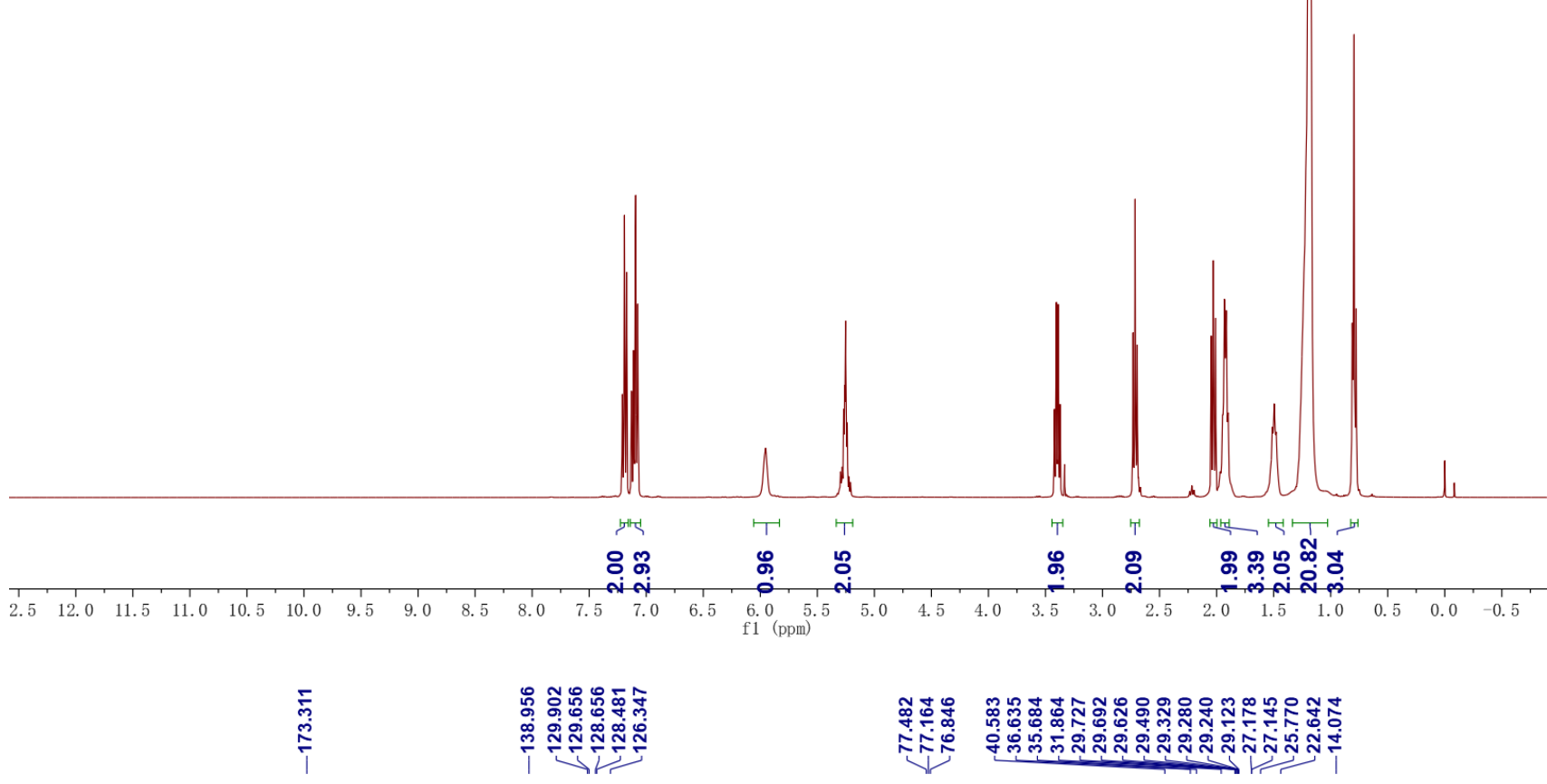<smiles>CCCCCCCC(=O)NCCc1ccccc1</smiles>

${ }^{13} \mathrm{C}$ NMR $\left(100 \mathrm{MHz}, \mathrm{CDCl}_{3}\right)$

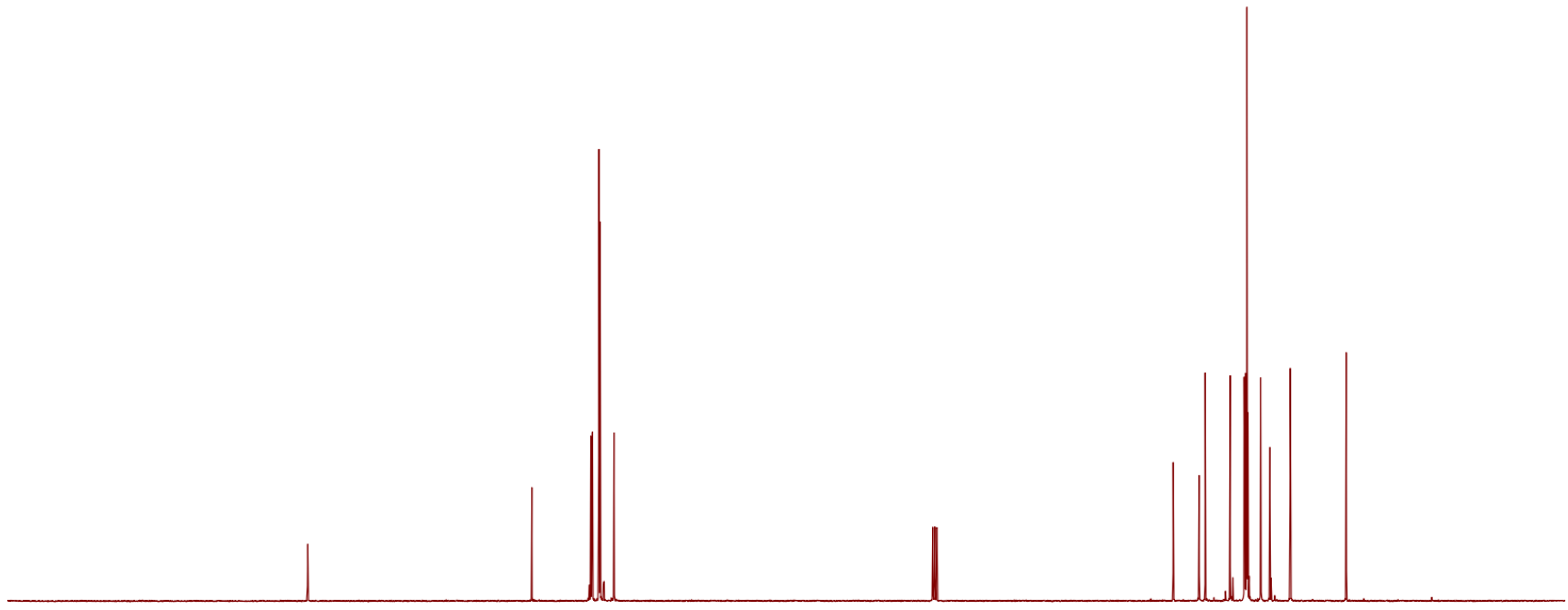

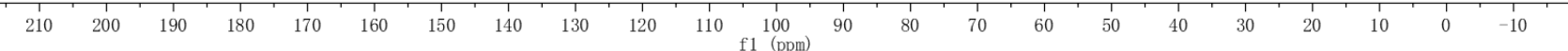

Figure S83. ${ }^{1} \mathrm{H}$ NMR and ${ }^{13} \mathrm{C}\left\{{ }^{1} \mathrm{H}\right\}$ NMR Spectra of $N$-phenethyloleamide (7a). 


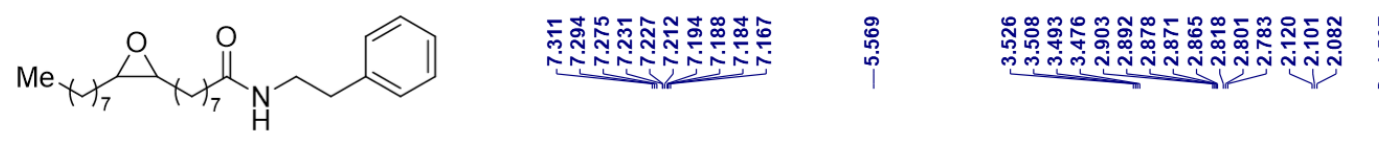

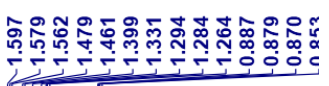

${ }^{1} \mathrm{H}$ NMR (400 MHz, $\mathrm{CDCl}_{3}$ )

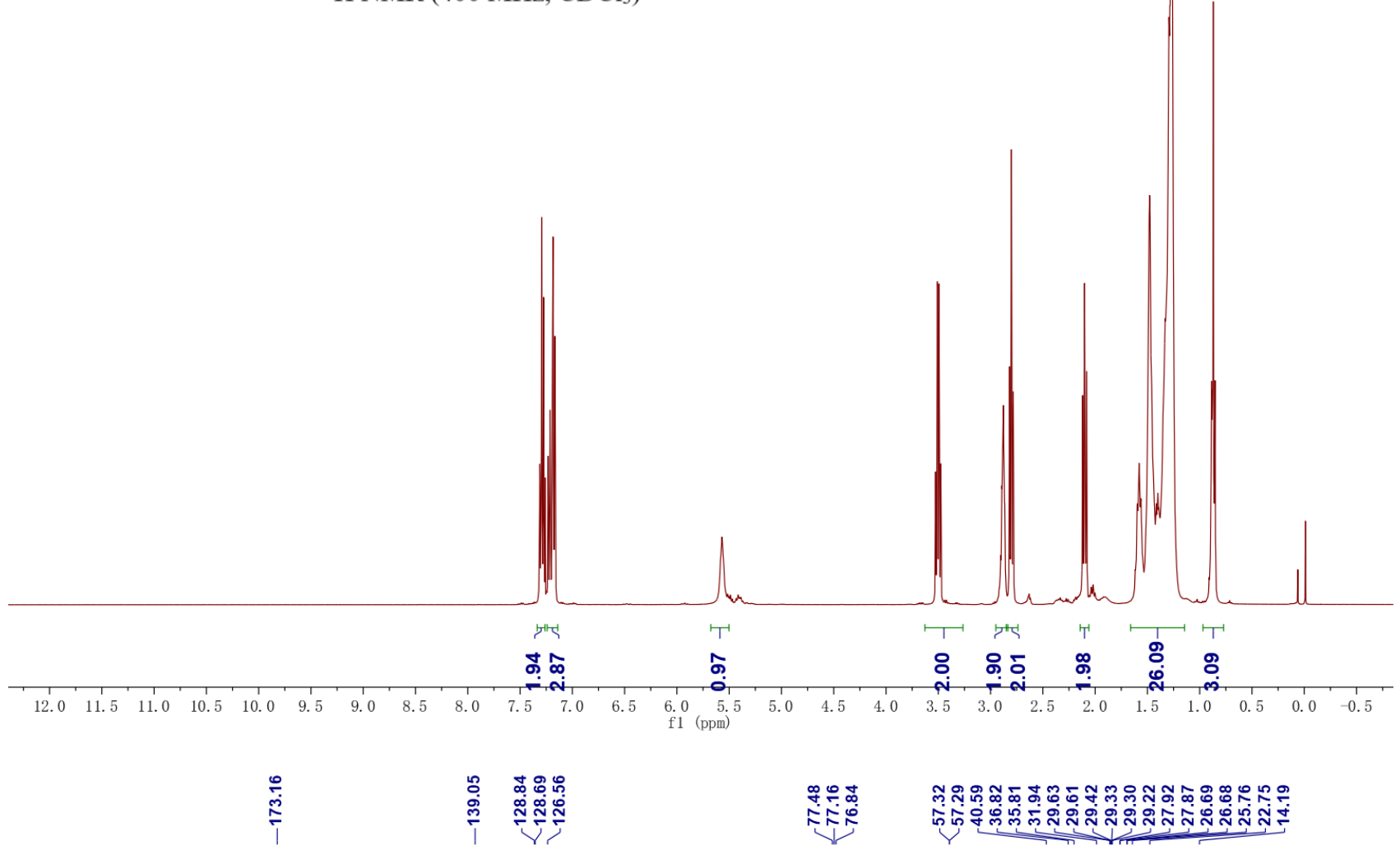<smiles>CCC1OC1CC(=O)NCCc1ccccc1</smiles>

${ }^{13} \mathrm{C}$ NMR (100 MHz, $\mathrm{CDCl}_{3}$ )

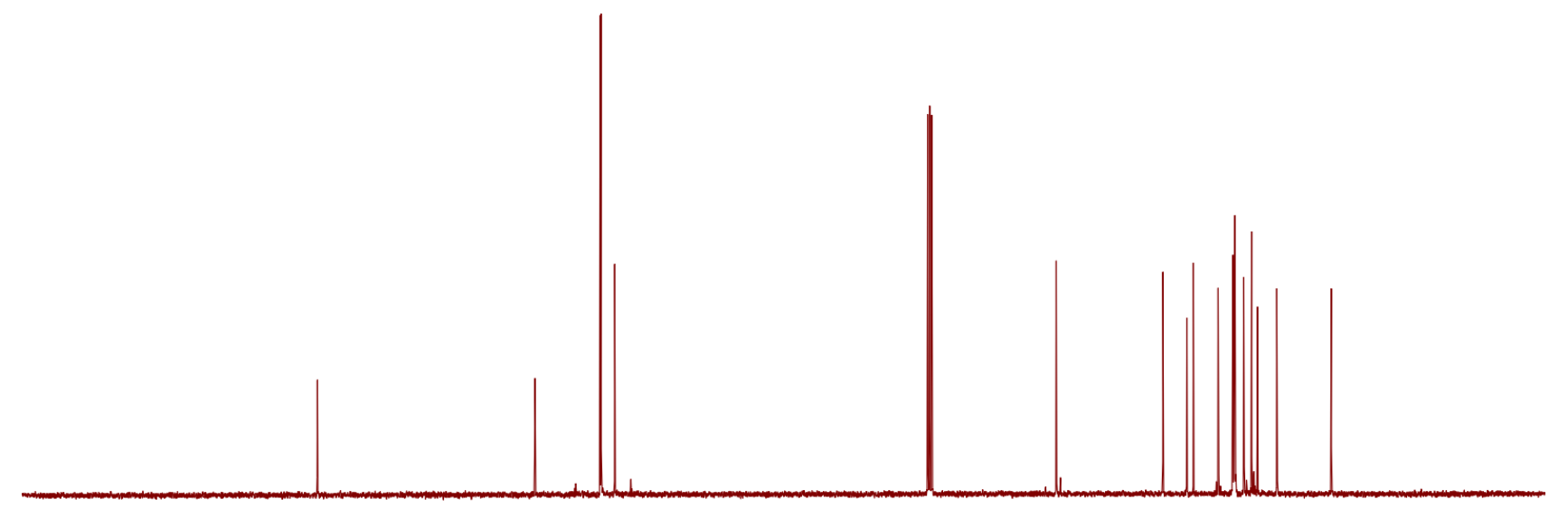

Figure S84. ${ }^{1} \mathrm{H}$ NMR and ${ }^{13} \mathrm{C}\left\{{ }^{1} \mathrm{H}\right\}$ NMR Spectra of 8-(3-octyloxiran-2-yl)- $N$-phenethyloctanamide (7b). 

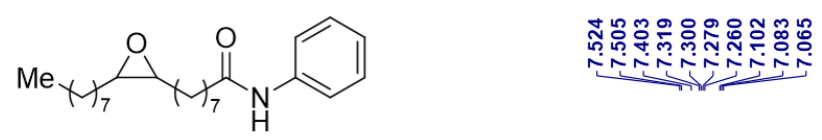

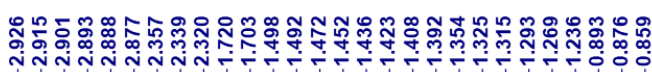

${ }^{1} \mathrm{H}$ NMR (400 MHz, $\mathrm{CDCl}_{3}$ )

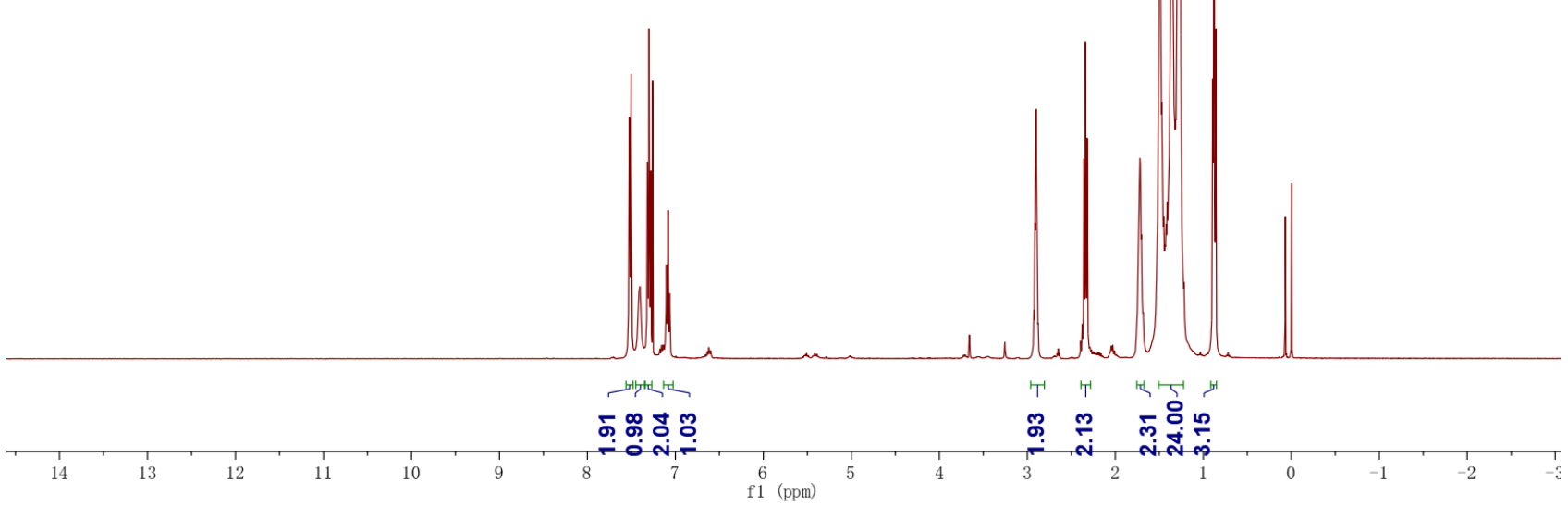<smiles>CCOC1OC1C(=O)Nc1cccc(I)c1</smiles>

${ }^{13} \mathrm{C}$ NMR $\left(100 \mathrm{MHz}, \mathrm{CDCl}_{3}\right)$

$\begin{array}{llllllll}210 & 200 & 190 & 180 & 170 & 160 & 150 & 1\end{array}$

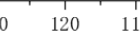

$\mathrm{f} 1 \stackrel{100}{(\mathrm{ppm})}$

Figure S85. ${ }^{1} \mathrm{H}$ NMR and ${ }^{13} \mathrm{C}\left\{{ }^{1} \mathrm{H}\right\}$ NMR Spectra of 8-(3-octyloxiran-2-yl)- $N$-phenyloctanamide (7c). 

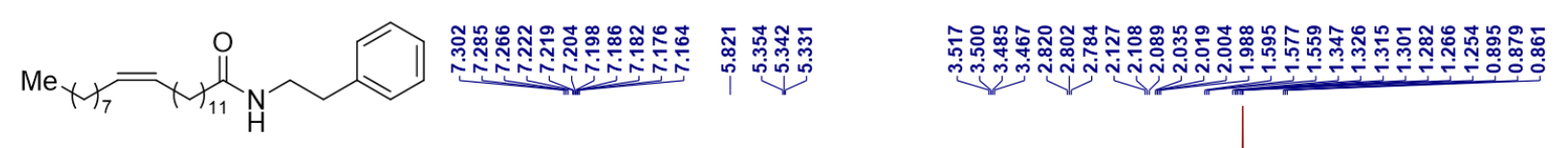

${ }^{1} \mathrm{H} \mathrm{NMR}\left(400 \mathrm{MHz}, \mathrm{CDCl}_{3}\right)$
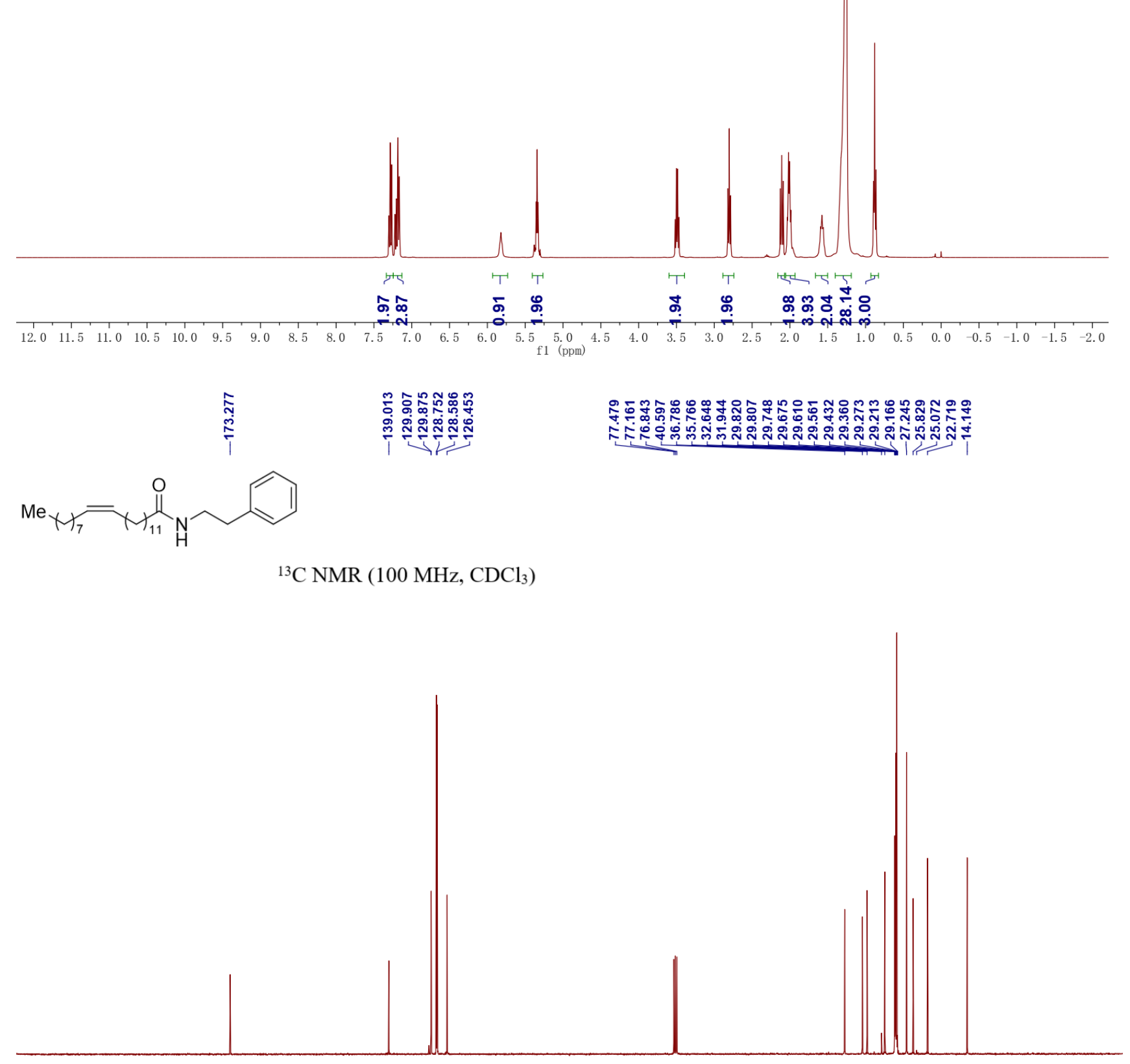

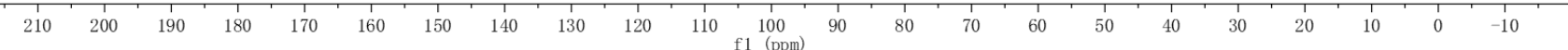

Figure S86. ${ }^{1} \mathrm{H}$ NMR and ${ }^{13} \mathrm{C}\left\{{ }^{1} \mathrm{H}\right\}$ NMR Spectra of (E)- $N$-phenethyldocos-13-enamide (7d). 
<smiles>CC=CCC=CCCCCC(=O)NCCc1ccccc1</smiles>

${ }^{1} \mathrm{H}$ NMR $\left(400 \mathrm{MHz}, \mathrm{CDCl}_{3}\right)$
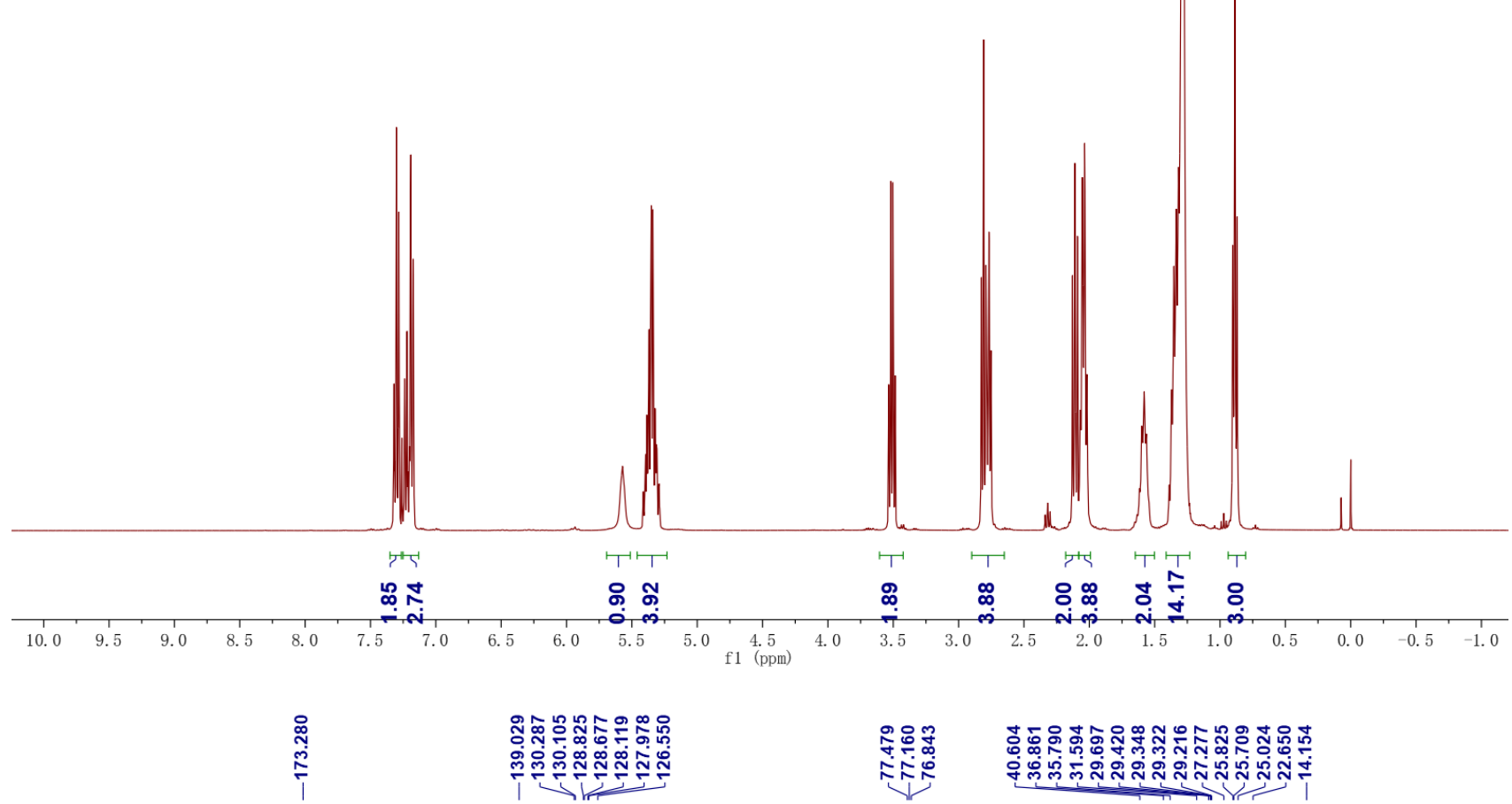

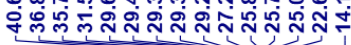<smiles>CC=CCC=CNC(=O)NCCc1ccccc1</smiles>

${ }^{13} \mathrm{C}$ NMR $\left(100 \mathrm{MHz}, \mathrm{CDCl}_{3}\right)$

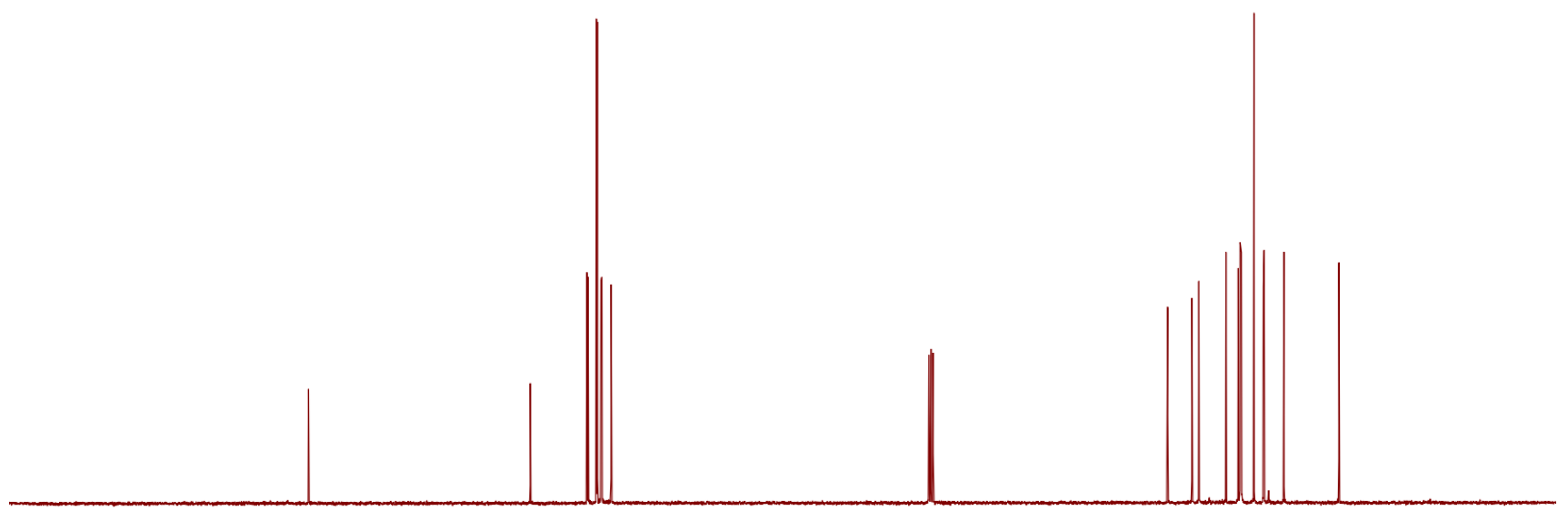

Figure S87. ${ }^{1} \mathrm{H}$ NMR and ${ }^{13} \mathrm{C}\left\{{ }^{1} \mathrm{H}\right\}$ NMR Spectra of (9Z,12E)- $N$-phenethyloctadeca-9,12-dienamide (7e). 
$\mathrm{H}_{2} \mathrm{C}=\mathrm{H}_{8}$

${ }^{1} \mathrm{H} \mathrm{NMR}\left(400 \mathrm{MHz}, \mathrm{CDCl}_{3}\right)$

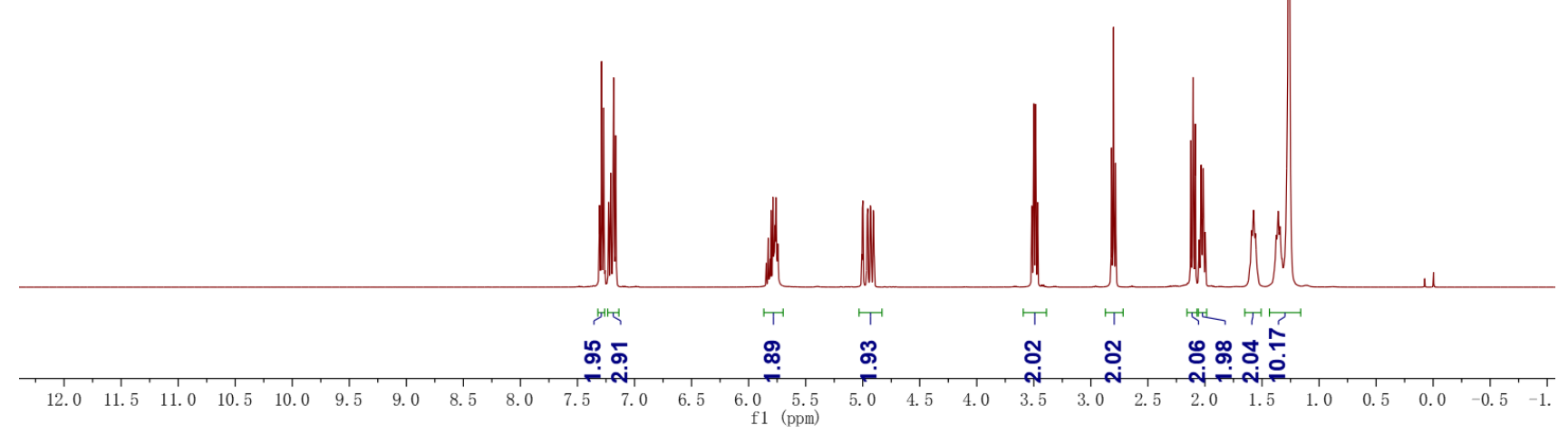<smiles>C=CCC(=O)NCCc1ccccc1</smiles>

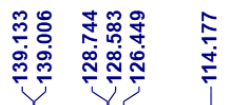

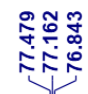

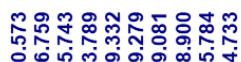

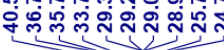

${ }^{13} \mathrm{C} \mathrm{NMR}\left(100 \mathrm{MHz}, \mathrm{CDCl}_{3}\right)$

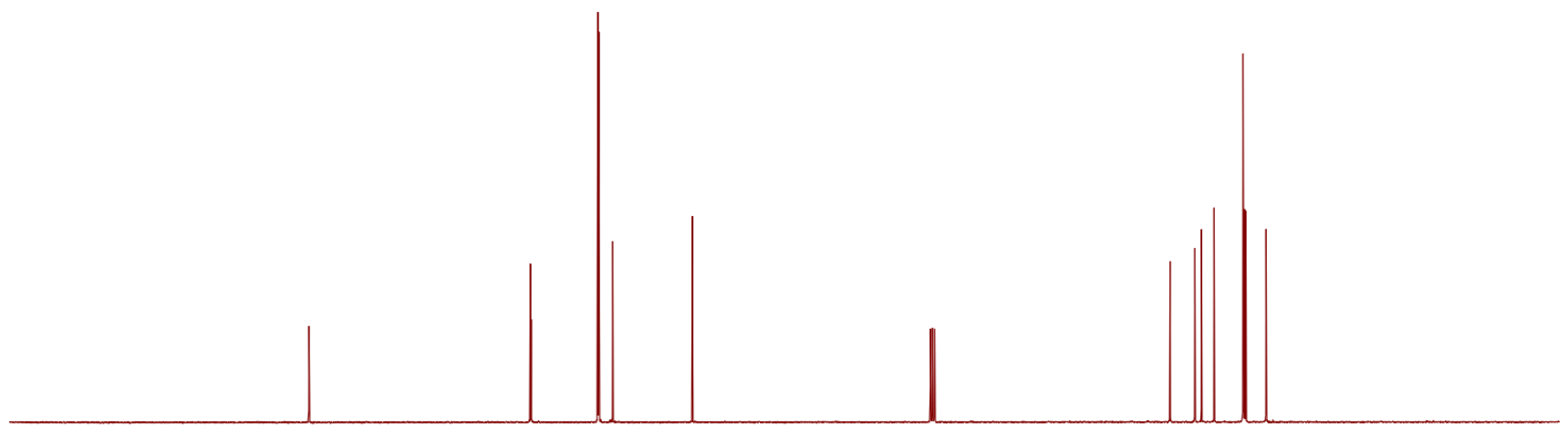

$\begin{array}{llllll}210 & 200 & 190 & 180 & 170 & 160\end{array}$

Figure S88. ${ }^{1} \mathrm{H}$ NMR and ${ }^{13} \mathrm{C}\left\{{ }^{1} \mathrm{H}\right\}$ NMR Spectra of $N$-phenethylundec-10-enamide (7f). 
<smiles>COC(=O)c1ccc(CC(=O)Nc2ccccc2)cc1</smiles>

${ }^{1} \mathrm{H} \mathrm{NMR}\left(400 \mathrm{MHz}, \mathrm{CDCl}_{3}\right)$

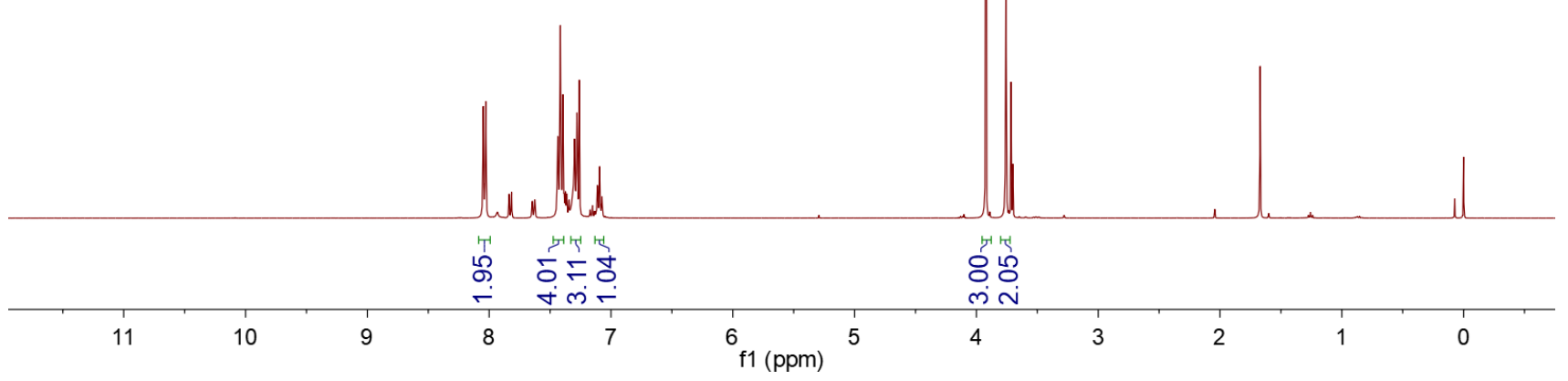

至<smiles>COC(=O)c1ccc(CC(=O)Nc2ccccc2)cc1</smiles>

${ }^{13} \mathrm{C}$ NMR $\left(100 \mathrm{MHz}, \mathrm{CDCl}_{3}\right)$

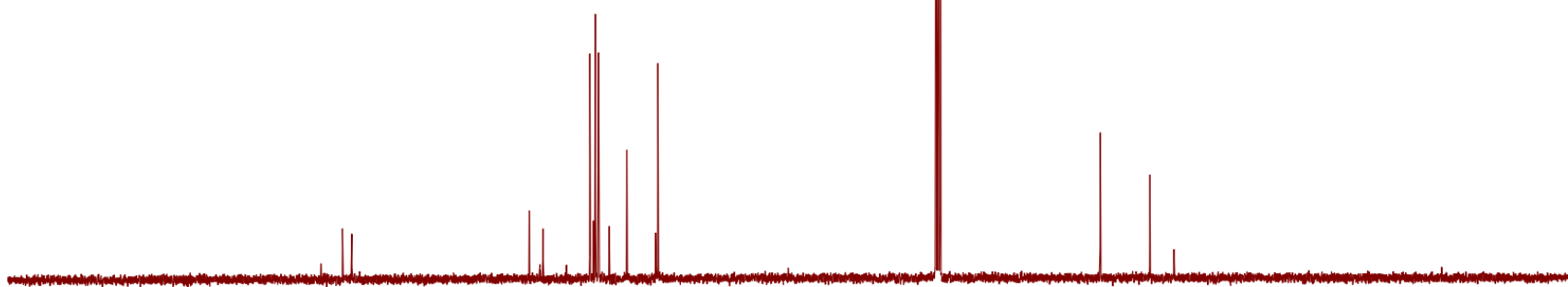

Figure S89. ${ }^{1} \mathrm{H}$ NMR and ${ }^{13} \mathrm{C}\left\{{ }^{1} \mathrm{H}\right\}$ NMR Spectra of methyl 4-(2-oxo-2-(phenylamino)ethyl)benzoate (A). 


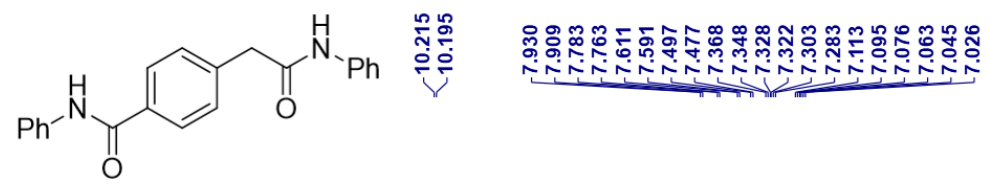

${ }^{1} \mathrm{H}$ NMR (400 MHz, DMSO- $\left.d_{6}\right)$
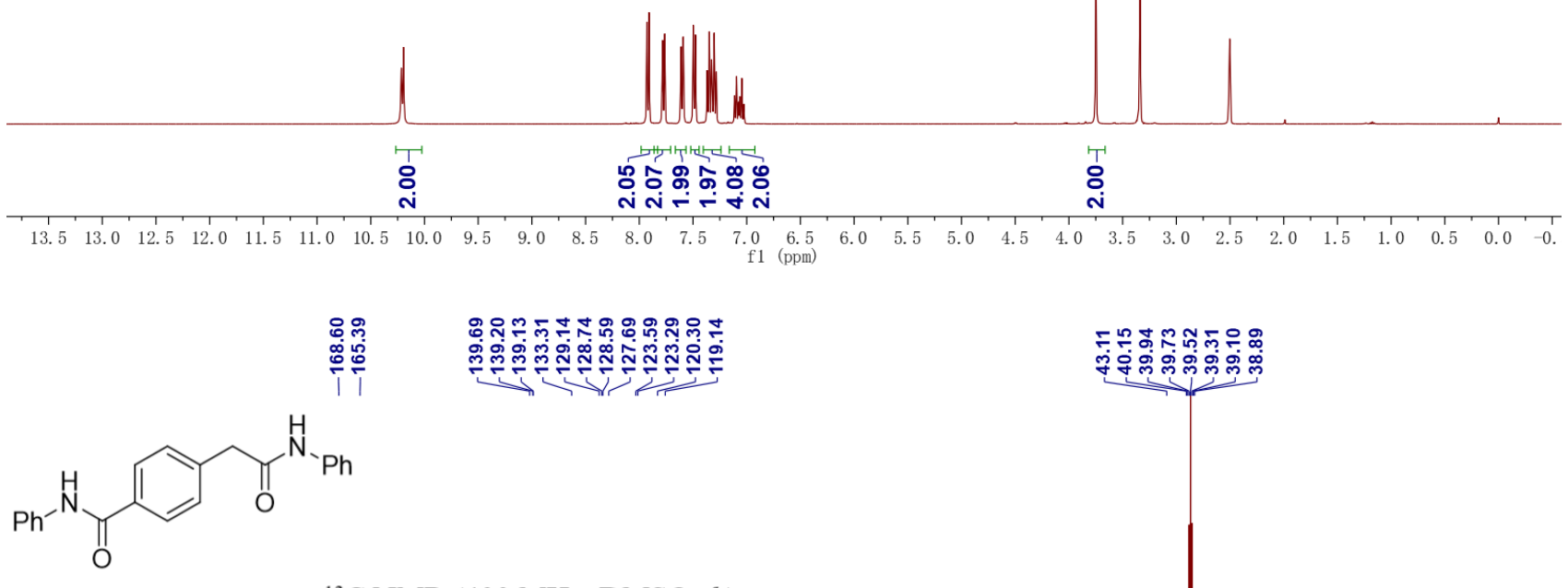

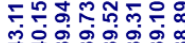

भु일

${ }^{13} \mathrm{C}$ NMR (400 MHz, DMSO- $\left.d_{6}\right)$

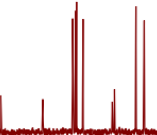

$\begin{array}{llllllllll}210 & 200 & 190 & 180 & 170 & 160 & 150 & 140 & 130 & 120\end{array}$ $\mathrm{f} 1(\mathrm{ppm})$

Figure S90. ${ }^{1} \mathrm{H}$ NMR and ${ }^{13} \mathrm{C}\left\{{ }^{1} \mathrm{H}\right\}$ NMR Spectra of 4-(2-oxo-2-(phenylamino)ethyl)- $N$-phenylbenzamide (B). 
$\mathrm{N}_{\mathrm{H}}^{\mathrm{O}} \mathrm{N}_{\mathrm{H}}$

${ }^{1} \mathrm{H} \mathrm{NMR}\left(400 \mathrm{MHz}, \mathrm{CDCl}_{3}\right)$

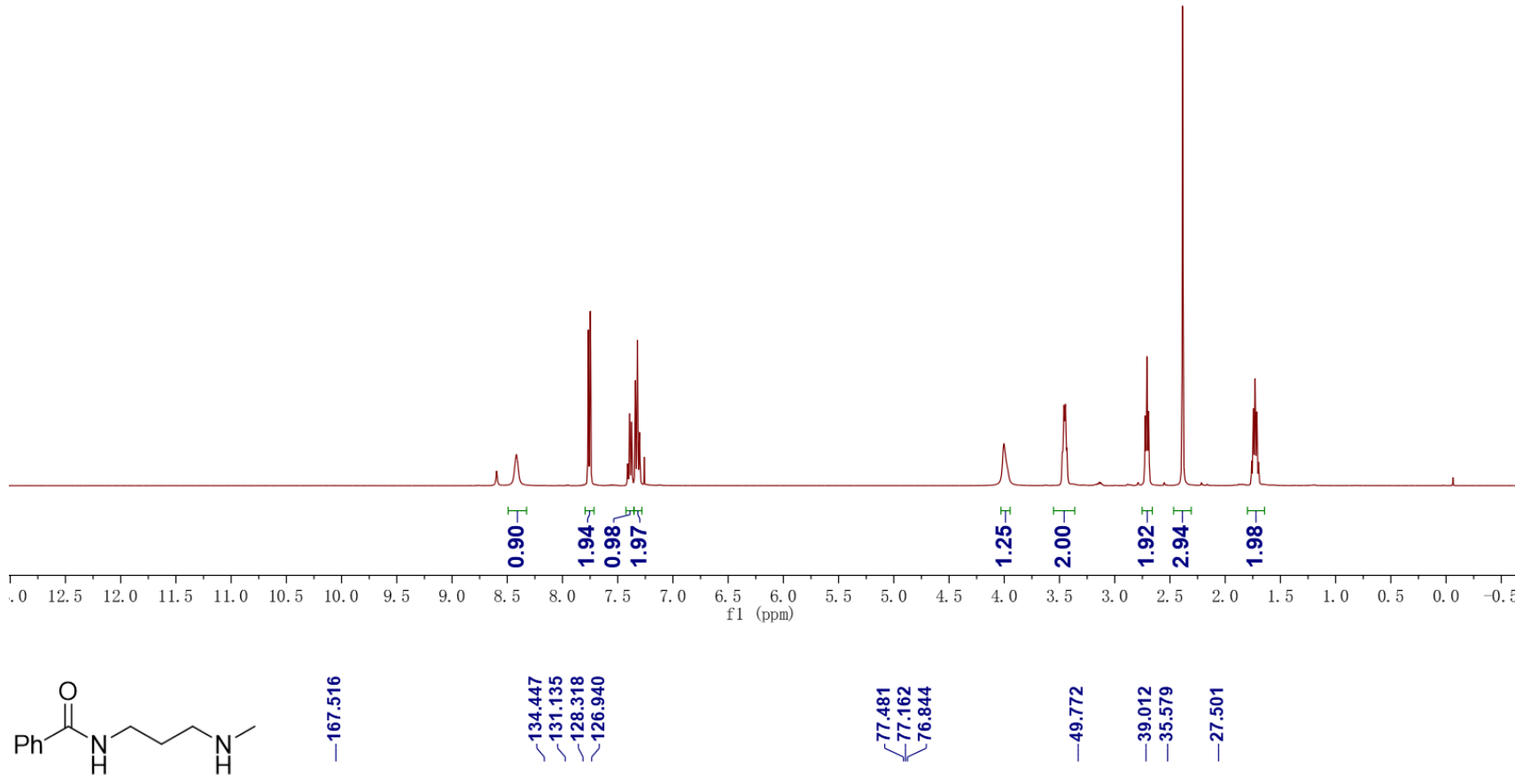

${ }^{13} \mathrm{C} \mathrm{NMR}\left(100 \mathrm{MHz}, \mathrm{CDCl}_{3}\right)$

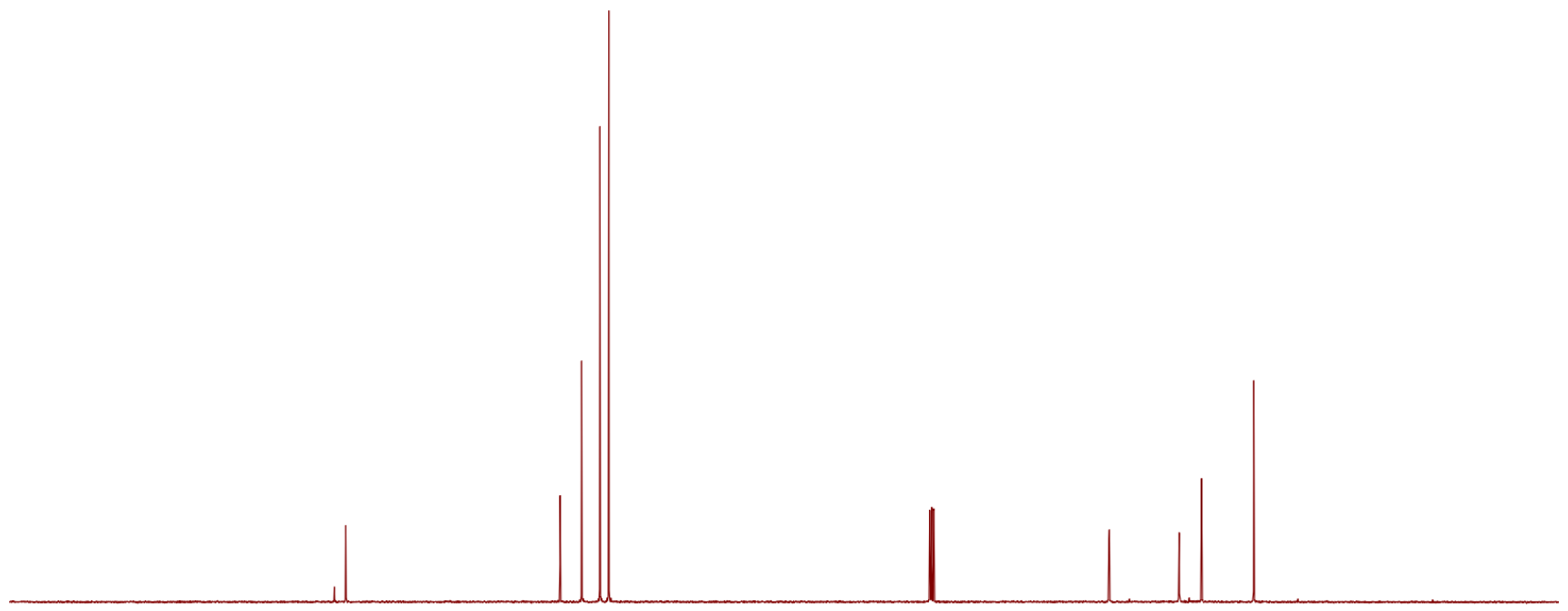

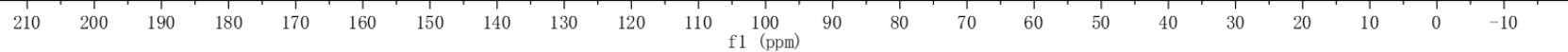

Figure S91. ${ }^{1} \mathrm{H}$ NMR and ${ }^{13} \mathrm{C}\left\{{ }^{1} \mathrm{H}\right\}$ NMR Spectra of N-(3-(methylamino)propyl)benzamide (C). 
<smiles>CC(CO)NC(=O)c1ccccc1</smiles>

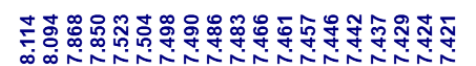

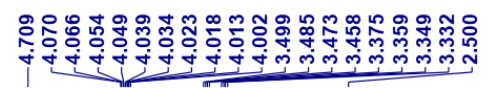

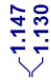

${ }^{1} \mathrm{H}$ NMR (400 MHz, $\left.\mathrm{CDCl}_{3}\right)$

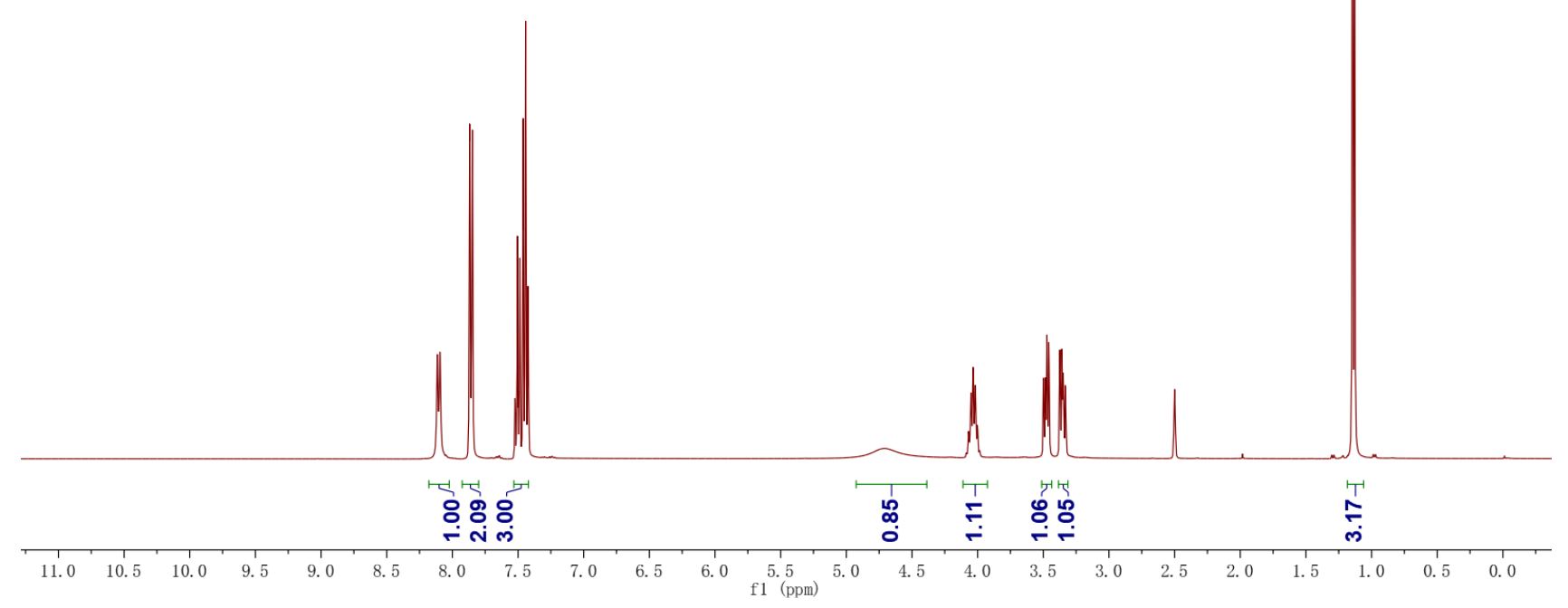

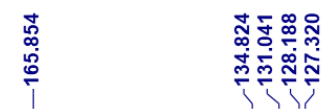

${ }^{13} \mathrm{C}$ NMR (400 MHz, DMSO- $\left.d_{6}\right)$

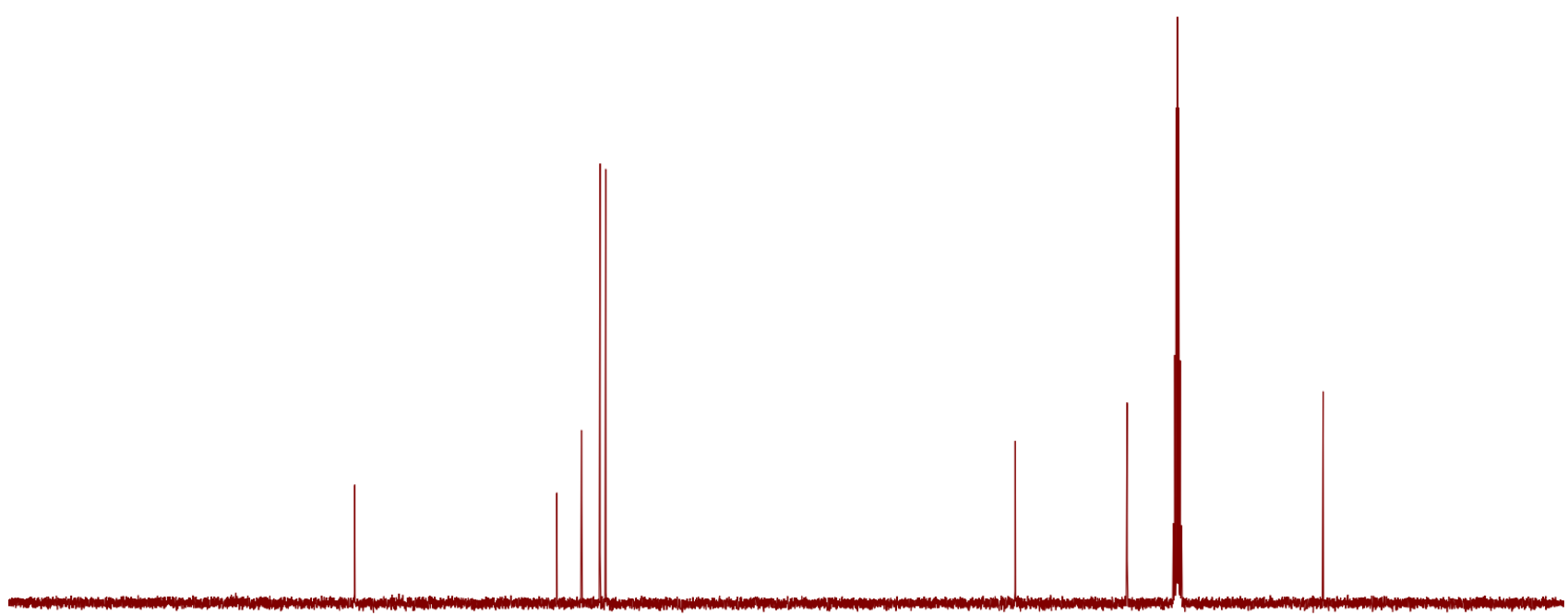

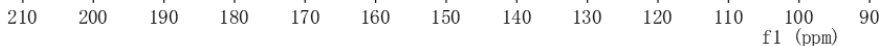

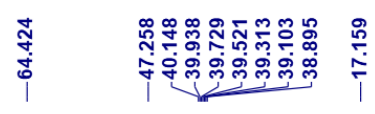

Figure S92. ${ }^{1} \mathrm{H}$ NMR and ${ }^{13} \mathrm{C}\left\{{ }^{1} \mathrm{H}\right\}$ NMR Spectra of N-(1-hydroxypropan-2-yl)benzamide (D). 
<smiles>COc1cc(C(=O)NCc2ccc(OCCN(C)C)cc2)cc(OC)c1OC</smiles>

${ }^{1} \mathrm{H}$ NMR (400 MHz, $\left.\mathrm{CDCl}_{3}\right)$

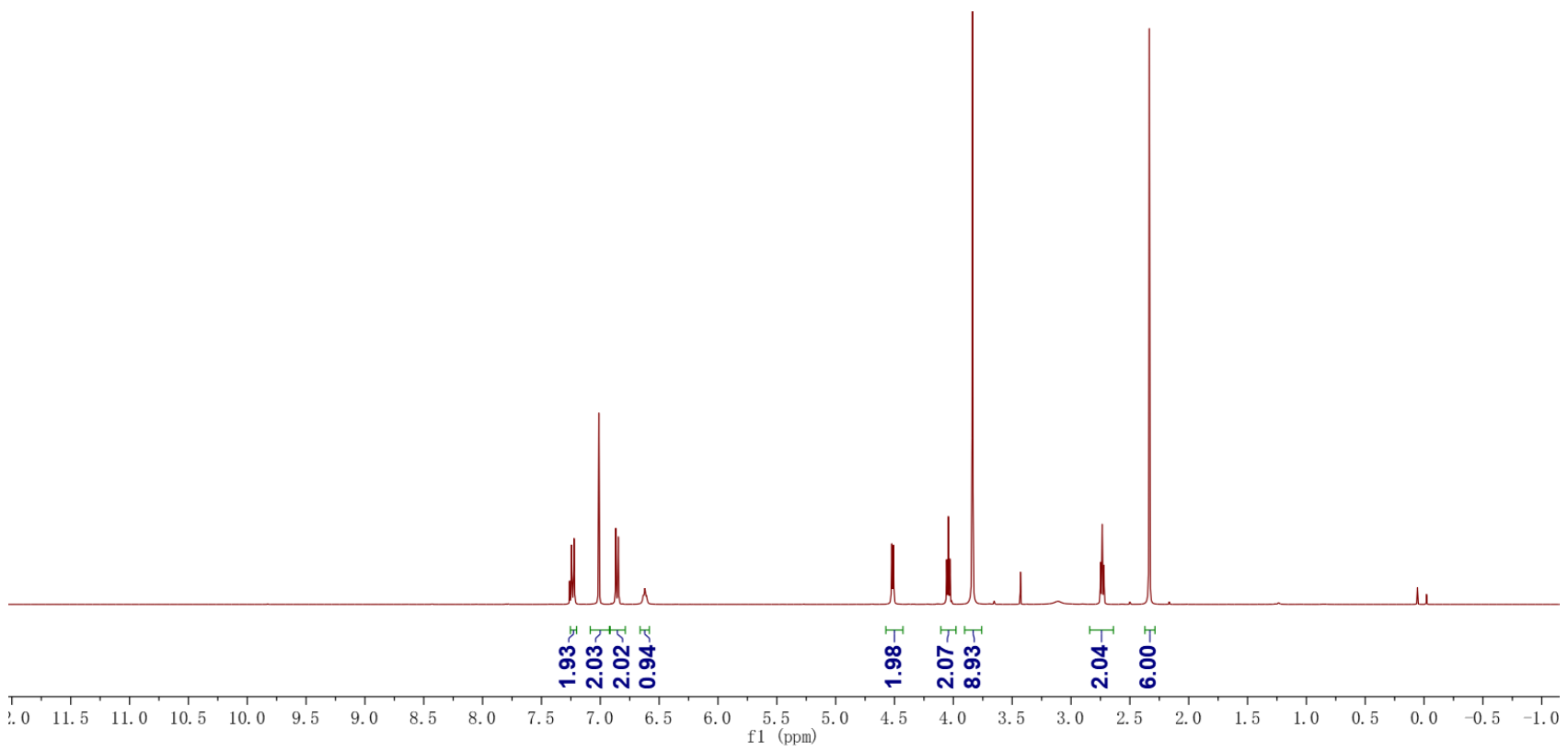<smiles>COc1cc(C(=O)NCc2ccc(OCCN(C)C)cc2)cc(OC)c1OC</smiles>

${ }^{13} \mathrm{C} \mathrm{NMR}\left(100 \mathrm{MHz}, \mathrm{CDCl}_{3}\right)$

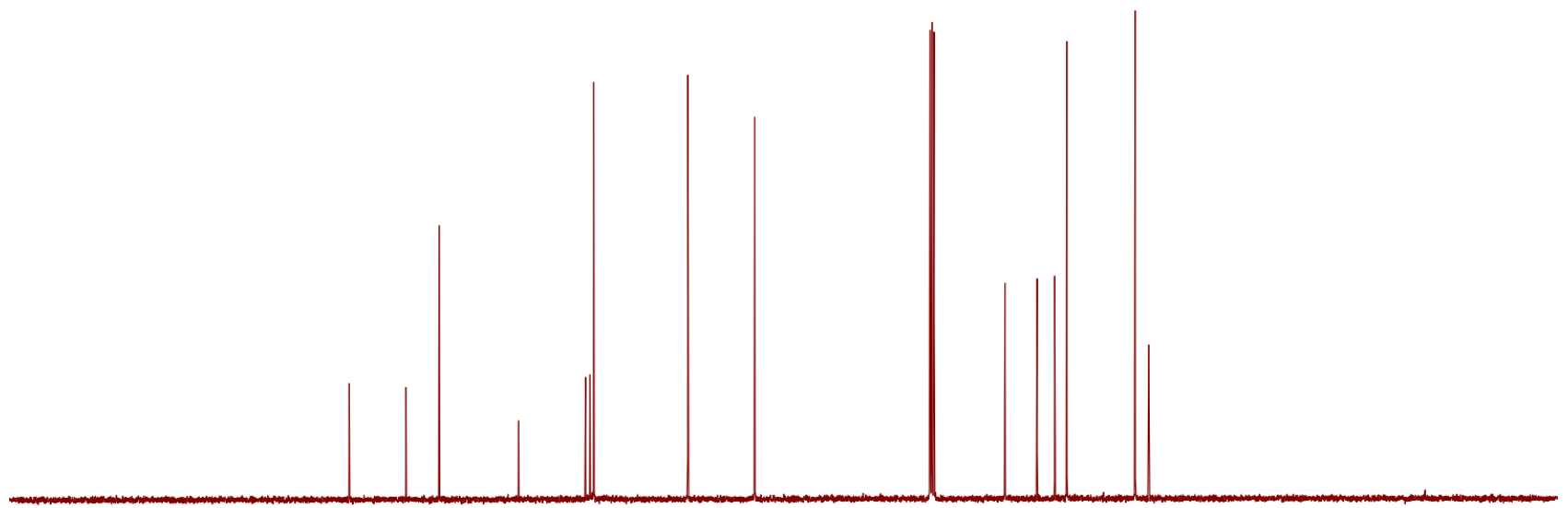

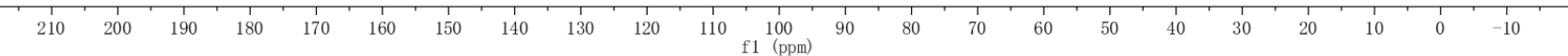

Figure S93. ${ }^{1} \mathrm{H}$ NMR and ${ }^{13} \mathrm{C}\left\{{ }^{1} \mathrm{H}\right\}$ NMR Spectra of $N$-(4-(2-(dimethylamino)ethoxy)benzyl)-3,4,5trimethoxybenzamide $(\mathbf{E})$. 
<smiles>COc1ccc(C(=O)NCc2ccc(OCCN(C)C)cc2)cc1OC</smiles>

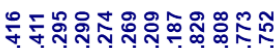

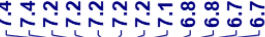

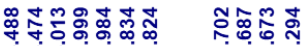

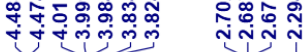

${ }^{1} \mathrm{H} \mathrm{NMR}\left(400 \mathrm{MHz}, \mathrm{CDCl}_{3}\right)$
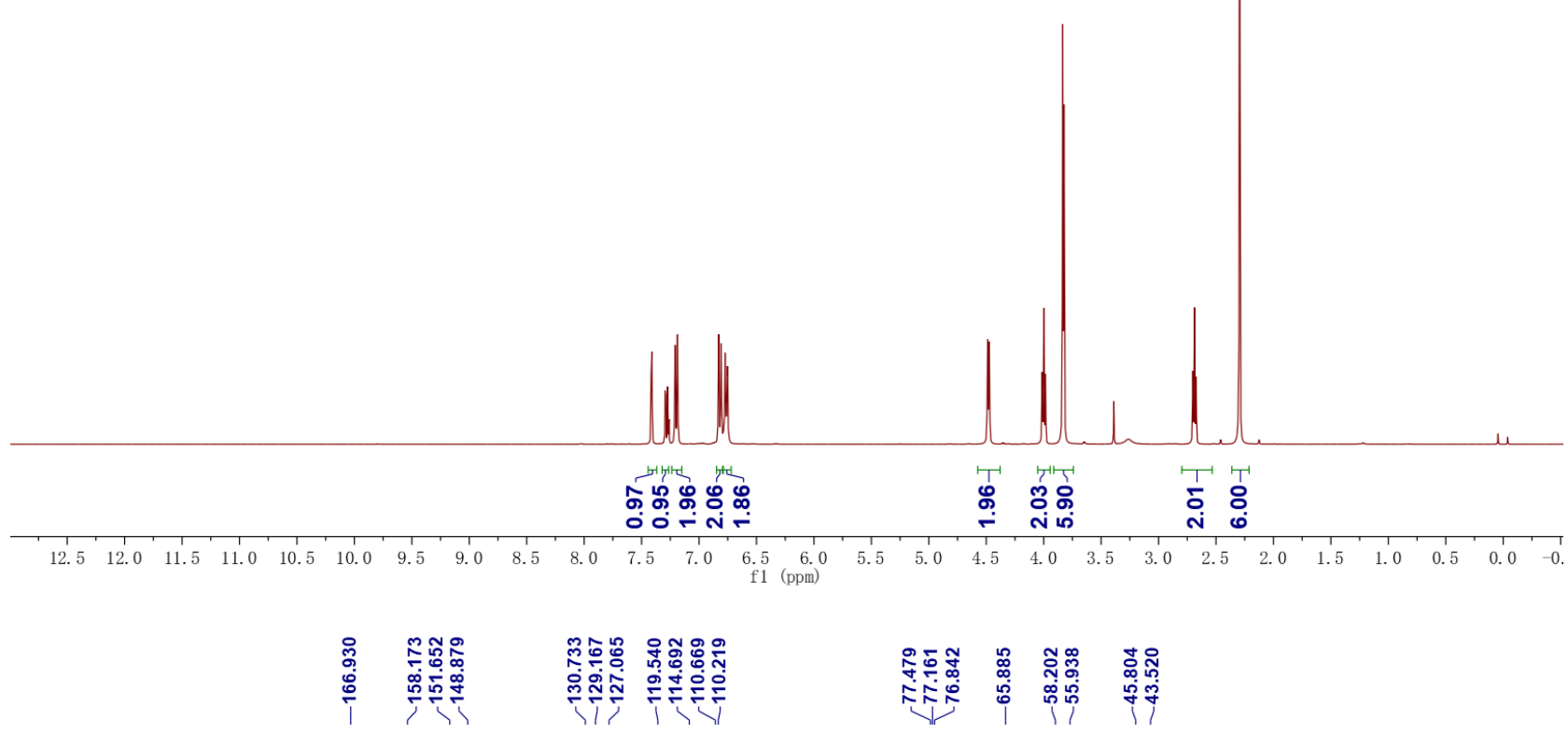<smiles>COc1ccc(C(=O)NCc2ccc(OCCN(C)C)cc2)cc1OC</smiles>

${ }^{13} \mathrm{C} \mathrm{NMR}\left(100 \mathrm{MHz}, \mathrm{CDCl}_{3}\right)$

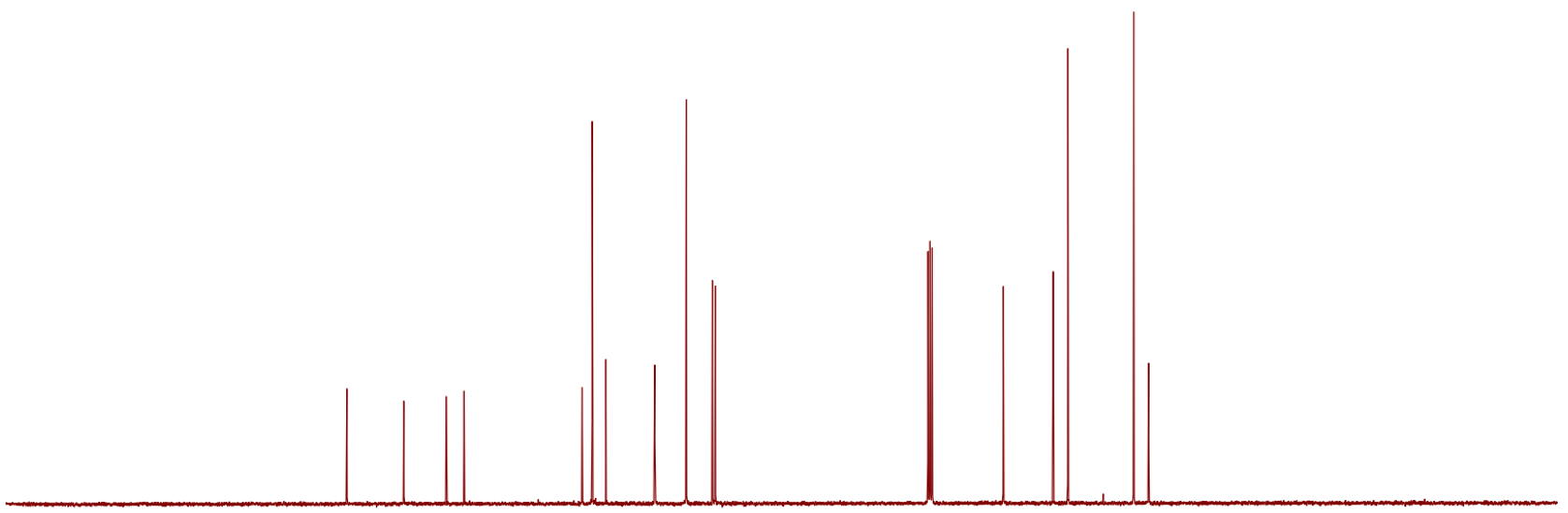

$\begin{array}{llllllllllllllllllllllllllllll}1 & 200 & 190 & 180 & 170 & 160 & 150 & 140 & 130 & 120 & 110 & 100 & 90 & 80 & 70 & 60 & 50 & 40 & 30 & 20 & 10 & 0 & -10\end{array}$

Figure S94. ${ }^{1} \mathrm{H}$ NMR and ${ }^{13} \mathrm{C}\left\{{ }^{1} \mathrm{H}\right\}$ NMR Spectra of $N$-(4-(2-(dimethylamino)ethoxy)benzyl)-3,4dimethoxybenzamide $(\mathbf{F})$. 


\section{The Energies, Geometrical Coordinates, and Frequencies of the Listed Compounds}

\begin{tabular}{|c|c|c|c|c|}
\hline SPs & $\begin{array}{l}\text { Correction to } \\
\text { Gibbs energy }^{\mathrm{a}}\end{array}$ & $\begin{array}{c}\text { Single-point } \\
\text { energy }^{\mathrm{b}}\end{array}$ & Gibbs energy $^{c}$ & $\begin{array}{l}\text { Number of } \\
\text { imaginary } \\
\text { frequencies }\end{array}$ \\
\hline $\mathbf{R 1}$ & 0.109327 & -460.280352 & -460.17103 & 0 \\
\hline Cat & 0.246472 & -1201.146648 & -1200.9002 & 0 \\
\hline 1 & 0.222876 & -560.266689 & -560.04381 & 0 \\
\hline 2-ts & 0.223257 & -560.266673 & -560.04342 & 1 \\
\hline 3 & 0.226198 & -560.272269 & -560.04607 & 0 \\
\hline 4 & 0.621356 & -2221.754399 & -2221.133 & 0 \\
\hline 5-ts & 0.629793 & -2221.7327 & -2221.1029 & 1 \\
\hline 6 & 0.628952 & -2221.733163 & -2221.1042 & 0 \\
\hline 7-ts & 0.625125 & -2221.710085 & -2221.085 & 1 \\
\hline $\mathbf{P}$ & 0.195485 & -671.541648 & -671.34616 & 0 \\
\hline 8 & 0.137226 & -349.007277 & -348.87005 & 0 \\
\hline 9-ts & 0.135852 & -349.006964 & -348.87111 & 1 \\
\hline 10 & 0.137273 & -349.007673 & -348.8704 & 0 \\
\hline 11 & 0.373737 & -1661.421915 & -1661.0482 & 0 \\
\hline 12 & 0.370771 & -1661.275214 & -1660.9044 & 0 \\
\hline 4 & 0.612974 & -2383.987582 & -2383.374608 & 0 \\
\hline 7'-ts & 0.620813 & -2383.942306 & -2383.321493 & 1 \\
\hline $4 "$ & 0.376982 & -1705.275557 & -1704.898575 & 0 \\
\hline 7 ''-ts & 0.38309 & -1705.215491 & -1704.832401 & 1 \\
\hline $4 n$ & 0.62696 & -2210.17 & -2209.54 & 0 \\
\hline 7n-ts & 0.633853 & -2210.12 & -2209.49 & 1 \\
\hline $4 n^{\prime \prime}$ & 0.37976 & -1691.74 & -1691.36 & 0 \\
\hline $7 n$ "'-ts & 0.387177 & -1691.69 & -1691.3 & 1 \\
\hline
\end{tabular}

${ }^{a}$ Calculated at the level of B3LYP/6-31G(d, p)/SDD (SMD, solvent=toluene).

${ }^{\mathrm{b}}$ Calculated at the level of B3LYP-D3/6-311+G(d, p)/SDD (SMD, solvent=toluene).

${ }^{c}$ Sum of correction to Gibbs energy and single-point energy.

\section{1}

Zero-point correction $=0.265491$

Thermal correction to Energy $=0.280597$

Thermal correction to Enthalpy $=0.281541$

Thermal correction to Gibbs Free Energy $=0.222876$

Sum of electronic and zero-point Energies $=-559.824386$

Sum of electronic and thermal Energies $=-559.809280$

Sum of electronic and thermal Enthalpies= -559.808335

Sum of electronic and thermal Free Energies $=-559.867001$

Cartesian coordinates

$\begin{array}{lrrr}\mathrm{C} & -1.157441 & -0.556914 & -0.064451 \\ \mathrm{C} & -2.398311 & -1.175381 & -0.050597 \\ \mathrm{C} & -3.602369 & -0.446326 & -0.005678 \\ \mathrm{C} & -3.492425 & 0.948763 & 0.018728 \\ \mathrm{C} & -2.255809 & 1.594279 & 0.007130 \\ \mathrm{C} & -1.044161 & 0.860983 & -0.028389 \\ \mathrm{H} & -0.231804 & -1.122292 & -0.135583 \\ \mathrm{H} & -2.441170 & -2.264034 & -0.086570 \\ \mathrm{H} & -4.399280 & 1.552613 & 0.045348 \\ \mathrm{H} & -2.214831 & 2.682543 & 0.022166 \\ \mathrm{C} & -4.942633 & -1.143649 & 0.025028 \\ \mathrm{H} & -5.145894 & -1.624712 & 0.992989 \\ \mathrm{H} & -5.762843 & -0.441081 & -0.160556 \\ \mathrm{H} & -5.012459 & -1.932558 & -0.735168\end{array}$




$\begin{array}{lrrr}\mathrm{N} & 0.193036 & 1.435453 & -0.012189 \\ \mathrm{H} & 0.206140 & 2.430307 & -0.200466 \\ \mathrm{C} & 4.011104 & -1.497496 & -0.478516 \\ \mathrm{C} & 3.207736 & -0.246831 & -0.026593 \\ \mathrm{H} & 3.403310 & -2.396972 & -0.322508 \\ \mathrm{H} & 4.223440 & -1.429703 & -1.552627 \\ \mathrm{H} & 4.965226 & -1.630239 & 0.053518 \\ \mathrm{C} & 4.098161 & 1.010203 & -0.258263 \\ \mathrm{C} & 2.933787 & -0.371734 & 1.500453 \\ \mathrm{H} & 3.568305 & 1.909919 & 0.079564 \\ \mathrm{H} & 5.067352 & 0.976212 & 0.262726 \\ \mathrm{H} & 4.292020 & 1.127344 & -1.331461 \\ \mathrm{H} & 3.847224 & -0.443958 & 2.110274 \\ \mathrm{H} & 2.358833 & 0.494115 & 1.848453 \\ \mathrm{H} & 2.326512 & -1.264286 & 1.693683 \\ \mathrm{O} & 2.048227 & -0.166409 & -0.750365 \\ \mathrm{H} & 1.067018 & 0.804517 & -0.328639\end{array}$

2-ts

Zero-point correction $=0.264650$

Thermal correction to Energy $=0.279039$

Thermal correction to Enthalpy= 0.279983

Thermal correction to Gibbs Free Energy $=0.223257$

Sum of electronic and zero-point Energies $=-559.825239$

Sum of electronic and thermal Energies $=-559.810851$

Sum of electronic and thermal Enthalpies $=-559.809906$

Sum of electronic and thermal Free Energies $=-559.866632$

Cartesian coordinates

$\begin{array}{lrrr}\mathrm{C} & -1.177189 & -0.589253 & -0.083818 \\ \mathrm{C} & -2.431301 & -1.179885 & -0.064799 \\ \mathrm{C} & -3.618768 & -0.425011 & -0.005138 \\ \mathrm{C} & -3.476717 & 0.967216 & 0.027943 \\ \mathrm{C} & -2.226422 & 1.585010 & 0.010564 \\ \mathrm{C} & -1.028655 & 0.826607 & -0.038345 \\ \mathrm{H} & -0.268221 & -1.179483 & -0.163353 \\ \mathrm{H} & -2.498408 & -2.267156 & -0.107442 \\ \mathrm{H} & -4.369555 & 1.591135 & 0.067468 \\ \mathrm{H} & -2.161701 & 2.672182 & 0.032501 \\ \mathrm{C} & -4.973466 & -1.092907 & 0.037992 \\ \mathrm{H} & -5.188136 & -1.550967 & 1.014969 \\ \mathrm{H} & -5.778883 & -0.377356 & -0.162223 \\ \mathrm{H} & -5.059485 & -1.895311 & -0.706203 \\ \mathrm{~N} & 0.218775 & 1.371826 & -0.031601 \\ \mathrm{H} & 0.244777 & 2.373265 & -0.182962 \\ \mathrm{C} & 4.140320 & -1.358650 & -0.569770 \\ \mathrm{C} & 3.213952 & -0.240542 & -0.022481 \\ \mathrm{H} & 3.607769 & -2.317078 & -0.547360 \\ \mathrm{H} & 4.390071 & -1.149610 & -1.617045 \\ \mathrm{H} & 5.078780 & -1.471713 & -0.006569 \\ \mathrm{C} & 3.990892 & 1.106950 & -0.081284 \\ \mathrm{C} & 2.895413 & -0.555260 & 1.467100 \\ \mathrm{H} & 3.370577 & 1.914174 & 0.327565 \\ \mathrm{H} & 4.941602 & 1.100374 & 0.473031 \\ \mathrm{H} & 4.206640 & 1.355682 & -1.127492 \\ \mathrm{H} & 3.789601 & -0.619145 & 2.105461 \\ \mathrm{H} & 2.236670 & 0.217541 & 1.879006 \\ \mathrm{H} & 2.361025 & -1.510475 & 1.536203 \\ \mathrm{O} & 2.076439 & -0.184762 & -0.787223 \\ \mathrm{H} & 1.105591 & 0.723604 & -0.364336\end{array}$

3

Zero-point correction $=0.268013$

Thermal correction to Energy $=0.282839$ 
Thermal correction to Enthalpy $=0.283783$

Thermal correction to Gibbs Free Energy= 0.226198

Sum of electronic and zero-point Energies $=-559.827972$

Sum of electronic and thermal Energies $=-559.813146$

Sum of electronic and thermal Enthalpies $=-559.812202$

Sum of electronic and thermal Free Energies $=-559.869787$

Cartesian coordinates

$\begin{array}{lrrr}\mathrm{C} & -1.274140 & -0.638142 & -0.398631 \\ \mathrm{C} & -2.544385 & -1.171081 & -0.255222 \\ \mathrm{C} & -3.668650 & -0.378846 & 0.056042 \\ \mathrm{C} & -3.431151 & 0.995146 & 0.209256 \\ \mathrm{C} & -2.164665 & 1.555128 & 0.070420 \\ \mathrm{C} & -1.006372 & 0.765641 & -0.237652 \\ \mathrm{H} & -0.433224 & -1.280912 & -0.648752 \\ \mathrm{H} & -2.678051 & -2.245605 & -0.391190 \\ \mathrm{H} & -4.270114 & 1.652110 & 0.444900 \\ \mathrm{H} & -2.038564 & 2.631105 & 0.194992 \\ \mathrm{C} & -5.041076 & -0.981813 & 0.239928 \\ \mathrm{H} & -5.145188 & -1.538399 & 1.184934 \\ \mathrm{H} & -5.817860 & -0.207609 & 0.244884 \\ \mathrm{H} & -5.298126 & -1.688269 & -0.561690 \\ \mathrm{~N} & 0.245365 & 1.236091 & -0.380729 \\ \mathrm{H} & 0.251273 & 2.250168 & -0.258275 \\ \mathrm{C} & 4.528822 & -1.000229 & -0.372426 \\ \mathrm{C} & 3.280599 & -0.221517 & 0.068656 \\ \mathrm{H} & 4.247090 & -2.001538 & -0.716434 \\ \mathrm{H} & 5.021054 & -0.488682 & -1.207209 \\ \mathrm{H} & 5.254596 & -1.107694 & 0.442942 \\ \mathrm{C} & 3.678843 & 1.198421 & 0.523279 \\ \mathrm{C} & 2.580264 & -0.959628 & 1.227775 \\ \mathrm{H} & 2.787709 & 1.751258 & 0.838413 \\ \mathrm{H} & 4.390498 & 1.185641 & 1.359345 \\ \mathrm{H} & 4.137383 & 1.743486 & -0.309699 \\ \mathrm{H} & 3.224986 & -1.053113 & 2.111841 \\ \mathrm{H} & 1.673019 & -0.420198 & 1.515354 \\ \mathrm{H} & 2.286389 & -1.966560 & 0.910598 \\ \mathrm{O} & 2.433095 & -0.153422 & -1.058597 \\ \mathrm{H} & 1.596264 & 0.381062 & -0.809625\end{array}$

Cat

Zero-point correction $=0.302944$

Thermal correction to Energy $=0.327643$

Thermal correction to Enthalpy $=0.328587$

Thermal correction to Gibbs Free Energy $=0.246472$

Sum of electronic and zero-point Energies $=-1200.510297$

Sum of electronic and thermal Energies $=-1200.485598$

Sum of electronic and thermal Enthalpies $=-1200.484653$

Sum of electronic and thermal Free Energies= -1200.566769

Cartesian coordinates

$\begin{array}{lrrr}\mathrm{C} & -2.179412 & 2.571697 & 0.480195 \\ \mathrm{C} & -2.074840 & 3.909710 & 0.127824 \\ \mathrm{C} & -0.828541 & 4.391603 & -0.267987 \\ \mathrm{C} & 0.266567 & 3.535093 & -0.271495 \\ \mathrm{C} & 0.078414 & 2.209677 & 0.126467 \\ \mathrm{~N} & -1.132463 & 1.725875 & 0.473933 \\ \mathrm{H} & -0.704410 & 5.424668 & -0.576426 \\ \mathrm{H} & -2.952779 & 4.544720 & 0.150188 \\ \mathrm{H} & 1.230045 & 3.888440 & -0.601200 \\ \mathrm{C} & 2.489017 & 1.356660 & -0.014000 \\ \mathrm{C} & 0.744316 & -0.051573 & 0.524796 \\ \mathrm{C} & 2.987770 & 0.061573 & 0.205568 \\ \mathrm{C} & 3.361079 & 2.401891 & -0.329221 \\ \mathrm{C} & 4.343775 & -0.239676 & 0.097400\end{array}$




$\begin{array}{lrrr}\mathrm{C} & 4.720073 & 2.102069 & -0.436666 \\ \mathrm{H} & 3.033172 & 3.420491 & -0.479175 \\ \mathrm{C} & 5.206717 & 0.803966 & -0.231572 \\ \mathrm{H} & 4.714932 & -1.245399 & 0.258914 \\ \mathrm{H} & 5.412661 & 2.900016 & -0.684816 \\ \mathrm{H} & 6.269702 & 0.607305 & -0.327514 \\ \mathrm{~N} & 1.098977 & 1.247701 & 0.189609 \\ \mathrm{~N} & 1.897602 & -0.750968 & 0.542107 \\ \mathrm{C} & 2.035247 & -2.181018 & 0.844815 \\ \mathrm{H} & 2.957413 & -2.301404 & 1.422370 \\ \mathrm{H} & 1.211579 & -2.458224 & 1.501520 \\ \mathrm{C} & 2.061708 & -3.062301 & -0.409543 \\ \mathrm{H} & 1.129509 & -2.918712 & -0.965725 \\ \mathrm{H} & 2.874558 & -2.730554 & -1.066553 \\ \mathrm{C} & 2.245208 & -4.539531 & -0.053733 \\ \mathrm{H} & 1.423126 & -4.904553 & 0.571442 \\ \mathrm{H} & 2.269147 & -5.154236 & -0.958535 \\ \mathrm{H} & 3.182112 & -4.711977 & 0.489521 \\ \mathrm{H} & -3.132653 & 2.148059 & 0.770825 \\ \mathrm{Mn} & -1.226083 & -0.344671 & 0.842441 \\ \mathrm{I} & -1.524152 & -0.703529 & -1.970293 \\ \mathrm{C} & -3.067668 & -0.383754 & 0.900405 \\ \mathrm{O} & -4.219738 & -0.434512 & 0.954067 \\ \mathrm{C} & -1.050675 & -0.125561 & 2.610671 \\ \mathrm{O} & -0.924915 & 0.015377 & 3.754812 \\ \mathrm{C} & -1.214205 & -2.153881 & 0.969824 \\ \mathrm{O} & -1.265607 & -3.306233 & 1.063326\end{array}$

\section{R1}

Zero-point correction $=0.143757$

Thermal correction to Energy $=0.152568$

Thermal correction to Enthalpy $=0.153513$

Thermal correction to Gibbs Free Energy= 0.109327

Sum of electronic and zero-point Energies $=-460.006568$

Sum of electronic and thermal Energies $=-459.997756$

Sum of electronic and thermal Enthalpies $=-459.996812$

Sum of electronic and thermal Free Energies $=-460.040998$

Cartesian coordinates

$\begin{array}{lrrr}\mathrm{C} & -0.752052 & -1.180703 & -0.001592 \\ \mathrm{C} & -2.131776 & -1.376451 & -0.000926 \\ \mathrm{C} & -2.995737 & -0.278785 & 0.000517 \\ \mathrm{C} & -2.478907 & 1.019414 & 0.001566 \\ \mathrm{C} & -1.100992 & 1.219747 & 0.001085 \\ \mathrm{C} & -0.230494 & 0.120908 & -0.000625 \\ \mathrm{H} & -0.077007 & -2.028559 & -0.002676 \\ \mathrm{H} & -2.533667 & -2.385214 & -0.001516 \\ \mathrm{H} & -4.070628 & -0.435157 & 0.000975 \\ \mathrm{H} & -3.150322 & 1.872858 & 0.002771 \\ \mathrm{H} & -0.679953 & 2.219305 & 0.002106 \\ \mathrm{C} & 1.235111 & 0.392586 & -0.000951 \\ \mathrm{O} & 1.729938 & 1.504701 & -0.002863 \\ \mathrm{O} & 1.971082 & -0.739483 & 0.001338 \\ \mathrm{C} & 3.396168 & -0.551063 & 0.001730 \\ \mathrm{H} & 3.825634 & -1.553081 & 0.009790 \\ \mathrm{H} & 3.716104 & -0.009610 & -0.892220 \\ \mathrm{H} & 3.713757 & 0.003787 & 0.888157\end{array}$

4

Zero-point correction $=0.718741$

Thermal correction to Energy= 0.771482

Thermal correction to Enthalpy $=0.772426$

Thermal correction to Gibbs Free Energy= 0.621356

Sum of electronic and zero-point Energies $=-2220.368896$ 
Sum of electronic and thermal Energies $=-2220.316156$

Sum of electronic and thermal Enthalpies $=-2220.315212$

Sum of electronic and thermal Free Energies= -2220.466282

Cartesian coordinates

\begin{tabular}{|c|c|c|c|}
\hline $\mathrm{C}$ & 1.114255 & -3.662963 & -0.382484 \\
\hline $\mathrm{C}$ & 2.323439 & -3.781586 & 0.289165 \\
\hline $\mathrm{C}$ & 3.157180 & -2.666673 & 0.346929 \\
\hline $\mathrm{C}$ & 2.768333 & -1.488494 & -0.280996 \\
\hline $\mathrm{C}$ & 1.546982 & -1.468979 & -0.954652 \\
\hline $\mathrm{N}$ & 0.719558 & -2.531489 & -0.991046 \\
\hline $\mathrm{H}$ & 4.107568 & -2.693653 & 0.870028 \\
\hline $\mathrm{H}$ & 2.591440 & -4.721233 & 0.760025 \\
\hline $\mathrm{H}$ & 3.409594 & -0.623089 & -0.216261 \\
\hline $\mathrm{C}$ & 1.686985 & 0.854259 & -2.030164 \\
\hline $\mathrm{C}$ & -0.192391 & -0.457456 & -2.247315 \\
\hline $\mathrm{C}$ & 0.755442 & 1.514320 & -2.850464 \\
\hline $\mathrm{C}$ & 2.928895 & 1.436224 & -1.761323 \\
\hline $\mathrm{C}$ & 1.020150 & 2.755706 & -3.425741 \\
\hline $\mathrm{C}$ & 3.193421 & 2.680982 & -2.335000 \\
\hline $\mathrm{H}$ & 3.689586 & 0.977112 & -1.144421 \\
\hline $\mathrm{C}$ & 2.260370 & 3.332862 & -3.154246 \\
\hline $\mathrm{H}$ & 0.294483 & 3.251230 & -4.061098 \\
\hline $\mathrm{H}$ & 4.153462 & 3.145413 & -2.134379 \\
\hline $\mathrm{H}$ & 2.506042 & 4.298702 & -3.585131 \\
\hline $\mathrm{N}$ & 1.060625 & -0.358724 & -1.675310 \\
\hline $\mathrm{N}$ & -0.365849 & 0.681265 & -2.953447 \\
\hline $\mathrm{C}$ & -1.545362 & 1.013354 & -3.757679 \\
\hline $\mathrm{H}$ & -1.188352 & 1.410298 & -4.714757 \\
\hline $\mathrm{H}$ & -2.062863 & 0.078410 & -3.970293 \\
\hline $\mathrm{C}$ & -2.487646 & 2.013819 & -3.077924 \\
\hline $\mathrm{H}$ & -2.796601 & 1.624847 & -2.101869 \\
\hline $\mathrm{H}$ & -1.949707 & 2.948437 & -2.880333 \\
\hline $\mathrm{C}$ & -3.718121 & 2.295917 & -3.942805 \\
\hline $\mathrm{H}$ & -4.300635 & 1.384066 & -4.113966 \\
\hline $\mathrm{H}$ & -4.375846 & 3.020749 & -3.453864 \\
\hline $\mathrm{H}$ & -3.439773 & 2.705041 & -4.921664 \\
\hline $\mathrm{H}$ & 0.427574 & -4.498984 & -0.442503 \\
\hline Mn & -1.190408 & -2.175489 & -1.855627 \\
\hline $\mathrm{C}$ & -1.863666 & -3.822859 & -1.422077 \\
\hline $\mathrm{O}$ & -2.299007 & -4.886471 & -1.275282 \\
\hline $\mathrm{C}$ & -0.723996 & -2.803853 & -3.477707 \\
\hline $\mathrm{O}$ & -0.456623 & -3.191840 & -4.541193 \\
\hline $\mathrm{C}$ & -2.844997 & -1.660391 & -2.329473 \\
\hline $\mathrm{O}$ & -3.940624 & -1.350850 & -2.556937 \\
\hline $\mathrm{C}$ & -3.431218 & -0.881239 & 1.636348 \\
\hline $\mathrm{C}$ & -4.165056 & -1.311024 & 2.737158 \\
\hline $\mathrm{C}$ & -3.990199 & -2.587254 & 3.295060 \\
\hline $\mathrm{C}$ & -3.018947 & -3.405009 & 2.697922 \\
\hline $\mathrm{C}$ & -2.265834 & -2.986652 & 1.604953 \\
\hline $\mathrm{C}$ & -2.449292 & -1.705860 & 1.020507 \\
\hline $\mathrm{H}$ & -3.596610 & 0.117340 & 1.235283 \\
\hline $\mathrm{H}$ & -4.901908 & -0.636042 & 3.171376 \\
\hline $\mathrm{H}$ & -2.835336 & -4.397000 & 3.109865 \\
\hline $\mathrm{H}$ & -1.493794 & -3.638838 & 1.210216 \\
\hline C & -4.825963 & -3.069713 & 4.457555 \\
\hline $\mathrm{H}$ & -5.180560 & -2.233600 & 5.074287 \\
\hline $\mathrm{H}$ & -5.720199 & -3.622145 & 4.128294 \\
\hline $\mathrm{H}$ & -4.259776 & -3.744873 & 5.111571 \\
\hline $\mathrm{N}$ & -1.678281 & -1.257760 & -0.030062 \\
\hline $\mathrm{H}$ & -1.942112 & -0.294250 & -0.218449 \\
\hline $\mathrm{H}$ & -2.757545 & 2.688576 & 1.252200 \\
\hline I & 6.411349 & 0.456995 & 0.623945 \\
\hline $\mathrm{C}$ & -3.775278 & 5.025767 & 1.526543 \\
\hline
\end{tabular}




$\begin{array}{lrrr}\mathrm{C} & -4.413336 & 3.733249 & 0.991740 \\ \mathrm{H} & -3.117280 & 4.809968 & 2.375356 \\ \mathrm{H} & -3.179447 & 5.511196 & 0.746203 \\ \mathrm{H} & -4.537086 & 5.737557 & 1.864567 \\ \mathrm{C} & -5.287261 & 4.028673 & -0.230060 \\ \mathrm{C} & -5.237078 & 3.045176 & 2.091642 \\ \mathrm{H} & -5.706350 & 3.100679 & -0.632007 \\ \mathrm{H} & -6.115358 & 4.697091 & 0.028471 \\ \mathrm{H} & -4.693961 & 4.505414 & -1.017557 \\ \mathrm{H} & -6.035923 & 3.697541 & 2.463377 \\ \mathrm{H} & -5.694849 & 2.127710 & 1.708712 \\ \mathrm{H} & -4.599959 & 2.776870 & 2.941750 \\ \mathrm{O} & -3.388872 & 2.842148 & 0.525040 \\ \mathrm{C} & 2.030147 & 1.029960 & 2.921496 \\ \mathrm{C} & 2.706966 & 0.071665 & 3.673588 \\ \mathrm{C} & 2.000765 & -0.747560 & 4.558500 \\ \mathrm{C} & 0.615524 & -0.612801 & 4.690195 \\ \mathrm{C} & -0.065466 & 0.345029 & 3.943699 \\ \mathrm{C} & 0.640376 & 1.172064 & 3.056938 \\ \mathrm{H} & 2.576759 & 1.661915 & 2.231235 \\ \mathrm{H} & 3.781378 & -0.034668 & 3.559093 \\ \mathrm{H} & 2.530344 & -1.496166 & 5.141703 \\ \mathrm{H} & 0.065258 & -1.258911 & 5.367874 \\ \mathrm{H} & -1.141357 & 0.452559 & 4.023204 \\ \mathrm{C} & -0.122680 & 2.187484 & 2.288053 \\ \mathrm{O} & -1.310681 & 2.432630 & 2.466871 \\ \mathrm{O} & 0.627498 & 2.826414 & 1.376360 \\ \mathrm{C} & -0.010087 & 3.881035 & 0.633849 \\ \mathrm{H} & 0.713888 & 4.176760 & -0.125437 \\ \mathrm{H} & -0.935016 & 3.534626 & 0.168770 \\ \mathrm{H} & -0.228522 & 4.727451 & 1.291933\end{array}$

5-ts

Zero-point correction $=0.719699$

Thermal correction to Energy $=0.770269$

Thermal correction to Enthalpy $=0.771213$

Thermal correction to Gibbs Free Energy $=0.629793$

Sum of electronic and zero-point Energies= -2220.330093

Sum of electronic and thermal Energies= -2220.279523

Sum of electronic and thermal Enthalpies $=-2220.278579$

Sum of electronic and thermal Free Energies= -2220.419999

Cartesian coordinates

$\begin{array}{llrr}\mathrm{C} & -1.239920 & -2.828237 & 1.292637 \\ \mathrm{C} & -2.426066 & -3.403497 & 0.859368 \\ \mathrm{C} & -3.434518 & -2.562441 & 0.392320 \\ \mathrm{C} & -3.223733 & -1.188314 & 0.371355 \\ \mathrm{C} & -2.001640 & -0.693284 & 0.829206 \\ \mathrm{~N} & -1.018756 & -1.499832 & 1.284415 \\ \mathrm{H} & -4.387613 & -2.950105 & 0.046874 \\ \mathrm{H} & -2.546766 & -4.480310 & 0.891384 \\ \mathrm{H} & -4.020081 & -0.546347 & 0.028059 \\ \mathrm{C} & -2.431431 & 1.809390 & 0.525070 \\ \mathrm{C} & -0.468885 & 1.035671 & 1.449754 \\ \mathrm{C} & -1.633741 & 2.902816 & 0.899853 \\ \mathrm{C} & -3.675402 & 2.016500 & -0.079584 \\ \mathrm{C} & -2.046262 & 4.221555 & 0.716736 \\ \mathrm{C} & -4.084567 & 3.336868 & -0.270213 \\ \mathrm{H} & -4.333393 & 1.220843 & -0.402021 \\ \mathrm{C} & -3.291187 & 4.423288 & 0.124303 \\ \mathrm{H} & -1.424505 & 5.057117 & 1.017029 \\ \mathrm{H} & -5.048513 & 3.513251 & -0.736297 \\ \mathrm{H} & -3.647232 & 5.436059 & -0.037400 \\ \mathrm{~N} & -1.683511 & 0.673774 & 0.898270\end{array}$




\begin{tabular}{|c|c|c|c|}
\hline $\mathrm{N}$ & -0.456341 & 2.385809 & 1.457367 \\
\hline $\mathrm{C}$ & 0.591346 & 3.250713 & 2.013454 \\
\hline $\mathrm{H}$ & 0.092576 & 3.976703 & 2.666409 \\
\hline $\mathrm{H}$ & 1.221187 & 2.631864 & 2.648596 \\
\hline $\mathrm{C}$ & 1.433613 & 3.971350 & 0.954846 \\
\hline $\mathrm{H}$ & 1.920449 & 3.249446 & 0.291527 \\
\hline $\mathrm{H}$ & 0.781336 & 4.577486 & 0.316211 \\
\hline $\mathrm{C}$ & 2.490098 & 4.865813 & 1.609410 \\
\hline $\mathrm{H}$ & 3.176036 & 4.282524 & 2.234680 \\
\hline $\mathrm{H}$ & 3.090759 & 5.370872 & 0.847215 \\
\hline $\mathrm{H}$ & 2.036701 & 5.638250 & 2.242919 \\
\hline $\mathrm{H}$ & -0.429908 & -3.445056 & 1.660779 \\
\hline Mn & 0.656724 & -0.536461 & 2.116780 \\
\hline $\mathrm{C}$ & 1.408243 & -2.107751 & 2.706136 \\
\hline $\mathrm{O}$ & 1.819356 & -3.065070 & 3.207296 \\
\hline $\mathrm{C}$ & -0.224807 & -0.399229 & 3.678815 \\
\hline $\mathrm{O}$ & -0.785471 & -0.296775 & 4.687585 \\
\hline $\mathrm{C}$ & 2.066573 & 0.365841 & 2.810820 \\
\hline $\mathrm{O}$ & 2.968071 & 0.890562 & 3.315212 \\
\hline $\mathrm{C}$ & 4.284388 & -0.161795 & 0.229998 \\
\hline $\mathrm{C}$ & 5.585325 & -0.648971 & 0.368955 \\
\hline $\mathrm{C}$ & 5.839651 & -2.012650 & 0.554882 \\
\hline $\mathrm{C}$ & 4.736111 & -2.878097 & 0.582965 \\
\hline $\mathrm{C}$ & 3.433960 & -2.400786 & 0.451427 \\
\hline $\mathrm{C}$ & 3.183280 & -1.028777 & 0.291403 \\
\hline $\mathrm{H}$ & 4.119529 & 0.894437 & 0.036429 \\
\hline $\mathrm{H}$ & 6.418407 & 0.049044 & 0.320385 \\
\hline $\mathrm{H}$ & 4.896896 & -3.946884 & 0.708035 \\
\hline $\mathrm{H}$ & 2.603784 & -3.097660 & 0.462322 \\
\hline $\mathrm{C}$ & 7.244872 & -2.535968 & 0.737433 \\
\hline $\mathrm{H}$ & 7.990147 & -1.805940 & 0.405756 \\
\hline $\mathrm{H}$ & 7.457811 & -2.763193 & 1.790512 \\
\hline $\mathrm{H}$ & 7.407878 & -3.460793 & 0.172367 \\
\hline $\mathrm{N}$ & 1.840638 & -0.538230 & 0.150330 \\
\hline $\mathrm{H}$ & 1.922873 & 0.476132 & 0.124352 \\
\hline $\mathrm{H}$ & 2.743404 & 1.640924 & -1.870535 \\
\hline I & -7.053241 & -0.501568 & -0.941434 \\
\hline $\mathrm{C}$ & 2.954125 & 3.075734 & -4.021292 \\
\hline $\mathrm{C}$ & 3.859631 & 3.005545 & -2.778578 \\
\hline $\mathrm{H}$ & 2.575702 & 2.078737 & -4.269049 \\
\hline $\mathrm{H}$ & 2.095949 & 3.729268 & -3.829900 \\
\hline $\mathrm{H}$ & 3.491498 & 3.463782 & -4.895179 \\
\hline $\mathrm{C}$ & 4.368326 & 4.402128 & -2.406360 \\
\hline $\mathrm{C}$ & 5.040895 & 2.054133 & -3.039158 \\
\hline $\mathrm{H}$ & 4.975946 & 4.354422 & -1.496277 \\
\hline $\mathrm{H}$ & 4.981896 & 4.830892 & -3.206433 \\
\hline $\mathrm{H}$ & 3.527580 & 5.078957 & -2.219533 \\
\hline $\mathrm{H}$ & 5.628381 & 2.366670 & -3.911275 \\
\hline $\mathrm{H}$ & 5.709236 & 2.026539 & -2.171759 \\
\hline $\mathrm{H}$ & 4.675414 & 1.038076 & -3.216616 \\
\hline $\mathrm{O}$ & 3.102655 & 2.546014 & -1.658367 \\
\hline $\mathrm{C}$ & 0.044092 & -2.762444 & -1.880745 \\
\hline $\mathrm{C}$ & -0.051613 & -4.078976 & -2.340509 \\
\hline $\mathrm{C}$ & 1.057654 & -4.706344 & -2.910453 \\
\hline $\mathrm{C}$ & 2.258636 & -4.002023 & -3.032717 \\
\hline $\mathrm{C}$ & 2.347915 & -2.685044 & -2.581789 \\
\hline $\mathrm{C}$ & 1.247505 & -2.055551 & -1.983121 \\
\hline $\mathrm{H}$ & -0.823793 & -2.265990 & -1.465189 \\
\hline $\mathrm{H}$ & -0.998110 & -4.608260 & -2.264390 \\
\hline $\mathrm{H}$ & 0.983497 & -5.730103 & -3.268572 \\
\hline $\mathrm{H}$ & 3.124186 & -4.476070 & -3.488980 \\
\hline $\mathrm{H}$ & 3.263364 & -2.116677 & -2.697958 \\
\hline $\mathrm{C}$ & 1.384909 & -0.591720 & -1.555166 \\
\hline
\end{tabular}




$\begin{array}{lrrr}\mathrm{O} & 2.253763 & 0.080640 & -2.202529 \\ \mathrm{O} & 0.087493 & 0.011418 & -1.390079 \\ \mathrm{C} & -0.390518 & 0.632131 & -2.578547 \\ \mathrm{H} & -1.398284 & 0.993355 & -2.357018 \\ \mathrm{H} & 0.238189 & 1.477309 & -2.876947 \\ \mathrm{H} & -0.451036 & -0.075360 & -3.417121\end{array}$

6

Zero-point correction $=0.719926$

Thermal correction to Energy $=0.771176$

Thermal correction to Enthalpy $=0.772120$

Thermal correction to Gibbs Free Energy= 0.628952

Sum of electronic and zero-point Energies $=-2220.329826$

Sum of electronic and thermal Energies $=-2220.278575$

Sum of electronic and thermal Enthalpies= -2220.277631

Sum of electronic and thermal Free Energies= -2220.420799

Cartesian coordinates

\begin{tabular}{|c|c|c|c|}
\hline $\mathrm{C}$ & -1.231883 & -2.820617 & 1.314219 \\
\hline $\mathrm{C}$ & -2.416138 & -3.402765 & 0.883348 \\
\hline $\mathrm{C}$ & -3.425181 & -2.568390 & 0.405765 \\
\hline $\mathrm{C}$ & -3.218232 & -1.193877 & 0.373455 \\
\hline $\mathrm{C}$ & -1.998635 & -0.691339 & 0.831753 \\
\hline $\mathrm{N}$ & -1.014618 & -1.491673 & 1.294363 \\
\hline $\mathrm{H}$ & -4.375510 & -2.961857 & 0.060433 \\
\hline $\mathrm{H}$ & -2.533863 & -4.479625 & 0.925733 \\
\hline $\mathrm{H}$ & -4.014037 & -0.557221 & 0.019411 \\
\hline $\mathrm{C}$ & -2.437968 & 1.808654 & 0.515653 \\
\hline $\mathrm{C}$ & -0.467674 & 1.045844 & 1.433385 \\
\hline $\mathrm{C}$ & -1.641182 & 2.906395 & 0.878508 \\
\hline $\mathrm{C}$ & -3.688673 & 2.009210 & -0.078174 \\
\hline $\mathrm{C}$ & -2.058729 & 4.222983 & 0.692356 \\
\hline $\mathrm{C}$ & -4.103203 & 3.327304 & -0.272563 \\
\hline $\mathrm{H}$ & -4.347981 & 1.209968 & -0.388554 \\
\hline $\mathrm{C}$ & -3.308661 & 4.418104 & 0.109320 \\
\hline $\mathrm{H}$ & -1.437106 & 5.061727 & 0.982894 \\
\hline $\mathrm{H}$ & -5.071767 & 3.498324 & -0.730055 \\
\hline $\mathrm{H}$ & -3.669923 & 5.428965 & -0.054967 \\
\hline $\mathrm{N}$ & -1.684551 & 0.677149 & 0.890806 \\
\hline $\mathrm{N}$ & -0.458645 & 2.396032 & 1.433308 \\
\hline $\mathrm{C}$ & 0.588771 & 3.268061 & 1.977937 \\
\hline $\mathrm{H}$ & 0.089972 & 3.997800 & 2.627698 \\
\hline $\mathrm{H}$ & 1.223583 & 2.656309 & 2.615065 \\
\hline $\mathrm{C}$ & 1.424730 & 3.983273 & 0.910638 \\
\hline $\mathrm{H}$ & 1.913266 & 3.257081 & 0.253245 \\
\hline $\mathrm{H}$ & 0.767588 & 4.580673 & 0.267699 \\
\hline $\mathrm{C}$ & 2.479081 & 4.887755 & 1.554715 \\
\hline $\mathrm{H}$ & 3.171118 & 4.311925 & 2.179190 \\
\hline $\mathrm{H}$ & 3.072481 & 5.392584 & 0.785790 \\
\hline $\mathrm{H}$ & 2.023227 & 5.661383 & 2.185755 \\
\hline $\mathrm{H}$ & -0.420907 & -3.432121 & 1.688044 \\
\hline $\mathrm{Mn}$ & 0.661863 & -0.517868 & 2.110826 \\
\hline $\mathrm{C}$ & 1.417555 & -2.084064 & 2.709874 \\
\hline $\mathrm{O}$ & 1.831089 & -3.037405 & 3.216390 \\
\hline $\mathrm{C}$ & -0.214556 & -0.367427 & 3.673975 \\
\hline $\mathrm{O}$ & -0.773823 & -0.255404 & 4.681695 \\
\hline $\mathrm{C}$ & 2.071336 & 0.392028 & 2.795837 \\
\hline $\mathrm{O}$ & 2.971873 & 0.920980 & 3.298691 \\
\hline $\mathrm{C}$ & 4.292136 & -0.167686 & 0.229602 \\
\hline $\mathrm{C}$ & 5.590280 & -0.658855 & 0.378848 \\
\hline $\mathrm{C}$ & 5.837819 & -2.023776 & 0.567711 \\
\hline $\mathrm{C}$ & 4.731323 & -2.885155 & 0.590233 \\
\hline $\mathrm{C}$ & 3.431796 & -2.402806 & 0.449127 \\
\hline $\mathrm{C}$ & 3.188801 & -1.030589 & 0.284542 \\
\hline
\end{tabular}




\begin{tabular}{|c|c|c|c|}
\hline $\mathrm{H}$ & 4.131798 & 0.888278 & 0.030774 \\
\hline $\mathrm{H}$ & 6.426935 & 0.035036 & 0.334762 \\
\hline $\mathrm{H}$ & 4.886351 & -3.954019 & 0.718297 \\
\hline $\mathrm{H}$ & 2.598206 & -3.095641 & 0.457245 \\
\hline $\mathrm{C}$ & 7.240389 & -2.551433 & 0.761352 \\
\hline $\mathrm{H}$ & 7.989101 & -1.839695 & 0.399668 \\
\hline $\mathrm{H}$ & 7.456981 & -2.743490 & 1.820504 \\
\hline $\mathrm{H}$ & 7.391911 & -3.497370 & 0.228118 \\
\hline $\mathrm{N}$ & 1.847200 & -0.532087 & 0.134619 \\
\hline $\mathrm{H}$ & 1.936749 & 0.482063 & 0.117946 \\
\hline $\mathrm{H}$ & 2.753320 & 1.612533 & -1.872067 \\
\hline I & -7.061234 & -0.512968 & -0.933637 \\
\hline $\mathrm{C}$ & 2.953616 & 3.081782 & -4.005108 \\
\hline $\mathrm{C}$ & 3.867607 & 2.988789 & -2.769797 \\
\hline $\mathrm{H}$ & 2.565170 & 2.090950 & -4.260970 \\
\hline $\mathrm{H}$ & 2.102062 & 3.739667 & -3.799614 \\
\hline $\mathrm{H}$ & 3.488065 & 3.475411 & -4.878455 \\
\hline $\mathrm{C}$ & 4.385966 & 4.377356 & -2.381451 \\
\hline $\mathrm{C}$ & 5.041245 & 2.035344 & -3.051897 \\
\hline $\mathrm{H}$ & 5.003355 & 4.313563 & -1.478650 \\
\hline $\mathrm{H}$ & 4.993464 & 4.816072 & -3.181543 \\
\hline $\mathrm{H}$ & 3.550867 & 5.055006 & -2.176455 \\
\hline $\mathrm{H}$ & 5.627338 & 2.358157 & -3.921543 \\
\hline $\mathrm{H}$ & 5.714531 & 1.988776 & -2.188810 \\
\hline $\mathrm{H}$ & 4.669337 & 1.024343 & -3.244516 \\
\hline $\mathrm{O}$ & 3.116629 & 2.516934 & -1.651539 \\
\hline $\mathrm{C}$ & 0.046297 & -2.769033 & -1.859973 \\
\hline $\mathrm{C}$ & -0.054429 & -4.084422 & -2.322355 \\
\hline $\mathrm{C}$ & 1.050860 & -4.712433 & -2.900169 \\
\hline $\mathrm{C}$ & 2.251043 & -4.008167 & -3.029555 \\
\hline $\mathrm{C}$ & 2.344415 & -2.692568 & -2.576690 \\
\hline $\mathrm{C}$ & 1.249940 & -2.063124 & -1.968299 \\
\hline $\mathrm{H}$ & -0.818925 & -2.272591 & -1.438439 \\
\hline $\mathrm{H}$ & -1.001070 & -4.613004 & -2.241141 \\
\hline $\mathrm{H}$ & 0.972902 & -5.735492 & -3.260390 \\
\hline $\mathrm{H}$ & 3.112792 & -4.482398 & -3.493105 \\
\hline $\mathrm{H}$ & 3.258984 & -2.124111 & -2.699162 \\
\hline $\mathrm{C}$ & 1.393406 & -0.597287 & -1.536006 \\
\hline $\mathrm{O}$ & 2.255691 & 0.070923 & -2.204159 \\
\hline $\mathrm{O}$ & 0.090632 & 0.007128 & -1.377623 \\
\hline $\mathrm{C}$ & -0.389107 & 0.611453 & -2.572570 \\
\hline $\mathrm{H}$ & -1.395899 & 0.977221 & -2.354300 \\
\hline $\mathrm{H}$ & 0.239351 & 1.452653 & -2.884680 \\
\hline $\mathrm{H}$ & -0.452401 & -0.106175 & -3.403337 \\
\hline
\end{tabular}

7-ts

Zero-point correction $=0.719092$

Thermal correction to Energy $=0.770039$

Thermal correction to Enthalpy $=0.770983$

Thermal correction to Gibbs Free Energy= 0.625125

Sum of electronic and zero-point Energies $=-2220.312545$

Sum of electronic and thermal Energies $=-2220.261598$

Sum of electronic and thermal Enthalpies $=-2220.260654$

Sum of electronic and thermal Free Energies= -2220.406513

Cartesian coordinates

$\begin{array}{llll}\mathrm{C} & 1.600911 & -3.026673 & 0.317380 \\ \mathrm{C} & 2.704177 & -3.370081 & 1.086057 \\ \mathrm{C} & 3.774072 & -2.479086 & 1.139857 \\ \mathrm{C} & 3.700094 & -1.276766 & 0.444288 \\ \mathrm{C} & 2.543270 & -0.999727 & -0.282960 \\ \mathrm{~N} & 1.507278 & -1.864527 & -0.357594 \\ \mathrm{H} & 4.672088 & -2.699656 & 1.707784 \\ \mathrm{H} & 2.720103 & -4.316318 & 1.614584\end{array}$




\begin{tabular}{|c|c|c|c|}
\hline $\mathrm{H}$ & 4.545272 & -0.605481 & 0.475106 \\
\hline $\mathrm{C}$ & 3.089946 & 1.374279 & -1.084966 \\
\hline $\mathrm{C}$ & 1.173004 & 0.289806 & -1.762504 \\
\hline $\mathrm{C}$ & 2.343134 & 2.225714 & -1.915493 \\
\hline $\mathrm{C}$ & 4.303480 & 1.802480 & -0.539275 \\
\hline $\mathrm{C}$ & 2.769676 & 3.512915 & -2.237329 \\
\hline $\mathrm{C}$ & 4.731268 & 3.090122 & -0.862922 \\
\hline $\mathrm{H}$ & 4.924213 & 1.196782 & 0.107316 \\
\hline $\mathrm{C}$ & 3.983206 & 3.933218 & -1.697649 \\
\hline $\mathrm{H}$ & 2.182872 & 4.161618 & -2.877919 \\
\hline $\mathrm{H}$ & 5.673777 & 3.436370 & -0.451342 \\
\hline $\mathrm{H}$ & 4.351808 & 4.928565 & -1.925365 \\
\hline $\mathrm{N}$ & 2.330110 & 0.186578 & -1.012189 \\
\hline $\mathrm{N}$ & 1.191888 & 1.525130 & -2.301057 \\
\hline $\mathrm{C}$ & 0.128131 & 2.137366 & -3.104431 \\
\hline $\mathrm{H}$ & 0.611060 & 2.721547 & -3.894461 \\
\hline $\mathrm{H}$ & -0.421476 & 1.335283 & -3.593012 \\
\hline $\mathrm{C}$ & -0.812206 & 3.019146 & -2.272010 \\
\hline $\mathrm{H}$ & -1.245783 & 2.426259 & -1.458597 \\
\hline $\mathrm{H}$ & -0.231062 & 3.815115 & -1.792168 \\
\hline $\mathrm{C}$ & -1.919050 & 3.630579 & -3.134744 \\
\hline $\mathrm{H}$ & -2.539239 & 2.856602 & -3.601492 \\
\hline $\mathrm{H}$ & -2.577565 & 4.259136 & -2.528143 \\
\hline $\mathrm{H}$ & -1.508257 & 4.255588 & -3.936814 \\
\hline $\mathrm{H}$ & 0.756840 & -3.699195 & 0.228293 \\
\hline Mn & -0.007922 & -1.362412 & -1.722651 \\
\hline $\mathrm{C}$ & -1.032795 & -2.861473 & -1.356830 \\
\hline $\mathrm{O}$ & -1.659697 & -3.812085 & -1.178522 \\
\hline $\mathrm{C}$ & 0.922423 & -2.183726 & -2.999536 \\
\hline $\mathrm{O}$ & 1.533120 & -2.717667 & -3.826341 \\
\hline $\mathrm{C}$ & -1.248033 & -0.863286 & -2.947018 \\
\hline $\mathrm{O}$ & -2.027736 & -0.631120 & -3.771487 \\
\hline $\mathrm{C}$ & -4.739826 & -0.400766 & -1.398411 \\
\hline $\mathrm{C}$ & -6.019109 & -0.910985 & -1.616732 \\
\hline $\mathrm{C}$ & -6.458100 & -2.073213 & -0.972140 \\
\hline $\mathrm{C}$ & -5.554984 & -2.712489 & -0.109875 \\
\hline $\mathrm{C}$ & -4.270069 & -2.221379 & 0.104349 \\
\hline $\mathrm{C}$ & -3.837590 & -1.043566 & -0.532495 \\
\hline $\mathrm{H}$ & -4.433317 & 0.513030 & -1.901371 \\
\hline $\mathrm{H}$ & -6.689176 & -0.387044 & -2.295033 \\
\hline $\mathrm{H}$ & -5.859084 & -3.624689 & 0.399310 \\
\hline $\mathrm{H}$ & -3.595377 & -2.752903 & 0.763414 \\
\hline $\mathrm{C}$ & -7.855765 & -2.608536 & -1.174557 \\
\hline $\mathrm{H}$ & -7.867449 & -3.703771 & -1.206111 \\
\hline $\mathrm{H}$ & -8.526037 & -2.305394 & -0.359040 \\
\hline $\mathrm{H}$ & -8.295878 & -2.242514 & -2.107801 \\
\hline $\mathrm{N}$ & -2.517641 & -0.543231 & -0.372776 \\
\hline $\mathrm{H}$ & -2.435006 & 0.263936 & -0.987499 \\
\hline $\mathrm{H}$ & -3.210687 & 2.346513 & 2.264736 \\
\hline I & 7.471928 & -0.175656 & 1.555029 \\
\hline $\mathrm{C}$ & -5.521614 & 3.122593 & 1.373338 \\
\hline $\mathrm{C}$ & -4.448784 & 3.877559 & 2.179368 \\
\hline $\mathrm{H}$ & -5.042165 & 2.403675 & 0.702499 \\
\hline $\mathrm{H}$ & -6.181260 & 2.565007 & 2.047849 \\
\hline $\mathrm{H}$ & -6.141448 & 3.803447 & 0.776186 \\
\hline $\mathrm{C}$ & -5.104234 & 4.792142 & 3.220750 \\
\hline $\mathrm{C}$ & -3.553983 & 4.695876 & 1.230675 \\
\hline $\mathrm{H}$ & -4.339756 & 5.313462 & 3.807099 \\
\hline $\mathrm{H}$ & -5.748456 & 5.542643 & 2.748553 \\
\hline $\mathrm{H}$ & -5.713696 & 4.202348 & 3.914065 \\
\hline $\mathrm{H}$ & -4.124743 & 5.441558 & 0.662953 \\
\hline $\mathrm{H}$ & -2.776822 & 5.218195 & 1.799460 \\
\hline $\mathrm{H}$ & -3.059416 & 4.023636 & 0.522749 \\
\hline
\end{tabular}




$\begin{array}{rrrr}\mathrm{O} & -3.650188 & 2.956261 & 2.913623 \\ \mathrm{C} & -1.609431 & -1.900035 & 2.433998 \\ \mathrm{C} & -1.880472 & -2.714860 & 3.535259 \\ \mathrm{C} & -2.938586 & -2.403796 & 4.393568 \\ \mathrm{C} & -3.711558 & -1.268193 & 4.145243 \\ \mathrm{C} & -3.437636 & -0.455633 & 3.042835 \\ \mathrm{C} & -2.390050 & -0.766166 & 2.169682 \\ \mathrm{H} & -0.776795 & -2.127035 & 1.777566 \\ \mathrm{H} & -1.261499 & -3.587895 & 3.728979 \\ \mathrm{H} & -3.149616 & -3.034301 & 5.253661 \\ \mathrm{H} & -4.527925 & -1.007995 & 4.814464 \\ \mathrm{H} & -4.015634 & 0.443758 & 2.865227 \\ \mathrm{C} & -2.117112 & 0.152955 & 0.960835 \\ \mathrm{O} & -2.618912 & 1.344381 & 1.020188 \\ \mathrm{O} & -0.628762 & 0.139357 & 0.730825 \\ \mathrm{C} & 0.051778 & 1.025096 & 1.605467 \\ \mathrm{H} & 1.055697 & 1.192989 & 1.206156 \\ \mathrm{H} & -0.474711 & 1.982778 & 1.672531 \\ \mathrm{H} & 0.151909 & 0.605283 & 2.617968\end{array}$

$\mathbf{P}$

Zero-point correction $=0.237007$

Thermal correction to Energy $=0.250603$

Thermal correction to Enthalpy $=0.251547$

Thermal correction to Gibbs Free Energy= 0.195485

Sum of electronic and zero-point Energies $=-671.124400$

Sum of electronic and thermal Energies $=-671.110804$

Sum of electronic and thermal Enthalpies $=-671.109859$

Sum of electronic and thermal Free Energies $=-671.165922$

Cartesian coordinates

$\begin{array}{lrrr}\mathrm{C} & -2.165434 & 1.341941 & 0.298301 \\ \mathrm{C} & -3.555909 & 1.301969 & 0.299061 \\ \mathrm{C} & -4.249766 & 0.116093 & 0.024123 \\ \mathrm{C} & -3.488800 & -1.024963 & -0.258943 \\ \mathrm{C} & -2.094913 & -1.008083 & -0.264266 \\ \mathrm{C} & -1.417360 & 0.187259 & 0.019960 \\ \mathrm{H} & -1.651730 & 2.276725 & 0.512673 \\ \mathrm{H} & -4.110346 & 2.211409 & 0.516831 \\ \mathrm{H} & -3.997079 & -1.960094 & -0.481815 \\ \mathrm{H} & -1.529374 & -1.903039 & -0.479236 \\ \mathrm{C} & -5.757680 & 0.062008 & 0.062656 \\ \mathrm{H} & -6.152527 & -0.659866 & -0.659407 \\ \mathrm{H} & -6.122230 & -0.241221 & 1.052805 \\ \mathrm{H} & -6.201095 & 1.037514 & -0.160152 \\ \mathrm{~N} & -0.012233 & 0.310105 & 0.027777 \\ \mathrm{H} & 0.327303 & 1.236594 & 0.244153 \\ \mathrm{C} & 2.753137 & 1.109396 & -0.433425 \\ \mathrm{C} & 4.094190 & 1.489209 & -0.377105 \\ \mathrm{C} & 5.059672 & 0.586043 & 0.070324 \\ \mathrm{C} & 4.681802 & -0.704864 & 0.448359 \\ \mathrm{C} & 3.344812 & -1.088658 & 0.383520 \\ \mathrm{C} & 2.365180 & -0.181135 & -0.043335 \\ \mathrm{H} & 2.021574 & 1.814834 & -0.817335 \\ \mathrm{H} & 4.384573 & 2.487399 & -0.691214 \\ \mathrm{H} & 6.103160 & 0.883838 & 0.116422 \\ \mathrm{H} & 5.430559 & -1.412850 & 0.791319 \\ \mathrm{H} & 3.037354 & -2.091135 & 0.660808 \\ \mathrm{C} & 0.943172 & -0.671577 & -0.088752 \\ \mathrm{O} & 0.684109 & -1.867333 & -0.205395\end{array}$

8

Zero-point correction $=0.172417$

Thermal correction to Energy $=0.182709$ 
Thermal correction to Enthalpy $=0.183653$

Thermal correction to Gibbs Free Energy= 0.137226

Sum of electronic and zero-point Energies $=-348.703164$

Sum of electronic and thermal Energies $=-348.692872$

Sum of electronic and thermal Enthalpies $=-348.691928$

Sum of electronic and thermal Free Energies= -348.738355

Cartesian coordinates

$\begin{array}{lrrr}\text { H } & 0.885907 & 0.152749 & -0.592057 \\ \mathrm{C} & -1.188047 & 1.467867 & 0.307561 \\ \mathrm{C} & -1.037553 & -0.036177 & -0.025774 \\ \mathrm{H} & -0.233269 & 1.851312 & 0.680681 \\ \mathrm{H} & -1.440472 & 2.027030 & -0.601763 \\ \mathrm{H} & -1.964792 & 1.667305 & 1.059418 \\ \mathrm{C} & -2.363413 & -0.578688 & -0.586106 \\ \mathrm{C} & -0.650691 & -0.803007 & 1.260693 \\ \mathrm{H} & -2.251050 & -1.636109 & -0.852123 \\ \mathrm{H} & -3.191314 & -0.489442 & 0.130066 \\ \mathrm{H} & -2.639384 & -0.036238 & -1.498338 \\ \mathrm{H} & -1.364533 & -0.657948 & 2.084038 \\ \mathrm{H} & -0.584260 & -1.877596 & 1.050532 \\ \mathrm{H} & 0.340207 & -0.457678 & 1.571949 \\ \mathrm{O} & -0.058322 & -0.218989 & -1.006889 \\ \mathrm{O} & 2.024748 & 0.599254 & 0.032845 \\ \mathrm{C} & 3.011885 & -0.325907 & -0.094780 \\ \mathrm{H} & 3.645788 & -0.228143 & -1.022536 \\ \mathrm{H} & 3.765060 & -0.307905 & 0.737552 \\ \mathrm{H} & 2.667621 & -1.393974 & -0.124631\end{array}$

9-ts

Zero-point correction $=0.170320$

Thermal correction to Energy $=0.180221$

Thermal correction to Enthalpy $=0.181166$

Thermal correction to Gibbs Free Energy= 0.135852

Sum of electronic and zero-point Energies $=-348.704936$

Sum of electronic and thermal Energies $=-348.695034$

Sum of electronic and thermal Enthalpies $=-348.694090$

Sum of electronic and thermal Free Energies= -348.739404

Cartesian coordinates

$\begin{array}{lrrr}\text { H } & 1.055129 & 0.252253 & -0.475103 \\ \mathrm{C} & -1.232781 & 1.464657 & 0.294642 \\ \mathrm{C} & -1.021518 & -0.038673 & -0.037745 \\ \mathrm{H} & -0.312221 & 1.876218 & 0.722114 \\ \mathrm{H} & -1.443886 & 2.018482 & -0.628682 \\ \mathrm{H} & -2.055555 & 1.650052 & 1.001299 \\ \mathrm{C} & -2.328273 & -0.604650 & -0.638902 \\ \mathrm{C} & -0.697479 & -0.793529 & 1.280620 \\ \mathrm{H} & -2.192942 & -1.664609 & -0.884328 \\ \mathrm{H} & -3.193289 & -0.513875 & 0.034058 \\ \mathrm{H} & -2.564287 & -0.080352 & -1.572961 \\ \mathrm{H} & -1.467143 & -0.680147 & 2.058866 \\ \mathrm{H} & -0.578074 & -1.864280 & 1.072722 \\ \mathrm{H} & 0.256960 & -0.421933 & 1.667729 \\ \mathrm{O} & -0.006004 & -0.201372 & -0.957599 \\ \mathrm{O} & 2.048610 & 0.633986 & 0.029114 \\ \mathrm{C} & 2.988630 & -0.359544 & -0.094722 \\ \mathrm{H} & 3.581700 & -0.325601 & -1.044343 \\ \mathrm{H} & 3.752912 & -0.326767 & 0.714062 \\ \mathrm{H} & 2.568374 & -1.389926 & -0.060905\end{array}$

10

Zero-point correction $=0.172438$

Thermal correction to Energy $=0.182682$

Thermal correction to Enthalpy $=0.183626$ 
Thermal correction to Gibbs Free Energy $=0.137273$

Sum of electronic and zero-point Energies $=-348.702872$

Sum of electronic and thermal Energies $=-348.692628$

Sum of electronic and thermal Enthalpies $=-348.691684$

Sum of electronic and thermal Free Energies $=-348.738037$

Cartesian coordinates

$\begin{array}{lrrr}\mathrm{H} & 1.159954 & 0.365828 & -0.435075 \\ \mathrm{C} & -1.384493 & 1.452327 & 0.238157 \\ \mathrm{C} & -1.022493 & -0.035901 & -0.041189 \\ \mathrm{H} & -0.517801 & 1.965728 & 0.670274 \\ \mathrm{H} & -1.622812 & 1.952774 & -0.708813 \\ \mathrm{H} & -2.237316 & 1.586348 & 0.921428 \\ \mathrm{C} & -2.272167 & -0.741111 & -0.628636 \\ \mathrm{C} & -0.660170 & -0.711233 & 1.313181 \\ \mathrm{H} & -2.038895 & -1.793384 & -0.832471 \\ \mathrm{H} & -3.152571 & -0.705269 & 0.030311 \\ \mathrm{H} & -2.540241 & -0.275911 & -1.585237 \\ \mathrm{H} & -1.454204 & -0.646763 & 2.073199 \\ \mathrm{H} & -0.436919 & -1.772318 & 1.145001 \\ \mathrm{H} & 0.245889 & -0.242379 & 1.711427 \\ \mathrm{O} & 0.009957 & -0.132388 & -0.938141 \\ \mathrm{O} & 2.125987 & 0.687235 & -0.000971 \\ \mathrm{C} & 2.969453 & -0.403773 & -0.078370 \\ \mathrm{H} & 3.551132 & -0.467175 & -1.029564 \\ \mathrm{H} & 3.732375 & -0.392753 & 0.726900 \\ \mathrm{H} & 2.443066 & -1.375366 & 0.006655\end{array}$

11

Zero-point correction $=0.448163$

Thermal correction to Energy $=0.483678$

Thermal correction to Enthalpy $=0.484622$

Thermal correction to Gibbs Free Energy $=0.373737$

Sum of electronic and zero-point Energies $=-1660.496036$

Sum of electronic and thermal Energies $=-1660.460521$

Sum of electronic and thermal Enthalpies $=-1660.459577$

Sum of electronic and thermal Free Energies= -1660.570462

Cartesian coordinates

$\begin{array}{lrrr}\mathrm{C} & 0.506117 & -2.698473 & -1.910035 \\ \mathrm{C} & 1.771524 & -3.254337 & -1.781158 \\ \mathrm{C} & 2.787145 & -2.464138 & -1.247013 \\ \mathrm{C} & 2.516135 & -1.147022 & -0.887667 \\ \mathrm{C} & 1.225265 & -0.660327 & -1.078769 \\ \mathrm{~N} & 0.223293 & -1.429138 & -1.559940 \\ \mathrm{H} & 3.789821 & -2.850247 & -1.094181 \\ \mathrm{H} & 1.943102 & -4.281717 & -2.081013 \\ \mathrm{H} & 3.306843 & -0.558571 & -0.444453 \\ \mathrm{C} & 1.582674 & 1.792778 & -0.402647 \\ \mathrm{C} & -0.498262 & 0.996136 & -0.975115 \\ \mathrm{C} & 0.658226 & 2.849381 & -0.377420 \\ \mathrm{C} & 2.925027 & 2.017387 & -0.084178 \\ \mathrm{C} & 1.023911 & 4.153360 & -0.047237 \\ \mathrm{C} & 3.290799 & 3.321587 & 0.247770 \\ \mathrm{H} & 3.681078 & 1.243369 & -0.060849 \\ \mathrm{C} & 2.363187 & 4.374181 & 0.264288 \\ \mathrm{H} & 0.299845 & 4.960327 & -0.031257 \\ \mathrm{H} & 4.327277 & 3.514115 & 0.504566 \\ \mathrm{H} & 2.691430 & 5.374234 & 0.529079 \\ \mathrm{~N} & 0.825475 & 0.660349 & -0.780967 \\ \mathrm{~N} & -0.588877 & 2.317603 & -0.736835 \\ \mathrm{C} & -1.803572 & 3.137120 & -0.821325 \\ \mathrm{H} & -1.501998 & 4.112448 & -1.214173 \\ \mathrm{H} & -2.460280 & 2.686256 & -1.564624 \\ \mathrm{C} & -2.515981 & 3.296419 & 0.526761 \\ & & & \end{array}$




$\begin{array}{lrrr}\mathrm{H} & -2.823375 & 2.308372 & 0.888006 \\ \mathrm{H} & -1.808282 & 3.696065 & 1.262626 \\ \mathrm{C} & -3.734353 & 4.215974 & 0.416143 \\ \mathrm{H} & -4.468712 & 3.823470 & -0.295608 \\ \mathrm{H} & -4.233068 & 4.316657 & 1.384722 \\ \mathrm{H} & -3.449467 & 5.220980 & 0.084519 \\ \mathrm{H} & -0.317099 & -3.282265 & -2.302971 \\ \mathrm{Mn} & -1.688282 & -0.566586 & -1.515840 \\ \mathrm{C} & -2.564241 & -2.127599 & -1.977672 \\ \mathrm{O} & -3.139285 & -3.073452 & -2.301539 \\ \mathrm{C} & -1.560825 & -0.067728 & -3.238992 \\ \mathrm{O} & -1.450265 & 0.260207 & -4.340610 \\ \mathrm{C} & -3.288099 & 0.283957 & -1.346794 \\ \mathrm{O} & -4.319147 & 0.805104 & -1.309289 \\ \mathrm{I} & 5.919861 & -0.662875 & 1.019990 \\ \mathrm{C} & -3.820385 & -0.628313 & 3.366371 \\ \mathrm{C} & -5.167695 & -0.549702 & 3.708937 \\ \mathrm{C} & -6.146465 & -0.985806 & 2.811662 \\ \mathrm{C} & -5.777541 & -1.499081 & 1.566188 \\ \mathrm{C} & -4.432493 & -1.562957 & 1.209645 \\ \mathrm{C} & -3.446526 & -1.125605 & 2.106417 \\ \mathrm{H} & -3.057928 & -0.300326 & 4.063783 \\ \mathrm{H} & -5.454659 & -0.153941 & 4.678293 \\ \mathrm{H} & -7.195927 & -0.930615 & 3.085649 \\ \mathrm{H} & -6.535832 & -1.851943 & 0.874125 \\ \mathrm{H} & -4.143536 & -1.979319 & 0.252506 \\ \mathrm{C} & -2.015272 & -1.214245 & 1.728279 \\ \mathrm{O} & -1.565633 & -1.195350 & 0.577592 \\ \mathrm{O} & -1.217998 & -1.340418 & 2.783170 \\ \mathrm{C} & 0.208177 & -1.433683 & 2.558615 \\ \mathrm{H} & 0.648659 & -1.517705 & 3.550979 \\ \mathrm{H} & 0.440340 & -2.321544 & 1.967738 \\ \mathrm{H} & 0.576694 & -0.537088 & 2.058368\end{array}$

12

Zero-point correction $=0.442727$

Thermal correction to Energy $=0.479363$

Thermal correction to Enthalpy $=0.480307$

Thermal correction to Gibbs Free Energy= 0.370771

Sum of electronic and zero-point Energies $=-1660.347416$

Sum of electronic and thermal Energies $=-1660.310780$

Sum of electronic and thermal Enthalpies $=-1660.309836$

Sum of electronic and thermal Free Energies $=-1660.419372$

Cartesian coordinates

$\begin{array}{lrrr}\mathrm{C} & -1.546315 & 4.098371 & -1.134965 \\ \mathrm{C} & -2.791549 & 4.344417 & -0.557027 \\ \mathrm{C} & -3.585583 & 3.254670 & -0.199232 \\ \mathrm{C} & -3.116933 & 1.964301 & -0.442943 \\ \mathrm{C} & -1.864253 & 1.837095 & -1.047908 \\ \mathrm{~N} & -1.088424 & 2.863795 & -1.385867 \\ \mathrm{H} & -4.548729 & 3.399389 & 0.279523 \\ \mathrm{H} & -3.119763 & 5.363502 & -0.381917 \\ \mathrm{H} & -3.674409 & 1.085079 & -0.140497 \\ \mathrm{C} & -2.094692 & -0.331726 & -2.203691 \\ \mathrm{C} & -0.293515 & -0.125978 & -0.810563 \\ \mathrm{C} & -1.421035 & -1.553002 & -2.175181 \\ \mathrm{C} & -3.223412 & -0.133328 & -3.001932 \\ \mathrm{C} & -1.836794 & -2.634539 & -2.957092 \\ \mathrm{C} & -3.643963 & -1.211640 & -3.774684 \\ \mathrm{H} & -3.749320 & 0.813756 & -3.023418 \\ \mathrm{C} & -2.960147 & -2.441337 & -3.755244 \\ \mathrm{H} & -1.313225 & -3.583041 & -2.948261 \\ \mathrm{H} & -4.518395 & -1.099392 & -4.407712\end{array}$




$\begin{array}{lrrr}\mathrm{H} & -3.316407 & -3.258063 & -4.374986 \\ \mathrm{~N} & -1.378172 & 0.520246 & -1.353980 \\ \mathrm{~N} & -0.349012 & -1.397135 & -1.293438 \\ \mathrm{C} & 0.506033 & -2.546007 & -0.961967 \\ \mathrm{H} & 0.838416 & -2.986800 & -1.907857 \\ \mathrm{H} & 1.397760 & -2.175112 & -0.463276 \\ \mathrm{C} & -0.198861 & -3.590872 & -0.086601 \\ \mathrm{H} & -0.557221 & -3.115597 & 0.831288 \\ \mathrm{H} & -1.090230 & -3.964934 & -0.599581 \\ \mathrm{C} & 0.736528 & -4.757137 & 0.241756 \\ \mathrm{H} & 1.624986 & -4.421638 & 0.789219 \\ \mathrm{H} & 0.224253 & -5.492934 & 0.868465 \\ \mathrm{H} & 1.078446 & -5.271648 & -0.664310 \\ \mathrm{H} & -0.887467 & 4.917683 & -1.412374 \\ \mathrm{Mn} & 1.224572 & 0.780178 & 0.428741 \\ \mathrm{O} & 2.359438 & 1.740604 & 1.429296 \\ \mathrm{C} & 2.785152 & 1.587882 & 2.766644 \\ \mathrm{H} & 3.882779 & 1.573082 & 2.778793 \\ \mathrm{H} & 2.458033 & 2.460663 & 3.346909 \\ \mathrm{H} & 2.407441 & 0.679711 & 3.251707 \\ \mathrm{C} & 2.513732 & -0.079318 & -1.034107 \\ \mathrm{C} & 3.952663 & -0.209755 & -0.597877 \\ \mathrm{O} & 2.212280 & -0.244041 & -2.189556 \\ \mathrm{C} & 4.925775 & 0.198524 & -1.528870 \\ \mathrm{C} & 4.355860 & -0.731539 & 0.638484 \\ \mathrm{C} & 6.278037 & 0.100996 & -1.213064 \\ \mathrm{H} & 4.610226 & 0.590423 & -2.489828 \\ \mathrm{C} & 5.712810 & -0.856457 & 0.935351 \\ \mathrm{H} & 3.626023 & -1.070840 & 1.362299 \\ \mathrm{C} & 6.674485 & -0.429375 & 0.017679 \\ \mathrm{H} & 7.022797 & 0.431784 & -1.930861 \\ \mathrm{H} & 6.016928 & -1.283627 & 1.886135 \\ \mathrm{H} & 7.729965 & -0.512166 & 0.259634 \\ \mathrm{C} & 1.483274 & 2.277980 & -0.810346 \\ \mathrm{O} & 1.904484 & 3.123640 & -1.447129 \\ \mathrm{C} & -0.262692 & 1.594970 & 1.410927 \\ \mathrm{O} & -0.846710 & 2.260633 & 2.125616 \\ \mathrm{O} & 1.154376 & -0.708341 & 1.568092 \\ \mathrm{O} & 1.132877 & -1.606127 & 2.277484 \\ & -2.916618 & -0.984960 & 2.515582\end{array}$

4n

Zero-point correction $=0.717908$

Thermal correction to Energy $=0.767497$

Thermal correction to Enthalpy $=0.768441$

Thermal correction to Gibbs Free Energy= 0.626960

Sum of electronic and zero-point Energies $=-2208.786925$

Sum of electronic and thermal Energies $=-2208.737335$

Sum of electronic and thermal Enthalpies $=-2208.736391$

Sum of electronic and thermal Free Energies= -2208.877873

Cartesian coordinates

$\begin{array}{lrrr}\mathrm{C} & -3.462429 & 2.184435 & 0.325368 \\ \mathrm{C} & -3.709517 & 2.698767 & 1.592376 \\ \mathrm{C} & -3.328921 & 1.938397 & 2.695962 \\ \mathrm{C} & -2.764040 & 0.682356 & 2.499885 \\ \mathrm{C} & -2.594854 & 0.227905 & 1.190268 \\ \mathrm{~N} & -2.901731 & 0.982215 & 0.118200 \\ \mathrm{H} & -3.465933 & 2.314390 & 3.704736 \\ \mathrm{H} & -4.165785 & 3.675942 & 1.701022 \\ \mathrm{H} & -2.432692 & 0.095900 & 3.342904 \\ \mathrm{C} & -1.901870 & -2.194374 & 1.643863 \\ \mathrm{C} & -1.832853 & -1.305744 & -0.479855 \\ \mathrm{C} & -1.465003 & -3.181202 & 0.743240\end{array}$




\begin{tabular}{|c|c|c|c|}
\hline $\mathrm{C}$ & -2.075727 & -2.502913 & 2.995273 \\
\hline $\mathrm{C}$ & -1.165549 & -4.478625 & 1.152341 \\
\hline $\mathrm{C}$ & -1.778304 & -3.803107 & 3.407353 \\
\hline $\mathrm{H}$ & -2.446133 & -1.786380 & 3.714574 \\
\hline $\mathrm{C}$ & -1.324931 & -4.776101 & 2.505371 \\
\hline $\mathrm{H}$ & -0.829127 & -5.229938 & 0.446693 \\
\hline $\mathrm{H}$ & -1.907083 & -4.062842 & 4.453435 \\
\hline $\mathrm{H}$ & -1.103762 & -5.777377 & 2.861450 \\
\hline $\mathrm{N}$ & -2.098823 & -1.045240 & 0.854398 \\
\hline $\mathrm{N}$ & -1.447693 & -2.598886 & -0.529483 \\
\hline $\mathrm{C}$ & -1.087519 & -3.337463 & -1.745630 \\
\hline $\mathrm{H}$ & -1.561523 & -4.322909 & -1.678898 \\
\hline $\mathrm{H}$ & -1.546398 & -2.816456 & -2.585684 \\
\hline $\mathrm{C}$ & 0.424923 & -3.479486 & -1.947429 \\
\hline $\mathrm{H}$ & 0.887819 & -2.487940 & -1.982488 \\
\hline $\mathrm{H}$ & 0.861972 & -3.992560 & -1.082398 \\
\hline $\mathrm{C}$ & 0.742637 & -4.254755 & -3.228076 \\
\hline $\mathrm{H}$ & 0.352404 & -3.739162 & -4.112228 \\
\hline $\mathrm{H}$ & 1.823835 & -4.359139 & -3.358173 \\
\hline $\mathrm{H}$ & 0.310437 & -5.262437 & -3.208920 \\
\hline $\mathrm{H}$ & -3.714555 & 2.749719 & -0.564234 \\
\hline Mn & -2.149167 & 0.239132 & -1.733334 \\
\hline $\mathrm{C}$ & -2.551455 & 1.754290 & -2.692360 \\
\hline $\mathrm{O}$ & -2.882806 & 2.632728 & -3.368789 \\
\hline $\mathrm{C}$ & -3.719751 & -0.489997 & -2.241111 \\
\hline $\mathrm{O}$ & -4.716028 & -0.976247 & -2.590708 \\
\hline $\mathrm{C}$ & -1.200539 & -0.356585 & -3.141798 \\
\hline $\mathrm{O}$ & -0.543520 & -0.672437 & -4.043706 \\
\hline $\mathrm{C}$ & 1.783138 & 2.107208 & -1.553433 \\
\hline $\mathrm{C}$ & 2.481706 & 3.292872 & -1.761431 \\
\hline $\mathrm{C}$ & 1.843836 & 4.542998 & -1.751545 \\
\hline $\mathrm{C}$ & 0.464076 & 4.541434 & -1.499682 \\
\hline $\mathrm{C}$ & -0.247440 & 3.365757 & -1.276747 \\
\hline $\mathrm{C}$ & 0.382834 & 2.095328 & -1.306949 \\
\hline $\mathrm{H}$ & 2.314626 & 1.157526 & -1.565434 \\
\hline $\mathrm{H}$ & 3.554059 & 3.243153 & -1.946278 \\
\hline $\mathrm{H}$ & -0.069424 & 5.490883 & -1.466514 \\
\hline $\mathrm{H}$ & -1.305489 & 3.423447 & -1.045067 \\
\hline $\mathrm{C}$ & 2.596902 & 5.821920 & -2.032544 \\
\hline $\mathrm{H}$ & 3.637684 & 5.762968 & -1.693786 \\
\hline $\mathrm{H}$ & 2.625190 & 6.060097 & -3.105555 \\
\hline $\mathrm{H}$ & 2.135869 & 6.680295 & -1.530396 \\
\hline $\mathrm{N}$ & -0.294640 & 0.924475 & -1.038476 \\
\hline $\mathrm{H}$ & 0.377422 & 0.162409 & -1.066543 \\
\hline $\mathrm{H}$ & 3.369410 & -0.845801 & -0.106250 \\
\hline $\mathrm{C}$ & 5.582129 & -2.162152 & -0.255321 \\
\hline $\mathrm{C}$ & 4.709836 & -1.560367 & -1.368553 \\
\hline $\mathrm{H}$ & 5.614570 & -1.496697 & 0.614449 \\
\hline $\mathrm{H}$ & 5.180788 & -3.129240 & 0.066299 \\
\hline $\mathrm{H}$ & 6.612309 & -2.317754 & -0.594676 \\
\hline $\mathrm{C}$ & 4.625423 & -2.512629 & -2.563908 \\
\hline $\mathrm{C}$ & 5.260393 & -0.194029 & -1.804807 \\
\hline $\mathrm{H}$ & 3.968710 & -2.098079 & -3.335191 \\
\hline $\mathrm{H}$ & 5.613625 & -2.676586 & -3.005973 \\
\hline $\mathrm{H}$ & 4.222964 & -3.483144 & -2.254840 \\
\hline $\mathrm{H}$ & 6.286747 & -0.277373 & -2.180144 \\
\hline $\mathrm{H}$ & 4.639780 & 0.233219 & -2.598586 \\
\hline $\mathrm{H}$ & 5.268020 & 0.508848 & -0.964065 \\
\hline $\mathrm{O}$ & 3.358602 & -1.413284 & -0.897530 \\
\hline $\mathrm{C}$ & 1.064713 & 0.952302 & 4.440453 \\
\hline $\mathrm{C}$ & 0.506324 & 2.068252 & 5.060957 \\
\hline $\mathrm{C}$ & 0.613389 & 3.328371 & 4.464806 \\
\hline $\mathrm{C}$ & 1.284902 & 3.474414 & 3.248360 \\
\hline
\end{tabular}




$\begin{array}{rrrr}\mathrm{C} & 1.856881 & 2.364827 & 2.629780 \\ \mathrm{C} & 1.743872 & 1.098422 & 3.221240 \\ \mathrm{H} & 0.986510 & -0.027703 & 4.897611 \\ \mathrm{H} & -0.008187 & 1.957256 & 6.011336 \\ \mathrm{H} & 0.173572 & 4.195930 & 4.949152 \\ \mathrm{H} & 1.362012 & 4.449937 & 2.778272 \\ \mathrm{H} & 2.374138 & 2.461312 & 1.681537 \\ \mathrm{C} & 2.364730 & -0.057608 & 2.521798 \\ \mathrm{O} & 3.149402 & 0.052987 & 1.590066 \\ \mathrm{O} & 1.970812 & -1.242526 & 3.018683 \\ \mathrm{C} & 2.565765 & -2.417637 & 2.434522 \\ \mathrm{H} & 1.997180 & -3.258261 & 2.832935 \\ \mathrm{H} & 2.501916 & -2.393436 & 1.345171 \\ \mathrm{H} & 3.613964 & -2.500915 & 2.735282\end{array}$

\section{7n-ts}

Zero-point correction $=0.718158$

Thermal correction to Energy $=0.765069$

Thermal correction to Enthalpy $=0.766013$

Thermal correction to Gibbs Free Energy= 0.633853

Sum of electronic and zero-point Energies $=-2208.728287$

Sum of electronic and thermal Energies $=-2208.681376$

Sum of electronic and thermal Enthalpies $=-2208.680432$

Sum of electronic and thermal Free Energies= -2208.812592

Cartesian coordinates

$\begin{array}{lrrr}\mathrm{C} & 2.170514 & -3.064596 & 1.635367 \\ \mathrm{C} & 2.982398 & -3.347624 & 2.725224 \\ \mathrm{C} & 4.051029 & -2.493976 & 2.988898 \\ \mathrm{C} & 4.255965 & -1.382863 & 2.177149 \\ \mathrm{C} & 3.367180 & -1.151375 & 1.124894 \\ \mathrm{~N} & 2.348050 & -1.990637 & 0.843907 \\ \mathrm{H} & 4.728502 & -2.688282 & 3.814084 \\ \mathrm{H} & 2.783863 & -4.222123 & 3.333799 \\ \mathrm{H} & 5.100056 & -0.735036 & 2.352957 \\ \mathrm{C} & 4.237402 & 1.112083 & 0.303937 \\ \mathrm{C} & 2.570065 & -0.011324 & -0.825427 \\ \mathrm{C} & 3.841707 & 1.867044 & -0.811983 \\ \mathrm{C} & 5.214802 & 1.602599 & 1.174150 \\ \mathrm{C} & 4.411819 & 3.102292 & -1.114184 \\ \mathrm{C} & 5.785992 & 2.839419 & 0.874134 \\ \mathrm{H} & 5.526827 & 1.079541 & 2.066438 \\ \mathrm{C} & 5.399485 & 3.576784 & -0.253891 \\ \mathrm{H} & 4.095542 & 3.676403 & -1.977573 \\ \mathrm{H} & 6.545889 & 3.237454 & 1.538910 \\ \mathrm{H} & 5.868036 & 4.534850 & -0.454489 \\ \mathrm{~N} & 3.439216 & -0.048973 & 0.255294 \\ \mathrm{~N} & 2.831976 & 1.146186 & -1.463224 \\ \mathrm{C} & 2.110478 & 1.679067 & -2.626696 \\ \mathrm{H} & 2.852737 & 2.142468 & -3.284696 \\ \mathrm{H} & 1.688646 & 0.834733 & -3.167772 \\ \mathrm{C} & 1.020531 & 2.686199 & -2.237412 \\ \mathrm{H} & 0.323918 & 2.217604 & -1.532993 \\ \mathrm{H} & 1.478488 & 3.524865 & -1.700232 \\ \mathrm{C} & 0.268764 & 3.205367 & -3.465543 \\ \mathrm{H} & -0.229316 & 2.392519 & -4.006275 \\ \mathrm{H} & -0.500369 & 3.923872 & -3.167574 \\ \mathrm{H} & 0.941224 & 3.709814 & -4.169523 \\ \mathrm{H} & 1.346602 & -3.717428 & 1.375751 \\ \mathrm{Mn} & 1.325639 & -1.608269 & -0.956068 \\ \mathrm{C} & 0.150774 & -3.028364 & -0.755753 \\ \mathrm{O} & -0.552488 & -3.937445 & -0.678857 \\ \mathrm{C} & 2.533721 & -2.596758 & -1.815282 \\ \mathrm{O} & 3.328267 & -3.232853 & -2.368582 \\ & & & \\ & & & \end{array}$




\begin{tabular}{|c|c|c|c|}
\hline $\mathrm{C}$ & 0.513121 & -1.218653 & -2.533654 \\
\hline $\mathrm{O}$ & 0.010801 & -1.051393 & -3.562322 \\
\hline $\mathrm{C}$ & -3.178720 & -0.381952 & -2.185120 \\
\hline $\mathrm{C}$ & -4.363841 & -0.859813 & -2.742775 \\
\hline $\mathrm{C}$ & -5.042214 & -1.950068 & -2.185442 \\
\hline $\mathrm{C}$ & -4.476791 & -2.550569 & -1.050997 \\
\hline $\mathrm{C}$ & -3.286612 & -2.091494 & -0.493328 \\
\hline $\mathrm{C}$ & -2.614935 & -0.987581 & -1.048744 \\
\hline $\mathrm{H}$ & -2.681248 & 0.475151 & -2.632695 \\
\hline $\mathrm{H}$ & -4.770841 & -0.368742 & -3.623851 \\
\hline $\mathrm{H}$ & -4.972273 & -3.406584 & -0.597759 \\
\hline $\mathrm{H}$ & -2.873493 & -2.592338 & 0.372691 \\
\hline $\mathrm{C}$ & -6.343432 & -2.449073 & -2.766667 \\
\hline $\mathrm{H}$ & -6.410536 & -3.541793 & -2.725834 \\
\hline $\mathrm{H}$ & -7.207846 & -2.054349 & -2.216512 \\
\hline $\mathrm{H}$ & -6.459332 & -2.145454 & -3.811951 \\
\hline $\mathrm{N}$ & -1.367543 & -0.527130 & -0.542717 \\
\hline $\mathrm{H}$ & -1.066029 & 0.224344 & -1.158957 \\
\hline $\mathrm{H}$ & -2.420189 & 2.662607 & 1.574333 \\
\hline $\mathrm{C}$ & -4.195132 & 3.675217 & -0.017853 \\
\hline $\mathrm{C}$ & -3.313910 & 4.339537 & 1.056125 \\
\hline $\mathrm{H}$ & -3.653848 & 2.842158 & -0.476815 \\
\hline $\mathrm{H}$ & -5.108787 & 3.273797 & 0.434608 \\
\hline $\mathrm{H}$ & -4.487211 & 4.381835 & -0.804714 \\
\hline $\mathrm{C}$ & -4.102037 & 5.415573 & 1.810876 \\
\hline $\mathrm{C}$ & -2.058510 & 4.949385 & 0.405908 \\
\hline $\mathrm{H}$ & -3.474878 & 5.878448 & 2.580601 \\
\hline $\mathrm{H}$ & -4.459118 & 6.202609 & 1.137171 \\
\hline $\mathrm{H}$ & -4.969152 & 4.969361 & 2.309301 \\
\hline $\mathrm{H}$ & -2.311501 & 5.715551 & -0.337315 \\
\hline $\mathrm{H}$ & -1.422202 & 5.408976 & 1.170437 \\
\hline $\mathrm{H}$ & -1.479065 & 4.162870 & -0.088265 \\
\hline $\mathrm{O}$ & -2.928839 & 3.379009 & 2.034423 \\
\hline $\mathrm{C}$ & -1.516588 & -1.665961 & 2.502388 \\
\hline $\mathrm{C}$ & -2.201985 & -2.330977 & 3.520735 \\
\hline $\mathrm{C}$ & -3.425866 & -1.836761 & 3.979101 \\
\hline $\mathrm{C}$ & -3.947709 & -0.670150 & 3.417550 \\
\hline $\mathrm{C}$ & -3.259314 & -0.007406 & 2.398608 \\
\hline $\mathrm{C}$ & -2.040186 & -0.501772 & 1.923124 \\
\hline $\mathrm{H}$ & -0.559300 & -2.039338 & 2.155730 \\
\hline $\mathrm{H}$ & -1.779290 & -3.231432 & 3.960596 \\
\hline $\mathrm{H}$ & -3.961535 & -2.351538 & 4.772554 \\
\hline $\mathrm{H}$ & -4.892635 & -0.269930 & 3.776001 \\
\hline $\mathrm{H}$ & -3.647730 & 0.915319 & 1.983291 \\
\hline $\mathrm{C}$ & -1.307586 & 0.252582 & 0.795816 \\
\hline $\mathrm{O}$ & -1.641756 & 1.488399 & 0.615052 \\
\hline $\mathrm{O}$ & 0.177438 & 0.069510 & 1.048458 \\
\hline $\mathrm{C}$ & 0.644139 & 0.929838 & 2.073178 \\
\hline $\mathrm{H}$ & 1.731418 & 1.012665 & 1.988465 \\
\hline $\mathrm{H}$ & 0.204627 & 1.927630 & 1.968101 \\
\hline $\mathrm{H}$ & 0.403980 & 0.542454 & 3.075030 \\
\hline
\end{tabular}

4'

Zero-point correction $=0.719195$

Thermal correction to Energy $=0.774538$

Thermal correction to Enthalpy $=0.775482$

Thermal correction to Gibbs Free Energy $=0.612974$

Sum of electronic and zero-point Energies $=-2382.595475$

Sum of electronic and thermal Energies $=-2382.540132$

Sum of electronic and thermal Enthalpies $=-2382.539188$

Sum of electronic and thermal Free Energies= -2382.701696

Cartesian coordinates

$\begin{array}{llll}\text { C } & 1.160421 & -3.322305 & 1.135830\end{array}$ 


\begin{tabular}{|c|c|c|c|}
\hline $\mathrm{C}$ & 2.200932 & -2.994718 & 1.996343 \\
\hline $\mathrm{C}$ & 2.824089 & -1.758853 & 1.834628 \\
\hline $\mathrm{C}$ & 2.418513 & -0.917402 & 0.803333 \\
\hline $\mathrm{C}$ & 1.390526 & -1.347571 & -0.037628 \\
\hline $\mathrm{N}$ & 0.745809 & -2.515563 & 0.145313 \\
\hline $\mathrm{H}$ & 3.619476 & -1.439537 & 2.500105 \\
\hline $\mathrm{H}$ & 2.492905 & -3.683397 & 2.780988 \\
\hline $\mathrm{H}$ & 2.878274 & 0.051234 & 0.688684 \\
\hline $\mathrm{C}$ & 1.452016 & 0.521272 & -1.788640 \\
\hline $\mathrm{C}$ & -0.183362 & -1.100130 & -1.823684 \\
\hline $\mathrm{C}$ & 0.599845 & 0.747297 & -2.883513 \\
\hline $\mathrm{C}$ & 2.559264 & 1.347081 & -1.575283 \\
\hline $\mathrm{C}$ & 0.802603 & 1.795743 & -3.778431 \\
\hline $\mathrm{C}$ & 2.764178 & 2.397699 & -2.472273 \\
\hline $\mathrm{H}$ & 3.261478 & 1.201382 & -0.766760 \\
\hline $\mathrm{C}$ & 1.901784 & 2.624106 & -3.554388 \\
\hline $\mathrm{H}$ & 0.136168 & 1.958818 & -4.617969 \\
\hline $\mathrm{H}$ & 3.617133 & 3.051427 & -2.319419 \\
\hline $\mathrm{H}$ & 2.093454 & 3.451696 & -4.230303 \\
\hline $\mathrm{N}$ & 0.927633 & -0.621077 & -1.151460 \\
\hline $\mathrm{N}$ & -0.370719 & -0.261908 & -2.865215 \\
\hline $\mathrm{C}$ & -1.427558 & -0.379853 & -3.876917 \\
\hline $\mathrm{H}$ & -0.972656 & -0.161710 & -4.849289 \\
\hline $\mathrm{H}$ & -1.741051 & -1.423595 & -3.895568 \\
\hline $\mathrm{C}$ & -2.619163 & 0.548137 & -3.618361 \\
\hline $\mathrm{H}$ & -3.046323 & 0.330198 & -2.634151 \\
\hline $\mathrm{H}$ & -2.271178 & 1.586905 & -3.578603 \\
\hline $\mathrm{C}$ & -3.690409 & 0.395545 & -4.700619 \\
\hline $\mathrm{H}$ & -4.074700 & -0.629581 & -4.737140 \\
\hline $\mathrm{H}$ & -4.537436 & 1.058475 & -4.501578 \\
\hline $\mathrm{H}$ & -3.299797 & 0.643198 & -5.695067 \\
\hline $\mathrm{H}$ & 0.627264 & -4.260266 & 1.238557 \\
\hline $\mathrm{Mn}$ & -1.013173 & -2.761619 & -1.037187 \\
\hline $\mathrm{C}$ & -1.582548 & -4.279966 & -0.170995 \\
\hline $\mathrm{O}$ & -1.936146 & -5.295564 & 0.256306 \\
\hline $\mathrm{C}$ & -0.170948 & -3.769882 & -2.270352 \\
\hline $\mathrm{O}$ & 0.363433 & -4.405580 & -3.084948 \\
\hline $\mathrm{C}$ & -2.590270 & -2.715819 & -1.899866 \\
\hline $\mathrm{O}$ & -3.640220 & -2.697753 & -2.392317 \\
\hline $\mathrm{C}$ & -3.648326 & -0.688633 & 1.821358 \\
\hline $\mathrm{C}$ & -4.312535 & -0.790039 & 3.037795 \\
\hline $\mathrm{C}$ & -3.992718 & -1.782089 & 3.980433 \\
\hline $\mathrm{C}$ & -2.956673 & -2.660136 & 3.636948 \\
\hline $\mathrm{C}$ & -2.271863 & -2.566885 & 2.426717 \\
\hline $\mathrm{C}$ & -2.591940 & -1.575546 & 1.464313 \\
\hline $\mathrm{H}$ & -3.924657 & 0.094521 & 1.117536 \\
\hline $\mathrm{H}$ & -5.110310 & -0.081975 & 3.259386 \\
\hline $\mathrm{H}$ & -2.668162 & -3.439924 & 4.341072 \\
\hline $\mathrm{H}$ & -1.454910 & -3.252178 & 2.230300 \\
\hline $\mathrm{C}$ & -4.747368 & -1.907105 & 5.283023 \\
\hline $\mathrm{H}$ & -4.897072 & -0.933073 & 5.765198 \\
\hline $\mathrm{H}$ & -5.745459 & -2.346982 & 5.145650 \\
\hline $\mathrm{H}$ & -4.212270 & -2.545736 & 5.994272 \\
\hline $\mathrm{N}$ & -1.897303 & -1.417053 & 0.288394 \\
\hline $\mathrm{H}$ & -2.304193 & -0.638128 & -0.220563 \\
\hline $\mathrm{H}$ & -3.483976 & 2.626225 & 0.089638 \\
\hline I & 6.479294 & 0.735159 & 0.505375 \\
\hline $\mathrm{C}$ & -5.024876 & 4.640556 & -0.349993 \\
\hline $\mathrm{C}$ & -5.303185 & 3.135003 & -0.486301 \\
\hline $\mathrm{H}$ & -4.407120 & 4.841407 & 0.532258 \\
\hline $\mathrm{H}$ & -4.495406 & 5.015711 & -1.232445 \\
\hline $\mathrm{H}$ & -5.954121 & 5.211266 & -0.243046 \\
\hline $\mathrm{C}$ & -6.124967 & 2.850185 & -1.745988 \\
\hline
\end{tabular}




$\begin{array}{lrrr}\mathrm{C} & -6.027027 & 2.608667 & 0.762661 \\ \mathrm{H} & -6.286799 & 1.773718 & -1.861351 \\ \mathrm{H} & -7.102301 & 3.341503 & -1.697829 \\ \mathrm{H} & -5.601765 & 3.214839 & -2.636271 \\ \mathrm{H} & -6.980794 & 3.124993 & 0.921063 \\ \mathrm{H} & -6.230959 & 1.537732 & 0.665451 \\ \mathrm{H} & -5.413174 & 2.758863 & 1.657979 \\ \mathrm{O} & -4.065131 & 2.425782 & -0.665687 \\ \mathrm{C} & 1.427856 & 3.026133 & 2.356693 \\ \mathrm{C} & 2.260576 & 2.539243 & 3.363023 \\ \mathrm{C} & 1.752915 & 1.664473 & 4.328453 \\ \mathrm{C} & 0.410401 & 1.278116 & 4.290615 \\ \mathrm{C} & -0.429759 & 1.770408 & 3.294261 \\ \mathrm{C} & 0.077242 & 2.645508 & 2.322358 \\ \mathrm{H} & 1.814972 & 3.704502 & 1.604783 \\ \mathrm{H} & 3.302899 & 2.842795 & 3.395818 \\ \mathrm{H} & 2.404844 & 1.285750 & 5.110881 \\ \mathrm{H} & 0.016883 & 0.592945 & 5.035269 \\ \mathrm{H} & -1.472303 & 1.475063 & 3.248364 \\ \mathrm{C} & -0.857510 & 3.156544 & 1.285344 \\ \mathrm{O} & -2.072689 & 3.025199 & 1.341070 \\ \mathrm{O} & -0.228019 & 3.783625 & 0.277767 \\ \mathrm{C} & -1.060843 & 4.363909 & -0.744031 \\ \mathrm{H} & -0.380971 & 4.637488 & -1.551194 \\ \mathrm{H} & -1.808907 & 3.651996 & -1.097356 \\ \mathrm{H} & -1.561570 & 5.257256 & -0.359439 \\ \mathrm{Na} & 7.050701 & -1.216126 & -1.464643 \\ & & & \end{array}$

7'-ts

Zero-point correction $=0.719949$

Thermal correction to Energy= 0.773101

Thermal correction to Enthalpy $=0.774045$

Thermal correction to Gibbs Free Energy= 0.620813

Sum of electronic and zero-point Energies $=-2382.536985$

Sum of electronic and thermal Energies $=-2382.483833$

Sum of electronic and thermal Enthalpies $=-2382.482889$

Sum of electronic and thermal Free Energies $=-2382.636121$

Cartesian coordinates

$\begin{array}{lrrr}\mathrm{C} & 1.287825 & -3.033563 & 0.467167 \\ \mathrm{C} & 2.447533 & -3.296665 & 1.183557 \\ \mathrm{C} & 3.526357 & -2.428022 & 1.032596 \\ \mathrm{C} & 3.406551 & -1.325359 & 0.193744 \\ \mathrm{C} & 2.191992 & -1.117665 & -0.463094 \\ \mathrm{~N} & 1.148828 & -1.966941 & -0.341828 \\ \mathrm{H} & 4.462792 & -2.598863 & 1.553455 \\ \mathrm{H} & 2.497429 & -4.165688 & 1.830682 \\ \mathrm{H} & 4.248599 & -0.668894 & 0.052140 \\ \mathrm{C} & 2.678737 & 1.137956 & -1.574446 \\ \mathrm{C} & 0.717400 & -0.001302 & -1.984603 \\ \mathrm{C} & 1.887193 & 1.882244 & -2.464694 \\ \mathrm{C} & 3.910673 & 1.634625 & -1.140243 \\ \mathrm{C} & 2.294267 & 3.115935 & -2.967681 \\ \mathrm{C} & 4.318158 & 2.870215 & -1.643843 \\ \mathrm{H} & 4.549651 & 1.122023 & -0.435766 \\ \mathrm{C} & 3.530742 & 3.599071 & -2.545868 \\ \mathrm{H} & 1.671717 & 3.681402 & -3.652137 \\ \mathrm{H} & 5.273187 & 3.272044 & -1.318461 \\ \mathrm{H} & 3.883402 & 4.556975 & -2.915037 \\ \mathrm{~N} & 1.924901 & -0.023924 & -1.307156 \\ \mathrm{~N} & 0.712699 & 1.151082 & -2.684067 \\ \mathrm{C} & -0.395296 & 1.668806 & -3.496643 \\ \mathrm{H} & 0.043544 & 2.139208 & -4.381594 \\ \mathrm{H} & -0.977772 & 0.818015 & -3.844533\end{array}$




\begin{tabular}{|c|c|c|c|}
\hline $\mathrm{C}$ & -1.274425 & 2.666578 & -2.729828 \\
\hline $\mathrm{H}$ & -1.666595 & 2.189122 & -1.825873 \\
\hline $\mathrm{H}$ & -0.655046 & 3.502138 & -2.387430 \\
\hline $\mathrm{C}$ & -2.421748 & 3.191163 & -3.597010 \\
\hline $\mathrm{H}$ & -3.078901 & 2.378114 & -3.928469 \\
\hline $\mathrm{H}$ & -3.034412 & 3.900978 & -3.034771 \\
\hline $\mathrm{H}$ & -2.049778 & 3.704596 & -4.491290 \\
\hline $\mathrm{H}$ & 0.433256 & -3.696009 & 0.535046 \\
\hline $\mathrm{Mn}$ & -0.477515 & -1.602641 & -1.623903 \\
\hline $\mathrm{C}$ & -1.490056 & -3.017552 & -0.986661 \\
\hline $\mathrm{O}$ & -2.120353 & -3.921415 & -0.649837 \\
\hline $\mathrm{C}$ & 0.317950 & -2.606431 & -2.862782 \\
\hline $\mathrm{O}$ & 0.848400 & -3.256233 & -3.662132 \\
\hline $\mathrm{C}$ & -1.823827 & -1.223374 & -2.781451 \\
\hline $\mathrm{O}$ & -2.672865 & -1.065669 & -3.552833 \\
\hline $\mathrm{C}$ & -5.107983 & -0.293466 & -1.082497 \\
\hline $\mathrm{C}$ & -6.426732 & -0.737890 & -1.174550 \\
\hline $\mathrm{C}$ & -6.877226 & -1.836382 & -0.434077 \\
\hline $\mathrm{C}$ & -5.945956 & -2.483074 & 0.391446 \\
\hline $\mathrm{C}$ & -4.623049 & -2.058485 & 0.480742 \\
\hline $\mathrm{C}$ & -4.178511 & -0.943456 & -0.252570 \\
\hline $\mathrm{H}$ & -4.791625 & 0.572293 & -1.658537 \\
\hline $\mathrm{H}$ & -7.117356 & -0.210761 & -1.829996 \\
\hline $\mathrm{H}$ & -6.259268 & -3.347867 & 0.973629 \\
\hline $\mathrm{H}$ & -3.930799 & -2.593763 & 1.118846 \\
\hline $\mathrm{C}$ & -8.312727 & -2.297524 & -0.502603 \\
\hline $\mathrm{H}$ & -8.387049 & -3.390580 & -0.526771 \\
\hline $\mathrm{H}$ & -8.887591 & -1.957865 & 0.369153 \\
\hline $\mathrm{H}$ & -8.816806 & -1.909961 & -1.394110 \\
\hline $\mathrm{N}$ & -2.823573 & -0.510787 & -0.225134 \\
\hline $\mathrm{H}$ & -2.749677 & 0.254219 & -0.891745 \\
\hline $\mathrm{H}$ & -3.056568 & 2.596018 & 2.212669 \\
\hline I & 7.304698 & -0.091968 & 1.200088 \\
\hline $\mathrm{C}$ & -5.206124 & 3.699146 & 1.292410 \\
\hline $\mathrm{C}$ & -4.035228 & 4.302508 & 2.089443 \\
\hline $\mathrm{H}$ & -4.837152 & 2.901735 & 0.641059 \\
\hline $\mathrm{H}$ & -5.942058 & 3.259780 & 1.974446 \\
\hline $\mathrm{H}$ & -5.715368 & 4.450664 & 0.677165 \\
\hline $\mathrm{C}$ & -4.549153 & 5.324560 & 3.109949 \\
\hline $\mathrm{C}$ & -3.025835 & 4.962046 & 1.131243 \\
\hline $\mathrm{H}$ & -3.715747 & 5.742979 & 3.685450 \\
\hline $\mathrm{H}$ & -5.081098 & 6.149517 & 2.623152 \\
\hline $\mathrm{H}$ & -5.232764 & 4.842370 & 3.817256 \\
\hline $\mathrm{H}$ & -3.478959 & 5.776721 & 0.551773 \\
\hline $\mathrm{H}$ & -2.181170 & 5.371946 & 1.695916 \\
\hline $\mathrm{H}$ & -2.633166 & 4.215772 & 0.432842 \\
\hline $\mathrm{O}$ & -3.385540 & 3.286282 & 2.845863 \\
\hline $\mathrm{C}$ & -1.843944 & -1.729338 & 2.644787 \\
\hline $\mathrm{C}$ & -2.120548 & -2.427392 & 3.821466 \\
\hline $\mathrm{C}$ & -3.110240 & -1.966518 & 4.693215 \\
\hline $\mathrm{C}$ & -3.809427 & -0.798448 & 4.380816 \\
\hline $\mathrm{C}$ & -3.529357 & -0.101881 & 3.203548 \\
\hline $\mathrm{C}$ & -2.550311 & -0.562986 & 2.315556 \\
\hline $\mathrm{H}$ & -1.064342 & -2.077234 & 1.975069 \\
\hline $\mathrm{H}$ & -1.559393 & -3.328398 & 4.061958 \\
\hline $\mathrm{H}$ & -3.327977 & -2.507022 & 5.610537 \\
\hline $\mathrm{H}$ & -4.573543 & -0.423923 & 5.057310 \\
\hline $\mathrm{H}$ & -4.049950 & 0.821960 & 2.973924 \\
\hline $\mathrm{C}$ & -2.270033 & 0.229176 & 1.023751 \\
\hline $\mathrm{O}$ & -2.641362 & 1.468263 & 1.015434 \\
\hline $\mathrm{O}$ & -0.800390 & 0.045447 & 0.710573 \\
\hline $\mathrm{C}$ & 0.013375 & 0.893020 & 1.504998 \\
\hline $\mathrm{H}$ & 0.985741 & 0.996187 & 1.017174 \\
\hline
\end{tabular}




$\begin{array}{lrrr}\mathrm{H} & -0.442026 & 1.883111 & 1.599186 \\ \mathrm{H} & 0.173121 & 0.479521 & 2.513061 \\ \mathrm{Na} & 9.971925 & 0.354244 & 2.050507\end{array}$

4"

Zero-point correction $=0.458598$

Thermal correction to Energy $=0.498313$

Thermal correction to Enthalpy $=0.499257$

Thermal correction to Gibbs Free Energy= 0.376982

Sum of electronic and zero-point Energies= -1704.300605

Sum of electronic and thermal Energies $=-1704.260890$

Sum of electronic and thermal Enthalpies $=-1704.259946$

Sum of electronic and thermal Free Energies $=-1704.382221$

Cartesian coordinates

\begin{tabular}{|c|c|c|c|}
\hline $\mathrm{Mn}$ & 2.263183 & 0.708459 & -1.938615 \\
\hline $\mathrm{C}$ & 3.417017 & 2.194781 & -1.948972 \\
\hline $\mathrm{O}$ & 4.169027 & 3.054901 & -2.041357 \\
\hline $\mathrm{C}$ & 2.351708 & 0.620358 & -3.767076 \\
\hline $\mathrm{O}$ & 2.410699 & 0.556736 & -4.916733 \\
\hline $\mathrm{C}$ & 3.772705 & -0.368756 & -1.631438 \\
\hline $\mathrm{O}$ & 4.717557 & -0.977012 & -1.407168 \\
\hline $\mathrm{C}$ & 2.488295 & 1.241189 & 2.449443 \\
\hline $\mathrm{C}$ & 2.565344 & 2.191949 & 3.457380 \\
\hline $\mathrm{C}$ & 2.441582 & 3.568895 & 3.201370 \\
\hline $\mathrm{C}$ & 2.227992 & 3.937522 & 1.869613 \\
\hline $\mathrm{C}$ & 2.143414 & 2.997109 & 0.840824 \\
\hline $\mathrm{C}$ & 2.274225 & 1.607100 & 1.087914 \\
\hline $\mathrm{H}$ & 2.596439 & 0.188467 & 2.701741 \\
\hline $\mathrm{H}$ & 2.730219 & 1.854688 & 4.479935 \\
\hline $\mathrm{H}$ & 2.117563 & 4.991626 & 1.619215 \\
\hline $\mathrm{H}$ & 1.945946 & 3.351343 & -0.165455 \\
\hline $\mathrm{C}$ & 2.555347 & 4.586623 & 4.311011 \\
\hline $\mathrm{H}$ & 1.955295 & 4.305088 & 5.185208 \\
\hline $\mathrm{H}$ & 3.590572 & 4.701080 & 4.662703 \\
\hline $\mathrm{H}$ & 2.214883 & 5.574725 & 3.984130 \\
\hline $\mathrm{N}$ & 2.173976 & 0.642628 & 0.120872 \\
\hline $\mathrm{H}$ & 2.260784 & -0.295974 & 0.499784 \\
\hline $\mathrm{H}$ & 1.141480 & -2.528954 & 0.766836 \\
\hline $\mathrm{C}$ & 2.459995 & -4.774152 & 0.513985 \\
\hline $\mathrm{C}$ & 2.723475 & -3.530494 & 1.378182 \\
\hline $\mathrm{H}$ & 1.381946 & -4.955039 & 0.428450 \\
\hline $\mathrm{H}$ & 2.860580 & -4.632605 & -0.495353 \\
\hline $\mathrm{H}$ & 2.920858 & -5.670899 & 0.943853 \\
\hline $\mathrm{C}$ & 4.219056 & -3.221534 & 1.452755 \\
\hline $\mathrm{C}$ & 2.132908 & -3.718986 & 2.784207 \\
\hline $\mathrm{H}$ & 4.398083 & -2.321056 & 2.048342 \\
\hline $\mathrm{H}$ & 4.762638 & -4.051670 & 1.916262 \\
\hline $\mathrm{H}$ & 4.634597 & -3.058285 & 0.454053 \\
\hline $\mathrm{H}$ & 2.586808 & -4.576958 & 3.292909 \\
\hline $\mathrm{H}$ & 2.302961 & -2.829157 & 3.399464 \\
\hline $\mathrm{H}$ & 1.052743 & -3.892922 & 2.729286 \\
\hline $\mathrm{O}$ & 2.105585 & -2.389340 & 0.745394 \\
\hline $\mathrm{C}$ & -3.780547 & -0.961499 & 0.352614 \\
\hline $\mathrm{C}$ & -4.256367 & 0.348115 & 0.401131 \\
\hline $\mathrm{C}$ & -3.377491 & 1.390882 & 0.712314 \\
\hline $\mathrm{C}$ & -2.028660 & 1.130461 & 0.978810 \\
\hline $\mathrm{C}$ & -1.552575 & -0.178118 & 0.932739 \\
\hline $\mathrm{C}$ & -2.428570 & -1.231456 & 0.619274 \\
\hline $\mathrm{H}$ & -4.455542 & -1.775235 & 0.111946 \\
\hline $\mathrm{H}$ & -5.307151 & 0.556581 & 0.203958 \\
\hline $\mathrm{H}$ & -3.751561 & 2.410428 & 0.748307 \\
\hline $\mathrm{H}$ & -1.348735 & 1.941429 & 1.222022 \\
\hline $\mathrm{H}$ & -0.509976 & -0.393984 & 1.136609 \\
\hline
\end{tabular}




$\begin{array}{lrrr}\mathrm{C} & -1.882610 & -2.609489 & 0.582753 \\ \mathrm{O} & -0.734884 & -2.919113 & 0.889163 \\ \mathrm{O} & -2.783879 & -3.525652 & 0.190952 \\ \mathrm{C} & -2.321305 & -4.883171 & 0.094123 \\ \mathrm{H} & -3.203433 & -5.474531 & -0.152227 \\ \mathrm{H} & -1.572397 & -4.977799 & -0.697032 \\ \mathrm{H} & -1.890383 & -5.219744 & 1.040525 \\ \mathrm{Br} & -8.170844 & 1.124806 & -0.333333 \\ \mathrm{C} & 1.114494 & -0.792733 & -1.913255 \\ \mathrm{O} & 0.396407 & -1.682276 & -1.959028 \\ \mathrm{C} & 0.741871 & 1.831805 & -1.935910 \\ \mathrm{O} & -0.185624 & 2.499613 & -1.966508\end{array}$

7"-ts

Zero-point correction $=0.458849$

Thermal correction to Energy $=0.496606$

Thermal correction to Enthalpy $=0.497550$

Thermal correction to Gibbs Free Energy= 0.383090

Sum of electronic and zero-point Energies $=-1704.230133$

Sum of electronic and thermal Energies $=-1704.192375$

Sum of electronic and thermal Enthalpies $=-1704.191431$

Sum of electronic and thermal Free Energies= -1704.305891

Cartesian coordinates

$\begin{array}{lrrr}\mathrm{Mn} & 1.509828 & -1.380552 & -0.839145 \\ \mathrm{C} & 0.741124 & -2.709525 & 0.254450 \\ \mathrm{O} & 0.300438 & -3.535765 & 0.911226 \\ \mathrm{C} & 2.882951 & -2.450131 & -1.360135 \\ \mathrm{O} & 3.734398 & -3.144264 & -1.695026 \\ \mathrm{C} & 0.443568 & -1.981437 & -2.270032 \\ \mathrm{O} & -0.177065 & -2.376599 & -3.147887 \\ \mathrm{C} & -3.273888 & -1.626890 & -1.305670 \\ \mathrm{C} & -4.392648 & -2.446682 & -1.172341 \\ \mathrm{C} & -4.660930 & -3.120735 & 0.025418 \\ \mathrm{C} & -3.753653 & -2.949468 & 1.081200 \\ \mathrm{C} & -2.627281 & -2.140551 & 0.958646 \\ \mathrm{C} & -2.371196 & -1.453755 & -0.241358 \\ \mathrm{H} & -3.099395 & -1.105680 & -2.243896 \\ \mathrm{H} & -5.072250 & -2.554978 & -2.014243 \\ \mathrm{H} & -3.927420 & -3.465685 & 2.022567 \\ \mathrm{H} & -1.950707 & -2.038852 & 1.796957 \\ \mathrm{C} & -5.894908 & -3.974284 & 0.190091 \\ \mathrm{H} & -5.692329 & -4.861790 & 0.799191 \\ \mathrm{H} & -6.699173 & -3.418815 & 0.690183 \\ \mathrm{H} & -6.284108 & -4.310779 & -0.775922 \\ \mathrm{~N} & -1.212169 & -0.649716 & -0.430525 \\ \mathrm{H} & -1.243874 & -0.309791 & -1.390484 \\ \mathrm{H} & -2.564529 & 3.094260 & -0.148498 \\ \mathrm{C} & -4.823465 & 2.693587 & -1.415410 \\ \mathrm{C} & -3.927520 & 3.938547 & -1.286573 \\ \mathrm{H} & -4.207448 & 1.789220 & -1.408246 \\ \mathrm{H} & -5.517021 & 2.635093 & -0.569011 \\ \mathrm{H} & -5.412818 & 2.705390 & -2.340588 \\ \mathrm{C} & -4.782197 & 5.205215 & -1.170720 \\ \mathrm{C} & -2.977091 & 4.037256 & -2.493138 \\ \mathrm{H} & -4.143230 & 6.087237 & -1.055728 \\ \mathrm{H} & -5.411027 & 5.347226 & -2.056793 \\ \mathrm{H} & -5.433513 & 5.145830 & -0.291963 \\ \mathrm{H} & -3.524765 & 4.124601 & -3.439542 \\ \mathrm{H} & -2.325892 & 4.912119 & -2.391516 \\ \mathrm{C} & -2.340949 & 3.147557 & -2.542746 \\ \mathrm{H} & -3.170849 & 3.868883 & -0.079501 \\ \mathrm{H} & -0.924255 & -0.013294 & 4.061167\end{array}$




$\begin{array}{lrrr}\mathrm{C} & -2.091386 & 0.615509 & 4.501528 \\ \mathrm{C} & -2.904945 & 1.277455 & 3.580661 \\ \mathrm{C} & -2.560562 & 1.307297 & 2.227624 \\ \mathrm{C} & -1.399049 & 0.671522 & 1.775379 \\ \mathrm{H} & 0.328785 & -0.465580 & 2.375236 \\ \mathrm{H} & -0.275090 & -0.519613 & 4.770808 \\ \mathrm{H} & -2.357646 & 0.597453 & 5.555022 \\ \mathrm{H} & -3.808733 & 1.779859 & 3.915092 \\ \mathrm{H} & -3.176193 & 1.845023 & 1.515362 \\ \mathrm{C} & -1.064773 & 0.737833 & 0.276909 \\ \mathrm{O} & -1.646071 & 1.645779 & -0.412542 \\ \mathrm{O} & 0.473493 & 0.812063 & 0.170057 \\ \mathrm{C} & 0.981700 & 2.108828 & 0.485579 \\ \mathrm{H} & 1.994532 & 2.195161 & 0.080957 \\ \mathrm{H} & 0.349208 & 2.875563 & 0.027779 \\ \mathrm{H} & 1.024557 & 2.269578 & 1.570376 \\ \mathrm{C} & 2.509523 & -0.729295 & 0.648251 \\ \mathrm{O} & 3.140584 & -0.381617 & 1.532379 \\ \mathrm{C} & 2.083175 & 0.097149 & -1.890894 \\ \mathrm{O} & 2.404163 & 0.974970 & -2.545905 \\ \mathrm{Br} & 8.093198 & 1.314010 & 0.419065\end{array}$

4n"

Zero-point correction $=0.459410$

Thermal correction to Energy $=0.498771$

Thermal correction to Enthalpy= 0.499715

Thermal correction to Gibbs Free Energy $=0.379760$

Sum of electronic and zero-point Energies $=-1690.772144$

Sum of electronic and thermal Energies $=-1690.732784$

Sum of electronic and thermal Enthalpies $=-1690.731839$

Sum of electronic and thermal Free Energies= -1690.851794

Cartesian coordinates

$\begin{array}{lrrr}\mathrm{Mn} & 1.689681 & -1.292692 & -1.419499 \\ \mathrm{C} & 3.539230 & -1.006014 & -1.228903 \\ \mathrm{O} & 4.677981 & -0.917938 & -1.143227 \\ \mathrm{C} & 1.997622 & -2.484050 & -2.780846 \\ \mathrm{O} & 2.182814 & -3.253242 & -3.619095 \\ \mathrm{C} & 1.810146 & -2.568760 & -0.045736 \\ \mathrm{O} & 1.921015 & -3.317983 & 0.813396 \\ \mathrm{C} & 1.894318 & 1.143993 & 2.197779 \\ \mathrm{C} & 2.632207 & 2.130701 & 2.838826 \\ \mathrm{C} & 3.548128 & 2.941173 & 2.146320 \\ \mathrm{C} & 3.674386 & 2.712531 & 0.772174 \\ \mathrm{C} & 2.934854 & 1.731725 & 0.110709 \\ \mathrm{C} & 2.013940 & 0.907048 & 0.800460 \\ \mathrm{H} & 1.195740 & 0.537787 & 2.769550 \\ \mathrm{H} & 2.501015 & 2.270112 & 3.910619 \\ \mathrm{H} & 4.367286 & 3.321134 & 0.193676 \\ \mathrm{H} & 3.059478 & 1.623984 & -0.962347 \\ \mathrm{C} & 4.362910 & 3.992123 & 2.861315 \\ \mathrm{H} & 3.733036 & 4.663257 & 3.457345 \\ \mathrm{H} & 5.093383 & 3.548667 & 3.551271 \\ \mathrm{H} & 4.923089 & 4.612344 & 2.153453 \\ \mathrm{~N} & 1.212143 & -0.023904 & 0.171149 \\ \mathrm{H} & 0.601941 & -0.489850 & 0.839681 \\ \mathrm{H} & -1.845389 & -1.146138 & 1.139149 \\ \mathrm{C} & -2.687915 & -3.193961 & 2.486052 \\ \mathrm{C} & -1.598387 & -2.160732 & 2.809439 \\ \mathrm{H} & -3.521086 & -2.722791 & 1.953926 \\ \mathrm{H} & -2.284377 & -3.988224 & 1.849290 \\ \mathrm{H} & -3.084953 & -3.655355 & 3.398227 \\ \mathrm{C} & -0.400562 & -2.828996 & 3.484010 \\ \mathrm{C} & -2.162684 & -1.034196 & 3.689045\end{array}$




$\begin{array}{lrrr}\mathrm{H} & 0.398717 & -2.103727 & 3.663796 \\ \mathrm{H} & -0.690976 & -3.262719 & 4.446055 \\ \mathrm{H} & 0.001415 & -3.629767 & 2.856270 \\ \mathrm{H} & -2.531234 & -1.422358 & 4.644464 \\ \mathrm{H} & -1.390541 & -0.286000 & 3.900165 \\ \mathrm{H} & -2.994267 & -0.529560 & 3.187023 \\ \mathrm{O} & -1.101147 & -1.599960 & 1.575210 \\ \mathrm{C} & -4.068727 & 2.766855 & -1.380205 \\ \mathrm{C} & -3.442454 & 3.955284 & -1.747713 \\ \mathrm{C} & -2.101783 & 4.172196 & -1.420174 \\ \mathrm{C} & -1.382234 & 3.197594 & -0.723746 \\ \mathrm{C} & -2.003446 & 2.006349 & -0.351579 \\ \mathrm{C} & -3.350238 & 1.785467 & -0.678435 \\ \mathrm{H} & -5.108700 & 2.594911 & -1.631418 \\ \mathrm{H} & -4.000971 & 4.713589 & -2.288603 \\ \mathrm{H} & -1.615867 & 5.100597 & -1.707982 \\ \mathrm{H} & -0.341164 & 3.364078 & -0.466761 \\ \mathrm{H} & -1.446958 & 1.246831 & 0.186689 \\ \mathrm{C} & -3.980941 & 0.505898 & -0.262674 \\ \mathrm{O} & -3.419485 & -0.379141 & 0.370401 \\ \mathrm{O} & -5.262598 & 0.401923 & -0.650925 \\ \mathrm{C} & -5.939814 & -0.815413 & -0.286061 \\ \mathrm{H} & -6.939391 & -0.732930 & -0.714393 \\ \mathrm{H} & -5.419831 & -1.684956 & -0.695978 \\ \mathrm{H} & -6.002027 & -0.910052 & 0.801127 \\ \mathrm{C} & -0.172003 & -1.556490 & -1.568588 \\ \mathrm{O} & -1.295790 & -1.710577 & -1.717063 \\ \mathrm{C} & 1.524488 & 0.193353 & -2.563048 \\ \mathrm{O} & 1.393379 & 1.088130 & -3.265622\end{array}$

7n"-ts

Zero-point correction $=0.459539$

Thermal correction to Energy= 0.496894

Thermal correction to Enthalpy $=0.497838$

Thermal correction to Gibbs Free Energy $=0.387177$

Sum of electronic and zero-point Energies $=-1690.701648$

Sum of electronic and thermal Energies $=-1690.664293$

Sum of electronic and thermal Enthalpies $=-1690.663349$

Sum of electronic and thermal Free Energies $=-1690.774010$

Cartesian coordinates

$\begin{array}{lrrr}\mathrm{Mn} & -2.833930 & -0.629139 & -0.422756 \\ \mathrm{C} & -2.896520 & 1.050604 & 0.442682 \\ \mathrm{O} & -2.965817 & 2.075724 & 0.943162 \\ \mathrm{C} & -4.607784 & -0.626134 & -0.795393 \\ \mathrm{O} & -5.733205 & -0.623489 & -1.030513 \\ \mathrm{C} & -2.443527 & 0.278805 & -2.030726 \\ \mathrm{O} & -2.254453 & 0.836190 & -3.012320 \\ \mathrm{C} & 0.782791 & 2.209202 & -1.850434 \\ \mathrm{C} & 1.199518 & 3.526773 & -2.027654 \\ \mathrm{C} & 1.160459 & 4.448529 & -0.974321 \\ \mathrm{C} & 0.675249 & 3.998561 & 0.262473 \\ \mathrm{C} & 0.243119 & 2.688498 & 0.450445 \\ \mathrm{C} & 0.298139 & 1.764716 & -0.607953 \\ \mathrm{H} & 0.838751 & 1.511165 & -2.682248 \\ \mathrm{H} & 1.568758 & 3.838674 & -3.001528 \\ \mathrm{H} & 0.625991 & 4.691645 & 1.099037 \\ \mathrm{H} & -0.130137 & 2.383479 & 1.419422 \\ \mathrm{C} & 1.640515 & 5.867999 & -1.152749 \\ \mathrm{H} & 1.013453 & 6.577130 & -0.601840 \\ \mathrm{H} & 2.666387 & 5.992085 & -0.782216 \\ \mathrm{H} & 1.637970 & 6.163705 & -2.206295 \\ \mathrm{~N} & -0.181131 & 0.427698 & -0.486722 \\ \mathrm{H} & -0.023447 & -0.020771 & -1.388978\end{array}$




$\begin{array}{lrrr}\mathrm{H} & 3.087534 & -1.835156 & 0.060339 \\ \mathrm{C} & 4.639616 & -0.585496 & -1.617429 \\ \mathrm{C} & 4.628347 & -2.048902 & -1.140762 \\ \mathrm{H} & 3.616375 & -0.200065 & -1.667401 \\ \mathrm{H} & 5.201912 & 0.038601 & -0.913948 \\ \mathrm{H} & 5.098784 & -0.480418 & -2.607953 \\ \mathrm{C} & 6.059045 & -2.567509 & -0.963778 \\ \mathrm{C} & 3.855354 & -2.928474 & -2.139589 \\ \mathrm{H} & 6.048952 & -3.605083 & -0.613200 \\ \mathrm{H} & 6.618006 & -2.526483 & -1.904998 \\ \mathrm{H} & 6.592242 & -1.966505 & -0.219566 \\ \mathrm{H} & 4.306491 & -2.905607 & -3.138812 \\ \mathrm{H} & 3.838716 & -3.968474 & -1.795304 \\ \mathrm{H} & 2.819544 & -2.582442 & -2.221863 \\ \mathrm{O} & 4.020648 & -2.134926 & 0.148560 \\ \mathrm{C} & -0.071560 & 0.120680 & 2.790638 \\ \mathrm{C} & 0.292948 & 0.587979 & 4.054427 \\ \mathrm{C} & 1.634726 & 0.844090 & 4.345743 \\ \mathrm{C} & 2.604905 & 0.619983 & 3.367830 \\ \mathrm{C} & 2.238629 & 0.150103 & 2.105152 \\ \mathrm{C} & 0.895293 & -0.096532 & 1.798570 \\ \mathrm{H} & -1.114069 & -0.087687 & 2.580829 \\ \mathrm{H} & -0.469867 & 0.744050 & 4.812859 \\ \mathrm{H} & 1.921201 & 1.205002 & 5.329814 \\ \mathrm{H} & 3.652969 & 0.802831 & 3.588589 \\ \mathrm{H} & 2.994849 & -0.056448 & 1.356644 \\ \mathrm{C} & 0.557753 & -0.612603 & 0.392664 \\ \mathrm{O} & 1.492620 & -1.174384 & -0.269413 \\ \mathrm{O} & -0.683819 & -1.570811 & 0.575467 \\ \mathrm{C} & -0.299975 & -2.865317 & 1.032480 \\ \mathrm{H} & -1.097663 & -3.578179 & 0.799101 \\ \mathrm{H} & 0.615062 & -3.179822 & 0.521697 \\ \mathrm{H} & -0.131966 & -2.872311 & 2.117384 \\ \mathrm{C} & -3.128738 & -1.523196 & 1.229059 \\ \mathrm{O} & -3.354941 & -2.066107 & 2.209104 \\ \mathrm{C} & -2.515868 & -2.285119 & -1.287242 \\ \mathrm{O} & -2.312940 & -3.273667 & -1.822598\end{array}$

